\title{
Characterization of the Molecular
}

\section{Chaperone Get3}

\section{Dissertation}

\author{
for the award of the degree \\ "Doctor of Philosophy" (Ph.D.) \\ Division of Mathematics and Natural Sciences \\ of the Georg-August-Univers ität Göttingen
}

\author{
submitted by \\ Wilhelm Voth \\ from Semipalatinsk, Kasachstan
}

Göttingen 2016 


\section{Thesis Committee}

Prof. Dr. Blanche Schwappach, Director of Biochemistry, Department of Molecular Biology, Universitätsmedizin Göttingen

Prof. Dr. Ursula Jakob, Department of Molecular, Cellular and Developmental Biology, University of Michigan

Prof. Dr. Wolfgang Wintermeyer, Department of Physical Biochemistry, Max Planck Institute for Biophysical Chemistry Göttingen

\section{Members of the Examination Board}

Referee: Prof. Dr. Blanche Schwappach (Reviewer), Director of Biochemistry, Department of Molecular Biology, Universitätsmedizin Göttingen

Second Referee: Prof. Dr. Ursula Jakob (Reviewer), Department of Molecular, Cellular and Developmental Biology, University of Michigan

\section{Further Members of the Examination Board}

Dr. Manfred Konrad, University Medical Center Göttingen, Max Planck Institute for Biophysical Chemistry Göttingen

Prof. Dr. Peter Rehling, Director of the Department of Biochemistry II, University Medical Center Göttingen

Prof. Dr. Jürgen Wienands, Department of Cellular and Molecular Immunology, University Medical Center Göttingen

Date of the oral examination: $20^{\text {th }}$ September 2016 


\section{Declaration}

I, Wilhelm Voth, hereby declare that this thesis was prepared by me independently, using only the references and resources stated here. Parts of this work have been published in a scientific journal.

Wilhelm Voth

Göttingen, 30 ${ }^{\text {th }}$ of June 2016 



\section{Abstract}

Proteins within the cellular environment are constantly challenged by environmental stress conditions that threaten their structure and function, and increase the risk of forming potentially toxic protein aggregates. Sudden exposure of cells to increased levels of reactive oxygen species (ROS) beyond the limits of the cellular detoxification machinery causes a rapid and significant drop in intracellular ATP-levels disturbing the cellular proteostasis network. This mostly ATP-dependent chaperone system becomes inactive under these conditions, making ROS-mediated protein unfolding and aggregation a potentially very challenging problem for organisms. Here we demonstrate that the yeast protein Get3, involved in ATP-dependent targeting of tail-anchored (TA) proteins under non-stress conditions, turns into an effective ATP-independent general chaperone under oxidative stress conditions. The process of Get3's activation as chaperone is reminiscent of Hsp33, the prokaryotic paradigm for a redoxregulated chaperone. It involves disulfide bond formation, zinc release and the conversion of Get3 into distinct, higher oligomeric structures which adopt an ATP-independent chaperone holdase function. Most importantly, the substantial structural rearrangements that expose binding sides for unfolded protein upon oxidation are fully reversible. Restoration of non-stress conditions return Get3 into an ATP-binding targeting factor for tail-anchored proteins. Unfolded substrates bound to chaperone active Get3 are then released and transferred to the ATP-dependent folding machinery for their refolding. Mutational studies revealed that Get3's chaperone activity is functionally distinct from and likely mutually exclusive with its targeting function. Indeed, in vivo complementation studies demonstrated that the oxidative stress sensitive phenotype that has long been observed for yeast cells lacking functional Get3 is specifically due to the absence of Get3's chaperone function. Our results provide convincing evidence that Get3 moonlights as a redox regulated chaperone in the proteostasis network of eukaryotic cells, effectively protecting proteins against oxidative damage and allowing their refolding upon return to non-stress conditions. 


\section{Contents}

1 INTRODUCTION

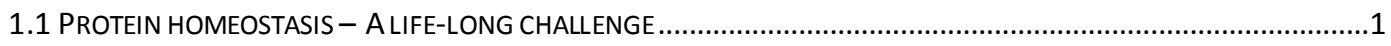

1.2 MOLECULAR CHAPERONES AND PROTEIN HOMEOSTASIS...........................................................................

1.2.1 Expression of molecular chaperones is controlled by the heat shock response .......................3

1.2.2 ATP-dependent chaperones ..................................................................................................

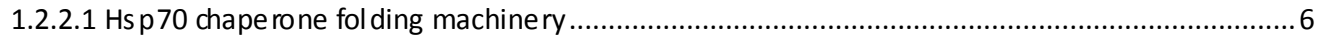

1.2.2.2 The Hsp60, Hsp90 and Hsp100 chape rone network............................................................ 8

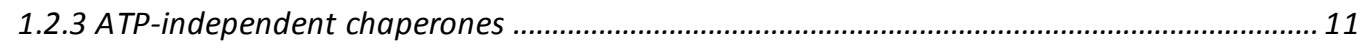

1.2.3.1 Small heat shock proteins fight protein aggrega tes ............................................................... 11

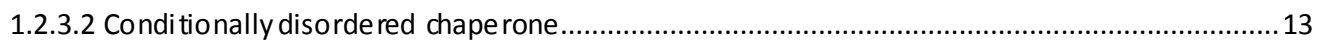

1.2.3.3 Oxida tive stress challenges the proteos tasis ne twork............................................................. 14

1.2.3.4 The redox-regulated chaperone holdase Hsp33.................................................................. 15

1.2.3.5 Peroxi redoxins exert a redox-dependent dual function ...........................................................19

1.3 THE GET PATHWAY IS INVOLVED IN THE BIOGENESIS OF TAIL-ANCHORED (TA)-PROTEINS ................................ 20

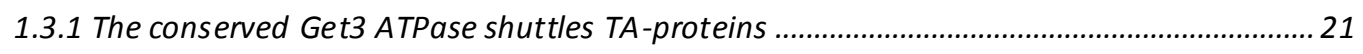

1.3.2 Get3 exhibits a general in vitro and in vivo chaperone function ...........................................25

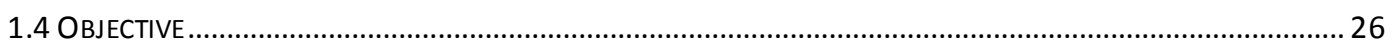

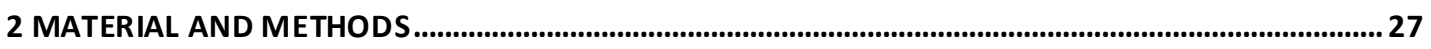

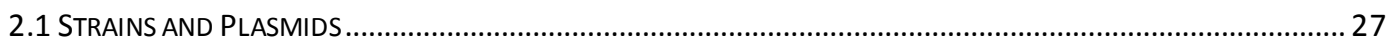

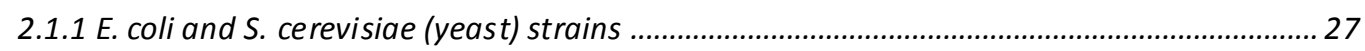

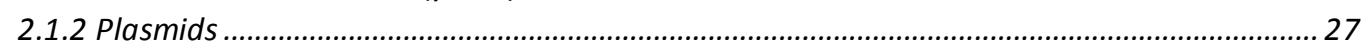

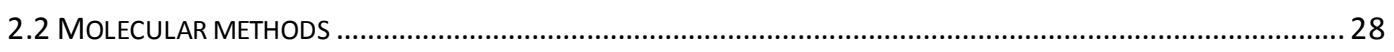

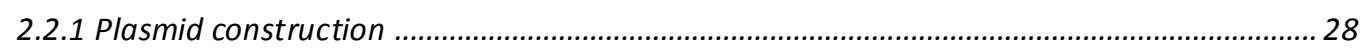

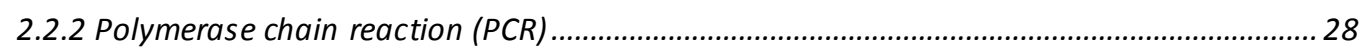

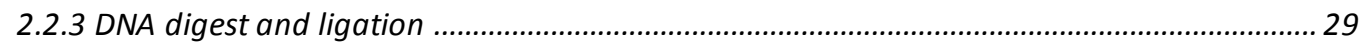

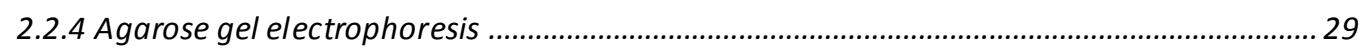

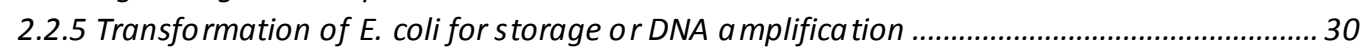

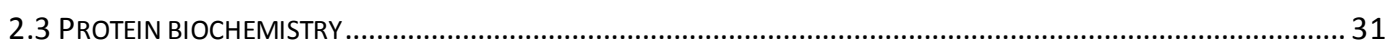

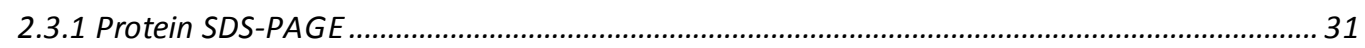

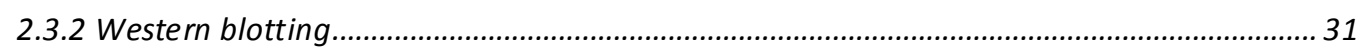

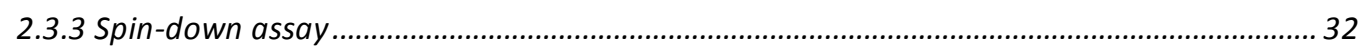

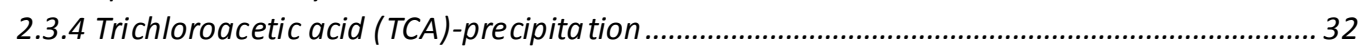

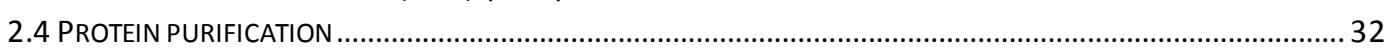

2.4.1 Purification of wild-type Get3 and Get3 mutant variants ..................................................... 32

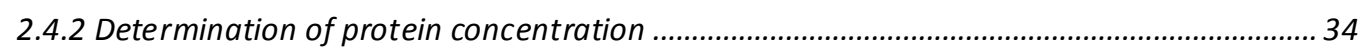

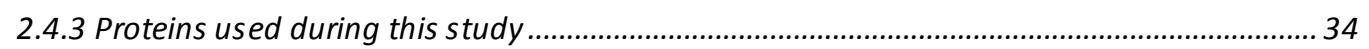

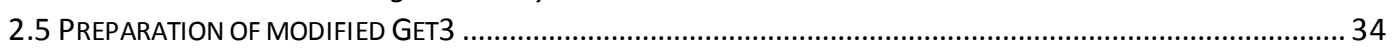

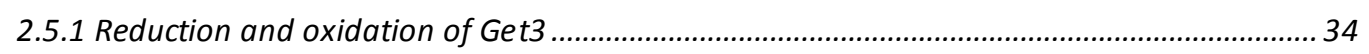

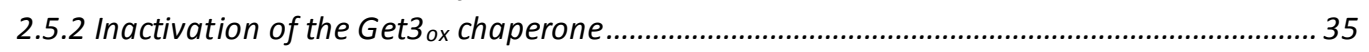

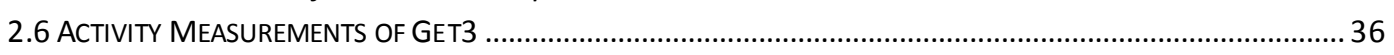

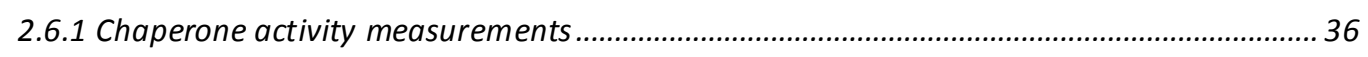

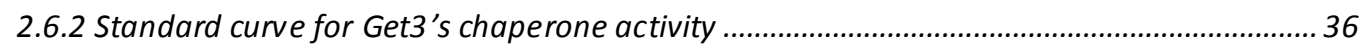

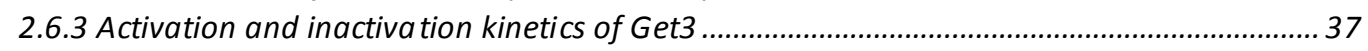

2.6.4 Chaperone-mediated reactivation of thermally unfolded luciferase .................................... 39

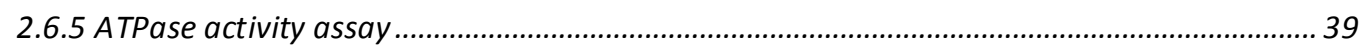

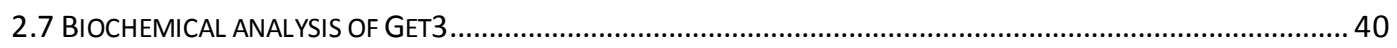




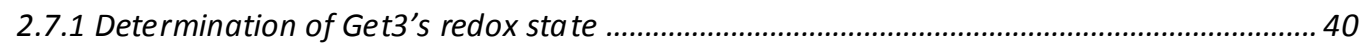

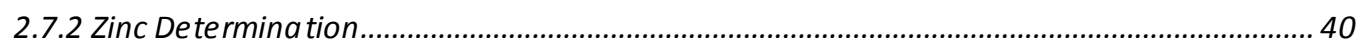

2.7.3 Analysis for metal content by inductively coupled plasma mass spectrometry (ICP) ......... 41

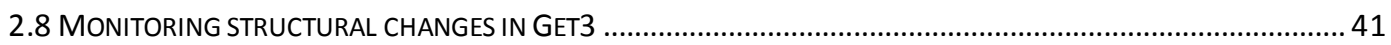

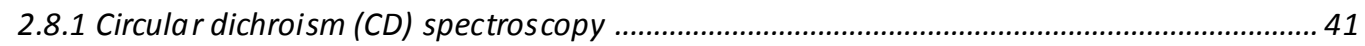

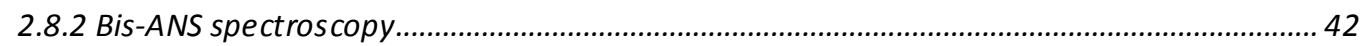

2.9 PURIFICATION OF VARIOUS GET3 SPECIES AND GET3-SUBSTRATE COMPLEX BY SIZE-EXCLUSION

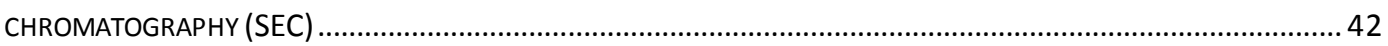

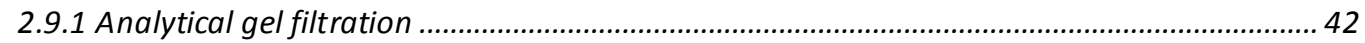

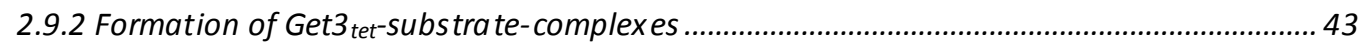

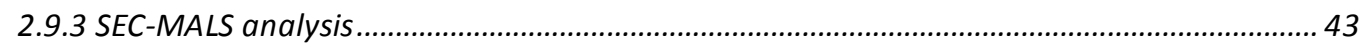

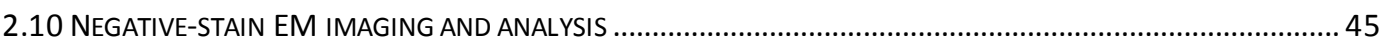

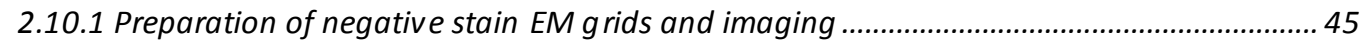

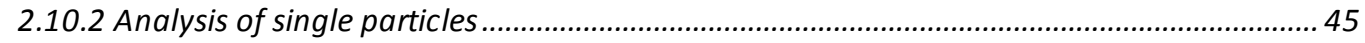

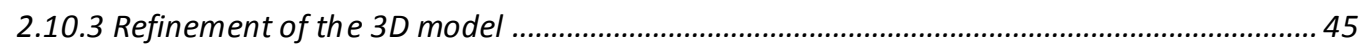

2.11 HYDROGEN/DEUTERIUM EXCHANGE EXPERIMENTS COMBINED WITH MASS SPECTROMETRY ............................ 46

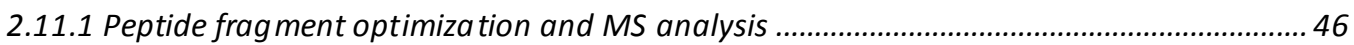

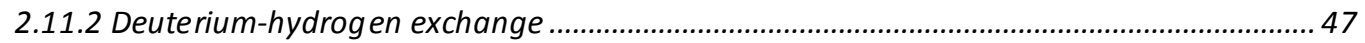

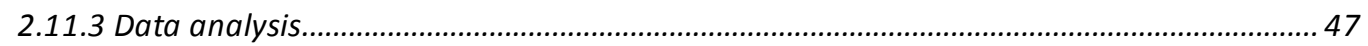

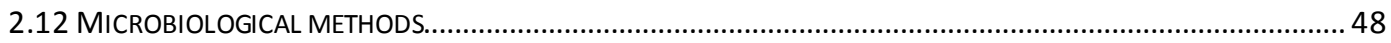

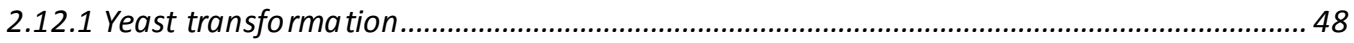

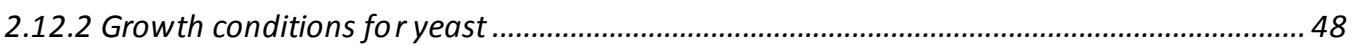

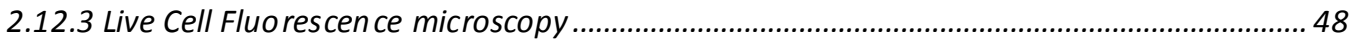

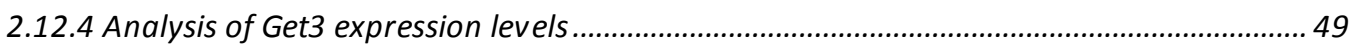

3 RESULTS

3.1 THE PROTEIN TARGETING FACTOR GET3 FUNCTIONS AS ATP-INDEPENDENT CHAPERONE UNDER OXIDATIVE STRESS

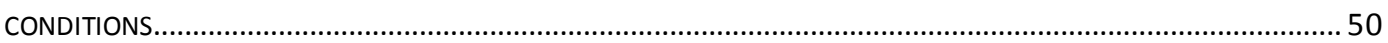

3.1.1 Get3 - A redox-regulated chaperone in eukaryotes ............................................................. 51

3.1.2 Mechanism of Get3's a ctivation process ............................................................................5 57

3.1.3 Activation of Get3's chaperone function is a fully reversible process in vitro ...................... 63

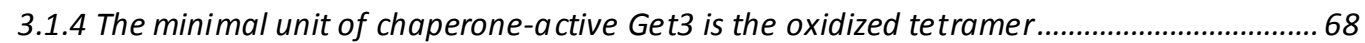

3.1.5 Oxidative activation of Get3 causes massive structu ral rearrangements ........................... 73

3.1.6 Dissecting the two Get3 functions in vivo ........................................................................... 79

3.1.7 Get3 colocalizes with unfolding proteins du ring oxidative stress in vivo ............................. 83

3.2 THE ATP-INDEPENDENT CHAPERONE GET3 PROTECTS SOLUBLE PROTEIN UNDER OXIDATIVE STRESS CONDITION IN

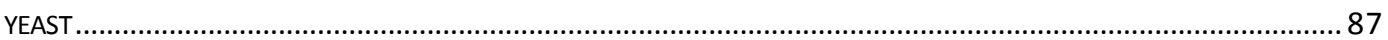

3.2.1 Oxidized Get3 maintains its substrate in a folding competent conformation under

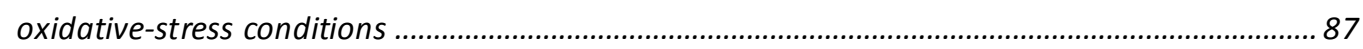

3.2.2 Visualizing the Get $3_{\text {tet }}-$ lu ciferase complex .................................................................... 94

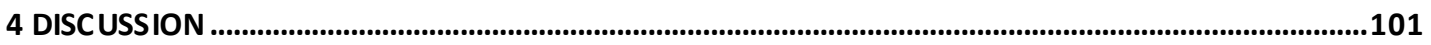

4.1 GET3, A PROTEIN WITH A DUAL FUNCTION AS TA-PROTEIN TARGETING FACTOR AND GENERAL CHAPERONE.....102

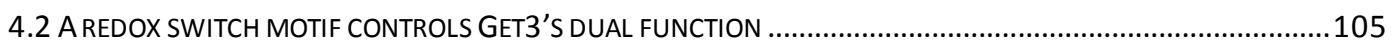

4.3 REVERSIBLE UNFOLDING AND OLIGOMERIZATION EXPOSES CLIENT BINDING SITES IN CHAPERONE ACTIVE GET3 108

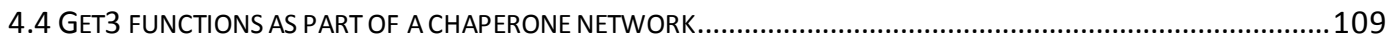

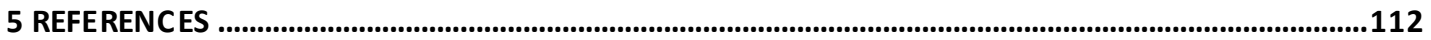

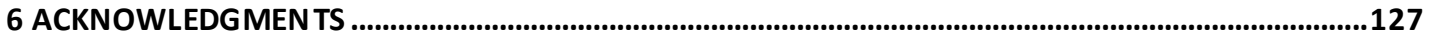

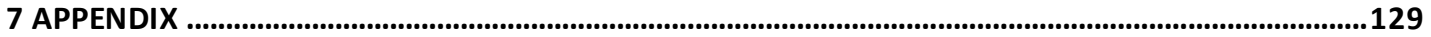

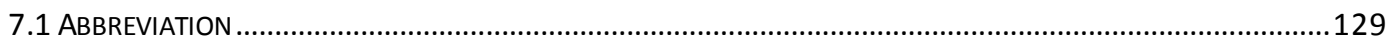

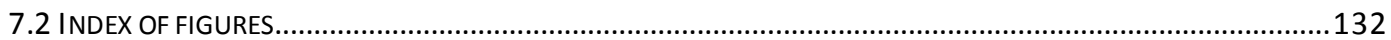


Index of Contents

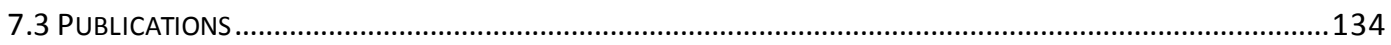

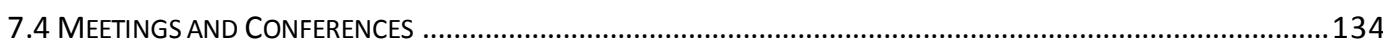




\section{Introduction}

\subsection{Protein homeostasis - A life-long challenge}

From the moment a nascent polypeptide chain emerges from the ribosome until it adopts its fully folded and functional state, a protein is constantly challenged by various intracellular and extracellular stressors. Particularly reactive oxygen species (ROS), such as superoxide radicals $\left(\mathrm{O}_{2 \cdot{ }^{-}}\right)$, hydrogen peroxide $\left(\mathrm{H}_{2} \mathrm{O}_{2}\right)$ or hydroxyl radicals $(\mathrm{OH} \cdot)$, which are constantly generated during normal aerobic metabolism (e.g. by the respiratory chain) can cause significant cellular damage including the unfolding and aggregation of essential proteins (Drose \& Brandt 2012, Imlay 2013). Many human diseases, including Alzheimer, Huntington, and diabetes as well as the aging process itself are directly connected to this oxidative stressmediated macromolecular damage (Broadley \& Hartl 2009, Maritim et al 2003, Romano et al 2010).

The cellular proteostasis network, which consists of many different chaperones, folding catalysts and proteolytic components maintains proteome stability and functionality during non-stress and stress conditions (Bukau et al 2006, Roth \& Balch 2011). A specific set of molecular chaperones are considered "foldases" since they facilitate the folding of nascent polypeptide chains, bind unfolding proteins to prevent aggregate formation and refold them once the stress has subsided. This group of chaperones is comprised of members of the heat shock protein (Hsp) family, including Hsp90 (E. coli HtpG), Hsp60 (E. coli GroEI), Hsp70 (E. coli DnaK) and Hsp100 (E. coli ClpB). They all utilize ATP binding and hydrolysis to support folding of newly synthesized polypeptides and/or refolding of damaged or even aggregated proteins (Figure 1.1) (Bukau et al 2006, Hartl 2011, Liberek et al 2008). The second arm of the proteostasis network consists of ATP-dependent proteases that degrade irreversibly damaged proteins (Jung \& Grune 2013, Jung et al 2013). The expression of molecular chaperones and proteases is under heat shock control and can be induced by the accumulation of protein-unfolding intermediates (Nonaka et al 2006, 
Verghese et al 2012).

Transcriptional regulation and the synthesis of new chaperones, however, require time and energy, thus delaying the response towards fast-acting protein unfolding conditions, such as severe oxidative stress. Moreover, accumulation of ROS causes a massive decrease in cellular ATP levels, which limits the function of existing ATP-dependent chaperones and impairs the synthesis of new chaperones (Colussi et al 2000, Osorio et al 2003, Winter et al 2005). Therefore, as a first line of defense to combat sudden oxidative stress, organisms employ redox-regulated chaperones, which use structurally or functionally important redox-sensitive cysteines to sense the oxidants (Antelmann \& Helmann 2011, Groitl \& Jakob 2014). Reversible oxidation of these cysteines causes a rapid change in structure and function of these proteins, turning them into active ATP-independent chaperones that prevent the accumulation of protein aggregates. In prokaryotes, one of these redox-regulated chaperones is Hsp33 (Jakob et al 1999). The Hsp33 protein becomes specifically activated by hypochlorous acid $(\mathrm{HOCl})$ stress, a physiological antimicrobial that causes widespread protein unfolding and aggregation (Winter et al 2008). $\mathrm{HOCl}$ stress is also involved in the activation of the redox-regulated chaperone function of RidA ( $E$. coli). However, its reversible activation involves a novel mechanism, $\mathrm{HOCl}$ treatment of RidA mediates the $N$-chlorination of its positively charged residues, which leads to an increased surface hydrophobicity and hence promotes its binding to unfolded cytosolic proteins (Muller et al 2014). Other members of ATP-independent chaperones are activated by various distinct stresses, e.g., small heat shock proteins (sHsps) like prokaryotic lbpA/lbpB (E. coli) or the eukaryotic Hsp26 (yeast) become active under heat stress conditions, whereas HdeA in E. coli is activated by acid stress (Haslbeck et al 2005a, Matuszewska et al 2009, Tapley et al 2009). All these stressactivated chaperones bind unfolding client proteins and keep them in solution until the stress levels off. 


\subsection{Molecular chaperones and protein homeostasis}

Molecular chaperones maintain functionality of the cell by assisting the folding, assembly and disassembly of polypeptide chains (Bukau et al 2006). They do not accelerate folding and typically dissociate from the client once folding is completed. The binding of chaperones to folding intermediates, especially to hydrophobic segments, reduces the concentration of aggregation-sensitive early folding intermediates and thus prevents their aggregation (Hartl et al 2011, Jahn \& Radford 2008). Under stress conditions that cause widespread protein unfolding (e.g., heat shock), cells react with the heat shock response by highly up-regulating the expression of molecular chaperones (Ellis 2006, Verghese et al 2012). Chaperones are divided into at least two functional categories; ATPdependent chaperone foldases and ATP-independent, often stressactivated chaperone holdases (Figure 1.1).

\subsubsection{Expression of molecular chaperones is controlled by the heat shock response}

The expression levels of most molecular chaperones are tightly controlled, allowing the cell to cope with the constant challenges that endanger the proteome and maintain fitness. Environmental changes or reduced access to nutrients, however, can rapidly impair proteostasis and overwhelm the chaperone network. Especially bacteria must withstand countless environmental insults, like the acidic environment of the stomach or ROS produced by the cells of the innate host defense, to survive within and infect their hosts (Klebanoff 2005, Lin et al 1995).

Stress-induced protein unfolding conditions in E. coli trigger the transcriptionally regulated heat shock response (HSR). The main player of the bacterial HSR is the heat shock sigma factor sigma $32\left(\sigma^{32}\right)$, which controls expression of most molecular chaperones and proteases in the cell (Nonaka et al 2006). Under non-stress conditions, $\sigma^{32}$ degradation is constantly mediated by the DnaK/J/E and GroEL/ES chaperones (Guisbert et al 2004). Upon shift to higher temperatures, the unfolding proteins titrate 


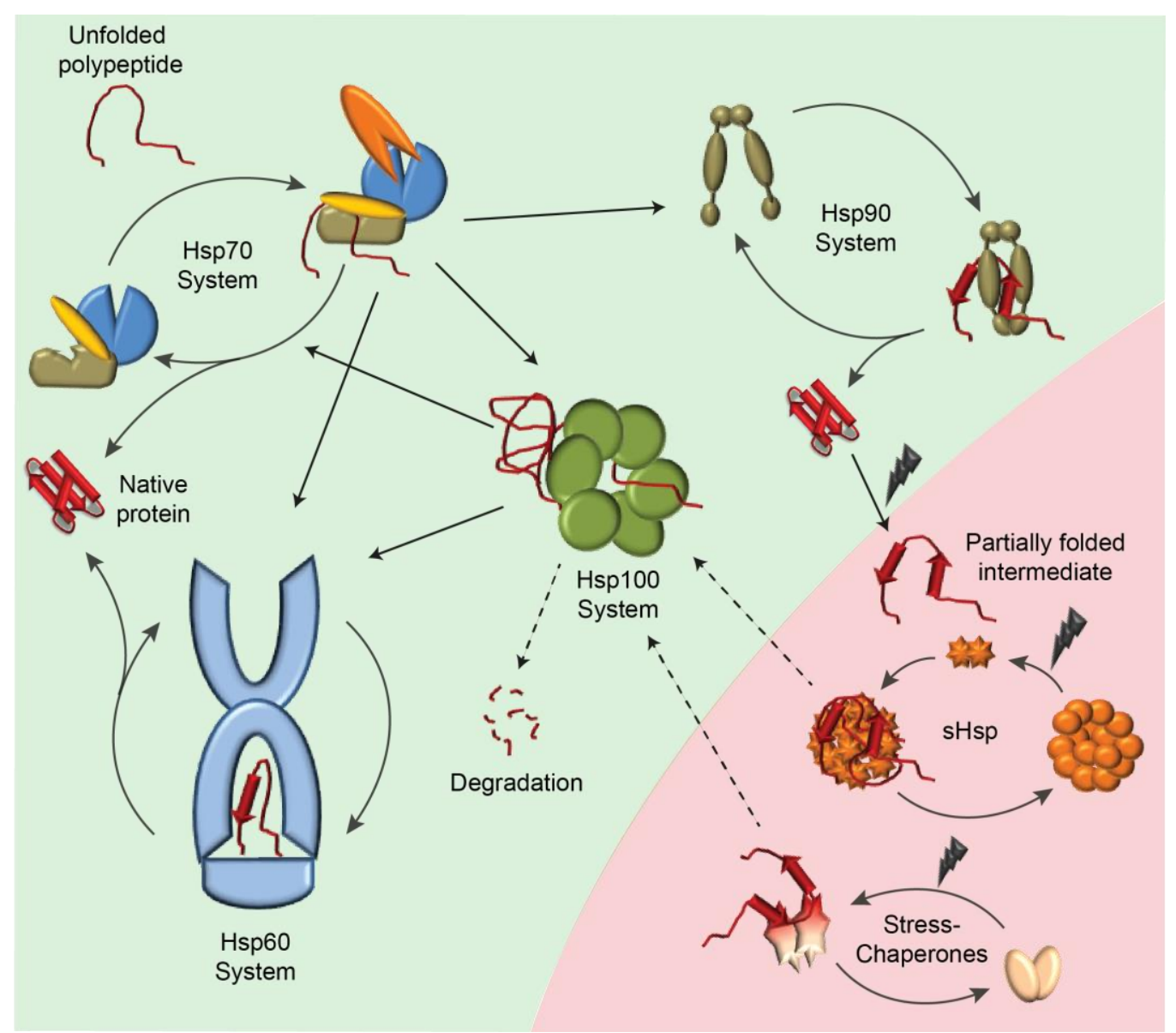

Figure 1. 1: A complex chaperone network maintains the proteostasis in the cell Schematic illustration of the multi chaperone network that promotes protein folding, maintains protein function and prevents protein aggregation in the cell. The large class of ATP-dependent chaperones (green shaded area) that can be found in different compartments of the cell utilize ATP to undergo cycles of structural rearrangements coupled to protein binding and folding. The heat shock protein 70 (Hsp70) system that is composed of Hsp40 (orange) and the multi-domain protein Hsp70 (blue, yellow, green) bind to new unfolded polypeptide chains emerging from the ribosome and mediate their partial or native folding. Partially folded proteins are then recognized by Hsp90 that mediates their folding into the native structure. Cytosolic Hsp60 (chaperonin), a huge multi-subunit complex shown in blue, mediates the folding of unfolded or partially folded protein intermediates to native structure. Substrate can undergo multiple rounds of folding at the respective chaperone system to reach the native protein's structure. Misfolded or partially aggregated proteins can be "recycled" via the Hsp100 system, it actively promotes unfol ding or disaggregation of proteins. Successfully unfolded protein can undergo a new round of folding whereas irreversibly aggregated proteins are triaged for degradation. Stress-induced protein unfolding conditions (black flash) deplete ATP levels in the cell, thus shutting down the ATP-dependent chaperone network. ATPindependent chaperones (in the red shaded area) are now activated by heat induced unfolding, oxidation of the redox-switches which mediate structural rearrangements (Stress-Chaperones) or dynamic oligomerization processes (small (s)Hsp) that uncover mostly hydrophobic substrate binding sites. They interact with partially folded substrate and prevent its aggregation. Upon return to non-stress conditions and to recovering ATPlevels, substrate is transferred to ATP-dependent chaperones for refolding or degradation. 
free chaperones and the $\sigma^{32}$ protein becomes stabilized (Guisbert et al 2004, Nakahigashi et al 1999). The induction of $\sigma^{32}$ synthesis is further controlled by the secondary structure of its mRNA which serves as a thermosensor. At high temperatures, the mRNA melts and facilitates ribosome binding, resulting in enhanced $\sigma^{32}$ biosynthesis (Morita et al 1999). The cellular ratio between free DnaK/J/E and GroEL/ES chaperones and $\sigma^{32}$ allows a tight feedback regulation (Guisbert et al 2004, Matsui et al 2008).

In eukaryotes, the HSR is also a highly conserved transcriptional cascade, triggered by the heat shock factor (HSF). Encounter of thermal and other environmental stresses result in the expression of cytoprotective genes as well as genes inducing cell cycle arrest (Morano et al 2012, Rowley et al 1993, Verghese et al 2012). Inactive under non-stress conditions, HSF1 undergoes rapid oligomerization and phosphorylation under heat stress conditions turning it into an active transcriptional regulator(Goodson \& Sarge 1995, Holmberg et al 2001). In the active form, HSF1 binds to heat shock elements (HSEs) in the promotor region of heat shock genes (Akerfelt et al 2010, Sakurai \& Takemori 2007). Similar to prokaryotes, HSF1 activity is controlled by chaperones. Under non-stress conditions, the Hsp70 and Hsp90 chaperone systems repress HSF1 activity. These chaperones are titrated away by unfolding proteins, and set HSF1 free to oligomerize and activate as transcription factor (Matsumoto et al 2005, Voellmy \& Boellmann 2007).

\subsubsection{ATP-dependent chaperones}

ATP-dependent chaperones are mainly involved in the folding of newly synthesized polypeptide chains and assembly of subunits to multi-protein complexes. They can be found in the cytosol of prokaryotes or in various compartments in the eukaryotic cell. Here, they utilize rounds of ATP binding and hydrolysis to undergo cycles of structural rearrangements which are coupled to protein binding and release, thereby supporting the folding of their substrates (Ellis 2006, Hartl et al 2011, Young et al 2004). 


\subsubsection{Hsp70 chaperone folding machinery}

The Hsp70 chaperone family comprises a universal class of proteins that play a central role in protein folding and proteostasis across all species. Hsp70 (DnaK in E. coli) together with its co-chaperone Hsp40 (DnaJ) is involved in quality control of newly made and preexisting polypeptide chains (Bukau et al 2006, Mayer 2013). Multiple homologues of this protein are present in most living organisms. The ATP-dependent chaperone foldase DnaK preferably binds to exposed short hydrophobic, unstructured regions of newly synthesized polypeptides or unfolded client proteins thus preventing their misfolding and promoting their correct folding (Daugaard et al 2007, Reichmann et al 2012, Rudiger et al 1997). Apart from protein folding during biogenesis, members of the Hsp70 family play roles in mitochondrial import, protein degradation and protein refolding after stress (Daugaard et al 2007, Petrucelli et al 2004, Young et al 2003, Zolkiewski et al 2012).

Hsp70 proteins consist of two major domains, an N-terminal nucleotide binding domain (NBD) and a C-terminal substrate binding domain (SBD) connected via a highly conserved linker segment (Bertelsen et al 2009, Kumar et al 2011, Mayer 2013). The transient interaction with substrates is regulated by ATP binding and hydrolysis in the NBD (Mayer 2013). The flexible linker domain allows allosteric coupling between the NBD and SBD and hence links nucleotide turnover to client protein-binding (Kumar et al 2011, Vogel et al 2006). In the ATP-bound open conformation, the overall affinity to substrate is low, while in the nucleotide-free or ADP bound closed conformation, Hsp70 exhibits high affinity for its substrates (Figure 1.2) (Bertelsen et al 2009, Mayer 2013, Mayer et al 2001). Crystallization studies on Hsp70 in complex with bound peptide revealed the substrate binding pocket in a closed conformation. It consists of a $\beta$-sandwich covered by an $\alpha$-helical lid, forming a hydrophobic cleft of $\sim 5$ residues as illustrated in Figure 1.2 (Bertelsen et al 2009, Popp et al 2005, Zhu et al 1996). The repeated cycles of substrate binding and release ensure proper client folding and prevent unfolded substrate from aggregating. 


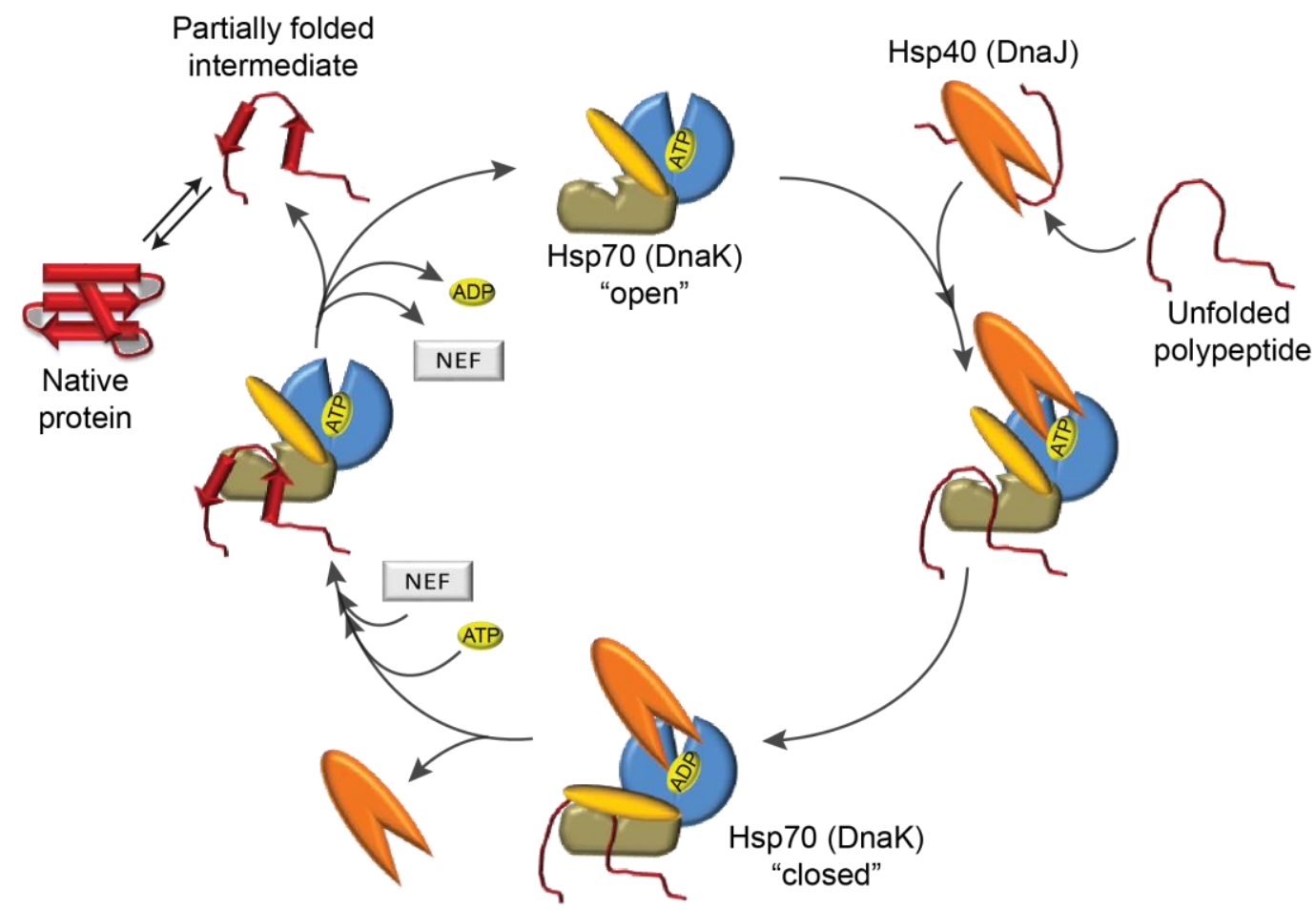

Figure 1. 2: The Hsp70 chaperone cycle

Schematic representation of the heat shock protein 70 (Hsp70) cycle (modified from Mayer 2013). Hsp70 chaperones (DnaK in E. coli) consist of three domains, the nucleotide-binding domain (NBD) shown in blue, the substrate-binding domain composed of a $\beta$-sandwich subdomain (SBDb) in greenand a $\alpha$-helical subdomain (SBDa) in yellow. ATP-bound Hsp70 is in the "open" conformation and only weakly interacts with unfolded substrates (red). The Hsp40 (DnaJ) co-chaperone recruits unfolded substrate to Hsp70 and stimulates its ATP hydrolysis, which induces a conformational change and stabilizes substrate binding in the SBD. In the ADP-bound "closed" conformation the substrate is trapped in a binding pocket between SBDb and SBDa, allowing parts of the substrate to fold. A nucleotide exchange factor (NEF) stimulates nucleotide exchange in Hsp70 and hence returns it to the low-affinity ATP-bound conformation triggering substrate release. The substrate can now refold to the native conformation or bind again to $\mathrm{Hsp} 70$ and undergo a repeated cycle of folding.

The intrinsic ATPase activity of Hsp70 $\left(<0.1 \mathrm{~s}^{-1}\right)$ that couples the substrate binding-release cycle is too slow to compete with the fast aggregation of protein substrates (Chang et al 2008, Schroder et al 1993). To facilitate this process, Hsp70 interacts with co-chaperones, particularly the J-protein Hsp40 (E. coli DnaJ) and nucleotide exchange factors (NEFs) like the GroPlike gene $E(\mathrm{GrpE})$ in prokaryotes or BAG1, which is one of many NEFs in eukaryotes (Bracher \& Verghese 2015, Greene et al 1998). The interaction of Hsp40 with Hsp70's NBD stimulates the ATP to ADP conversion, 
stabilizing the substrate-bound form and locking bound substrate in the SBD (Ahmad et al 2011, Greene et al 1998, Suzuki et al 2010). Thus, J-proteins play a critical role in regulating the Hsp70 chaperone cycle in the cell.

The Hsp40 family $(\sim 40 \mathrm{kDa})$ is characterized by the presence of a highly conserved J-domain ( 70 amino acids), which mediates the interaction with Hsp70 (Kampinga \& Craig 2010, Mayer et al 1999, Rajan \& D'Silva 2009). J-proteins serve as holdases. They prevent aggregation of unfolded substrate and deliver client proteins to the open Hsp70 substrate cleft (Figure 1.2) (Kampinga \& Craig 2010, Mayer 2013, Schroder et al 1993). The number of J-proteins in a cell often exceeds the number of Hsp70 proteins, suggesting additional potentially Hsp70-independent functions. Humans, for instance have $11 \mathrm{Hsp} 70 \mathrm{~s}$, but $41 \mathrm{~J}$-proteins (Hageman \& Kampinga 2009). Although the C-terminal domains among various members of the Hsp40 family are highly variable, they are all able to interact with Hsp70. The current model suggests that the greater number of J-proteins and their variability confers client specificity, connecting Hsp70 and its multiple J-protein partners to various physiological functions in the cell (Fan et al 2004, Kampinga \& Craig 2010, Rajan \& D'Silva 2009).

The nucleotide exchange factor GrpE $(\sim 21 \mathrm{kDa})$ in $E$. coli accelerates the nucleotide exchange of DnaK (Hsp70) (Bracher \& Verghese 2015, Harrison 2003). Binding of GrpE lowers DnaK's affinity for ADP ( 200-fold) (Packschies et al 1997). This allows the rebinding of ATP and returns DnaK to the low-affinity binding state, which triggers substrate release (Figure 1.2) (Brehmer et al 2004, Mally \& Witt 2001). ATP rebinding leads to dissociation of GrpE from DnaK, thus initiating a new chaperone cycle (Brehmer et al 2004). There are several different NEFs in higher eukaryotes; however, their exact functions still need to be investigated (Bracher \& Verghese 2015). Notably, eukaryotic Hsp40/70 refolds substrate in the absence of a NEF in vitro (Haslbeck et al 2005b, Lee \& Vierling 2000).

\subsubsection{The Hsp60, Hsp90 and Hsp100 chaperone network}

Folding of complex multimeric proteins requires a specialized class of 
chaperones, so called chaperonins (Hsp60), which form large oligomeric structures, which provide an insulated folding cage that minimizes entropy and maximizes folding potential to facilitate the folding of complex threedimensional protein structures towards their native state (Figure 1.1) (Spiess et al 2004). These oligomeric complexes of $\sim 800 \mathrm{kDa}$ are composed of two heptameric or octameric (cis and trans) rings of identical or closely related rotationally symmetric subunits in prokaryotes (GroEL, Hsp60) or eukaryotes (TriC), respectively (Horwich et al 2007, Pereira et al 2010, Xu et al 1997). Chaperonins are divided into two structurally similar subgroups (Horwich et al 2007); group I chaperonins are found in the cytosol of prokaryotes (GroEL) or in eukaryotic organelles such as chloroplasts or mitochondria (Hsp60). Group II chaperonins are found in the eukaryotic (TRiC) or archeal cytosol. Both subgroups undergo fairly similar ATP-driven reaction cycles, and undergo large conformational changes that bury the hydrophobic substrate-interaction sites, thus allowing unfolded substrate to enter the central cage-like structure of the chaperonin (Figure 1.1) (Horwich et al 2006, Pereira et al 2010, Spiess et al 2004). ATP-binding to the cis ring in GroEL simultaneously allows substrate to enter the folding cage and GroES binding to enclose the compartment (Horwich et al 2006). ATPhydrolysis sets the timer for folding of the polypeptide. Upon binding ATP to the trans ring GroES dissociates and folded substrate is released form the folding cage (Rye et al 1999). In TRiC mediated folding additional partner proteins ensure that only folded proteins exit the folding compartment (Spiess et al 2004). Substrates can undergo multiple rounds of folding to reach the native folded state. The exact mechanism by which the chaperonins promote protein folding is focus of intense research.

The two groups of chaperonins differ in the mechanism by which substrates are encapsulated. While group I chaperonins employ the co-chaperone GroES (11 kDa), which forms a dome-shaped heptameric "lid", group II chaperonins utilize flexible $\alpha$-helical extensions that act as a substratebinding site and cover the folding cage (Hunt et al 1996, Llorca et al 1999). Moreover, TRiC, with the assistance of upstream chaperones, can bind cotranslationally to nascent chains as they emerge from the ribosomes 
(Siegers et al 2008).

Another class of highly conserved ATP-dependent chaperones is the Hsp90 family, which consists of $\sim 90 \mathrm{kDa}$ chaperones that can be found in the cytosol, the endoplasmic reticulum (ER) and the mitochondrial matrix, accounting for $1-2 \%$ of all cellular proteins (Finka \& Goloubinoff 2013, Wandinger et al 2008). Unlike Hsp60 or Hsp70, Hsp90 does not bind nascent polypeptide chains but assists proteins at a later stage of folding (Figure 1.1) (Jakob et al 1995, Young et al 2004). In eukaryotic cells, Hsp90 is essential; it assists in the folding of protein kinases and transcription factors. Moreover, it facilitates steroid hormone receptor maturation, and hence it is involved in signaling (Dittmar \& Pratt 1997, Miyata \& Yahara 1995, Tao \& Zheng 2011). All Hsp90 family members form a constitutive homodimer connected through its $\mathrm{C}$-terminal dimerization domain, followed by a conserved and structurally flexible middle domain that connects to the $\mathrm{N}$-terminal ATPase domain (Ali et al 2006, Didenko et al 2012). ATP binding induces closing of the "molecular clamp" like structure of the N-terminal domain, which is essential for ATP hydrolysis and fosters the formation of a potential substrate binding site in the middle domain (Blacklock \& Verkhivker 2014, Krukenberg et al 2009, Shiau et al 2006). In the ATP-bound state, Hsp90 binds to substrate and releases it upon ATP hydrolysis (Pearl et al 2008). Cytosolic Hsp90 in eukaryotes interacts with a variety of cochaperones, which form a multi-chaperone complex and regulate Hsp90's activity (Wandinger et al 2008, Zuehlke \& Johnson 2010).

Stress conditions can impair and overload the classic folding chaperones. This results in the accumulation of misfolded proteins and irreversible protein aggregates. A specialized class of chaperones, i.e. Hsp100 is able to dissolve misfolded or aggregated protein upon return to non-stress conditions in an ATP-dependent manner, and restore protein homeostasis (Doyle \& Wickner 2009). Hsp100's like E. coli ClpB or yeast Hsp104 are members of the AAA+ superfamily of ATPases, which are associated with various cellular activities such as metal chelation and DNA repair (Fodje et al 2001, Gribun et al 2008). They typically function in a hexameric ring 
arrangement with a central channel (Figure 1.1), stabilized by ATP binding to the N-terminal domain of each Hsp100 monomer (Doyle et al 2013, Lee et al 2010, Lee et al 2003). A current model is that Hsp104/ClpB together with the Hsp70/DnaK chaperone system act synergistically to extract of unfolded polypeptides from aggregates and promote their subsequent refolding (Haslbeck et al 2005b, Schlieker et al 2004, Zolkiewski et al 2012). However, it is yet not clear how the labor is divided between the two systems (Doyle \& Wickner 2009). Another subset of Hsp100's (E. coli ClpX together with $(\mathrm{lpP})$ is involved in proteasomal degradation of insoluble protein aggregates to prevent the nucleation of inactive complexes (Sauer et al 2004).

\subsubsection{ATP-independent chaperones}

Most ATP-independent chaperones work as chaperone holdases and lack refolding activity. They are strongly induced by a variety of different protein unfolding stress conditions but also function constitutively in multiple cell types and organisms. For some ATP-independent chaperones in eukaryotes like human aB-crystallin or yeast Hsp26, it has been shown that increased temperature directly induces conformational changes promoting chaperone activity (Haslbeck et al 2005a). Another subset of stress-induced chaperones utilizes changes in ROS levels to undergo structural rearrangement that activate their chaperone holdase activity, allowing instant response to ROS stress (Jakob et al 1999, Kumsta \& Jakob 2009). These chaperone holdases bind tightly to their client proteins and often depend on ATP-dependent foldases for client refolding once stress conditions subside. In most cases, activation of these chaperones is triggered by stress-induced unfolding, which exposes an otherwise buried client binding site (Basha et al 2012, Graf et al 2004, Reichmann et al 2012).

\subsubsection{Small heat shock proteins fight protein aggregates}

The family of small heat shock proteins (sHsp) forms a very diverse group of chaperones that play an important role in maintaining protein quality in all kingdoms of life. They vary strongly in sequence, structure and size, but 
rarely exceed $\sim 40 \mathrm{kDa}$. sHsps form highly dynamic oligomers $<600 \mathrm{kDa}$, that continuously dissociate into smaller units that can re-associate again. One common feature of sHps's is the presence of a conserved $\alpha$-crystallin domain of $\sim 90$ amino acids (Basha et al 2012, Haslbeck \& Vierling 2015, White et al 2006) flanked by the $\mathrm{N}$-terminal region and a C-terminal extension (Haslbeck et al 2005a, Sun \& MacRae 2005). Structural analysis revealed that the $\alpha$-crystallin domain consists of two anti-parallel sheets of three and four $\beta$-strands, respectively connected by a short inter-domain loop, forming a $\beta$-sandwich (Delbecq \& Klevit 2013, Van Montfort et al 2001). Although low in sequence identity, the formation of a $\beta$-sandwich is an evolutionarily conserved hallmark of the sHsp (Basha et al 2012, Kriehuber et al 2010, Poulain et al 2010). The $\alpha$-crystallin domain can form a dimer mediated by the formation of an inter-subunit composite $\beta$-sheet (Bagneris et al 2009). Dimeric sHsp is the smallest unit. to form higher oligomeric complexes, extensions of the $\mathrm{N}$-terminal regions intertwine to form pairs of a knot-like structure stabilized by hydrophobic interactions (Basha et al 2012). Concurrently, a conserved $W / L-X-I / N / L$ motif in the C-terminal extension anchors itself in a hydrophobic groove of a neighboring chaperone dimer (Jehle et al 2011, Poulain et al 2010).

Unlike ATP-dependent chaperones, sHsp do not promote folding. They form a first line of defense under stress conditions by tightly binding unfolding substrates and keeping them soluble until the stress subsides. However, sHsps are not able to rescue already aggregated proteins or interact with native substrate proteins (Poulain et al 2010). Intriguingly, while some sHsp oligomers dissociate into stable dimers at elevated temperature to bind unfolded substrate, others retain the oligomeric structure that comprises the chaperone active species (Haslbeck et al 2005a, Haslbeck et al 1999, Stengel et al 2010). However, heat-induced oligomer dissociation, accompanied by uncovering of hydrophobic residues that increase surface hydrophobicity and expose a substrate binding sites is thought to be the major activation mechanism of sHsps (Haslbeck et al 2004, Haslbeck et al 2005a, Plater et al 1996). Smaller activated sHsp units can again reassociate either to a chaperone-active oligomer or when already occupied 
with substrate to an oligomeric substrate complex, which was observed for yeast sHsp26 (Franzmann et al 2005, Stengel et al 2010, Stromer et al 2003). Refolding of substrates bound to sHsp requires the presence of ATPdependent chaperones. In yeast, for instance, the Hsp70 system either alone or in combination with the disaggregase Hsp104 promotes the release and refolding of substrate bound to sHsp26 (Haslbeck et al 2005b). This chaperone cycle allows organisms to rapidly react to protein unfolding conditions and stabilize their proteome without the need for energyconsuming new protein synthesis.

Crosslinking studies showed that substrates interact with the N-terminal domain of sHsps, suggesting that the hydrophobic patches in the flexible $\mathrm{N}$ terminal domain might represent the substrate binding site in sHsp (Jaya et al 2009, Patel et al 2014). Hydrogen-deuterium exchange experiments indicate that this region completely lacks stable secondary structure, suggesting that the $\mathrm{N}$-terminal domain might be intrinsically disordered (Cheng et al 2008, Jaya et al 2009, Wintrode et al 2003). Recently, a new model in which ATP-independent chaperones use internal disorder that allows large flexibility in substrate recognition to bind general unfolded substrate was proposed (Reichmann et al 2012), suggesting that sHsps might use their intrinsically disordered regions to bind substrate in a manner akin to conditionally disordered chaperones.

\subsubsection{Conditionally disordered chaperone}

Unfolding coupled to an increase in chaperone activity is a common feature among conditionally disordered chaperones. These chaperones are fully folded and chaperone-inactive under non-stress conditions, but rapidly adopt a partially disordered conformation when exposed to distinct stress conditions (Bardwell \& Jakob 2012). The disordered regions seem to be essential in the binding of aggregation-prone client proteins and allow an increased flexibility in client binding (Reichmann et al 2012). This new chaperone category includes yeast Hsp26, bacterial Hsp33 and the more recently discovered HdeA (Haslbeck et al 2005a, Reichmann et al 2012, Tapley et al 2009). While sHsps use heat-induced structural changes to 
expose their disordered regions for substrate binding, E. coli HdeA (9.7 kDa), a periplasmic chaperone, senses a drastic decrease in the $\mathrm{pH}$ of the environment to partially unfold into its chaperone active form (Franzmann et al 2005, Stromer et al 2003, Tapley et al 2009). HdeA, chaperone-inactive and dimeric at neutral $\mathrm{pH}$, when exposed to acidic environment (e.g. in the human stomach $(\mathrm{pH}<3)$ ) becomes partially unfolded and dissociates into chaperone-active monomers (Tapley et al 2009). In this partially disordered conformation, it can adopt various conformations to bind a broad range of substrates (Tapley et al 2010, Tapley et al 2009). Another chaperone whose redox-mediated activation is accompanied by substantial unfolding is Hsp33. Its extensive characterization was the cornerstone for the establishment of the class of conditionally disordered chaperones (Reichmann et al 2012).

\subsubsection{Oxidative stress challenges the proteostasis network}

Oxidative stress conditions that cause protein unfolding add several layers of complexity to the proteostasis machinery. Oxidants are very fast acting, whereas transcriptional regulation and synthesis of new chaperones require a certain response time. Moreover, many of the proteins involved in transcription and translation process are oxidation-sensitive themselves, and become transiently inactivated by oxidants or protein unfolding conditions (Brandes et al 2009, Maret 2006, Winter et al 2005). An additional challenge is the substantial drop in cellular ATP levels caused by oxidative stress (Colussi et al 2000). This further limits the ATP-dependent chaperone and protease network (Winter et al 2005). In eukaryotes it is thought that oxidative inactivation of the central player of glycolysis, glyceraldehyde dehydrogenase (GAPDH), together with the oxidative modification of other energy-generating systems causes the drop in ATP levels (Cotgreave et al 2002, Le Moan et al 2006, Leichert et al 2008). Recent studies in E. coli, however, demonstrated that the decline in ATP levels upon HOCl-stress is caused by the active re-routing of ATP into long chains of inorganic phosphate (Gray et al 2014). This raised the question as to the mechanism by which cells defend themselves against the very sudden exposure to 
highly proteotoxic oxidants such as $\mathrm{HOCl}$ or $\mathrm{OH}$. Eukaryotic and prokaryotic cells employ a subset of stress-specific ATP-independent chaperones that are regulated on the protein level and allow an instant stress-response. These chaperones, which are inactive under non-stress conditions, directly sense protein unfolding conditions, and rapidly react with an increase in their chaperone function (Graumann et al 2001, Tapley et al 2009). A major part of the current knowledge about ATP-independent and stress-induced chaperones derived from studies of the bacterial stress-response. Due to their "infective" lifestyle, these microorganisms constantly fight environmental stresses and hence developed a variety of efficient stressinducible chaperones. In addition, some bacterial compartments such as the periplasm are devoid of ATP, thus requiring a set of ATP-independent chaperones, which are subjects of recent studies(Lennon et al 2015, Quan et al 2011, Tapley et al 2009).

\subsubsection{The redox-regulated chaperone holdase Hsp33}

Hsp33, a $33 \mathrm{kDa}$ heat shock protein, is highly conserved in bacteria and some eukaryotic parasites such as Leishmania. It is constitutively expressed under non-stress conditions (Chuang \& Blattner 1993, Jakob et al 1999). The presence of four absolutely conserved cysteine residues involved in metal binding at the $\mathrm{C}$-terminus of the protein was a unique feature among all known Hsps. Due to the extensive work of the Jakob Lab during the past two decades, this zinc-coordinating cysteine motif was characterized as a universal posttranslational redox switch in proteins, connecting increased ROS levels to functional changes in proteins (Graf et al 2004, Groitl \& Jakob 2014, llbert et al 2007, Jakob et al 2000). Chaperone-inactive Hsp33 coordinates zinc via four thiol groups, which upon oxidation-induced disulfide bond formation and zinc release, turn Hsp33 into a potent chaperone holdase (Graumann et al 2001, llbert et al 2007, Jakob et al 2000). Activation of Hsp33 requires a combination of oxidative stress and increased protein unfolding that can be induced by elevated temperatures or bile salts (Cremers et al 2014, Cremers et al 2010, Graumann et al 2001). Bacteria encounter these stress conditions 


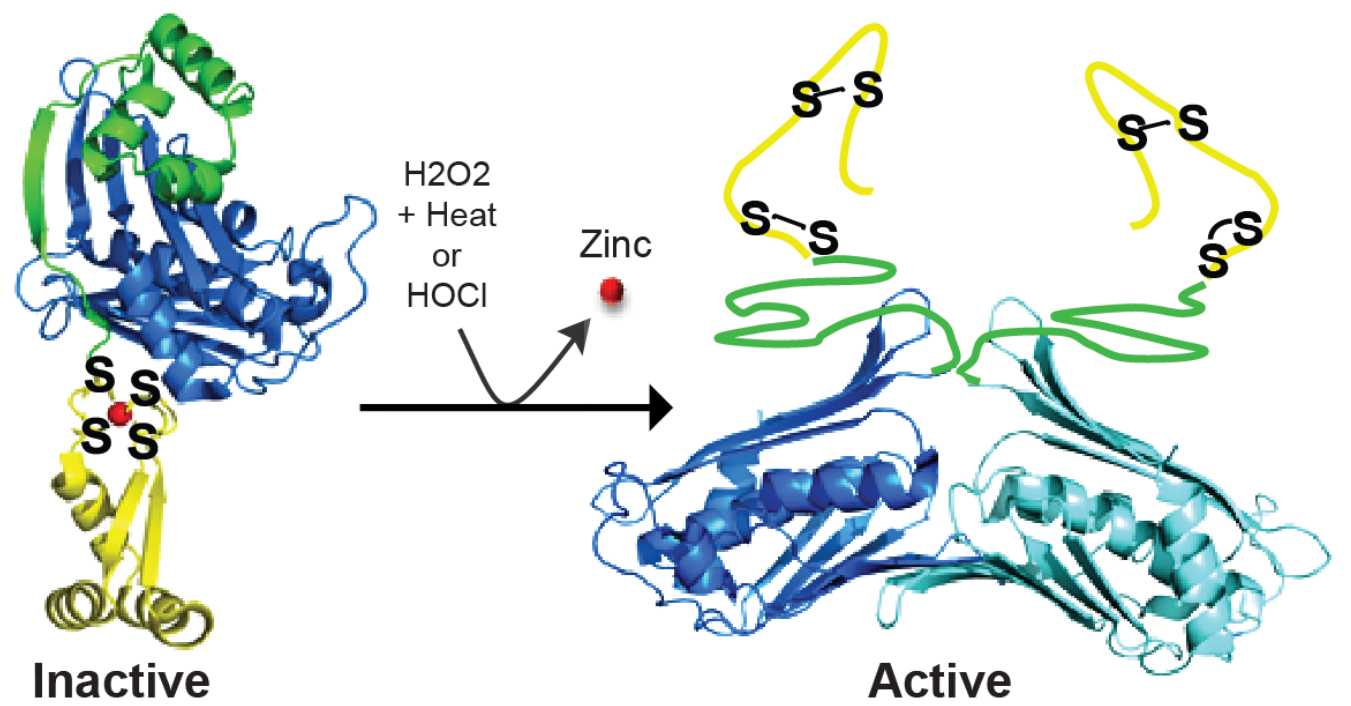

Figure 1. 3: Mechanism of Hsp33's chaperone activation

In its inactive form E. coli Hsp33 is a reduced and tightly folded monomer (combined from Protein Data Bank ID: 1I7F and 1XJH). The redox-sensing domain within the Cterminus (yellow) consists of four absolutely conserved cysteine thiols coordinating a zinc ion with high affinity. A flexible linker region (green) covers parts of the $\mathrm{N}$-terminal domain (blue) and connects the C-terminal domain to it. Consecutive oxidation of the for thiols groups accompanied by protein unfolding conditions (e.g. heat) leads to the formation of a two disulfide bonds, zinc release, dimerization and unfolding of the $\mathrm{C}$ terminal domainand linker region, presenting the hydrophobic surface of the $\mathrm{N}$-terminal platform. In the chaperone-active dimeric form (modified from Protein Data Bank ID: 1I7F), Hsp33 uses a combination of its partly disordered C-terminal domain and linker region together with the hydrophobic surface of the $\mathrm{N}$-terminal domain to bind unfolded client protein. Hypochlorous acid $(\mathrm{HOCl})$ induces protein unfolding and oxidative conditions simultaneously and is therefore a fast activator of Hsp33's chaperone-active form.

during fever or when passing through the gastrointestinal tract (Cremers et al 2014). Moreover, another fast activator of $\mathrm{Hsp33}$ is $\mathrm{HOCl}$, which simultaneously causes protein unfolding and strongly oxidative conditions (Winter et al 2008). $\mathrm{HOCl}$ is produced by cells of the innate immune response to kill bacteria (Miller \& Britigan 1997) or used as the active component of general disinfectants such as bleach.

Hsp33 consists of two domains, a compactly folded N-terminal domain (blue) and a C-terminal redox-sensing domain (yellow), connected by a flexible linker region (Figure 1.3, green). As shown in Figure 1.3, under reducing non-stress conditions the four absolutely conserved cysteines of 
the C-terminal domain arranged in a $\mathrm{C}_{232}-\mathrm{X}-\mathrm{C}_{234}-\mathrm{X}_{n}-\mathrm{C}_{265}-\mathrm{XY}-\mathrm{C}_{268}$ motif are engaged in the tetrahedral coordination of one $\mathrm{Zn}^{2+}$ ion (red sphere), stabilizing the C-terminal domain (Graf et al 2004, Janda et al 2004). The highly charged $\sim 52$ amino acid linker region, folded in three $\alpha$-helices, covers the largely hydrophobic four-stranded $\beta$-sheet platform of the $\mathrm{N}$ terminal domain (Figure 1.3) (Janda et al 2004, Vijayalakshmi et al 2001).

Under oxidative stress conditions, the distal cysteines Cys265/Cys268 engage in disulfide bond formation, release the zinc ion and induce unfolding of the C-terminal domain, which further destabilizes the upstream linker region and converts it into a thermolabile folding sensor (llbert et al 2007, Vijayalakshmi et al 2001). To activate this dynamic chaperone-inactive oxidation intermediate, a second stressor, namely protein unfolding conditions (e.g. elevated temperatures or bile salt), is required to shift the equilibrium of the linker region towards the fully unfolded conformation. Now, the proximal cysteines Cys232 and Cys234 are available for oxidation, forming a second disulfide bond, accompanied by unfolding of the linker region (Graf et al 2004, Graumann et al 2001, llbert et al 2007). Kinetically fast oxidants (e.g. $\mathrm{HOCl}$ ), however, rapidly form the second disulfide bond even in the absence of conditions leading to protein unfolding (Winter et al 2008). In the unfolded conformation, two oxidized Hsp33's dimerize and thus form a highly active chaperone (Figure 1.3).

Unfolding of the linker region exposes a hydrophobic surface in the $\mathrm{N}$ terminal platform. It was assumed that this area serves as a binding site for unfolded client proteins under stress conditions (llbert et al 2007). A more recent study now showed that oxidized, chaperone-active Hsp33 uses its own disordered linker region to interact with early protein-unfolding intermediates (Reichmann et al 2012). These results strongly suggest that apart from the $\mathrm{N}$-terminal platform, the flexible linker region is involved in substrate binding. Indeed, a new study using unnatural amino acids incorporated into Hsp33 to enable in vivo crosslinking and fluorine-19 nuclear magnetic resonance ( ${ }^{19} \mathrm{~F}$ NMR) spectroscopy, revealed a composite client-binding site that consists of polar residues from the flexible linker 


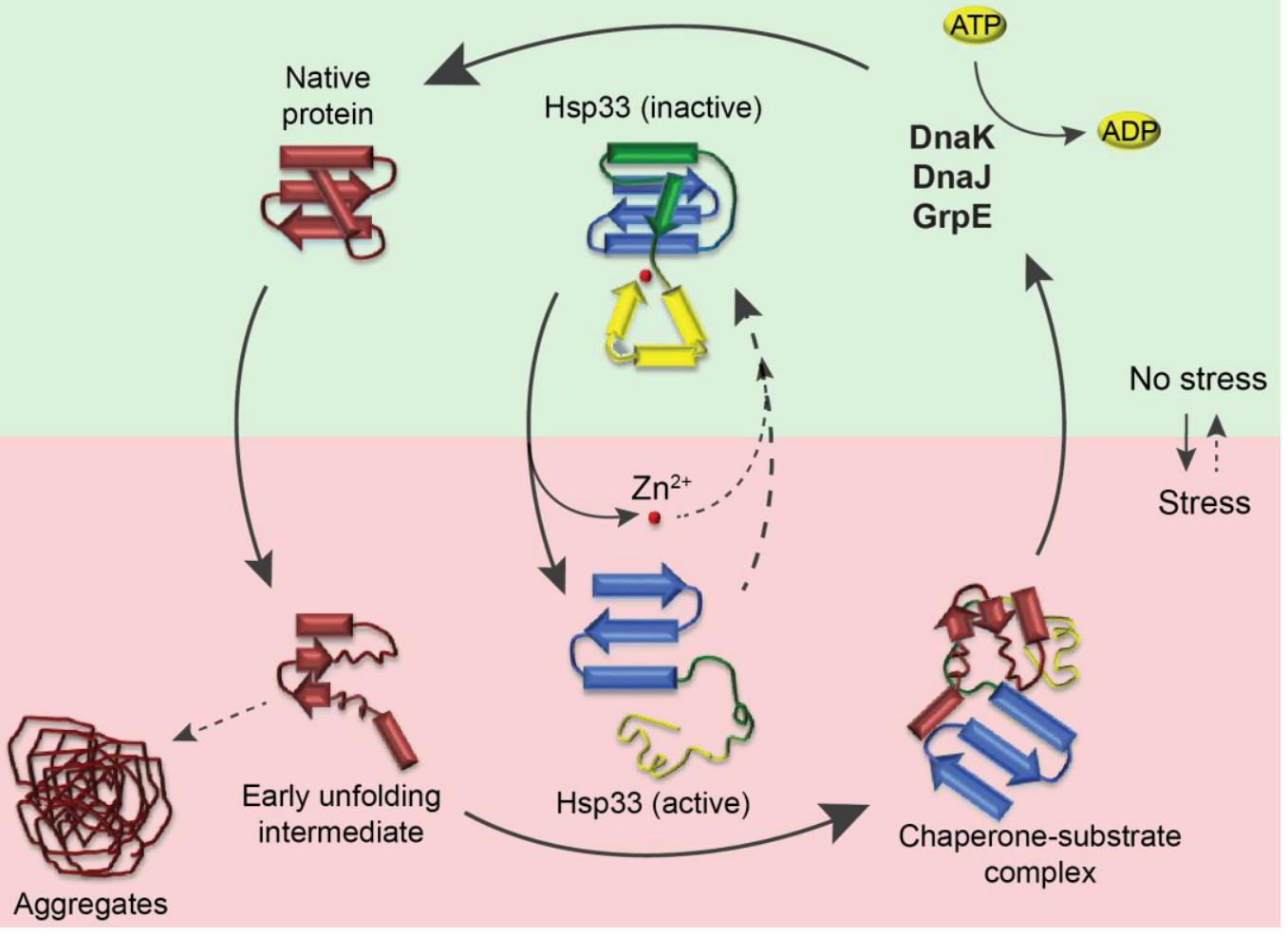

\section{Figure 1. 4: Hsp33's redox-mediated chaperone function}

Oxidative stress can induce substantial structural changes in proteins, causing protein unfolding and aggregation. Bacteria employ the inducible molecular chaperone Hsp33 to sequester unfolding intermediates and prevent accumulation of toxic protein aggregates. Under non-stress conditions, the C-terminal redox-sensing domain (yellow) and flexible linker region (green) of chaperone inactive Hsp33 are well folded, harboring a zinc ion coordinated by four reduced cysteine residues. Upon exposure to oxidative stress, protein unfolding conditions, and depleted ATP levels, Hsp33 undergoes oxidative disulfide bond formation, zinc release and massive structural rearrangements, including significant unfolding of the $\mathrm{C}$-terminal redox-sensing domain and flexible linker region, turning into chaperone active Hsp33. In this partially intrinsically disordered conformation, $\mathrm{Hsp33}$ is able to tightly bind early unfolding protein intermediates and prevent their aggregation. Upon return to non-stress refolding conditions, Hsp33's thiols become reduced. However, full inactivation of the chaperone functions requires the interaction with the DnaK/DnaJ/GrpE system, which receives the client protein and promotes full inactivation of Hsp33. The KJE system uses the now recovered ATP levels to refold the client proteins to their native structure.

region as well as nonpolar residues from the $\mathrm{N}$-terminal $\beta$-sheet platform surface of Hsp33 (Groitl et al 2016).

Hsp33 uses an intriguing activation mechanism that requires substantial unfolding of its secondary structure to gain chaperone function. Protein 
unfolding was previously thought to be detrimental for the function of most proteins. However, Hsp33 utilizes unfolding to activate a specific function, making it the founding member of a new category of so-called conditionally disordered chaperones (Bardwell \& Jakob 2012, Jakob et al 2014, Reichmann et al 2012).

The oxidative activation of Hsp33's chaperone function is fully reversible. Upon return to non-stress reducing and protein refolding conditions, oxidized Hsp33 dimers are reduced by the cellular glutaredoxin and thioredoxin system yet remain bound to their client proteins (Hoffmann et al 2004). Only when ATP levels are restored, ATP-dependent chaperone foldases composed of the bacterial DnaK/DnaJ/GrpE (Hsp70) system mediate the release of Hsp33's client proteins and its full inactivation (Hoffmann et al 2004, Reichmann et al 2012). Subsequently, the KJE system supports the refolding of client proteins to the native state.

No Hsp33 homologues are known in higher eukaryotes. Recent evidence now suggests that the highly conserved eukaryotic Get3 ATPase moonlights as a redox-regulated chaperone under ATP-depleting stress conditions, such as oxidative stress (Powis et al 2013b, Voth et al 2014).

\subsubsection{Peroxiredoxins exert a redox-dependent dual function}

Peroxiredoxins (Prxs) are conserved through all kingdoms of life. They detoxify ROS and reactive nitrogen species and are involved in $\mathrm{H}_{2} \mathrm{O}_{2}$ signaling and protein oxidation (Poole et al 2011, Rhee \& Woo 2011). In eukaryotes, cytosolic peroxiredoxins (Prxs) have been studied extensively in regard to their role in maintaining the redox balance in cells. More recently, however, a second function for 2-Cys-Prxs as molecular chaperones has been reported (Jang et al 2004, Rhee et al 2005).

2-Cys Prxs, like the bacterial AhpC or yeast cPrxl are obligate homodimers of two inverted subunits. They utilize their peroxidatic cysteine $(\mathrm{Cp})$ to directly react with peroxides forming a sulfenic acid and $\mathrm{H}_{2} \mathrm{O}$ in this process. The resolving cysteine $(\mathrm{Cr})$ of the other subunit reacts with the oxidized $\mathrm{Cp}$ and forms an intermolecular disulfide bond (Hirotsu et al 1999, Schroder et 
al 2000). Subsequently, this disulfide bond is reduced by oxidoreductases, such as thioredoxin (Chae et al 1994). Oxidation of Cp cysteine, which is located in the first turn of helix $\alpha 2$, induces the unfolding of the helix, a process that is required to form the intermolecular disulfide bond between Cr and Cp cysteines (Wood et al 2003a, Wood et al 2003b).

In vitro studies with 2-Cys-Prxs form yeast (cPrxl) revealed that overoxidation of the active cysteine $\mathrm{Cp}$ inactivates cPrxl's peroxidase function and induces the formation of high molecular weight (HMW) oligomers that prevent protein aggregation in an ATP-independent fashion (Jang et al 2004). Furthermore, the formation of HMW oligomers was shown to be fully reversible upon removal of $\mathrm{H}_{2} \mathrm{O}_{2}$ in vivo (Jang et al 2004). Other activation mechanisms for the functional switch from peroxidases to chaperones have been reported as well, including phosphorylation or exposure to low pH (Angelucci et al 2013, Jang et al 2006, Saccoccia et al 2012). A more recent study revealed that the mitochondrial tryparedoxinperoxidase mTXNPx from the protozoan parasite Leishmania infantum is activated as a chaperone holdase by temperature-mediated rearrangements within its reduced decameric structure (Taxiera et al. 2014). This study further reported that heat-unfolded substrates bound to mTXNPx are transferred to the prokaryotic Hsp70 chaperone foldase system for refolding (Teixeira et al 2015). This finding suggests that peroxidases in general could be a component of the cellular chaperone network maintaining protein homeostasis under stress conditions.

\subsection{The GET pathway is involved in the biogenesis of tail- anchored (TA)-Proteins}

Membranes of the endomembrane system of yeast and other eukaryotic cells contain integral membrane proteins, of which $\sim 3-5 \%$ display the topology of so-called tail-anchored (TA)-proteins (Beilharz et al 2003, Kalbfleisch et al 2007, Kriechbaumer et al 2009). These proteins are posttranslationally inserted into the membrane via a hydrophobic C-terminal transmembrane domain, hence the name. Members of the conserved Guided Entry of Tail-anchored proteins (GET)-system (Get1-5) target and 
guide TA-proteins after their synthesis at the ribosome to the endoplasmic reticulum (ER) membrane (Borgese et al 2003, Rabu et al 2009, Shao \& Hegde 2011). There, the TA proteins serve many essential cellular functions, including the regulation of apoptosis (e.g., Bcl-2/ Bcl-XL), mediation of intracellular trafficking (SNAREs) and facilitation of organelle biogenesis and secretion (Schuldiner et al 2008).

In addition to the GET-system, a small subset of TA-proteins such as synaptobrevin 2 (Syb2) appear to be also targeted to the ER-membrane by the GTP-dependent signal recognition particle (SRP) (Abell et al 2004). Moreover, members of the Hsp70 family (Hsc70) seem to recognize the TA sequence and mediate the insertion of specific TA-proteins, such as Sec61 $\beta$, into yeast membranes (Abell et al 2007, Rabu et al 2008). Most likely these mechanisms are closely intertwined. The dominant factor for TAprotein insertion, however, is thought to be the GET system with its central player Get3 (Schuldiner et al 2008, Simpson et al 2010).

\subsubsection{The conserved Get3 ATPase shuttles TA-proteins}

The conserved cytosolic Get3 ATPase in Saccharomyces cerevisiae (TRC40 in mammalian systems) is the central player of the GET pathway (Figure 1.5 and Figure 1.6). The Get3 dimer shuttles between a cytosolic multiprotein complex that receives the TA precursor proteins from the ribosome, and a transmembrane Get1/Get2 receptor complex at the ERmembrane, where the TA protein precursors are released and integrated into the lipid bilayer (Figure 1.5) (Mariappan et al 2010, Mateja et al 2009, Rome et al 2014, Stefer et al 2011). This cycle is associated with conformational changes in Get3, induced by substrate binding and ATP hydrolysis, allowing Get3 to bind and protect the hydrophobic $\alpha$-helical transmembrane domain (TMD) of TA-proteins (Bozkurt et al 2009, Mateja et al 2015, Mateja et al 2009).

Yeast Get3 is a highly conserved $39 \mathrm{kDa}$ P-loop ATPase and belongs to the signal recognition particle $\mathrm{MinD}$ and BioD (SIMIBI)-type nucleoside triphosphate-binding proteins, comprised of dimeric ATPases as well as 


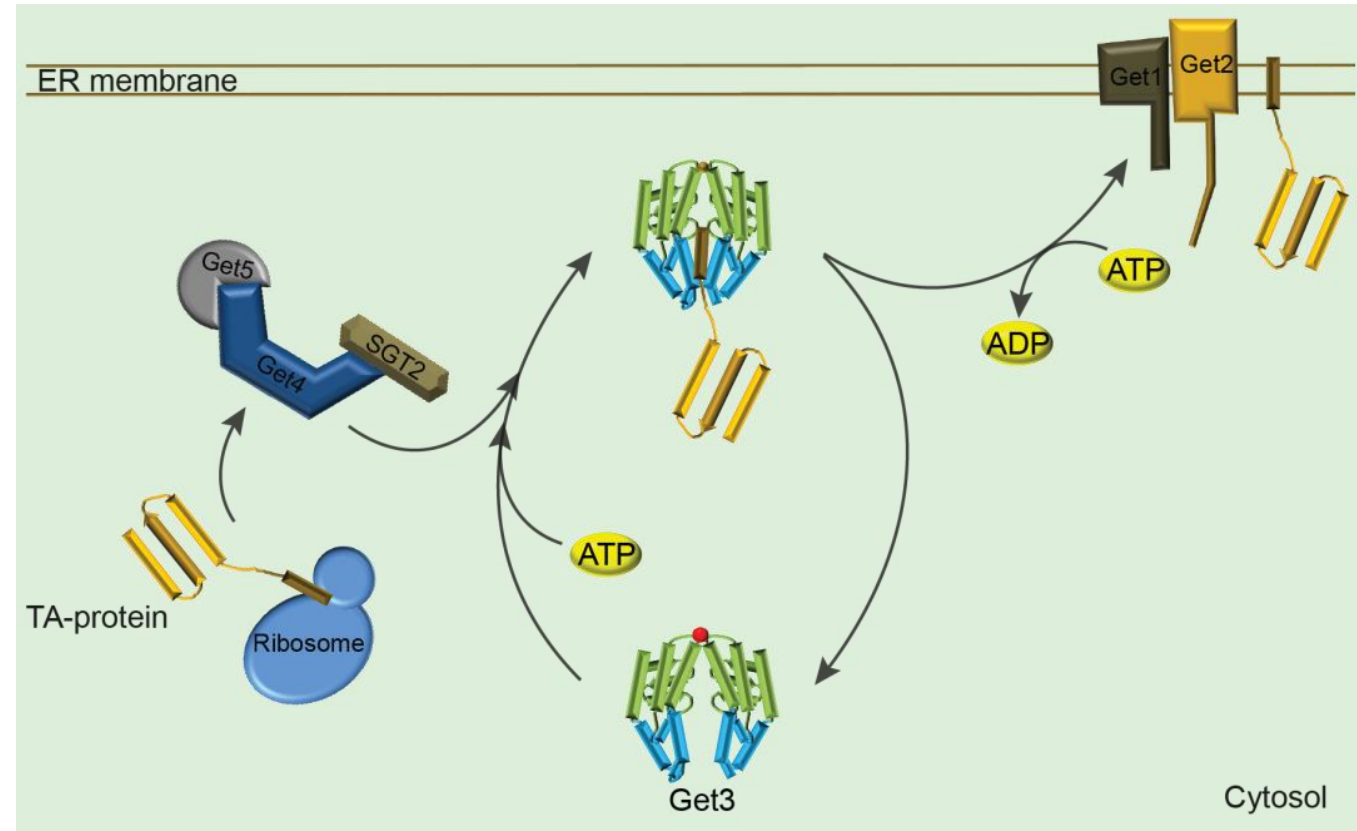

Figure 1. 5: GET pathway mediated TA-protein insertion

Cytosolic Get3 receives tail-anchored (TA) precursor proteins from the ribosome via a multiprotein complex consisting of Sgt2 and Get4/5. Get3 binds the hydrophobic transmembrane domain (TMD, brown) of the TA-protein via its helical subdomain (HSD, blue). ATP binding to Get3's ATPase domain (green) induce s the closed conformation forming a hydrophobic pocket that shields the TMD of the TA-protein. The transmembrane Get1/2 receptors localized in the membrane of the endoplasmic reticulum (ER) membrane recruit Get3 to the ER membrane, stimulate the release of Get3's TA-protein cargo and mediate its insertion into the lipid bilayer of the ER membrane. In this process, Get3 releases ADP, binds ATP and dissociates from the Get $1 / 2$ receptor in its open conformation ready for a new round of TA-shuttling.

GTPases, known as SIMIBI 'twins' (Bange \& Sinning 2013). It has high sequence similarity to the catalytic subunit of $E$. coli arsenical resistance factor ArsA (Figure 1.6) (Shen et al 2003b, Zhou et al 2000). Get3's mammalian homolog TRC40/ASNA1 is closely associated with TA protein biogenesis as well (Colombo et al 2016, Vilardi et al 2014). Up-regulation of ASNA1 has been observed in tuberculosis, breast and ovarian cancers yet the reason for this induction is unknown (Hemmingsson et al 2009a, KurdiHaidar et al 1998, Mistry et al 2007). Importantly, whereas mutations in components of the GET system result in embryonic lethality in higher eukaryotes (Mukhopadhyay et al 2006), yeast cells survive their absence (Metz et al 2006b, Shen et al 2003b). Nevertheless, deletion of the GET3 


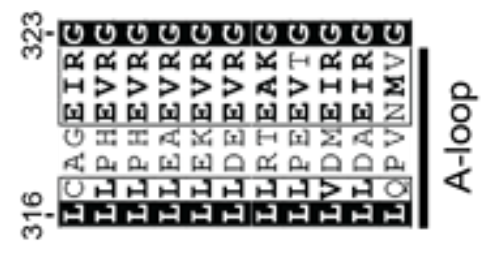

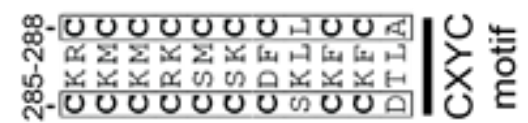
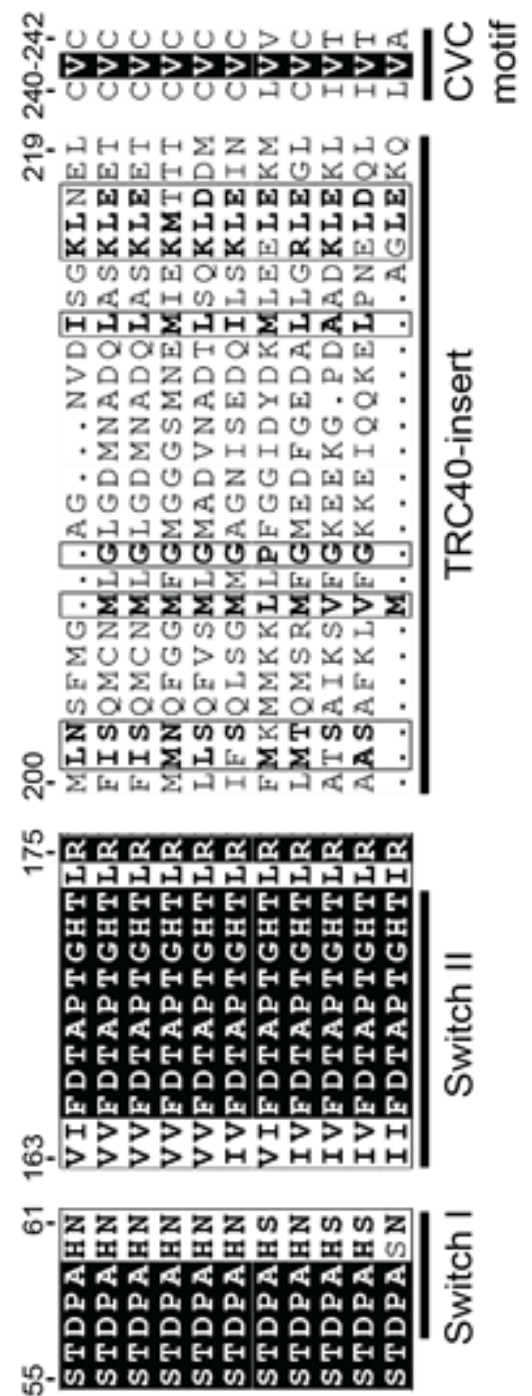

D-UUUUNUAOA

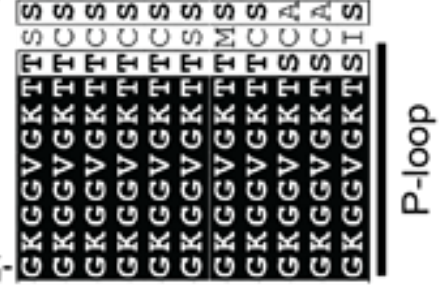

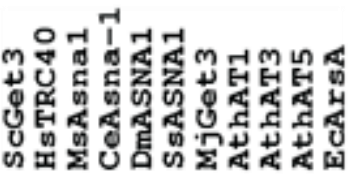

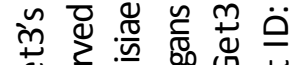

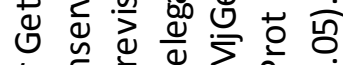

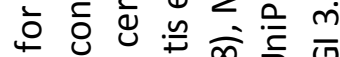

过

类

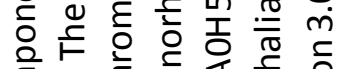

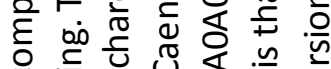

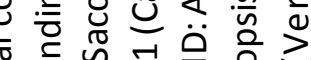

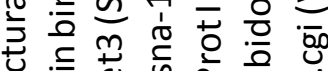

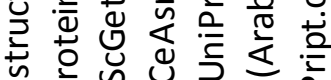

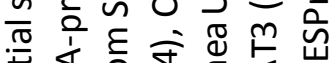

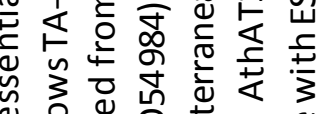

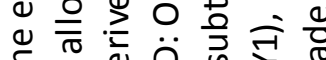

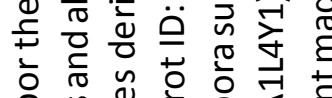

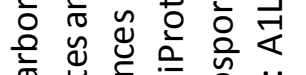

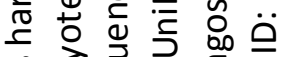

厸密要

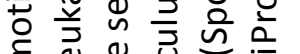

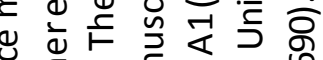

政

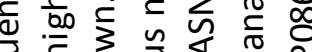

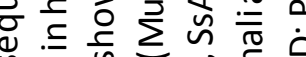

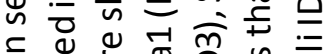

둥

원 क0

흥 흫

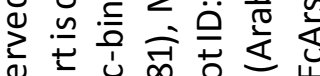

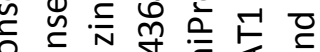

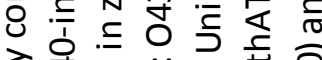

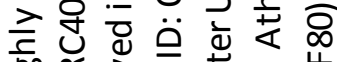

些品 긍ㅎㅇ

\% क

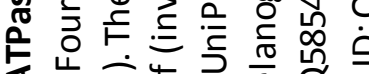

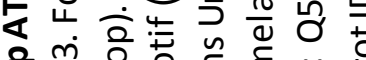

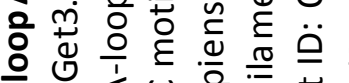

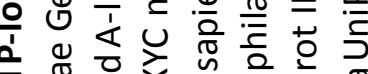

은

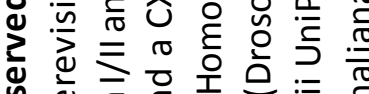

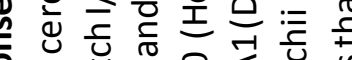

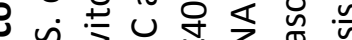

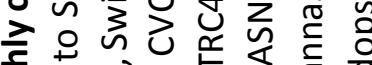

क力

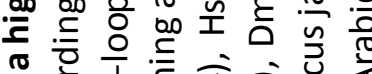

品

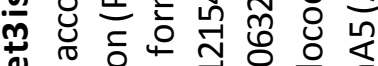

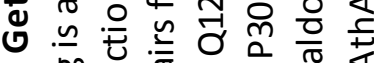
$\ddot{0}$ 年 -

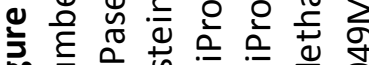

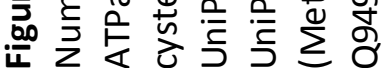


gene in yeast leads to several, seemingly unrelated phenotypes, such as hygromycin sensitivity, copper sensitivity, heat sensitivity and the inability to grow on iron-limiting media (Metz et al 2006b, Schuldiner et al 2008, Shen et al 2003b). However, it is unclear whether these different phenotypes are all related to the reduced integration of specific TA proteins into the ER membrane or are caused by the absence of a potentially second role of Get3 in maintaining metal homeostasis and/or mediating oxidative stress resistance.

Zinc binding stabilizes Get3's dimeric architecture and supports its ATPase function, which directly correlates with its ability to bind TA-protein. Four highly conserved cysteine residues in the Get3 monomer (Cys 240/242/285/288) form a CVC and a CXYC motif (Figure 1.6), the latter one contributes to the stabilization of the Get3 dimer by zinc coordination (Figure 1.7). Mutation of the zinc-coordinating cysteine pair abolishes Get3's dimerization, and fails to rescue the severe growth defect of a get3 deletion strain under various stress conditions (Metz et al 2006b). The zinc ion forms a hinge in dimeric Get3 that enables the open-to-closed transition, which is tightly controlled by ATP binding and hydrolysis. In the nucleotide free state, Get3's ATPase domain (Figure 7, light blue) is in an open conformation (Mateja et al 2009). ATP binding induces the switch to a closed conformation in Get3 ( $\mathrm{Hu}$ et al 2009). ATP hydrolysis and interaction with the Get1/2 receptor releases the TMD of the TA-client into the lipid bilayer of the ER membrane (Figure 1.5) (Rome et al 2013, Sinning et al 2011, Stefer et al 2011). The $\alpha$-helical subdomain (HSD, blue) of Get3 is enriched in hydrophobic amino acids and characterized by an unusually high frequency of methionine residues (Mateja et al 2009). These residues are shielded in the open conformation. Yet, upon ATP binding to the ATPase domain, these residues form a large hydrophobic pocket, which accommodates the hydrophobic TMD of TA-proteins (Mateja et al 2015). A TRC40-insert in Get3's HSD, which is conserved in all eukaryotic Get3 homologues (Figure 1.6), forms a lid that shields the TMD in the hydrophobic pocket (Mateja et al 2009). 


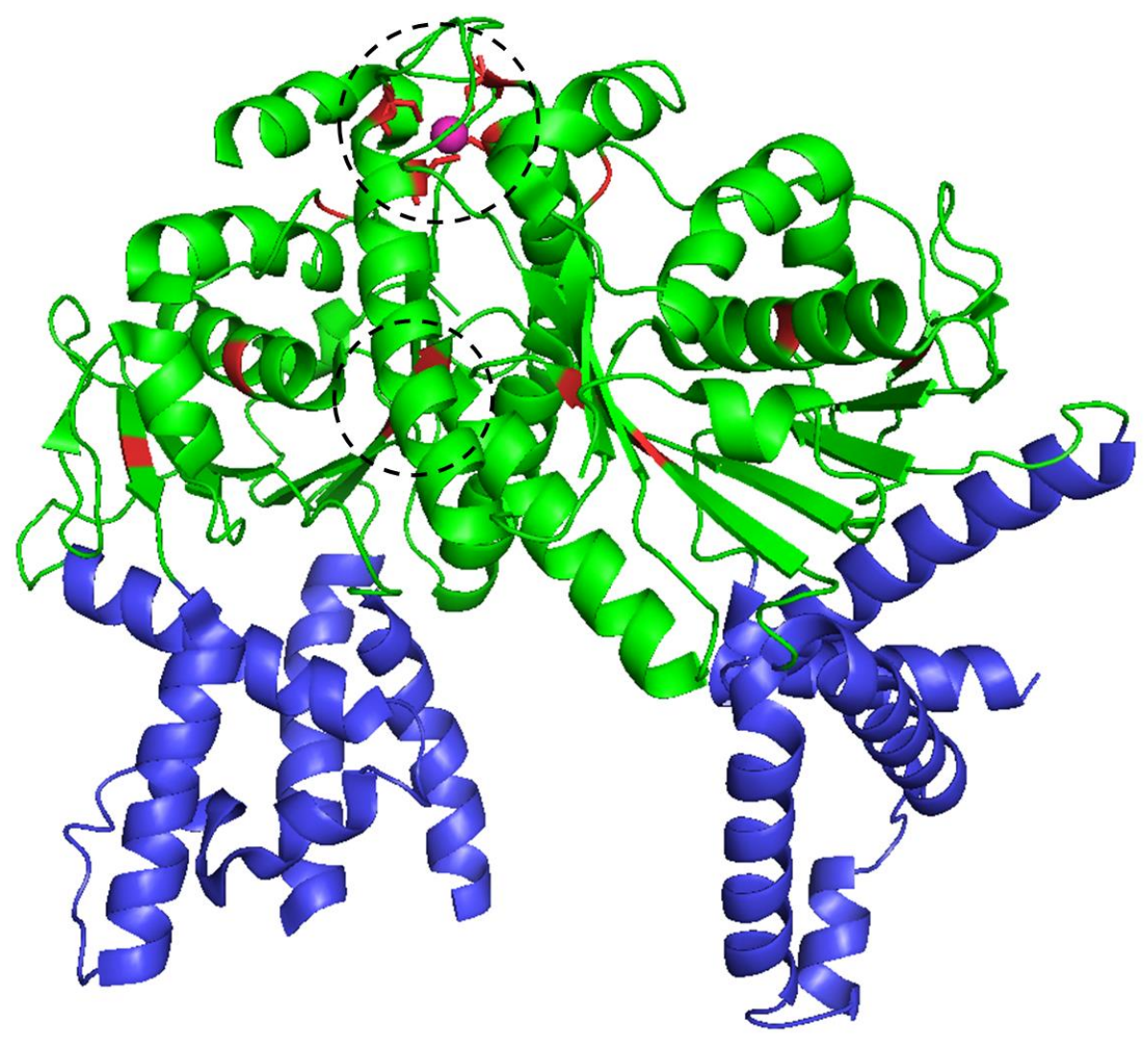

Figure 1. 7 Structure of S. cerevisiae Get3 dimer

Crystal structure of S. cerevisiae Get3 dimer in a nucleotide free state is shown (Hu et al., 2009) (Protein Data Bank ID: 3H84). Get3's ATPase domain is colored in green and the in hydrophobicamino acids enriched helical subdomain is shown in blue. Cysteine residues are shown in red. A zincion (magenta) is coordinated by the CXYC motif (upper dashed circle), whereas the CVC motif is in close vicinity to the ATP-binding site (lower dashed circle). Two conserved cysteine pairs are present in each Get3 monomer. The molecular surface drawing of the structure was mad by Pymol (The PyMOL Molecular Graphics System, Version 1.5.0.4Schrödinger, LLC).

\subsubsection{Get3 exhibits a general in vitro and in vivo chaperone function}

The cysteine arrangement in Get3 is highly reminiscent of the oxidation sensitive zinc-binding motif found in Hsp33. Moreover, aerobically purified Get3 was found to function as an ATP-independent general chaperone in vitro, protecting unfolding proteins against aggregation in a manner akin to oxidized, chaperone-active Hsp33 (Powis et al 2013b). Finally, in vivo 
studies demonstrated that Get3 co-localizes with unfolding proteins and chaperones of the Hsp70 and Hsp100 family in distinct foci during ATPdepleting stress conditions in yeast (Powis et al 2013b). In light of these results, previous studies that showed an unexpected sensitivity of a get3 deletion strain towards various stresses, like copper or heat (Schuldiner et al 2008, Shen et al 2003b) required reassessment, since they suggested that Get3 might exert a second function as general chaperone, and might be the long-sought redox regulated chaperone of eukaryotic cells.

\subsection{Objective}

Redox-sensing and redox-regulation are crucial features that allow cells to respond and adapt to environmental changes. Aim of this thesis was to investigate whether yeast Get3 serves as a redox-regulated chaperone in eukaryotes. This potentially dual function would make Get3 an excellent model protein to answer fundamental questions regarding redox regulation and chaperone function in higher eukaryotes. To answer these questions, we utilized the knowledge and techniques established for the characterization of the redox-regulated chaperone Hsp33. The first question to address was whether Get3 utilizes its conserved zinc-coordinating cysteine motif to sense oxidants similar to Hsp33. Since cysteine oxidation in Hsp33 is known to induce massive structural rearrangements, we investigated how cysteine oxidation impacts Get3's structure and function and to what extent this mechanism is reversible. The second question that we wanted to address concerned the in vivo role of Get3, which required us to separate TA-protein targeting from general chaperone function. Finally, we investigated Get3's crosstalk to other chaperones in the cell. By investigating these aspects, we gained valuable information about the role of Get3's chaperone function in the cell, and uncovered a potential highly dynamic redox-regulated chaperone networks in eukaryotic cells. 


\section{Material and Methods}

All chemicals used in this study for buffer preparation are obtained from Thermo Scientific, Invitrogen, MP Biomedicals, Roche or Sigma Aldrich. Vendors for all other chemicals, enzymes or equipment are marked in the text.

\subsection{Strains and Plasmids}

\subsubsection{E. coli and S. cerevisiae (yeast) strains}

\begin{tabular}{|c|c|c|c|}
\hline Strain & Genotype & Background & Ref. $^{*}$ \\
\hline $\begin{array}{l}\text { E. coli BI21 } \\
\text { DE3 }\end{array}$ & $\begin{array}{l}\text { F- ompT gal dcm lon } \\
\text { hsdSB(rB- mB-) } \lambda(D E 3 \text { [lacl } \\
\text { lacUV5- } 7 \text { gene } 1 \text { ind1 sam7 } \\
\text { nin5] }\end{array}$ & & Novagen \\
\hline BY4741 & $\begin{array}{l}\text { MATa his3 } 31 \text { leu2 } \Delta 0 \\
\text { met15 } 10 \text { ura3 } \Delta 0\end{array}$ & S288C & 1 \\
\hline get3 & $\begin{array}{l}\text { MATa his3 } \Delta 1 \text { leu2 } \Delta 0 \\
\text { met15 } \Delta 0 \text { ura3 } \Delta 0 \\
\text { ydl100c::KanR }\end{array}$ & BY4741 & 2 \\
\hline get1/get2/get3 & $\begin{array}{l}\text { MATa his } 3 \Delta 1 \text { leu2 } \Delta 0 \\
\text { met1 } 5 \Delta 0 \text { ura3 } \Delta 0 \text { get1::KanR, } \\
\text { get2::NatR, get3::PhleoR }\end{array}$ & BY4741 & 3 \\
\hline
\end{tabular}

\subsubsection{Plasmids}

\begin{tabular}{|c|c|c|c|c|}
\hline Name & Description & $\begin{array}{l}\text { Marker } \\
\text { (Yeast) }\end{array}$ & $\begin{array}{l}\text { Marker } \\
\text { (E. coll) }\end{array}$ & Ref. $^{*}$ \\
\hline pJ488 & pQE80-10His-ZZ-Get3 & & Amp & 4 \\
\hline pAA1349 & pQE80-10His-ZZ-Get3D57E & & Amp & 5 \\
\hline pAD1474 & pet280 Get3/193D & & Kan & 6 \\
\hline pG307 & p416Met25-Get3WT & Ura & Amp & 5 \\
\hline pG309 & p416Met25-Get3D57E & Ura & Amp & 5 \\
\hline ppG310 & p416Met25-GFP-Get3WT & Ura & Amp & 5 \\
\hline pG308 & p416Met25-GFP-Get3D57E & Ura & Amp & 5 \\
\hline pAA1307 & p415Met25-mCherry-Sed5 & Leu & Amp & 5 \\
\hline pX1157 & p413Met25-GFP-Sed5 & His & Amp & 5 \\
\hline pAF1558 & p416Met25-Get3 ${ }^{1193 D}$ & Ura & Amp & Vilardi F. \\
\hline pAF1559 & p416Met25-GFP-Get3 ${ }^{1193 D}$ & Ura & Amp & Vilardi F. \\
\hline
\end{tabular}

${ }^{*}$ 1(Brachmann et al 1998); 2(Jonikas et al 2009); 3(Schuldiner et al 2008); 4(Metz 
et al 2006b); 5(Powis et al 2013b); 6(Mateja et al 2009)

\subsection{Molecular methods}

\subsubsection{Plasmid construction}

For p416Met25-Get3 ${ }^{1193 D}$, the coding sequence of Get3 ${ }^{1193 D}$ was amplified by PCR from pLAC33-Get31193D (Mateja et al 2009) using the primers 5''TATGATACTAGTATGGATTTAACCGTGGAA-3' and 5'- ATCATACTCGAGCTATTCCTTATCTTCTAA-3' containing Spel and Xhol restriction sites, respectively. For p416Met25-GFP-Get31193D, a Spel/BamHI fragment was originated from p416Met25-Get3 ${ }^{1193 D}$ and ligated to p416Met25-GFPGet $^{\mathrm{WT}}$ previously digested with the same restriction endonucleases.

\subsubsection{Polymerase chain reaction (PCR)}

PCR was carried out to amplify DNA fragments encoding protein sequences of interest. $100 \mathrm{ng}$ template DNA were mixed with $200 \mu \mathrm{M}$ deoxynucleotides (dNTPs, NEB), $1 \mu \mathrm{M}$ oligonucleotide primers, 2 units of phusion DNA polymerase (NEB) in $50 \mu \mathrm{l}$ of 1 -fold phusion high fidelity buffer (NEB). The reaction mixture was then transferred to a thermal cycler (Veriti) and PCR reaction was carried out with the following program:

1) Initial denaturation at $98^{\circ} \mathrm{C}$ for $30 \mathrm{sec}$

2) Melting temperature at $90^{\circ} \mathrm{C}$ for $10 \mathrm{sec}$

3) Annealing temperature* at $50^{\circ} \mathrm{C}$ for $30 \mathrm{sec}$

4) Polymerization at $72^{\circ} \mathrm{C}$ for $45 \mathrm{sec}$

$>$ Repeat step 2 to 5 for 30 cycles

5) Final Extension at $72^{\circ} \mathrm{C}$ for $5 \mathrm{~min}$

6) Hold at $4^{\circ} \mathrm{C}$

${ }^{*}$ Adjust annealing temperature to primer with the lowest melting temperature

DNA fragments generated by PCR were purified using a PCR product purification kit (Roche) according to the manufacturer's instructions. 


\subsubsection{DNA digest and ligation}

Plasmid DNA or DNA fragments were digested with one or multiple restriction enzymes (Fermentas). Respectively, $2 \mu \mathrm{g}$ plasmid DNA or 6-8 $\mu \mathrm{g}$ DNA fragments were incubated with 2 or 6-8 units of restriction enzyme in buffer according to the manufacturer's instructions. The reaction mix was incubated for at least $60 \mathrm{~min}$ at $37^{\circ} \mathrm{C}$. Subsequently, 1 unit of calf intestinal alkaline phosphatase (CIAP, NEB) was added to the reaction mix and the incubation at $37^{\circ} \mathrm{C}$ was continued for $30 \mathrm{~min}$. Digested DNA fragments and linearized plasmid DNA were separated by agarose gel electrophoresis and purified subsequently.

To ligate the digested DNA fragment and linearized plasmid, $100 \mathrm{ng}$ of linearized plasmid and a 5-fold molar excess of the fragment were supplemented with 1 unit of T4-DNA ligase (NEB) and 1-fold ligation buffer (NEB) in a volume of $30 \mu \mathrm{l}$. The reaction mixture was incubated for $16 \mathrm{~h}$ at $18^{\circ} \mathrm{C}$ and for $10 \mathrm{~min}$ at $65^{\circ} \mathrm{C}$ to heat inactivated the ligase.

\subsubsection{Agarose gel electrophoresis}

Digested DNA fragments or linearized plasmids DNA were analyzed or purified via agarose gel electrophoresis. $1 \% \quad(\mathrm{w} / \mathrm{v})$ agarose (MP Biomedicals) was dissolved in $150 \mathrm{ml}$ Tris-acetate-EDTA (TAE) buffer (40 mM Tris, $20 \mathrm{mM}$ acetic acid, $1 \mathrm{mM}$ EDTA pH 8.0) at $95^{\circ} \mathrm{C}$ for $3 \mathrm{~min}$, $10 \mu \mathrm{l}$ SafeView (Applied Biological Materials) were added and the solution was kept for $45 \mathrm{~min}$ at room temperature (RT) to solidify. DNA samples were mixed with 1-fold gel loading dye (Fisher) and loaded onto the agarose gel. Agarose gel electrophoresis was performed by following the manufacturer's specifications (BioRad) in TAE buffer. Subsequently, the gels were analyzed under ultra violet (UV) light at $365 \mathrm{~nm}$ and bands containing DNA fragments were excised. DNA-containing agarose pieces were purified using a PCR product purification kit (Roche) according to the manufacturer's instructions. DNA concentrations were determined spectroscopically using a NanoDrop 2000 UV-Vis Spectrophotometer. 


\subsubsection{Transformation of $E$. coli for storage or DNA amplification}

Plasmid DNA encoding protein of interest was transformed into electrocompetent DH5alpha, or electrocompetent BL21 Rosetta2(DE3)/pLysS (Novagen) E.coli cells for plasmid DNA amplification or for protein expression, respectively. Briefly, to generate electrocompetent cells, $100 \mu \mathrm{l}$ of an overnight culture containing either DH5alpha or BL21 cells were diluted into $5 \mathrm{ml}$ fresh LB-media (10 g/l tryptone, $5 \mathrm{~g} / \mathrm{l}$ yeast extract, $0.5 \mathrm{~g} /(\mathrm{NaCl})$ and incubated at $37^{\circ} \mathrm{C}$ under constant agitation to mid-log phase $(\sim 2 \mathrm{~h})$. Then, $1 \mathrm{ml}$ aliquots were pelleted $\left(16,000 \times \mathrm{g}, 4^{\circ} \mathrm{C}\right)$ for $1 \mathrm{~min}$ and resuspended in $1 \mathrm{ml}$ ice-cold distilled water. Subsequently, this procedure was repeated for a total of three rinse cycles. The final cell pellet was resuspended in $\sim 100 \mu \mathrm{l}$ of ice-cold water on ice, the plasmid DNA was added and the mixture was transferred into a precooled electroporation cuvette. The electroporation was carried out in a Gene Pulser electroporation unit (BioRad) according to the manufacturer's instructions. Cells were then recovered from the electroporation cuvette, dissolved in $1 \mathrm{ml}$ of $\mathrm{SOC}$ medium ( $20 \mathrm{~g} / \mathrm{l}$ tryptone, $5 \mathrm{~g} / \mathrm{l}$ yeast extract, $0.5 \mathrm{~g} / \mathrm{l} \mathrm{NaCl}$, $2.5 \mathrm{mM} \mathrm{KCl}, 10 \mathrm{mM} \mathrm{MgCl}$, $20 \mathrm{mM}$ glucose), incubated at $37^{\circ} \mathrm{C}$ for $60 \mathrm{~min}$ and plated onto LB agar plates (10 g/l tryptone, $5 \mathrm{~g} / \mathrm{l}$ yeast extract, $0.5 \mathrm{~g} / \mathrm{l} \mathrm{NaCl}, 15 \mathrm{~g} / \mathrm{l}$ agar), containing selective antibiotics.

LB-medium containing selective antibiotic was inoculated with single colonies of the respective transformed E.coli cells and cultured at $37^{\circ} \mathrm{C}$ overnight $(\sim 16 \mathrm{~h})$. For a permanent glycerol stock, $0.5 \mathrm{ml}$ of the culture was transferred to a microfuge tube containing $60 \%$ glycerol and frozen at $-80^{\circ} \mathrm{C}$. For plasmid DNA purification, cells were harvested by centrifugation and plasmid DNA was extracted with either the NucleoSpin Plasmid kit for a small scale plasmid purification or with the NucleoBond Xtra Midi (Macherey-Nagel) for large scale plasmid purifications according to the manufacturer's instructions. 


\subsection{Protein biochemistry}

\subsubsection{Protein SDS-PAGE}

To separate proteins by sodium dodecyl sulfate-polyacrylamid gel electrophoresis (SDS-PAGE), pre-casted $12 \%$ gels were used (TGX-gel, BioRad). Alternatively, custom-made $12 \%$ SDS-polyacrylamid gels were prepared. Therefore, a separation $(8.0 \mathrm{ml} 30 \%$ Acrylamide $(\mathrm{w} / \mathrm{v}), 5 \mathrm{ml} 1.5 \mathrm{M}$ Tris $\mathrm{pH} 8.8,0.2 \mathrm{ml} 10 \% \operatorname{SDS}(\mathrm{w} / \mathrm{v}), 0.2 \mathrm{ml}$ ammonium persulfate, $6.6 \mathrm{ml}$ $\left.\mathrm{H}_{2} \mathrm{O}, 12 \mu \mathrm{l} \mathrm{TEMED}\right)$ and a stacking gel $(1.7 \mathrm{ml} 30 \%$ Acrylamide (w/v), $5 \mathrm{ml}$ $1 \mathrm{M}$ Tris $\mathrm{pH} 6.8,0.1 \mathrm{ml} \mathrm{10 \%} \mathrm{SDS} \mathrm{(w/v),} 0.1 \mathrm{ml}$ ammonium persulfate, $10 \mu \mathrm{l}$ TEMED) were poured. Protein samples were supplemented with $1 / 5$ of their volume of $5 x$ Laemmli buffer (300 mM Tris, $60 \%$ glycerol, $10 \%$ SDS, $0.05 \%$ bromphenol blue). Optionally, $125 \mathrm{mM} \beta$-mercaptoethanol was added to establish reducing conditions. Pelleted samples were supplemented analogously with $1 \times$ Laemmli buffer (60 mM Tris, $12.5 \%$ glycerol, $2 \%$ SDS, $0.01 \%$ bromphenol blue, $125 \mathrm{mM} \beta$-mercaptoethanol). Samples dissolved in reducing buffer were boiled at $95^{\circ} \mathrm{C}$ for $5 \mathrm{~min}$; nonreduced samples were directly loaded onto the SDS-PAGE. Gel electrophoresis was performed by following the manufacturer's specifications (BioRad) in SDS running buffer (25 mM Tris, $192 \mathrm{mM}$ glycine, 0.1\% SDS, pH 8.0). Proteins were visualized by Coomassie blue (Wong et al 2000) or silver staining (Nesterenko et al 1994).

\subsubsection{Western blotting}

Proteins separated by SDS-PAGE were then blotted onto a nitrocellulose membrane (GE Healtcare) using a wet blotting TE22 Mighty Small Transfer Tank (GE Healtcare) filled with transfer buffer (25 mM Tris, $192 \mathrm{mM}$ Glycine, $\mathrm{pH}$ 8.3) following the manufacturer's specifications. The membrane was stained with Ponceau to visualize the protein bands. Subsequently, membranes were incubated with $5 \%$ milk powder (w/v) dissolved in TBS (25 mM Tris* $\mathrm{HCl} \mathrm{pH} \mathrm{7.4,} 135 \mathrm{mM} \mathrm{NaCl}, 3 \mathrm{mM} \mathrm{KCl}, 0.02 \% \mathrm{NP}-40$ ) buffer for $1 \mathrm{~h}$ to block free binding sites. Primary mouse monoclonal antibody against the cytosolic enzyme Pgk1 (Molecular Probes) was used as a loading control 
or a guinea pig serum against Get3 (Metz et al 2006a) diluted 1:1,000 in $5 \%$ TBS-milk were added and incubated under agitation for $3 \mathrm{~h}$ at room temperature (RT). The membrane was briefly washed with TBS buffer (3x5 min), and incubated with the secondary anti-mouse (Li-COR, 680RD) or anti-guinea pig (Li-COR, $800 \mathrm{CW}$ ) antibody diluted $1: 10,000$ in $5 \%$ TBSmilk for $2 \mathrm{~h}$. Detection of the secondary antibody was carried out with an Odyssey LiCOR (LiCOR, Germany) imaging system according to the manufacturer's specifications.

\subsubsection{Spin-down assay}

To separate the soluble and the insoluble aggregated fraction in a sample, the aggregated insoluble protein was pelleted by centrifugation (30 min, RT, $16,000 \times \mathrm{g}$ ). Subsequently, the soluble fraction in the supernatant (SN) was separated from the insoluble fraction in the pellet $(P)$. Both fractions were filled up to the same volume with a final concentration of $1 x$ Laemmli buffer and the pellet was dissolved by vortexing. Then, the fractions were analyzed on reducing SDS-PAGE as previously described. Soluble samples with low protein concentrations were TCA precipitated to adjust the concentrate prior to SDS-PAGE.

\subsubsection{Trichloroacetic acid (TCA)-precipitation}

For TCA precipitation of proteins, the final TCA concentration in the sample was adjusted to $10 \%(\mathrm{v} / \mathrm{v})$ by adding ice cold $50 \% \mathrm{v} / \mathrm{v}$ TCA to the sample. The samples were vortexed and incubated for $30 \mathrm{~min}$ on ice. Supernatant and the pellet containing the precipitated proteins were separated by centrifugation ( $30 \mathrm{~min}, 4^{\circ} \mathrm{C}, 16,000 \times \mathrm{g}$ ). The pellet was washed twice with $200 \mu \mathrm{l}$ pure ice cold acetone, air dried at room temperature and processed as described above.

\subsection{Protein purification}

\subsubsection{Purification of wild-type Get3 and Get3 mutant variants}

Get3 wild-type (wt) and the D57E mutant variant were expressed in E. coli 
BL21 Rosetta2(DE3)/pLysS from a pQE80 derivative as a fusion of two $Z$ domains (IgG-binding domain of protein A) to Get3 (Metz et al 2006b) and an $\mathrm{N}$-terminal $6 \times \mathrm{His}$ tag in front of the $\mathrm{Z}$ domain. In addition, the vector contains a tobacco etch virus (TEV) protease cleavage site between the $Z$ domain and the polylinker. The Get3 1193D mutant variant was expressed from a pet280 vector (kind gift of Robert Keenan) containing a TEV protease cleavage site between the $\mathrm{N}$-terminal $6 \times$ His tag and a polylinker. Overnight cultures of cells were diluted 1:100 into standard LB media, after cells reached an $A 600$ of $\sim 0.7$, protein expression was induced with $0.4 \mathrm{mM} \mathrm{IPTG}$ and cells were cultivated for another $4 \mathrm{~h}$ at $30^{\circ} \mathrm{C}$. Then the cells were pelleted and resuspended in extraction buffer $(50 \mathrm{mM}$ Tris, $50 \mathrm{mM} \mathrm{NaCl}, 2$ $\mathrm{mM} \mathrm{MgAc}, 1 \mathrm{mM}$ imidazole, $2 \mathrm{mM} \mathrm{DTT}, \mathrm{pH}$ 7.5), supplemented with one tablet of complete EDTA-free protease inhibitor (Roche) and $1 \mathrm{mM}$ phenylmethane sulfonyl fluoride (PMSF, Sigma Aldrich). Cells were lysed using a commercial French press (Thermo, $3 \times 1,300$ psi). The cell lysate was cleared by centrifugation $\left(45,000 \times \mathrm{g}, 45 \mathrm{~min}\right.$ at $\left.4^{\circ} \mathrm{C}\right)$, subsequently, the supernatant was filtered (filter membrane pore size of $0.8 \mu \mathrm{M}$, Thermo Scientific) and loaded on a nickel-NTA column (QIAGEN) equilibrated with extraction buffer. The resin was washed respectively with extraction buffer containing $4 \mathrm{mM} \mathrm{MgATP}, 0.5 \mathrm{M} \mathrm{NaCl}$ and extraction buffer alone. Bound protein was eluted with extraction buffer supplemented with $0.5 \mathrm{M}$ imidazole. Fractions containing Get3 were pooled and buffer-exchanged to low-salt buffer A (50 mM Tris, $20 \mathrm{mM} \mathrm{NaCl}, \mathrm{pH}$ 7.5) using Zeba desalting columns (Thermo Scientific). For further purification the protein was loaded on an anion-exchange chromatography column (Q-sepharose HP, GE Healthcare) equilibrated with low-salt buffer $A$. Bound protein was eluted with a $60 \mathrm{ml}$ gradient from low-salt buffer A to high-salt buffer B $(50 \mathrm{mM}$ Tris, $0.5 \mathrm{M} \mathrm{NaCl}, \mathrm{pH}$ 7.5). Get3-containing fractions were pooled and the purified protein was simultaneously cleaved with $6 \times$ His-tagged TEV protease at a TEV:Get3 ratio of $1: 100$ and dialyzed for $20 \mathrm{~h}$ at $4^{\circ} \mathrm{C}$ against cleavage buffer (50 mM Tris, $50 \mathrm{mM} \mathrm{NaCl}, \mathrm{pH}$ 7.5) containing $0.5 \mathrm{mM} \mathrm{DTT}$ and $0.5 \mathrm{mM}$ EDTA. Uncleaved Get3, the His-tagged Z domain and the $6 \times$ Histagged TEV protease were removed by subsequent passage over a nickelNTA column. Purified Get3 was concentrated to $100 \mu \mathrm{M}$ and stored in 
$50 \mathrm{mM}$ Tris, $50 \mathrm{mM} \mathrm{NaCl}, 2 \mathrm{mM} \mathrm{MgAc}, \mathrm{pH} 7.5$ at $-80^{\circ} \mathrm{C}$. The efficiency of cleavage was quantified by SDS-PAGE and was $>95 \%$. Get3 concentrations were determined spectroscopically using a Jasco spectrophotometer V-550.

\subsubsection{Determination of protein concentration}

Get3 concentrations were determined spectroscopically using a Jasco spectrophotometer V-550. The acquired spectra was buffer-corrected and the extinction coefficient of $\varepsilon 280 \mathrm{~nm}=19,940 \mathrm{M}^{-1} \mathrm{~cm}^{-1}$ was used to calculate the concentration of wt Get3t and mutant variants. Unless otherwise mentioned, wt Get3 and the mutant variants were treated in the same way for all subsequent measurements and are collectively referred to as Get3.

\subsubsection{Proteins used during this study}

TEV protease purified by Ken Wan for lab use was stored at $-80^{\circ} \mathrm{C}$. Bacterial DnaK, DnaJ and GrpE were previously purified by Claudia Cremers and Filipa Teixeira and stored in a lab stock at $-80^{\circ} \mathrm{C}$. Yeast Ydj1 and human HSPA1A, stored at $-80^{\circ} \mathrm{C}$, were a kind gift of Eric Tse, University of Michigan, Ann Arbor, Southworth Lab.

\subsection{Preparation of modified Get3}

\subsubsection{Reduction and oxidation of Get3}

To obtain a homogeneous stock solution of reduced or oxidized Get3, purified Get3 in storage buffer ( $50 \mathrm{mM}$ Tris, $50 \mathrm{mM} \mathrm{NaCl}, 5 \mathrm{mM} \mathrm{MgAc}$, $\mathrm{pH}$ 7.5) was supplemented with $5 \mathrm{mM}$ dithiothreitol (DTT) and incubated for $1 \mathrm{~h}$ at $30^{\circ} \mathrm{C}$ and mild shaking (300 rpm). Later, to obtain "fully" reduced and chaperone-inactive protein, purified Get3 was diluted down to $5 \mu \mathrm{M}$ in

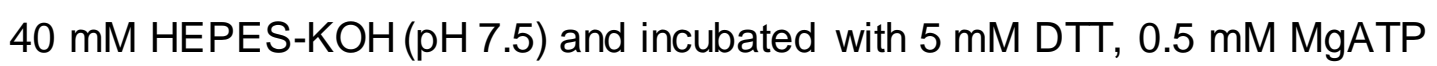
and $5 \mu \mathrm{M} \mathrm{ZnCl} 2$ for $6 \mathrm{~h}$ at $30^{\circ} \mathrm{C}$, shaking (300 rpm). Subsequently, the protein was dia-filtrated using a amicon centrifugal filter unit (EMD Millipore) with $40 \mathrm{mM}$ HEPES-KOH (pH 7.5), therefore the protein was concentrated and diluted 1:10 in HEPES-KOH buffer for at least two consecutive times, to dilute out the MgATP. All samples were applied to a Zeba spin column 
(Thermo Scientific) or Micro Spin 30 (Bio-Rad) column equilibrated with $40 \mathrm{mM}$ HEPES-KOH (pH 7.5) to remove the reductants. Reduced Get3 was either directly used for further assays, stored at $-80^{\circ} \mathrm{C}$, or oxidized.

For oxidation, Get3red was incubated with either $2 \mathrm{mM} \mathrm{H}_{2} \mathrm{O}_{2} / 50 \mu \mathrm{M} \mathrm{CuCl} 2$ for 4 or $10 \mathrm{~min}$ (maximum $240 \mathrm{~min}$ ), or with various concentrations of $\mathrm{CuCl}_{2}$ between mixing time and $240 \mathrm{~min}$ (as indicated in the figure legend), or with $2 \mathrm{mM} \mathrm{H} 2 \mathrm{O}_{2}$ for a maximum of $240 \mathrm{~min}$ at $37^{\circ} \mathrm{C}$ and mild shaking (300 rpm). At indicated time points the protein was tested directly for chaperone activity (stated in the figure legend) e.g. activation kinetics shown in Figure 3.1.3. For the majority of the subsequent assays, Get3red was oxidized with $2 \mathrm{mM}$ $\mathrm{H}_{2} \mathrm{O}_{2} / 50 \mu \mathrm{M} \mathrm{CuCl}_{2}$ for $10 \mathrm{~min}$ and the oxidants were removed as described above for the removal of the reductant. To remove copper in the experiment shown in Figure 3.1.4, the protein was incubated with $5 \mathrm{mM}$ TPEN (10 min at $30^{\circ} \mathrm{C}$ ) before loading it onto the spin column. Oxidized Get3 was either used for further assays or stored at $-80^{\circ} \mathrm{C}$. When needed, Get3 was concentrated using amicon centrifugal filter units with a molecular cut-off of $30 \mathrm{kDa}$ (EMD Millipore).

\subsubsection{Inactivation of the Get3ox chaperone}

For the inactivation experiments Get3 was oxidized for 4 min ( $2 \mathrm{mM} \mathrm{H}_{2} \mathrm{O}_{2} / 50 \mu \mathrm{M} \mathrm{CuCl}_{2}$ ), the oxidants were removed and Get3ox was diluted to a concentration of $5 \mu \mathrm{M}$ in $40 \mathrm{mM}$ HEPES-KOH (pH 7.5). Subsequently, the protein was incubated with $5 \mathrm{mM}$ DTT for up to $6 \mathrm{~h}$ at $30^{\circ} \mathrm{C}$, shaking (300 rpm) in the absence or presence of $2 \mathrm{mM} \mathrm{MgATP}$ and $5 \mu \mathrm{M} \mathrm{ZnCl}$. The re-reduced and inactivated protein was either directly used for chaperone activity measurements or buffer exchanged to remove the reductants and the other components as described above (stated in the figure legend). 


\subsection{Activity Measurements of Get3}

\subsubsection{Chaperone activity measurements}

To analyze the chaperone activity, the influence of Get3 on the aggregation of thermally unfolding luciferase (Promega) or chemically denatured citrate synthase (Sigma Aldrich) was tested. $12 \mu \mathrm{M}$ citrate synthase (CS) or alternatively luciferase were denatured in $40 \mathrm{mM}$ HEPES, $6 \mathrm{M}$ guanidine hydrochloride (Gdn-HCl), pH7.5 overnight at RT. To initiate protein aggregation, chemically denatured CS or luciferase were diluted to a final concentration of $0.075 \mu \mathrm{M}$ into $40 \mathrm{mM}$ HEPES, $\mathrm{pH} 7.5$ at $30^{\circ} \mathrm{C}$ under continuous stirring. The maximum light scattering signal was reached after $240 \mathrm{sec}$ of CS incubation and was set to $0 \%$ chaperone activity. To determine the effect of Get3 on the CS or luciferase aggregation, Get3 was diluted at various concentrations (indicated in the figure legends) into the buffer and light scattering was monitored after addition of chemically denatured substrate. For thermal unfolding measurements $12 \mu \mathrm{M}$ luciferase was freshly prepared in $40 \mathrm{mM}$ MOPS, $50 \mathrm{mM} \mathrm{KCl}, \mathrm{pH} 7.5$ and diluted to a final concentration of $0.1 \mu \mathrm{M}$ into $40 \mathrm{mM}$ MOPS, $50 \mathrm{mM} \mathrm{KCl}, \mathrm{pH} 7.5$ at $43^{\circ} \mathrm{C}$ to initiate protein aggregation. Where indicated a combination of $5 \mathrm{mM} \mathrm{DTT}$, $2 \mathrm{mM} \mathrm{MgATP}$ and $5 \mu \mathrm{M} \mathrm{ZnCl} 2$ was added after $\sim 900 \mathrm{sec}$ of aggregation. Light scattering signal for all aggregation measurements was monitored in a Hitachi F4500 fluorescence spectrophotometer equipped with a temperature-controlled cuvette holder and stirrer. $\lambda_{\mathrm{em}} / \lambda_{\mathrm{ex}}$ were set to $360 \mathrm{~nm}$, excitation/ emission slit widths were set to $2.5 \mathrm{~nm}$ and the photomultiplier (PMT) voltage was set to $700 \mathrm{~V}$.

\subsubsection{Standard curve for Get3's chaperone activity}

To determine the linear range of the chaperone assays I analyzed the influence of increasing amounts of fully oxidized Get3 on the light scattering signal of CS (Figure 2.1). This was essential to interpret the activation and inactivation of Get3 kinetically. The influence of Get3ox on CS light scattering 


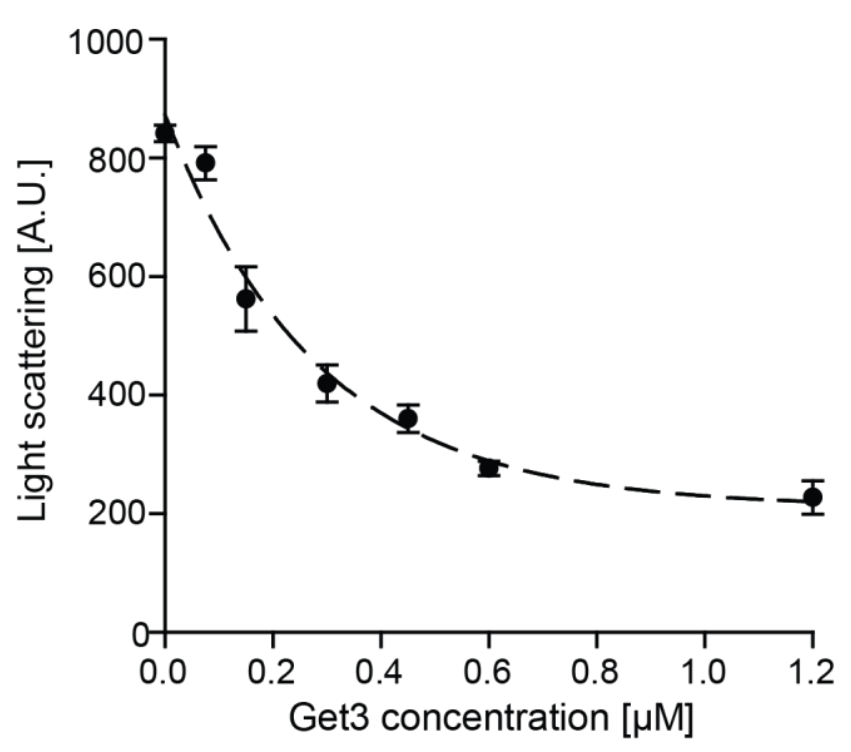

Figure 2. 1: Standard curve for the chaperone activity of Get3 ${ }_{\mathrm{ox}}$

Light scattering of $75 \mathrm{nM}$ chemically denatured citrate synthase (CS) upon dilution into buffer in the absence or presence of increasing amounts of oxidized Get3 was measured. The light scattering signals after $240 \mathrm{sec}$ were plotted against the concentration of Get3ox (solid circle) and the exponential decay was fitted (dashed line). The parameters were used to determine the percent activity of Get3 in subsequent experiments.

followed an exponential decay, which was fitted according to equation 1:

$$
f=210.95+661.81 \times \mathrm{e}^{(-3.58 * \mathrm{x})}
$$

with $f$ being the measured light scattering signal and $x$ reflecting the concentration of active Get3. In case not stated differently in the figure legend, the same parameters were used to determine the percent activity for Get3 in subsequent experiments.

\subsubsection{Activation and inactivation kinetics of Get3}

Get3 was incubated with the respective oxidants or reductants as described previously. To monitor the activation and inactivation kinetics, at defined time points, aliquots were taken and diluted to a final concentration $0.3 \mu \mathrm{M}$ Get3 (MW $=0.01 \mathrm{mg} / \mathrm{ml}$ ) into assay buffer, which corresponds to a $4: 1$ molar excess of Get3 to CS (MW =0.004 mg/ml). Chaperone activity measurements were monitored as described. The maximum of the light scattering signal reached with $0.3 \mu \mathrm{M}$ Get3 was calculated using 
equation 1. Activation and inactivation kinetics were performed at least in triplicates (as stated in the figure legend). The data points were fitted (SigmaPlot V13) to an exponential function with a double exponential fit for $\mathrm{H}_{2} \mathrm{O}_{2} / \mathrm{Cu}^{2+}$ and $\mathrm{Cu}^{2+}$ activation, respectively (equation 2), or a single exponential fit for $\mathrm{H}_{2} \mathrm{O}_{2}$ activation using equation 3:

Equation $2 f=\mathrm{y} 0+\mathrm{a} *\left(1-\mathrm{e}^{(-\mathrm{b} * \mathrm{x})}\right)+\mathrm{c} *\left(1-\mathrm{e}^{(-\mathrm{d} * \mathrm{x})}\right)$

Equation $3 \quad f=\mathrm{y} 0+\mathrm{a} *\left(1-\mathrm{e}^{(-\mathrm{b} * \mathrm{x})}\right)$

with $f$ being the relative chaperone activity and $x$ reflecting the time of incubation. $y 0$ is the basal chaperone activity of Get3red, a and c reflect the maximal chaperone activity for the $1^{\text {st }}$ and $2^{\text {nd }}$ phase of the kinetic. $b$ and $d$ are the rate constants for the $1^{\text {st }}$ and $2^{\text {nd }}$ phase of the activation kinetic, respectively. Since equation 3 describes a single exponential fit, it only consists of parameters $\mathrm{y} 0, \mathrm{a}$ and $\mathrm{b}$. The halftimes ( $\mathrm{t} 1 / 2)$ for the activation of Get3 were obtained from the rate constant using equation 4:

$$
t_{1 / 2}=\frac{\ln (2)}{\text { b ord }}
$$

with $b$ and $d$ being the rate constants for the $1^{\text {st }}$ or $2^{\text {nd }}$ phase of the kinetic.

For the inactivation kinetics shown for the chaperone activity in Figure 3.1.10 $\mathrm{B}$ and the relative refolding in Figure 3.1.11 $\mathrm{B}$ the data points were fitted (SigmaPlot V13) to an exponential decay function. A single exponential fit with two parameters was used for the chaperone activity (equation 5) and a single exponential fit with three parameters for the relative refolding according to equation 6 :

\section{Equation $\left.5 \quad f=\mathrm{a} * \mathrm{e}^{(-\mathrm{b} * \mathrm{x})}\right)$}

\section{Equation $\left.6 \quad f=y 0+\mathrm{a} * \mathrm{e}^{(-\mathrm{b} * \mathrm{x})}\right)$}

with $f$ being the relative chaperone activity or relative refolding at $222 \mathrm{~nm}$ and $x$ reflecting the time of incubation. $y 0$ is the basal relative refolding of inactivated Get3ox. $b$ is the rate constants for the inactivation/refolding kinetic. The halftimes $(\mathrm{t} / 2)$ for the inactivation and refolding were calculated 
from the rate constant $b$ according to equation 4 .

\subsubsection{Chaperone-mediated reactivation of thermally unfolded luciferase}

To test the effect of Get3red and Get3ox on the reactivation of luciferase, $0.1 \mu \mathrm{M}$ of luciferase was incubated either alone or in the presence of a 1:1 molar ratio of either Get3red or Get3ox in $40 \mathrm{mM} \mathrm{mM} \mathrm{MOPS,} 50 \mathrm{mM} \mathrm{KCl}$, $\mathrm{pH} 7.5$ for $15 \mathrm{~min}$ at $43^{\circ} \mathrm{C} .2 \mathrm{mM} \mathrm{DTT}$ were present in the luciferase alone and luciferase plus Get3red samples. $0.1 \mu \mathrm{M}$ of luciferase inactivated in the presence of either $2 \mu \mathrm{M}$ DnaK, $0.4 \mu \mathrm{M}$ DnaJ and $2 \mu \mathrm{M}$ GrpE or $2 \mu \mathrm{M}$ Ydj1 and $1 \mu \mathrm{M}$ HSPA1A served as control. The reaction was cooled down to $25^{\circ} \mathrm{C}$ and after $10 \mathrm{~min}$, the samples were supplemented with $2 \mathrm{mM} \mathrm{MgATP}$ and $0.1 \mathrm{mg} / \mathrm{ml} \mathrm{BSA}$. Where indicated up to $5 \mathrm{mM}$ DTT and either $2 \mu \mathrm{M}$ DnaK, $0.4 \mu \mathrm{M}$ DnaJ and $2 \mu \mathrm{M}$ GrpE or $2 \mu \mathrm{M}$ Ydj1 and $1 \mu \mathrm{M}$ HSPA1A were added. At defined time points, aliquots were taken to measure luciferase activity by luminescence (Herbst et al 1997) using a FLUOstar Omega microplate reader (BMG Labteck).

\subsubsection{ATPase activity assay}

Get3's ATPase activity was monitored with an NADH-coupled ATPase assay in a 96-well BMG FLUOstar Omega microplate reader (Kiianitsa et al 2003). The regeneration system consisting of phosphoenolpyruvate (PEP) and pyruvate kinase $(P K)$ metabolizes one molecule of PEP to pyruvate by converting ADP, generated through ATP hydrolysis, back to ATP. Pyruvate is then reduced to lactate by lactate dehydrogenase (LDH) concomitantly with the oxidation of one NADH molecule (Norby 1988). Hence, the decrease of NADH absorbance at $340 \mathrm{~nm}$ that is coupled to the rate of steady state ATP hydrolysis was monitored in real time. The assay premix consists of $1 \mathrm{mM}$ PEP, $0.5 \mathrm{mM} \mathrm{NADH}, 12 \mathrm{U}$ PK and $22 \mathrm{U} \mathrm{LDH}$ in assay buffer (100 mM HEPES, $10 \mathrm{mM} \mathrm{MgCl}$, 20\% Glycerol, pH 7.0) was added to each well. Subsequently, reduced or oxidized Get3 was diluted to a final concentration of $2 \mathrm{mM}(0.08 \mathrm{mg})$ or $4 \mathrm{mM}(0.16 \mathrm{mg})$ into the premix. Premix without Get3 was used as a blank. The reaction was initiated by adding 
2 mM ATP. The relative ATPase activity was shown as stated in the figure legend.

\subsection{Biochemical analysis of Get3}

\subsubsection{Determination of Get3's redox state}

To determine the number of reduced cysteine thiols in the various Get3 forms, Ellman's assay was used (Riddles et al 1983). Free thiols react with 2,4-dinitro-5-thiobenzoate (DTNB, Ellman's reagent) and cause the stoichiometric cleavage of the disulfide bond to 2-nitro-5-thiobenzo ate (NTB), causing the ionization to the yellow NTB ${ }^{2-}$ dianion. Briefly, modified Get3 protein was diluted to a final concentration of $1 \mu \mathrm{M}$ into $40 \mathrm{mM} \mathrm{KH}_{2} \mathrm{PO}_{4}$ buffer $\mathrm{pH} 7.5$, consisting of $0.17 \mathrm{mg} / \mathrm{ml}$ DTNB and $6 \mathrm{M} \mathrm{Gdn}-\mathrm{HCl}$, to denature the protein and to expose all cysteines to the Ellman's reagent. The buffer alone was used as blank. Protein samples and the blank were incubated for $10 \mathrm{~min}$ at RT in the dark before the absorbance of the suspension was measured in a Jasco spectrophotometer V-550. The amount of accessible thiols was calculated using the extinction coefficient of $13,800 \mathrm{M}^{-1} \mathrm{~cm}^{-1}$ for released NTB, that equals free thiol groups, according to equation 7 :

$$
\begin{array}{ll}
\varepsilon=13800 \mathrm{M}^{-1} \mathrm{~cm}^{-1} \\
\text { Cys }=\frac{\text { A412 blank }- \text { A412 sample }}{\varepsilon * \text { M protein } * l} \quad \mathrm{M}=\text { molarity } \\
\mathrm{I}=\text { path length in } \mathrm{cm}^{2}
\end{array}
$$

\subsubsection{Zinc Determination}

The cysteine-coordinated zinc in Get3 was determined using the PAR/PCMB assay (Jakob et al 2000). Briefly, the assay is based on the complex formation of free zinc and other divalent cations like copper with the dye 4-(2-pyridylazo) resorcinol (PAR, Sigma), which upon complex formation with e.g. zinc results in an intense red color $\left(\varepsilon 500 \mathrm{~nm}\right.$ of $\mathrm{PAR}_{2}(\mathrm{Zn})=$ $\left.66,000 \mathrm{M}^{-1} \mathrm{~cm}^{-1}\right)$. To analyze the amount of free zinc and other surface bound divalent cation, the prepared protein was incubated with $100 \mu \mathrm{M}$ PAR 
in $40 \mathrm{mM}$ metal free $\mathrm{KH}_{2} \mathrm{PO}_{4} \mathrm{pH} 7.5$. Addition of $30 \mu \mathrm{M}$ of the mercury derivative para-chloromercuribenzoic acid (PCMB, ICN Biomedicals) led to the formation of mercaptide bonds with thiols, inducing the release of cysteine bound zinc (Jakob et al 2000). Free zinc is immediately complexed by PAR and the changes in absorbance due to the formation of the red $\mathrm{PAR}_{2}(\mathrm{Zn})$ complex were monitored at a wavelength of $500 \mathrm{~nm}$ in a Jasco spectrophotometer $\mathrm{V}-550$. The amount of released zinc was calculated from a calibration curve for defined concentrations of zinc.

\subsubsection{Analysis for metal content by inductively coupled plasma mass spectrometry (ICP)}

For ICP analysis, all samples were purified twice using Micro Spin 30 columns, equilibrated with metal-free $50 \mathrm{mM}$ Tris, $50 \mathrm{mM} \mathrm{NaCl}, \mathrm{pH} 7.5$ buffer. The ICP analysis was performed in the Keck Elemental Geochemistry Laboratory by Ted Huston (University of Michigan).

\subsection{Monitoring structural changes in Get3}

\subsubsection{Circular dichroism (CD) spectroscopy}

To determine changes in the secondary structure of Get3, far-UV CD spectroscopy was used (Greenfield 2006). In brief, the secondary structure can be monitored due to the unequal absorption of left and right circularly polarized light. Structural elements like alpha-helices and beta-sheets have different characteristic CD spectra; hence any changes in these structural elements can be monitored.

For the CD measurement reduced, oxidized or inactivated Get3 was diluted to a final concentration of $5 \mu \mathrm{M}$ in $20 \mathrm{mM} \mathrm{KH}_{2} \mathrm{PO}_{4}, \mathrm{pH} 7.5$. The far-UV CD spectrum was measured using a Jasco-J810 spectropolarimeter in a quartz cell with $1 \mathrm{~mm}$ path lengths at $20^{\circ} \mathrm{C}$. The $\mathrm{CD}$ spectra were cumulative recorded ( $5 x$ for protein, $3 x$ for buffer) between $260-190 \mathrm{~nm}$, buffer corrected and the molar ellipticity was calculated according to equation 8: 


$$
\begin{aligned}
\theta & =\text { mdeg } \\
\text { molar ellipticity }=\frac{\theta x \mathrm{~m}}{c x l \times 10} \quad \mathrm{~m} & =\text { relative molecular mass } \\
\mathrm{c} & =\text { concentration in } \mathrm{mg} / \mathrm{l} \\
\mathrm{I} & =\text { path length in } \mathrm{deg} * \mathrm{~cm}^{2} * \\
& \mathrm{~d} \text { mole }
\end{aligned}
$$

\subsubsection{Bis-ANS spectroscopy}

To test for the presence of hydrophobic surfaces in Get3, the fluorescence probe 3,3'-Dianilino-1,1'-binaphthyl-5,5'-disulfonic acid (bis-ANS, Molecular Probes) was used as previously described (Graf et al 2004). Reduced, oxidized or inactivated Get3 was diluted to a final concentration of $3 \mu \mathrm{M}$ into $20 \mathrm{mM} \mathrm{KH} 2 \mathrm{PO}_{4}$, pH 7.5 buffer containing $10 \mu \mathrm{M}$ bis-ANS at $30^{\circ} \mathrm{C}$. $\lambda$ ex was set to $370 \mathrm{~nm}$ and the emission spectra was cumulatively recorded (5x for protein, $3 x$ for buffer) from 400 to $600 \mathrm{~nm}\left(/ \lambda_{\text {ex }} / \lambda_{\text {em }}\right.$ slits $\left.2.5 / 5 \mathrm{~nm}, \mathrm{Pmt} 700 \mathrm{~V}\right)$ using a Hitachi F4500 fluorescence spectrophotometer. All spectra were buffer corrected.

\subsection{Purification of various Get3 species and Get3- substrate complex by size-exclusion chromatography (SEC)}

\subsubsection{Analytical gel filtration}

To monitor the oligomerization state of Get3 and its substrate binding, analytical gel filtration was performed. Gel filtration is a standard chromatographic method to separate molecules in solution by their size (Wilton et al 2004). Get3red or Get3ox was applied onto a Superdex 200 10/300 GL column (GE Healthcare), equilibrated with $40 \mathrm{mM}$ HEPES, $140 \mathrm{mM} \mathrm{NaCl}, \mathrm{pH} 7.5$, and eluted with the same buffer at a flow-rate of $0.5 \mathrm{ml} / \mathrm{min}$ using an Äkta-FPLC system (GE Healthcare). The column void volume was determined to be $8.15 \mathrm{ml}$ (Blue Dextran 2,000 kDa) and the 
standards (GE Healthcare) eluted at $9.79 \mathrm{ml}$ (ferritin $440 \mathrm{kDa}$ ), $11.4 \mathrm{ml}$ (aldolase $158 \mathrm{kDa}$ ), $13.22 \mathrm{ml}$ (canalbumin $73 \mathrm{kDa}$ ), $14.61 \mathrm{ml}$ (ovalbumin $43 \mathrm{kDa}$ ) and $16.94 \mathrm{ml}$ (ribonuclease A $13.7 \mathrm{kDa}$ ), respectively. Get3red or Get3ox were loaded at concentrations of $\sim 50 \mu \mathrm{M}$ and $\sim 80 \mu \mathrm{M}$ respectively. Individual fractions of the gel filtration run were collected, pooled and tested for chaperone activity as described. To purify the Get3ox tetramer the protein was loaded at concentrations as high as $\sim 500 \mu \mathrm{M}$ and fractions as small as $200 \mu \mathrm{l}$ containing the Get3ox tetramer were collected.

\subsubsection{Formation of Get $3_{\text {tet-substrate-complexes }}$}

To test complex formation between purified Get3tet and substrate, increasing amounts of Get3 (up to $1.2 \mu \mathrm{M}$ ) were incubated with $0.15 \mu \mathrm{M}$ luciferase in complex buffer ( $40 \mathrm{mM}$ HEPES, $50 \mathrm{mM} \mathrm{KCl}, \mathrm{pH} 7.5$ ), while the temperature was gradually increased from $25^{\circ} \mathrm{C}$ up to $43^{\circ} \mathrm{C}$ over the time course of 22 min. Subsequently, the complexes were concentrated with amicon spin concentrator units (Millipore), applied onto a Superdex 200 gel filtration column, equilibrated with complex buffer, and eluted with the same buffer. To purify large quantities of Get3tet-Luciferase complex, the complex was formed at an 8:1 molar ratio of Get3 to luciferase in complex buffer as described above. The complex-containing mixture was concentrated and processed as outlined in Figure 2.2. To obtain a highly resolved separation of the complex-containing mixture, the concentrated complex was applied onto a micro-preparative Superose6 PC 3.2 (Amersham Pharmacia Biotech) column equilibrated with complex buffer and eluted with the same buffer at a flow-rate of $0.05 \mathrm{ml} / \mathrm{min}$.

\subsubsection{SEC-MALS analysis}

To determine the average molecular weight (MW) of Get3 samples we utilized a WTC-050S5 SEC column (Wyatt Technology Corporation) for separation with an Akta micro (GE Healthcare) equipped with a DAWN HELEOS II multi-angle light scattering (MALS) detector and Optilab rEX differential refractive index detector using ASTRA VI software (Wyatt Technology Corporation). The Get3red, Get3tet and Get3tet-Luciferase 


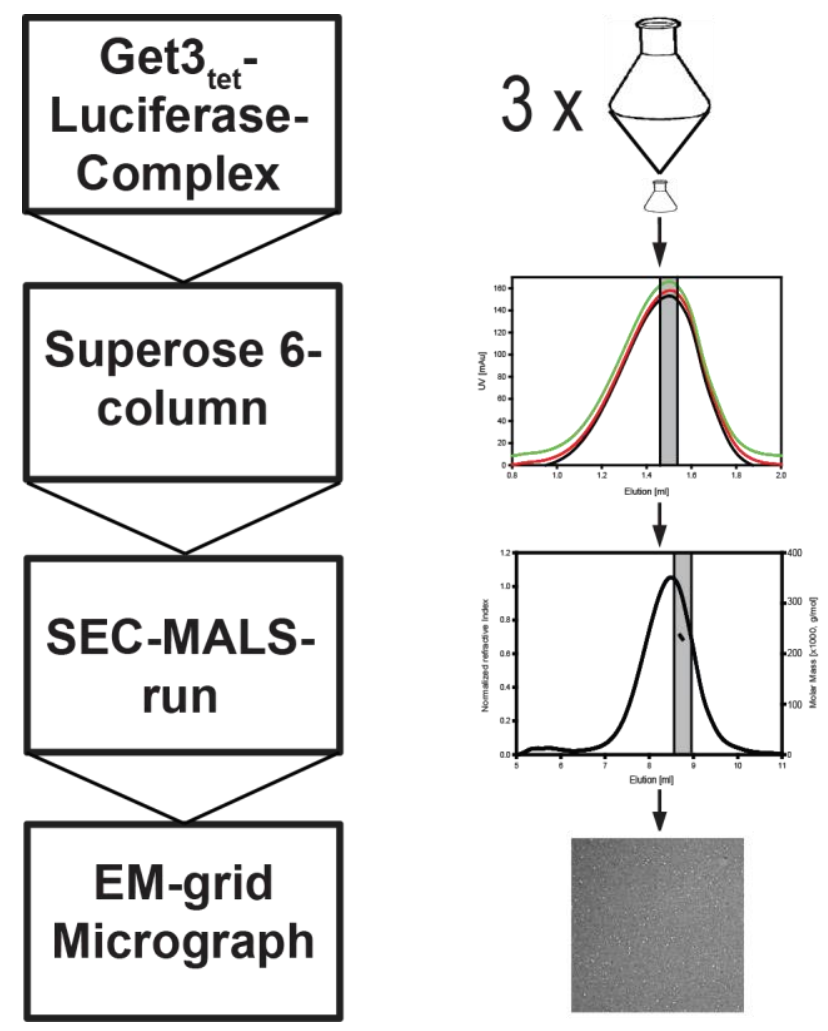

Figure 2. 2: Formation and purification of the $\mathrm{Get}_{\text {tet }}$-Luciferase complex

1) The Get3 $3_{\text {tet }}$-Luciferase complex was formed at an $8: 1$ molar ratio of Get3 $(1.2 \mu \mathrm{M})$ to luciferase $(0.15 \mu \mathrm{M})$ in $16 \mathrm{ml}$ complex buffer (40mM HEPES, $50 \mathrm{mM} \mathrm{KCl}, \mathrm{pH} 7.5)$ three times. Each batch was respectively concentrated down to $0.1 \mathrm{ml}$ and centrifuged for $30 \mathrm{~min}$ at $16,000 \times \mathrm{g}$ to remove aggregates. 2) The three batches of complex were then consecutively applied onto a Superose 6 PC column to separate complexes of various sizes and molar Get3 to luciferase ratios and eluted with complex buffer. The indicated fraction (light gray bar) in the Superose 6 PC elution profile was collected for each run, combined and concentrated downto $0.05 \mathrm{ml}$. 3) Subsequently, the concentrated fraction was run through a size exclusion column connected to a multi-angle light scattering detector (SEC-MALS run) equilibrated with complex buffer, the indicated fraction (light gray bar) was collected and 4) analyzed on a negative stainel ectron microscopy (EM) grid generating 80 micrograph images of the Get3tet-Luciferase complex for single particle analysis.

complex (Get3tet-luc) were run through the SEC-MALS column equilibrated with either $40 \mathrm{mM}$ HEPES, $140 \mathrm{mM} \mathrm{NaCl}$, pH 7.5 for the Get3red/Get3 tet analysis or with $40 \mathrm{mM}$ HEPES, $50 \mathrm{mM} \mathrm{KCl,} \mathrm{pH} 7.5$ for the complex analysis and purification. Indicated fractions were collected and the $\mathrm{Mw}$ was calculated from Raleigh ratio based on the static light scattering and corresponding protein concentration of a selected peak. 


\subsection{Negative-stain EM imaging and analysis}

\subsubsection{Preparation of negative stain EM grids and imaging}

To visualize tetrameric Get3 alone and in complex with substrate, negativestain electron microscopy (EM) was conducted (Ohi et al 2004). Therefore, indicated fractions collected in the SEC-MALS elution were diluted to $\sim 150 \mathrm{nM}$ and stained with $0.75 \%$ uranyl formate $(\mathrm{pH} 5.5-6.0)$ on thin carbonlayered 400 mesh copper grids (Pelco). Samples were then imaged under low dose conditions using a G2 Spirit TEM (FEl) operated at $120 \mathrm{keV}$. Micrographs were taken at 52,000x magnification with 2.16 A per pixel using a $4 \mathrm{k} \times 4 \mathrm{k}$ CCD camera (Gatan).

\subsubsection{Analysis of single particles}

Single particles were selected using E2boxer in EMAN2 (Tang et al 2007) and totaled 39,285 for Get3tet, 7,731 for Get3red and 17,230 for Get3tet-luc. Reference free 2D classification and analysis of Get3 was performed using SPIDER (Frank et al 1996, Radermacher et al 1987) and generated 400 classes for Get3tet, 100 classes for Get3red and 150 classes for Get3tet-luc.

\subsubsection{Refinement of the 3D model}

3D refinement for Get3tet was performed using RELION (Scheres 2012) by first running '3D classification' on the entire data set, binned two-fold, using a sphere as an initial model and no imposed symmetry (Figure 2.3 A). The resulting model was used for an additional 3D classification involving 20 rounds with 3 classes and two-fold symmetry imposed, using the entire unbinned dataset. The best model, based on similarity to the reference-free 2D averages (Figure 3.1. 16), was subjected to additional refinement, using '3D Refine' with two-fold symmetry and converged after 9-rounds, calculated to be $19 \AA$ by the 'gold-standard' Fourier shell correlation procedure (Figure 2.3 B) (Scheres 2012). Additional symmetries were tested, however only the two-fold remained consistent with the asymmetric model and the 2D averages (data not shown). 
A

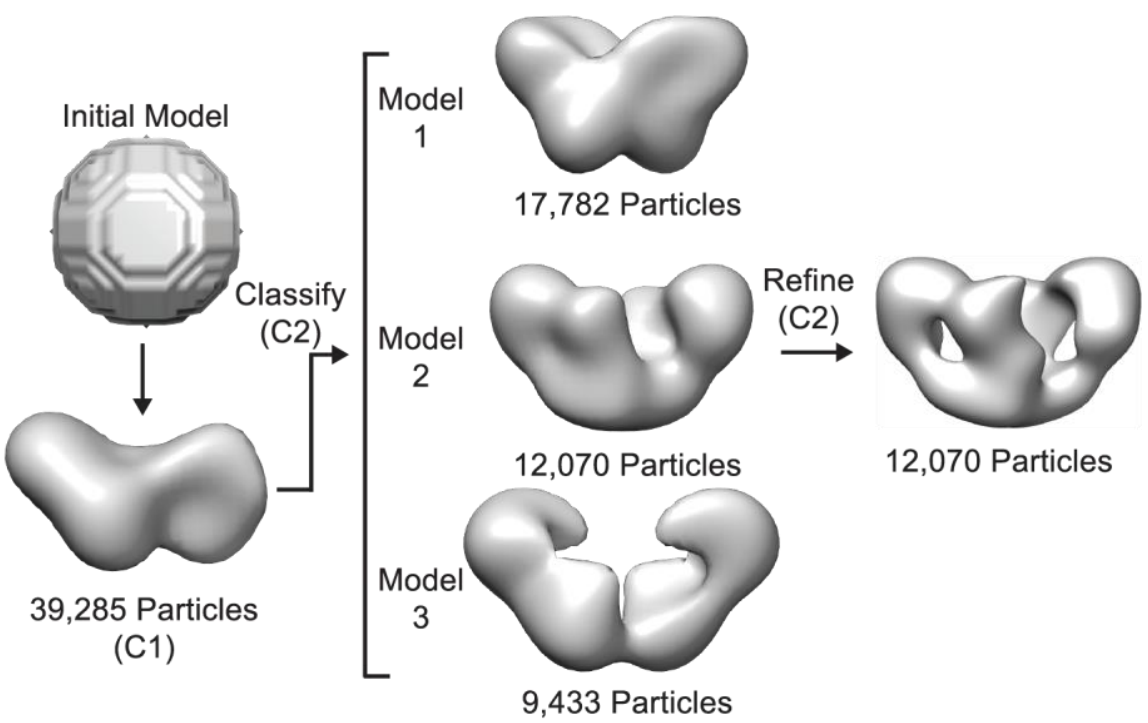

B

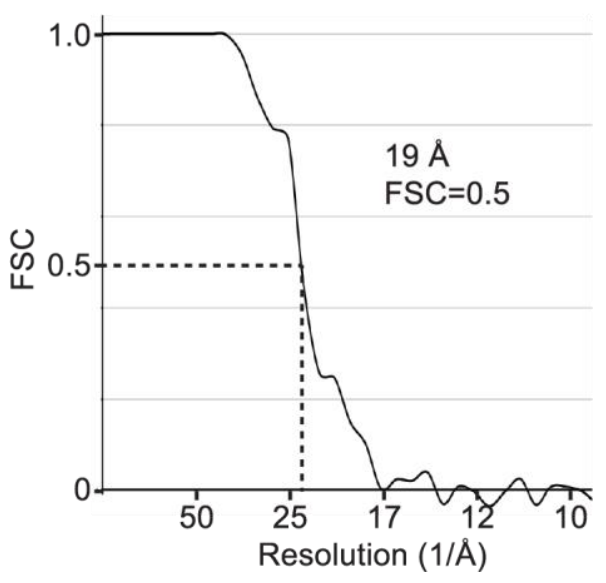

Figure 2. 3: 3D reconstruction of the $\mathrm{Get}_{\text {tet }}$

(A) 3D reconstruction method for the Get3tet. (B) Gold-standard Fourier Shell Correlation (FSC) curve of the final refinement showing a $19 \AA$ resolution at an FSC $=0.5$ criterion, performed using RELION (Scheres 2012).

\subsection{Hydrogen/Deuterium exchange experiments combined with mass spectrometry}

\subsubsection{Peptide fragment optimization and MS analysis}

Pepsin digestion conditions for Get3 were optimized as previously described and the resulting peptide fragments were analyzed by mass 
spectroscopy (MS) (Marsh et al 2013). Briefly, Get3red and Get3ox were digested in the presence of various concentrations of guanidiumhydrochloride containing quench buffer (0.8\% formic acid, $16.6 \% \mathrm{v} / \mathrm{v}$ glycerol) in the presence or absence of $100 \mathrm{mM}$ of the reducing agent tris(2carboxyethyl)phosphine (TCEP). The resulting peptides were subjected to liquid chromatography (LC)-MS analysis and selected MS spectra were shown in Figure 3.1. 7. A greater than 99.8\% peptide coverage map for Get3 was obtained using quench buffer containing $1 \mathrm{M} \mathrm{Gdn-HCl}$ and $100 \mathrm{mM}$ TCEP.

\subsubsection{Deuterium-hydrogen exchange}

The H/D exchange experiments were initiated by diluting $45 \mu \mathrm{l}$ of reduced Get3red $(\sim 36 \mu \mathrm{M})$ or Get3ox $(\sim 32 \mu \mathrm{M})$ with $135 \mu \mathrm{l}$ of $\mathrm{D}_{2} \mathrm{O}$ buffer (8.3 mM Tris, $50 \mathrm{mM} \mathrm{NaCl}$, in $\mathrm{D}_{2} \mathrm{O}$, pDread 7.2) at $0^{\circ} \mathrm{C}$. At 10, 30, 100, 300, 1000, 3000, and $10000 \mathrm{sec}, 24 \mu \mathrm{l}$ of the reaction were removed and quenched by adding $36 \mu \mathrm{l}$ of optimized quench buffer (1.0 M Gdn- $\mathrm{HCl}, 100 \mathrm{mM}$ TECP, $0.8 \%$ formic acid, $16.6 \% \mathrm{v} / \mathrm{v}$ glycerol) at $0^{\circ} \mathrm{C}$. The quenched samples were incubated on ice for $5 \mathrm{~min}$, and then frozen at $-80^{\circ} \mathrm{C}$. In addition, nondeuterated control samples (incubated in non-deuterated $\mathrm{D}_{2} \mathrm{O}$ buffer) and equilibrium-deuterated back exchange control samples (incubated in $\mathrm{D}_{2} \mathrm{O}$ buffer containing $0.5 \%$ formic acid overnight at $25^{\circ} \mathrm{C}$ ) were prepared. All samples were subsequently digested and analyzed by mass spectrometry.

\subsubsection{Data analysis}

The deuteration level for each peptide was calculated according to equation 9 :

$$
\text { Deuteration }(\%)=C(P)-C(N)) /(C(F)-C(N)) \times C(N) \times \operatorname{MaxD} \times 100
$$

where $C(P), C(N)$ and $C(F)$ are the centroid values of partially deuterated peptide, non-deuterated peptide, and fully deuterated peptide at each time point. MaxD is the maximum deuterium incorporation for each peptide. It is calculated as the number of amino acids in the peptide minus 2 minus any proline that is present beyond position 2 of the peptide. 


\subsection{Microbiological methods}

\subsubsection{Yeast transformation}

Yeast strains and plasmids used in this study are listed in chapter 2.1. For yeast transformation a modified protocol for the lithium method was used (Ito et al 1983). Briefly, $3 \mathrm{ml}$ of an overnight culture of a yeast strain grown in YPAD media (10 g/l Bacto yeast extract, $20 \mathrm{~g} / \mathrm{l}$ Bacto peptone, $20 \mathrm{~g} / \mathrm{l}$ Glucose, $40 \mathrm{mg} / \mathrm{l}$ adenine sulfate) was pelleted and washed with doubledistilled $\mathrm{H}_{2} \mathrm{O}$. The cell pellet was resuspended in $1.4 \mathrm{ml}$ transformation buffer (10 mM Tris pH 7.5, 1 mM EDTA, $100 \mathrm{mM} \mathrm{LiAc,} \mathrm{35 \%} \mathrm{PEG} \mathrm{(v/v))}$ containing $18 \mu \mathrm{l}$ carrier DNA and $0.5 \mu \mathrm{g}$ of plasmid DNA. The mixture was vortexed extensively, incubated at RT for $1 \mathrm{~h}$ and heat-shocked at $42^{\circ} \mathrm{C}$ for 15 min. The transformation buffer was removed by centrifugation and the cells were spread on synthetic complete (SC) agar plates (6.7 g/l Bactoyeast nitrogen base w/o amino acids, $20 \mathrm{~g} / \mathrm{l}$ Glucose, $2 \mathrm{~g} / \mathrm{l}$ dropout mix, $20 \mathrm{~g} / \mathrm{l} \mathrm{Bacto}$ Agar) missing the plasmid specific amino acid for selection of transformed cells.

\subsubsection{Growth conditions for yeast}

Cells were grown in Hartwell's Complete (HC) medium (Burke et al 2000) and experiments were performed at mid-log phase (optical density (OD)600 of 0.4 - 0.8). To determine the sensitivity of yeast strains, overnight cultures were diluted in $\mathrm{HC}$ medium to an $\mathrm{OD} 600$ of 0.4 . Subsequently serial dilutions (1:5 dilutions in each step) of each culture were spotted on $\mathrm{HC}$ agar plates in the absence or presence of either $1.5 \mathrm{mM} \mathrm{H}_{2} \mathrm{O}_{2}$ or $1 \mathrm{mM} \mathrm{CuSO}_{4}$ and grown for 2-3 days at the indicated temperatures.

\subsubsection{Live Cell Fluorescence microscopy}

Images of yeast cells were acquired on a Delta Vision RT (Applied Precision) microscope using a $100 \times / 0.35-1.5$ Uplan Apo objective and specific band pass filter sets for GFP or mCherry. The images were acquired using a Coolsnap $\mathrm{HQ}$ (Photometrics) camera. Image processing was 
performed using ImageJ (http://rsbweb.nih.gov/ij/). Pixel fluorescence intensity of at least 40 cells per sample was quantified as described in (Jonikas et al 2009, Vilardi et al 2014) using Knime software (www.knime.org/knime).

\subsubsection{Analysis of Get3 expression levels}

$1 \mathrm{ml}$ of mid-log phase yeast culture was pelleted by low speed centrifugation (5 min at $1,000 \times \mathrm{g}$ ), resuspended in fresh $0.1 \mathrm{M} \mathrm{NaOH}$ and incubated for ten minutes at room temperature. Cells were recovered by centrifugation, dissolved in $1 \mathrm{x}$ reducing Laemmli buffer and boiled for $5 \mathrm{~min}$ at $95^{\circ} \mathrm{C} .10 \mu \mathrm{l}$ of sample per lane were separated by SDS-PAGE and analyzed by immunoblot as previously described.

Alternatively, for Get3 expression levels shown in Figure 3.1.1, $20 \mathrm{ml}$ of midlog phase yeast culture (synchronized OD when comparing expression levels of different strains) was pelleted (5 min at 1,000 $\mathrm{xg}$ ) and resuspended in $200 \mu \mathrm{l}$ lysis buffer $(20 \mathrm{mM}$ Hepes pH 7.5, $100 \mathrm{mM} \mathrm{NaCl}$ ) supplemented with $1 \mathrm{mM}$ PMSF in $1.5 \mathrm{ml}$ Eppendorf tubes. At least $200 \mu \mathrm{l}$ of glass beads ( $0.5 \mathrm{~mm}$ diameter, Biospec) were added to the cell-suspension. The tubes were then vortexed for $5 \mathrm{~min}$ at (1400 rpm) with $5 \mathrm{~min}$ breaks in between for a total of $15 \mathrm{~min}$ at $4^{\circ} \mathrm{C}$. The beads and intact cells were removed by low speed centrifugation ( $1 \mathrm{~min}$ at $1,000 \times \mathrm{g}$ ). The "cleared" lysate was diluted 1:10 in $1 \mathrm{x}$ reducing Laemmli buffer and boiled for $5 \mathrm{~min}$ at $95^{\circ} \mathrm{C} .10 \mu \mathrm{l}$ of sample per lane were separated by SDS-PAGE and analyzed by immunoblot as previously described. 


\section{Results}

\subsection{The protein targeting factor Get3 functions as ATP- independent chaperone under oxidative stress conditions}

Previous studies conducted in yeast suggested a second function for the TA-protein targeting factor Get3 as an ATP-independent chaperone holdase. Get3 has a copper-sensitive phenotype in vivo, is able to chaperone soluble proteins in vitro and, under conditions of energy depletion, colocalizes with other chaperones to sites of protein aggregates (Metz et al 2006b, Powis et al 2013b). Furthermore, there is a striking similarity between Get3's highly conserved cysteine motif and the redox sensitive cysteine motif found in the redox-regulated chaperone heat-shock protein 33 (Hsp33), which is capable of forming disulfide bonds (Graf et al 2004). Surprisingly, in 2009 Suloway et al. crystallized disulfide-linked Get3 dimers. Taken together, these findings raised the possibility that Get3 similar to Hsp33 might serve as a redox-controlled chaperone that protects proteins against oxidative stress-induced protein aggregation. Under these stress conditions, ATP-independent chaperones are particularly crucial since reactive oxygen species (ROS) cause a severe and rapid decrease in

Most of the results in the chapter 3.1 were published in: Voth W, Schick M, Gates S, Li S, Vilardi F, Gostimskaya I, Southworth DR, Schwappach B, Jakob U. The protein targeting factor Get3 functions as ATP-independent chaperone under oxidative stress conditions. Mol Cell 2014;56:116-27. Some results were obtained under my supervision by an undergraduate student, M. Schick, or in close collaboration with scientists participating in the study: Copper chelating experiments in Figure 3.1.4, initial inactivation experiments for Figure 3.1.10, CD experiments in Figure 3.1.11 and bis-ANS measurements in Figure 3.1.12 A were performed by Schick M under the supervision of Voth W. Furthermore, most of the micrographs taken and particles picked in Figure 3.1.15, all of the class averages, refinement processes and 3D modeling in Figure 3.1.16 by Gates $S$ (Southworth Laboratory, University of Michigan). Mass spectrometry runs in Figure 3.1.7 and Figure 3.1.17, HD/EX experiments in Figure 3.1.18 - 19 were performed by Li S (University of California San Diego School of Medicine) and analyzed by Voth W. In vivo experiments and microscopy in Figure 3.1.22 - 24 were performed by Vilardi $F$ (Schwappach Laboratory, Universitätsmedizin Göttingen). The manuscript was written by Voth W, Southworth DR, Schwappach B and Jakob U. 
cellular ATP-levels, thereby incapacitating ATP-dependent chaperones (Shenton \& Grant 2003, Winter et al 2005).

\subsubsection{Get3 - A redox-regulated chaperone in eukaryotes}

To test whether sensitivity of get3 cells towards various oxidative stresses in vivo (Schuldiner et al 2008, Shen et al 2003b) is connected to Get3's cysteines, I conducted survival assays with different Get3 cysteine mutants. Under normal growth conditions the get3 deletion mutants that were transformed with either an empty construct or various Get3 mutant variants lacking up to five cysteines showed no visible growth defect (Figure 3.1.1 A). However, in agreement with previous findings, exposure of get3 cells to oxidative stress conditions, such as induced by heat shock or copper and hydrogen peroxide stress led to severe growth defects that were completely reversed when wild-type Get3 was expressed from a plasmid. The mutant variant of Get3 C36T, C85T, C317T ( $\triangle \mathrm{CCC}$ ) lacking the three non-conserved cysteines, rescued the get3 phenotype to the same extent as wild-type Get3 (Figure 3.1.1 A). In contrast, expression of either Get3 C36T, C85T, C240T, C242T, C317T ( $\triangle \mathrm{CVC}$ ) or Get3 C36T, C85T, C285T, C288T, C317T ( $\triangle \mathrm{CXYC)}$ mutant variants, which each lack one of the conserved cysteine pairs and the non-conserved cysteines, exhibited growth defects under both copper stress and heat shock conditions (Figure 3.1.1 A). Notably, mutations of the zinc-coordinating cysteines (Cys 285/288) led to decreased expression levels of Get3 $\triangle$ CXYC in the cell (Figure 3.1.1 B). This result might explain the very severe growth defect under stress conditions. These findings suggest that at least some of Get3's conserved cysteines are essential for the oxidative stress survival of yeast cells, and might form the basis of a redox-regulated switch altering Get3's function.

To test if Get3's previously observed general chaperone activity was indeed redox-regulated, I purified wild-type Get3 under reducing conditions. Additional incubation of purified Get3 with reducing agent (5 mM DTT) ensured a fully reduced protein (Get3red). Subsequently, I tested Get3 for its ability to prevent the aggregation of chemically or thermally unfolding client 
A

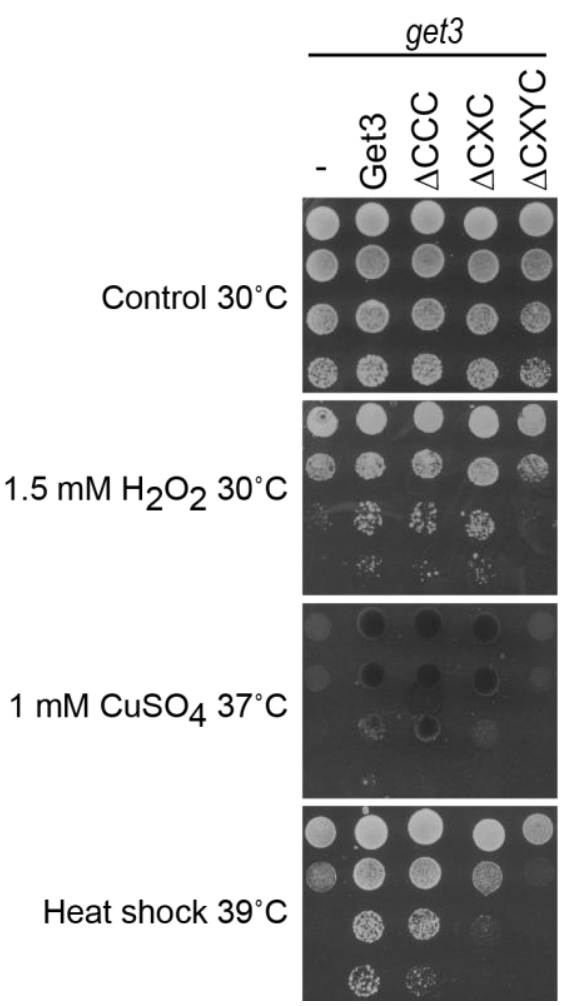

B

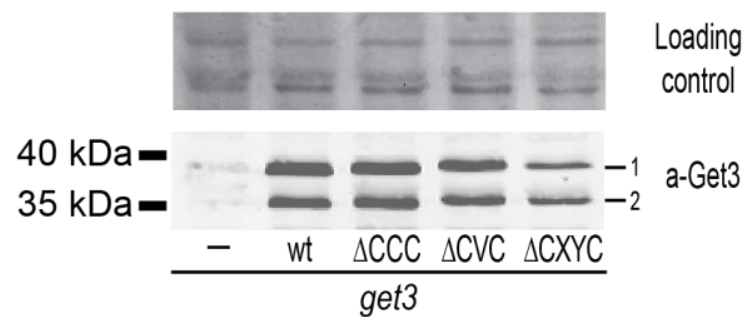

$\triangle C C C=$ Get3 C36T, C85T,C317T

$\triangle C V C=$ Get3 C36T,C85T,C240T,C242T,C317T

$\triangle C X Y C=$ Get3 C36T,C85T,C285T,C288T,C317T

Figure 3.1. 1: Get3's conserved cysteines are essential for yeast cell survival under oxidative stress conditions

(A) get3 cells were transformed with an empty construct or with constructs containing the coding sequence of wild-type or mutant variants of Get3. Serial dilutions were then spotted on control plates containing $1.5 \mathrm{mM} \mathrm{H}_{2} \mathrm{O}_{2}$ or $1 \mathrm{mM}$ of $\mathrm{CuSO}_{4}$. Subsequently the plates were incubated at the indicated temperatures. (B) get3 cells were transformed with constructs containing the coding sequence of wild-type or mutant Get3 variants. Cells were lysed by bead beating and the lysates were separated by reducing SDS-PAGE and analyzed by immunoblot using a Get3 specific serum. Lysis by beat beating leads to a typical partial degradation product (2) of full-length Get3 (1), most likely due to the activation of vacuolar proteases, resulting in a characteristic double band when analyzed by immunoblotting. The ponceau $S$ stained blot was used as loading control. Full descriptions for the abbreviated cysteine mutants is shown.

proteins, such as citrate synthase (CS) and luciferase, commonly used in vitro chaperone client proteins. For the chemical aggregation assays, client proteins (CS or luciferase) are denatured in $6 \mathrm{M}$ guanidine hydrochloride (Gdn-HCl) overnight, and diluted into buffer leading to the rapid formation of aggregates that can be monitored by light scattering (Figure 3.1.2 A and B, control). In contrast, thermal unfolding and aggregation of the thermally instable luciferase or citrate synthase is a rather slow process and induced 
A

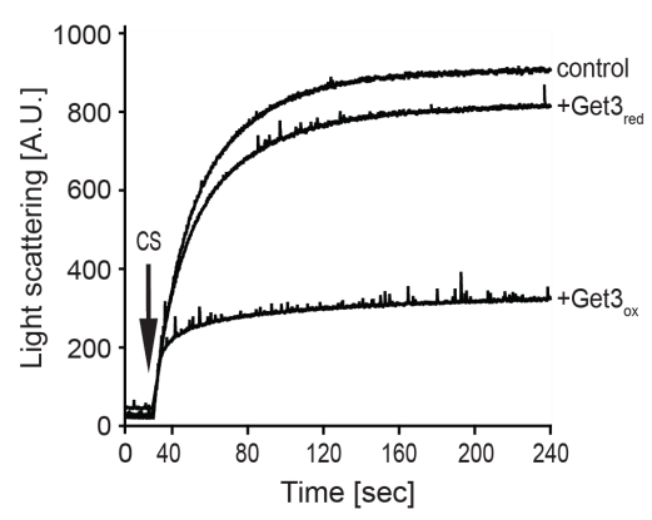

C

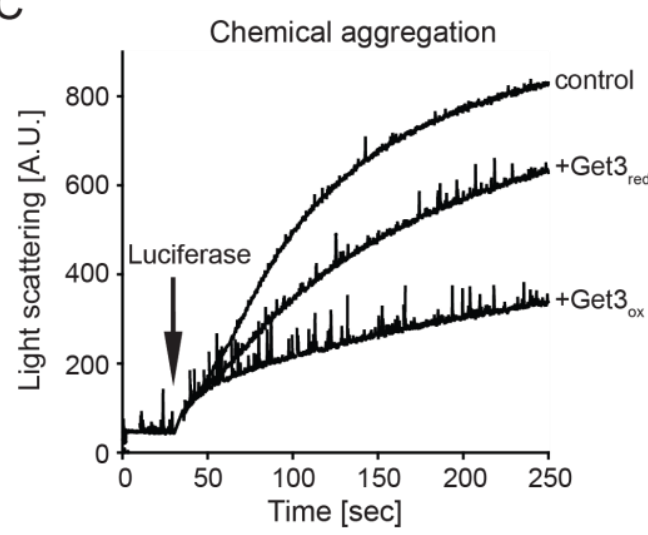

B
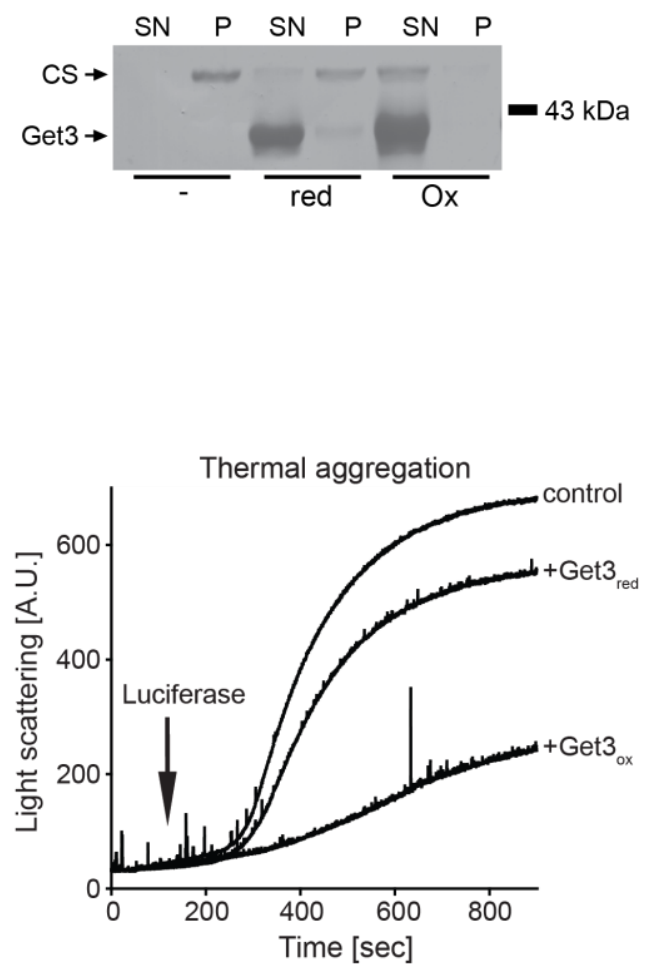

Figure 3.1. 2: Get3 exhibits chaperone activity when oxidized

(A) Effect of an 8-fold molar excess of Get $3_{\text {red }}$ or Get $3_{\text {ox }}$ on the light scattering of $75 \mathrm{nM}$ chemically denatured citrate synthase (CS) upon dilution into buffer. Light scattering signal of aggregating CS measured in the absence of added chaperones is shown as control. (B) After $240 \mathrm{sec}$ samples were pelleted by centrifugation. Subsequently the soluble fraction in the supernatant $(\mathrm{SN})$ and the insoluble fraction in the pellet $(\mathrm{P})$ were analyzed on reducing SDS-PAGE. (C) Influence of Get3 red $_{\text {or }}$ Get $3_{\text {ox }}$ on the light scattering of chemically denatured luciferase (Get3:luciferase ratio 8:1) or thermally unfolding luciferase at $43^{\circ} \mathrm{C}$ (Get3:luciferase ratio 1:1) upon dilution into $40 \mathrm{mM} \mathrm{MOPS}, 50 \mathrm{mM} \mathrm{KCl}$. $\mathrm{pH} 7.5$ buffer. Light scattering of aggregating luciferase measured in the absence of added chaperones is shown (control).

by incubation of the enzymes at heat shock temperatures (i.e., $43^{\circ} \mathrm{C}$ ). As shown in Figure 3.1.2, an eight-fold molar excess of Get3red over CS had only a minor effect on the aggregation behavior of chemically unfolding CS. In contrast, Get3red incubated with the oxidant hydrogen peroxide $\left(\mathrm{H}_{2} \mathrm{O}_{2}\right)$ and $\mathrm{Cu}^{2+}$ resulted in a highly chaperone active protein (Get3ox) that almost completely inhibited the CS aggregation at an 8:1 molar ratio of Get3ox to CS (Figure 3.1.2 A). Samples tested in the light scattering assay were 


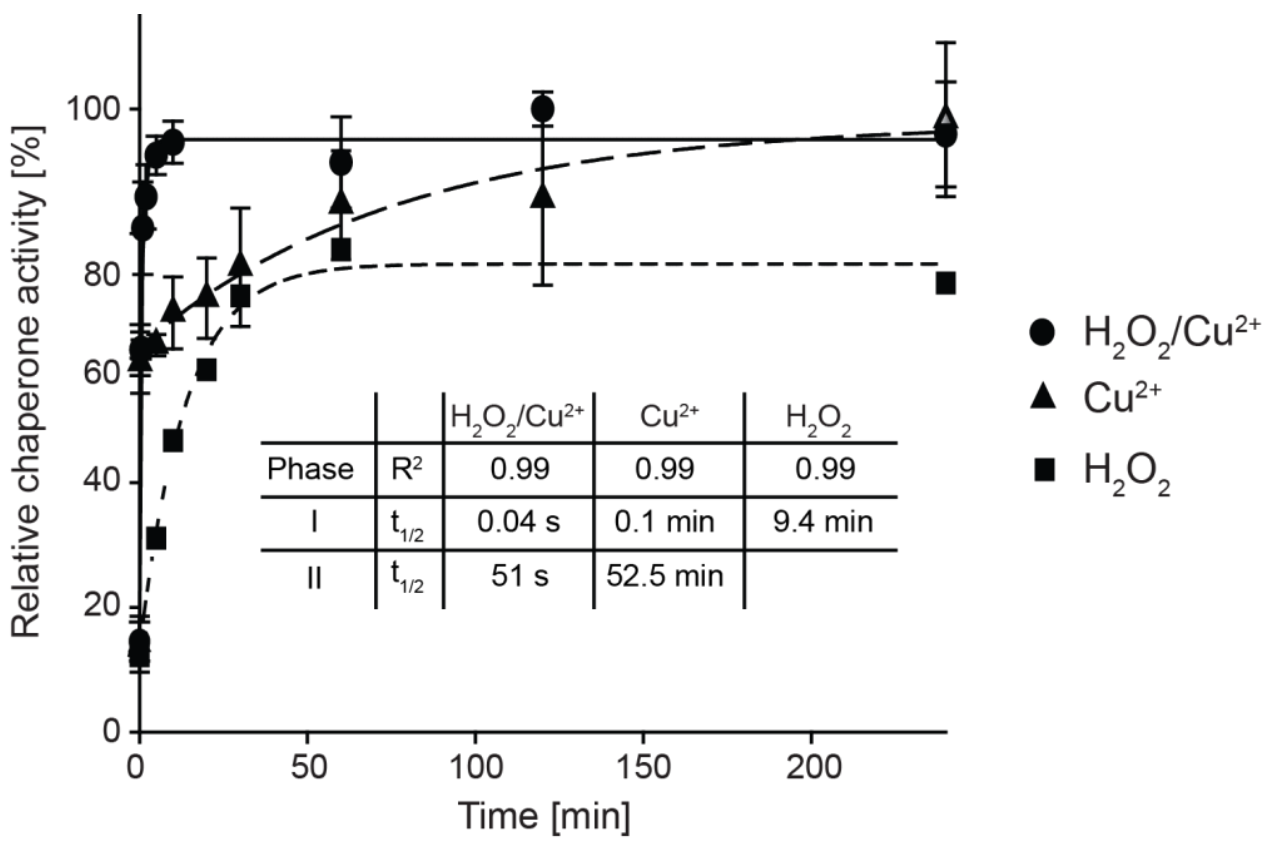

Figure 3.1. 3: ROS-mediated activation of Get3's chaperone function

The chaperone activity of $0.3 \mu \mathrm{M} \mathrm{Get} 3_{\text {red }}$ before and at defined time points after incubation in either $2 \mathrm{mMH}_{2} \mathrm{O}_{2} / 50 \mu \mathrm{M} \mathrm{Cu}^{2+}$ (circles), $50 \mu \mathrm{m} \mathrm{Cu}^{2+}$ (triangles) or $2 \mathrm{mM} \mathrm{H}_{2} \mathrm{O}_{2}$ (squares) at $37^{\circ} \mathrm{C}$ was determined by analyzing the influence of Get3 on the aggregation of $0.075 \mu \mathrm{M}$ chemically denatured CS. The light scattering signal of aggregated CS in the absence of added chaperones was set to $0 \%$ chaperone activity, while the light s cattering signal in the presence of fully oxidized Get 3 was set to $100 \%$. For details, see the material and methods part. Insert: The data points were fitted to an exponential function using double exponential fitting for $\mathrm{H}_{2} \mathrm{O}_{2} / \mathrm{Cu}^{2+}$ and $\mathrm{Cu}^{2+}$ or single exponential fitting for $\mathrm{H}_{2} \mathrm{O}_{2}$ in SigmaPlot V13. The halftimes for the activation of Get3 were obtained from the fit (see methods part for details). At least 3-6 replicates for $\mathrm{H}_{2} \mathrm{O}_{2} / \mathrm{Cu}^{2+}$ and $\mathrm{Cu}^{2+}$ activation were performed and the standard error(SE) is shown.

subsequently analyzed for their solubility using spin down assays. CS diluted into buffer containing oxidized Get3 remained completely soluble, whereas CS diluted into buffer containing reduced Get3 was almost exclusively found in the insoluble fraction very similar to CS diluted into buffer without any chaperones (Figure 3.1.2 B). I obtained comparable results when I tested the influence of Get3red and Get3ox on chemically unfolding luciferase or thermally unfolding luciferase (Figure 3.1.2 C). In all cases, Get3ox showed a significantly higher chaperone activity compared to Get3red. These results suggest that incubation of Get3 with hydroxylproducing oxidants converts reduced chaperone-inactive Get3 into a general molecular chaperone for unfolding soluble proteins. 
Consistent with previous studies on the redox regulated chaperone Hsp33 (Graumann et al 2001, Pecci et al 1997), activation of Get3's chaperone function with the hydroxyl-radical producing mixture of $\mathrm{H}_{2} \mathrm{O}_{2}$ and $\mathrm{Cu}^{2+}$ was very rapid and went to completion within minutes of the incubation at $37^{\circ} \mathrm{C}$ ( $\mathrm{T}_{1 / 2}<1 \mathrm{~min}$ ) (Figure 3.1.3, black circles). In contrast, incubation of Get3red with $\mathrm{H}_{2} \mathrm{O}_{2}$ in the absence of copper yielded a much lower chaperone activation, and slower activation kinetics ( $\mathrm{T}_{1 / 2} \sim 9.5 \mathrm{~min}$ ) (Figure 3.1.3, black squares). Surprisingly, incubation of Get3red with $50 \mu \mathrm{M} \mathrm{Cu}{ }^{2+}$ in the absence of $\mathrm{H}_{2} \mathrm{O}_{2}$ also activated the chaperone function of Get3. The displayed kinetics suggested an extremely fast initial activation (Phase I, $\mathrm{T}_{1 / 2}<6 \mathrm{sec}$ ), followed by a slower activation phase (Phase II, $\mathrm{T}_{1 / 2}<\sim 50 \mathrm{~min}$ ) (Figure 3.1.3, black triangles). Since get3 mutants show sensitivity towards copper in vivo we explored the possibility that copper might be a direct activator of Get3's chaperone function. We thus tested whether increasing concentrations of $\mathrm{Cu}^{2+}$ can activate Get3's chaperone activity more efficiently. Indeed, at the highest $\mathrm{Cu}^{2+}$ concentrations tested $(200 \mu \mathrm{M}$ and $400 \mu \mathrm{M})$, full activation of Get3's chaperone activity was achieved within the mixing time of the experiment (Figure 3.1.4 A). To test whether it is $\mathrm{Cu}^{2+}$ binding that leads to the activation of Get3, we utilized the strong $\mathrm{Cu}^{2+-}$-chelator tetrakis(2pyridylmethyl) ethylene-diamine (TPEN) to remove all copper. We therefor incubated Get3 probes activated with $200 \mu \mathrm{M} \mathrm{Cu}^{2+}$ (green bars) and $400 \mu \mathrm{M} \mathrm{Cu}^{2+}$ (red bars) with TPEN immediately before testing Get3's chaperone activity (Figure 3.1.4 B). In parallel, we performed metal analysis of the probes using inductively coupled plasma mass spectrometry (ICP), which confirmed that TCEP effectively removed nearly all the $\mathrm{Cu}^{2+}$ (Figure 3.1.4 B, remaining $\mathrm{Cu}^{2+}$ ). The chaperone assay revealed no difference in the chaperone activity of Get3 upon removal of copper (Figure 3.1.4 B). These experiments indicate that the activation of Get3's chaperone function is not triggered by copper binding but is likely due to oxidative modification of Get3. In agreement with this result, we found that Get3 activated with $\mathrm{Cu}^{2+}$ under anaerobic conditions exhibited no difference in oxidation state and chaperone activity compared to Get3 activated with $\mathrm{Cu}^{2+}$ under aerobic conditions (Figure 3.1.6, compare light gray and dark gray bars). 
A

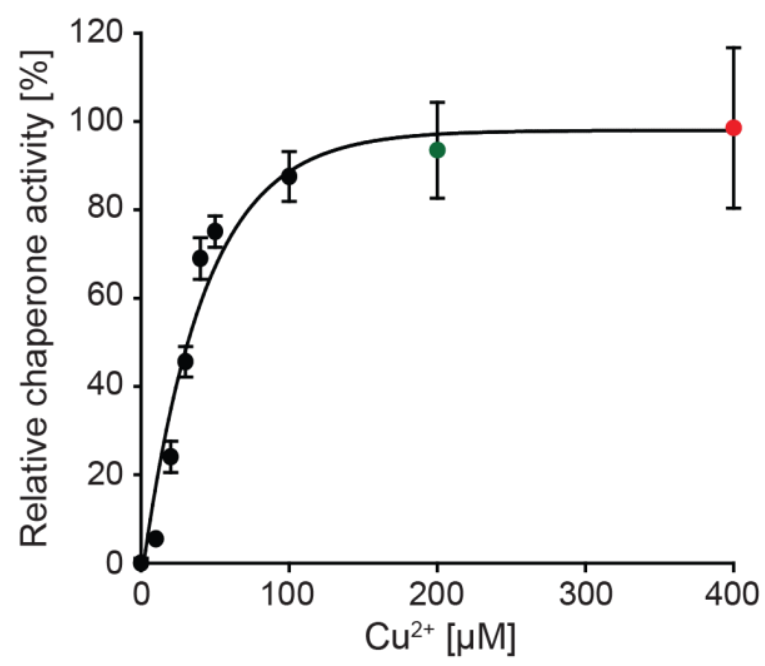

B

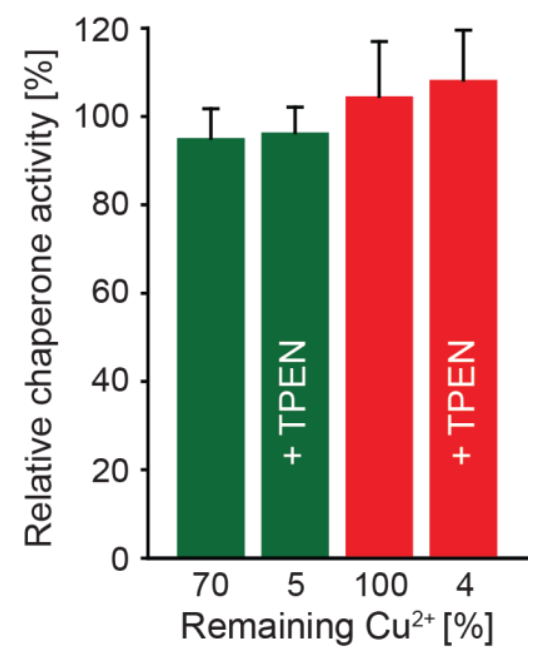

Figure 3.1. 4: Copper activates Get3's chaperone function through oxidation

(A) The chaperone function of $0.3 \mu \mathrm{M}$ Get $3_{\text {red }}$ before and 4 min after addition of increasing concentrations of copper at $37^{\circ} \mathrm{C}$ was analyzed in the chaperone assay with chemically denatured CS as previously described. (B) Get $3_{\text {red }}$ activated for 4 min in the presence of $200 \mu \mathrm{M}$ (green bars) or $400 \mu \mathrm{M} \mathrm{CuCl}_{2}$ (red bars) was either incubated with a 100 -fold excess of the strong copper chelator TPEN for $10 \mathrm{~min}$ or left untreated. The samples were then loaded onto gel filtration columns equilibrated with metal-free buffer and subsequently tested for their remaining copper content by inductively coupled plasma mass spectrometry (ICP) analysis. The same samples were tested for their chaperone activity in the CS chaperone assay as described above. At least 3 replicates were performed and the SE is shown.

In previous studies, Get3's ability to hydrolyze ATP was shown to be directly connected to its TA-protein shuttling function (Rome et al 2013). Therefore, we investigated whether Get3's ATPase function is also connected to its chaperone activity. To test whether oxidation affects the ATPase activity of Get3, I compared the ATPase activity of oxidized and reduced Get3. I observed that the ATPase activity of chaperone-active Get3ox is decreased to less than $20 \%$ compared to Get3red (Figure 3.1.5 B, light gray bars), suggesting that oxidation of Get3 leads to conformational changes that affect ATP hydrolysis. These findings are fully consistent with previous results, which showed that Get3's chaperone function is not affected by the presence of ATP and that under ATP-depleting conditions in vivo, Get3 colocalizes with protein aggregates (Powis et al 2013b). To further test whether Get3's ATPase function influences its oxidative activation as a 
chaperone, I analyzed the chaperone function of the ATPase-deficient Get3 D57E mutant variant, which was shown to have a five-fold lower affinity for $\mathrm{Mg}^{2+}$ and a drastically reduced ATPase activity in ArsA, the prokaryotic homologue of Get3 (Figure 1.6) (Zhou et al 2000). The mutant variant, which had negligible ATPase activity in the reduced or oxidized state, was indeed fully chaperone-active when oxidized and showed chaperone activity similar to oxidized wild-type Get3 (Figure 3.1.5, compare light and dark gray bars). These results suggest that ATP-hydrolysis is neither required for the oxidative activation of Get3 nor for its general chaperone function. In conclusion, these data indicate that Get3 rapidly converts into a potent ATPindependent chaperone when exposed to oxidative stress conditions in vitro.
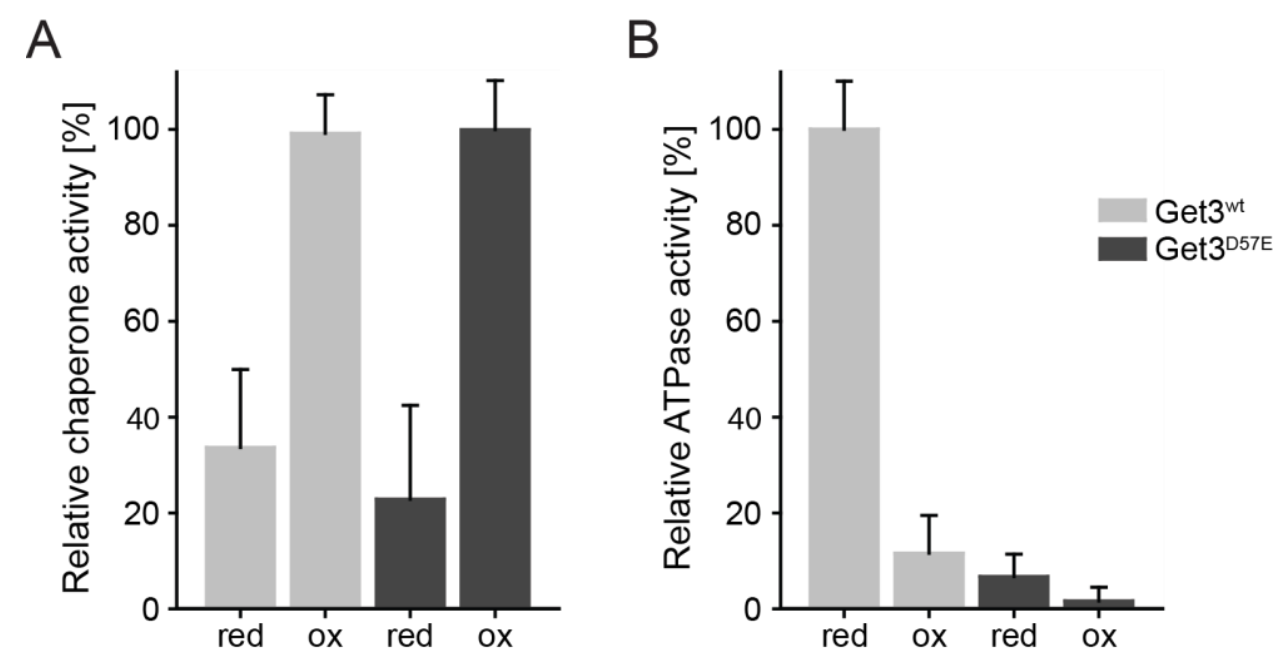

Figure 3.1. 5: Chaperone activity of oxidized Get 3 is ATP-independent

(A) The relative ATPase activity of reduced and oxidized wild-type Get3 (light gray bars) and the ATPase deficient Get3 D57E mutant (dark gray bars) is shown. (B) The same samples were tested for their relative chaperone activity in the CS chaperone assay as described above. A four-fold excess of Get 3 to CS was used for the chaperone assays. At least 3-6 replicates were performed and the SE is shown.

\subsubsection{Mechanism of Get3's activation process}

Mixtures of hydrogen peroxide and copper produce highly reactive hydroxyl radicals that rapidly oxidize cysteine thiols. $\mathrm{Cu}^{2+}$, on the other hand, is known to directly catalyze the auto-oxidation of cysteine thiols with biphasic 
A

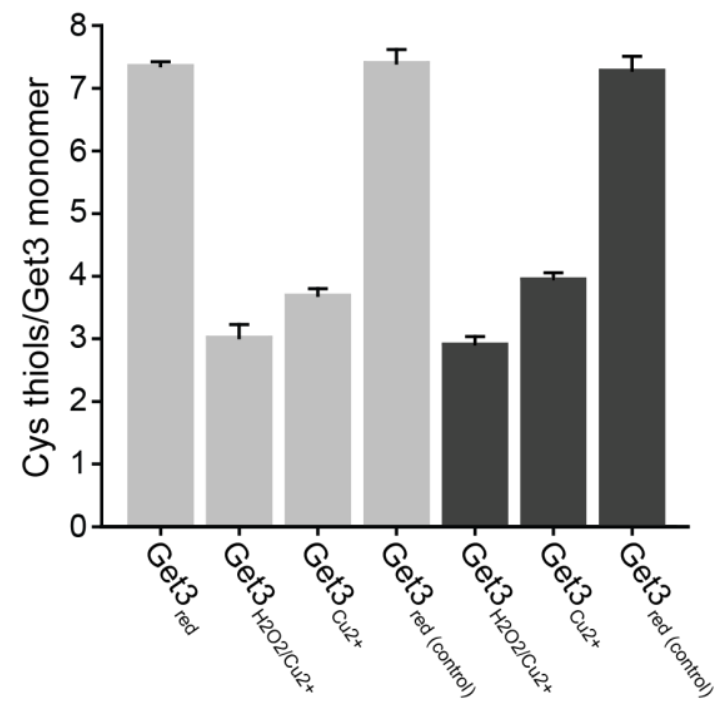

B

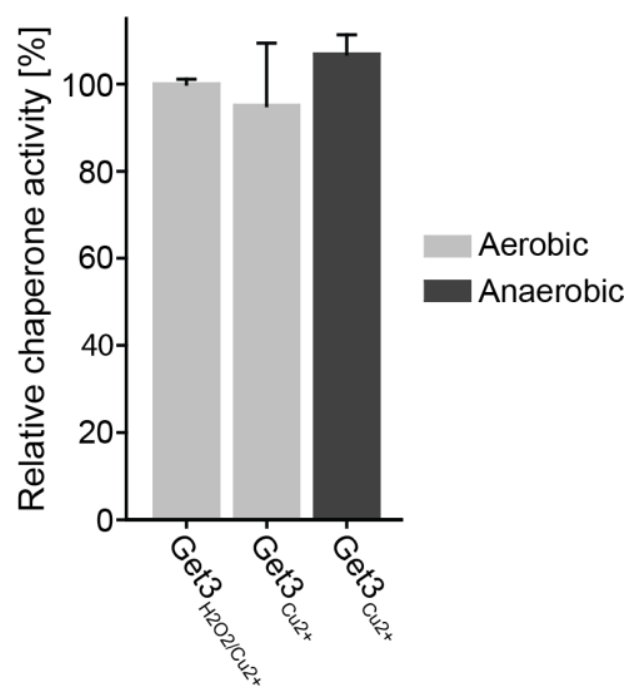

Figure 3.1. 6: Get3's chaperone function is activated through thiol oxidation

(A) Get3 $3_{\text {red }}$ was incubated in the presence of either $2 \mathrm{mM} \mathrm{H}_{2} \mathrm{O}_{2} / 50 \mu \mathrm{MCu}^{2+}$ or $50 \mu \mathrm{MCu}^{2+}$ at $37^{\circ} \mathrm{C}$ for $4 \mathrm{~h}$ under aerobic (light gray bars) and anaerobic (dark gray bars) conditions. The oxidants were removed by gel filtration and subsequently the number of cysteine thiols in reduced and oxidized Get3 was determined under denaturing conditions u sing the Ellman's assay. Get3 $3_{\text {red }}$ alone incubated in the absence of oxidants at $37^{\circ} \mathrm{C}$ for $4 \mathrm{~h}$ served as control to exclude air oxidation. (B) Subsequently, the samples treated with $50 \mu \mathrm{m} \mathrm{Cu}{ }^{2+}$ under aerobic (light gray bars) and anaerobic (dark gray bars) con ditions were tested for their chaperone activity. Get3 oxidized aerobically with $2 \mathrm{mM} \mathrm{H}_{2} \mathrm{O}_{2} / 50 \mu \mathrm{M}$ $\mathrm{Cu}^{2+}$ served as control. 3-6 replicates were performed and the $\mathrm{SE}$ is shown.

kinetics (Kachur et al 1999) similar to the ones that I observed in the copper induced activation process of Get3 (Figure 3.1.3, black triangles). To test whether Get3's cysteines are indeed oxidized upon treatment with $\mathrm{H}_{2} \mathrm{O}_{2} / \mathrm{Cu}^{2+}$ or $\mathrm{Cu}^{2+}$, I monitored the thiol oxidation state using the Ellman's reagent (Riddles et al 1983). In parallel, I incubated Get3red under anaerobic conditions to test whether air oxidation is involved in $\mathrm{Cu}^{2+}$ mediated Get3 activation. In order to determine cysteine thiols, the protein was denatured in $6 \mathrm{M} \mathrm{Gdn}-\mathrm{HCl}$ prior to the incubation with Ellman's reagent. As expected, denatured Get3red incubated with the Ellman's reagent revealed the seven cysteines (Figure 3.1.6 A) that are present in yeast Get3. In contrast, the two fully chaperone-active species, Get3 treated with either $2 \mathrm{mM} \mathrm{H}_{2} \mathrm{O}_{2} / 50 \mu \mathrm{M}$ $\mathrm{Cu}^{2+}$ for $10 \mathrm{~min}$ (Get3ox) or with $50 \mu \mathrm{M} \mathrm{Cu}{ }^{2+}$ for less than $240 \mathrm{~min}$ (Get3Cu2+) under aerobic conditions revealed only about three reduced cysteines, 
suggesting the presence of four oxidized thiols (Figure 3.1.6 A). In agreement with our theory, Get3red treated with $50 \mu \mathrm{M} \mathrm{Cu}^{2+}$ under aerobic and anaerobic conditions behaved similar and showed about 3-4 reduced cysteines and no significant difference in chaperone activity (Figure 3.1.6 A and B). My data suggest the formation of two disulfide bonds in Get3ox, whose formation either precedes or parallels the activation of Get3 as ATP-independent chaperone. Extended incubation of proteins with oxidants can add additional modifications to various amino acid side chains beyond thiol oxidation and therefore alter protein function (Pattison \& Davies 2006, Reis et al 2011, Storkey et al 2014). Hence, we avoided copper oxidized Get3 and conducted all following experiments with $\mathrm{H}_{2} \mathrm{O}_{2} / \mathrm{Cu}^{2+}$ oxidized Get3 that turns fully chaperone active in less than $10 \mathrm{~min}$ (Figure 3.1.3, Get3ox, black circles).

The formation of four modified cysteines in Get3ox in vitro is consistent with the observation that at least four cysteines in yeast Get3 are important for cell survival (Figure 3.1.1 A), suggesting that the conserved cysteines (Cys 240/242/285/288) might be indeed the in vivo and in vitro targets for oxidants. In order to identify and characterize the four modified cysteines in more detail, we carried out mass spectrometry (MS) analysis of Get3ox. Therefore, we first tested the proteolytic stability of the two Get3 forms. $30 \mathrm{sec}$ of pepsin treatment in the presence of $1 \mathrm{M} \mathrm{Gdn}-\mathrm{HCl}$ was sufficient to fully digest Get3red into numerous fragments. Incubation of disulfide-bonded Get3ox with pepsin in the presence of $1 \mathrm{M} \mathrm{Gdn}-\mathrm{HCl}$ and $100 \mathrm{mM}$ of the strong thiol reductant tris(2-carboxyethyl)phosphine (TCEP), showed a similar digestion pattern (Figure 3.1.7, right panel), indicating that oxidation does not cause irreversible protein modifications. In contrast, pepsin digest of Get3ox in the absence of reducing agents revealed striking differences, with a nearabsence of proteolytic cleavage in the regions surrounding both pairs of conserved cysteines as well as the non-conserved Cys 317 (Figure 3.1.7, left panel). In agreement with our hypothesis that the conserved cysteines are involved in disulfide bond formation, these findings suggest that disulfide bond formation prevents pepsin from accessing its target sites (Figure 3.1.7 A, red arrow). We manually inspected the MS spectra for Get3ox (+/- TCEP) and identified two additional peptides, which harbor the conserved 
A A Quench: $1 \mathrm{M} \mathrm{GuHCl} \quad \ldots--\rightarrow$ Quench: $1 \mathrm{M} \mathrm{GuHCl} / 100 \mathrm{mM}$ TCEP
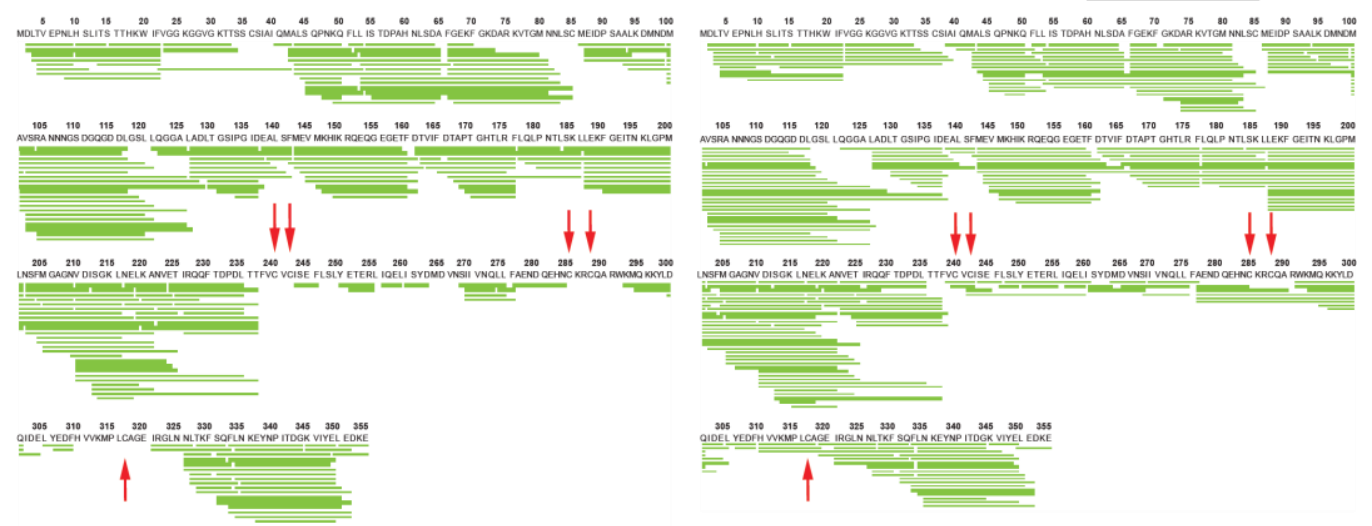

B
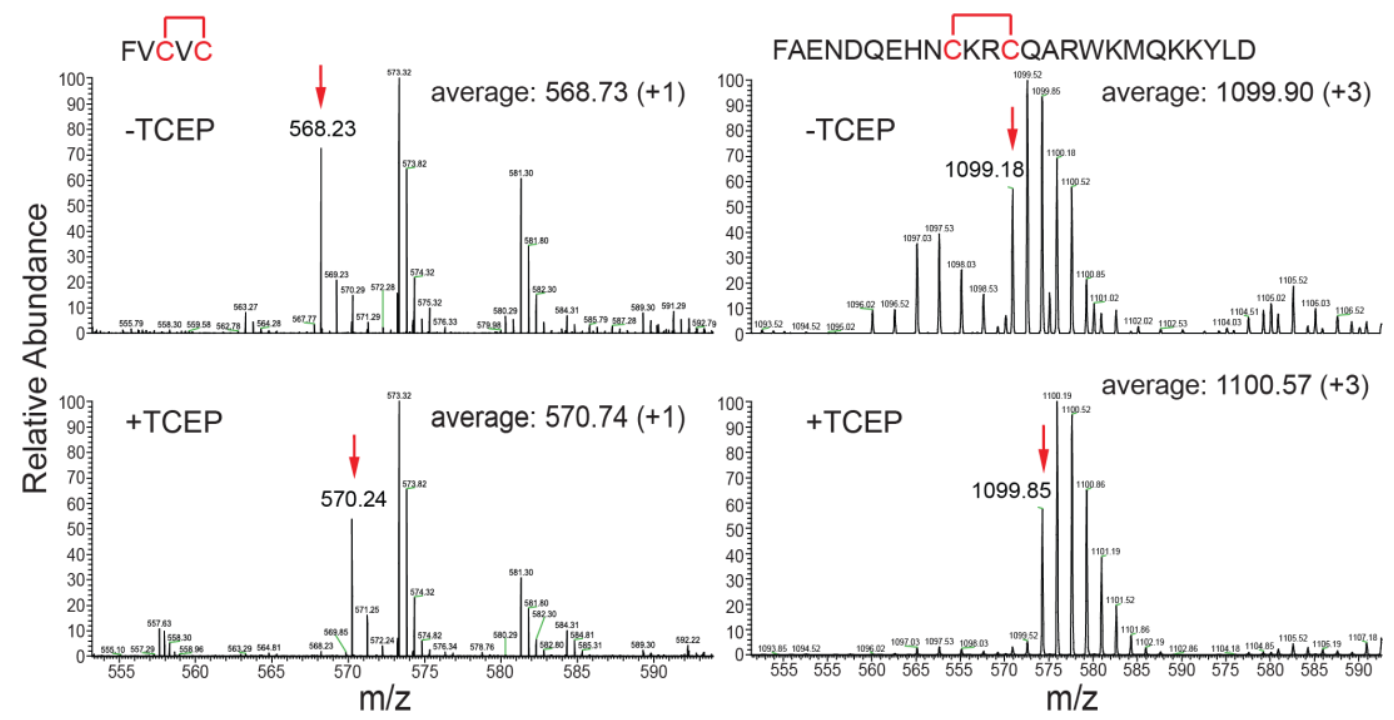

Figure 3.1. 7: Oxidation mediated disulfide bond formation in Get3

(A) Pepsin digest of $\mathrm{Get}_{\text {ox }}$ quenched with $1 \mathrm{M}$ guanidine hydrochloride ( $\mathrm{Gdn}-\mathrm{HCl}$ ) in the absence or presence of the reducing agent TCEP revealed differences in the patterns of proteolytic cleavage surrounding the two pairs of conserved cysteines (red arrows). Quenching of Get $3_{\text {ox }}$ in the presence of 100 mM TCEP reconstituted the cleavage pattem of Get3 $3_{\text {red }}$ shown in Figure 3.1.18 (B) Mass spectrometry (MS) spectra for Get3 peptides containing the CVC and CXYC-motif digested with pepsin in the presence or absence of $100 \mathrm{mM}$ TCEP. The calculated mass of the first monoisotopic peaks (red arrow) in both peptides shows a mass increase of $\sim 2$ Da upon addition of the reducing agent TCEP, consistent with the reduction of one disulfide bond. Pepsin digest and MS analysis were carried out by LiS (University of California).

cysteine pairs (CVC and CXYC) in their oxidized, disulfide-bonded state. The calculated mass of the first monoisotopic peak in both peptides showed a mass increase of 2 DA upon addition of the reducing agent TCEP, consistent with the reduction of one disulfide bond. (Figure 3.1.7 B, 
A

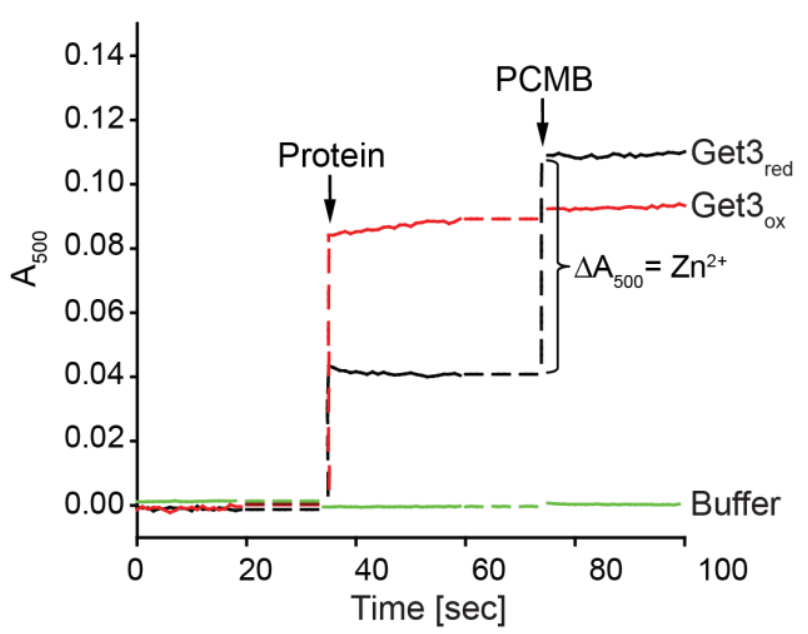

B

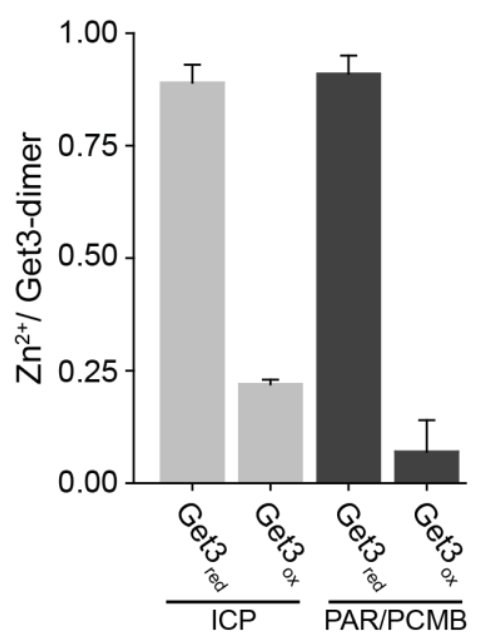

Figure 3.1. 8: Oxidation mediated zinc release in Get3

(A) The absorbance of PAR containing buffer was monitore dat $500 \mathrm{~nm}$ and either Get $3_{\text {red }}$ (black trace) or Get3 $3_{\text {ox }}$ (red trace) were added after $\sim 30 \mathrm{sec}$ (protein). Increased absorbance reflects the reaction of PAR with surface bound metals on the protein. Subsequent addition of PCMB elicited complete release of cysteine coordinated zinc indicated by an increased absorbance. (B) The precise amount of zinc associated with reduced and oxidized Get 3 was determined by ICP analysis (light gray bars). The amount of released zinc from the protein samples tested in the PAR/PCMB assay in A was calculated based on the increase in absorbance upon PCMB addition from a calibration curve for defined concentrations of zinc (dark gray bars). At least 3 replicates were performed and the SE is shown.

red arrow). This result indicates that the four conserved cysteines are involved in two intramolecular disulfide bonds, connecting the next neighbor cysteines with each other.

In reduced Get3, the two cysteine thiols of the C-X-Y-C motif contribute to dimer formation via zinc coordination (Mateja et al 2009). To test if zinc is still present when Get3 is oxidized, we performed ICP analysis on reduced and oxidized Get3. For the reduced form of Get3, we detected the expected 0.9 zinc equivalents per Get3 dimer. In contrast, oxidized Get3 only contained about 0.2 equivalents of zinc (Figure 3.1.8 B, light gray bars). To discriminate between high affinity cysteine-coordinated and low affinity surface-bound zinc in Get3, we used the 4-(2-pyridylazo)resorcinol/ parachloromercuribenzoic acid (PAR/PCMB) assay (llbert et al 2007, Jakob et al 2000). Complex formation of PAR with divalent cations such as $\mathrm{Fe}^{2+}, \mathrm{Ni}^{2+}$ or $\mathrm{Zn}^{2+}$ can be 

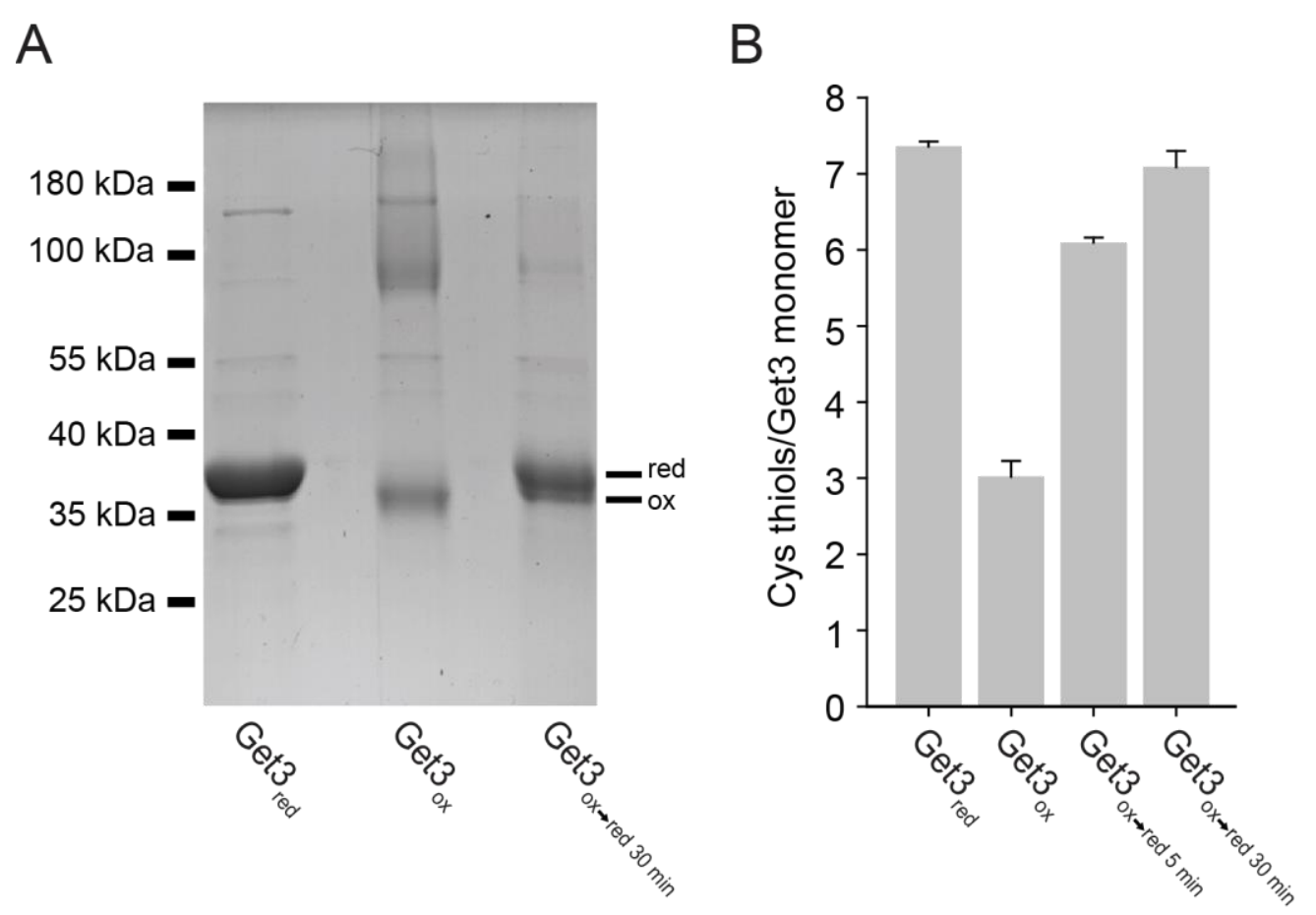

Figure 3.1. 9: Oxidation of Get3's thiols is fully reversible

(A) Analysis of Get $3_{\text {red }}$, Get $3_{\text {ox }}$ and Get $3_{\text {ox }}$ treated with $5 \mathrm{mM}$ dithiothreitol (DTT) at $30^{\circ} \mathrm{C}$ for 30 min on nonreducing SDS-PAGE. (B) Get $3_{\text {ox }}$ was treated with $5 \mathrm{mM} \mathrm{DTT}$ at $30^{\circ} \mathrm{C}$ for $5 \mathrm{~min}$ and $30 \mathrm{~min}$ respectively. The reductant was removed by gel filtration and the number of cysteine thiols was determined using the Ellman's assay as previously described. Get $3_{\text {red }}$ and Get $3_{\text {ox }}$ served as controls. 3 replicates were performed and the SE is shown.

monitored at a wavelength of $500 \mathrm{~nm}$ (A500). Get3red or Get3ox added to the PAR containing buffer increased the $\mathrm{A} 500$ signal (Figure 3.1.8 A, protein), indicating that PAR reacts with loosely surface bound metals. When PCMB, which forms mercaptide bonds with cysteines, was added to the mixture, $\sim 1$ zinc equivalent per Get3red dimer was released consistent with the results obtained from the ICP analysis (Figure 3.1.8 A and B, compare light and dark gray bars). No increase in PAR signal was observed upon addition of PCMB to Get3ox (Figure 3.1.8 A and B), suggesting that Get3red released its cysteine-coordinated zinc upon oxidation. Notably, Get3ox shows a higher content of surface bound metals, which suggests that the zinc that was released upon oxidation may remain attached to the protein's surface. From these experiments we concluded that Get3's activation as an ATPindependent chaperone involves thiol oxidation and concomitant 
release of zinc.

Next, I analyzed reduced and oxidized Get3 on nonreducing SDS-PAGE. Get3red showed the expected monomeric migration behavior, whereas Get3ox monomer migrated slightly faster, consistent with the presence of one or more intramolecular disulfide bonds (Figure 3.1.9). In addition, however, the Get3ox preparation revealed the presence of dimers and higher oligomers, suggesting the formation of intermolecular disulfide bonds. Addition of reducing agent to oxidized Get3 recovered the monomeric migration behavior observed for Get3red on SDS-PAGE (Figure 3.1.9, Get3ox $\rightarrow$ red) indicating that the higher oligomers were indeed mediated via disulfide bonds. The absence of proteolytic cleavage in the regions surrounding Cys 317 in Get3ox (Figure 3.1.7, red arrow in left panel) may suggest the formation of an additional intermolecular disulfide bond. To obtain clear results on the disulfide connectivities in Get3ox, quantitative MS analysis needs to be performed.

\subsubsection{Activation of Get3's chaperone function is a fully reversible process in vitro}

Reversibility is a major aspect of every posttranslational regulation event. To test whether the activation of Get3's chaperone function and the inactivation of Get3's ATPase activity are reversible processes in vitro, I first oxidized and activated Get3red for 4 min with $\mathrm{H}_{2} \mathrm{O}_{2} / \mathrm{Cu}^{2+}$, which is the minimal incubation time to reach full chaperone activity upon treatment (Figure 3.1.3, black circles). After removal of the oxidants by gel filtration, I added DTT and $\mathrm{Zn}$ to reduce any reversible thiol modifications and to reconstitute the zinc binding site. I found that incubation with $\mathrm{DTT}$ alone rapidly re-reduced the cysteines in Get3ox. After only 5 min of DTT treatment, six cysteines were detectable and within 30 min of incubation all seven cysteines thiols were reduced (Figure 3.1.9. B, Get3ox $\rightarrow$ red 5/30 min). Despite the rapid rereduction of the cysteines, however, not even a 6-hour incubation with DTT and zinc at $30^{\circ} \mathrm{C}$ caused any inactivation of Get3's chaperone function (Figure 3.1.10 A). These results were highly reminiscent of oxidized Hsp33, whose inactivation was found to be very slow even though the reduction of 
A

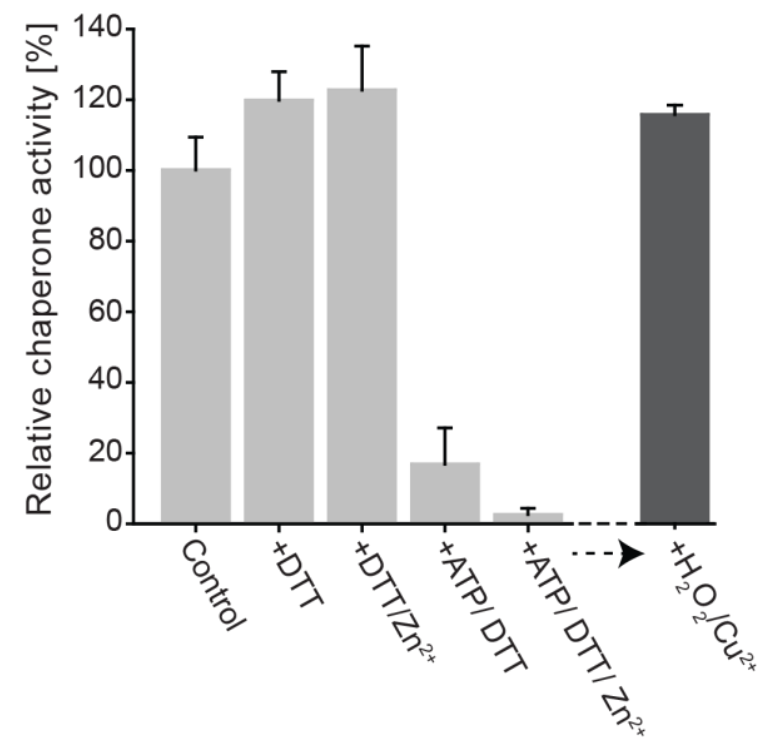

B

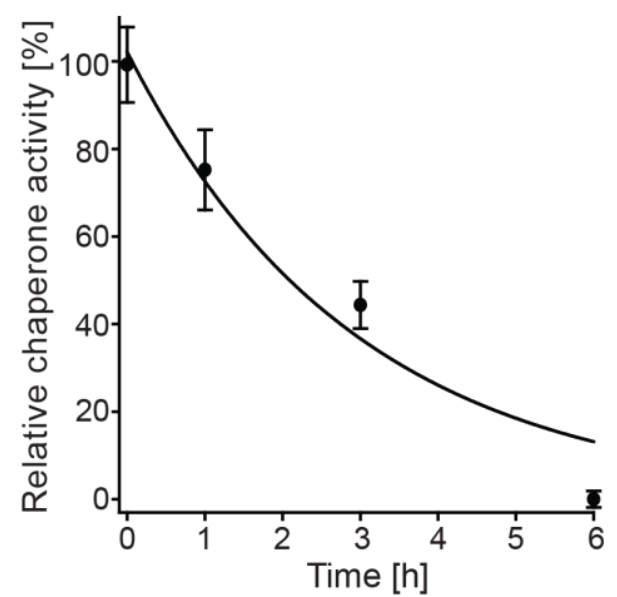

Figure 3.1. 10: Activation of Get3's chaperone function is a reversible process

(A) $5 \mu \mathrm{M}$ of chaperone-active wild-type Get $_{\text {ox }}$ (control) was incubated in the presence of $5 \mathrm{mM}$ DTT alone or with various combinations of $5 \mathrm{mM}$ DTT, $5 \mu \mathrm{M}$ zinc and $2 \mathrm{mM}$ MgATP for $6 \mathrm{~h}$ at $30^{\circ} \mathrm{C}$. The reductants were removed via gel filtration and the various Get3 preparations were tested for their ability to prevent the aggregation of chemically denatured CS as outlined in Figure 3.1.2. Subsequently the chaperone-inactivated $\mathrm{Get}_{\text {ox } \rightarrow \text { red }}$ (+ATP/DTT/Zn) was incubated with $2 \mathrm{mM} \mathrm{H}_{2} \mathrm{O}_{2}$ and $50 \mathrm{mM} \mathrm{Cu}^{2+}$ for $10 \mathrm{~min}$ and assayed for its chaperone function as described, revealing that Get3 can undergo multiple rounds of oxidation and reduction processes. (B) Get $3_{\text {ox }}$ was incubated in the presence of $5 \mathrm{mM}$ DTT, $5 \mu \mathrm{M}$ zinc and $2 \mathrm{mM} \mathrm{MgATP}$ for $6 \mathrm{~h}$ at $30^{\circ} \mathrm{C}$. At indicated time points the chaperone activity was tested as described in Figure 3.1.3. The signal of aggregated CS in the absence of added chaperones was set to $0 \%$ chaperone activity, while the signal of Get $3_{\text {ox }}$ immediately assayed upon addition of the reductants was set to $100 \%$. The data points were fitted with SigmaPlot to an exponential decay function using a single exponential fit and the halftime was calculated to be $\sim 2 \mathrm{~h}$ (see methods part for details). At least 3-6 replicates were performed and the SE is shown.

its disulfide bonds occurred very rapidly (Hoffmann et al 2004). Hence, we reasoned that similar to Hsp33, Get3 might undergo conformational rearrangements triggered by cysteine oxidation and oligomerization. Therefore, upon re-reduction the re-organization of the structural changes might become rate-limiting in the inactivation process of oxidized Get3.

To visualize the secondary structure of Get3red and Get3ox, we therefore conducted far-UV-circular dichroism (CD) spectroscopy experiments. The 

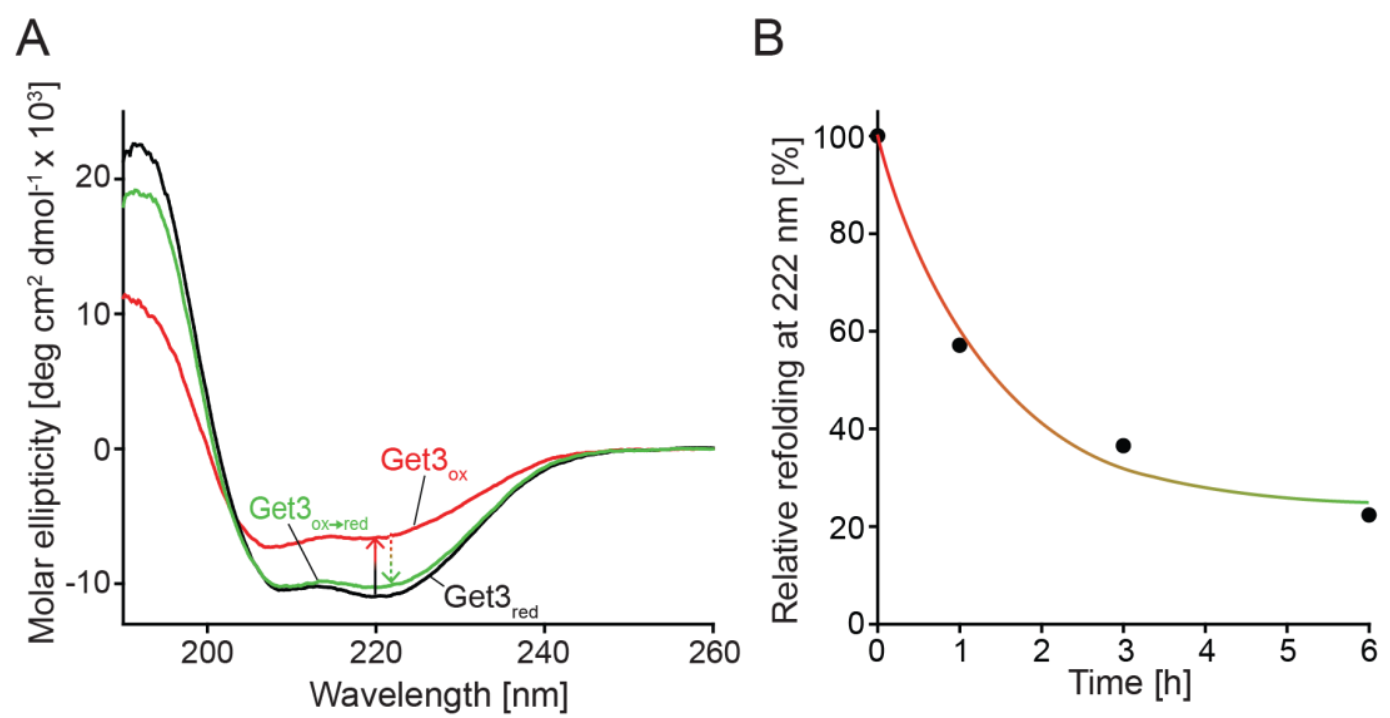

Figure 3.1. 11: Oxidized Get3 undergoes reversible structural rearrangements

(A) Analysis of Get3's secondary structure using far-UV circular dichroism (CD) spectroscopy. Get $3_{\text {red }}$ (black trace), Get $3_{\text {ox }}$ (red trace) and Get $3_{\text {ox } \rightarrow \text { red }}$ (green trace) were freshly prepared according to Figure 3.1.10 A and analyzed directly. (B) Recovery of Get $_{\text {ox }}$ structure upon addition of $5 \mathrm{mM}$ DTT, $5 \mu \mathrm{M}$ zinc and $2 \mathrm{mM} \mathrm{MgATP}$. At indicated time points the reductants were removed via gel filtration and changes in the molar ellipticity were recorded at $222 \mathrm{~nm}$ using a CD spectropolarimeter. Changes were

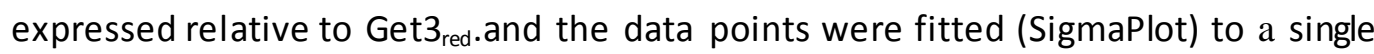
exponential decay fit, the halftime was calculated to be $\sim 1 \mathrm{~h}$.

CD spectra of reduced Get3 confirmed the predominantly alpha-helical structure of Get3, revealing two minima at 208/222 nm and a maximum at $190 \mathrm{~nm}$ (Figure 3.1.11 A, black trace). Analysis of the CD spectra of oxidized Get3 revealed major changes, indicative of extensive conformational rearrangements. We observed a substantial loss in a-helical content shown by the decreased signal at $\sim 190 \mathrm{~nm}$ and the accumulation of random-coil structure reflected in the increase at $\sim 220 \mathrm{~nm}$ (Figure 3.1.11 A, red trace). In addition, we incubated the protein with bis(4-anilino-5napththalenesulfonic acid (bis-ANS), a fluorescent sensor molecule of hydrophobic surfaces. Subsequent measurements of the fluorescence intensity revealed a much higher signal for Get3ox compared to Get3red. (Figure 3.1.12 A, black and red trace). These results indicate that the structural rearrangements in Get3ox were accompanied by the exposure of 
A

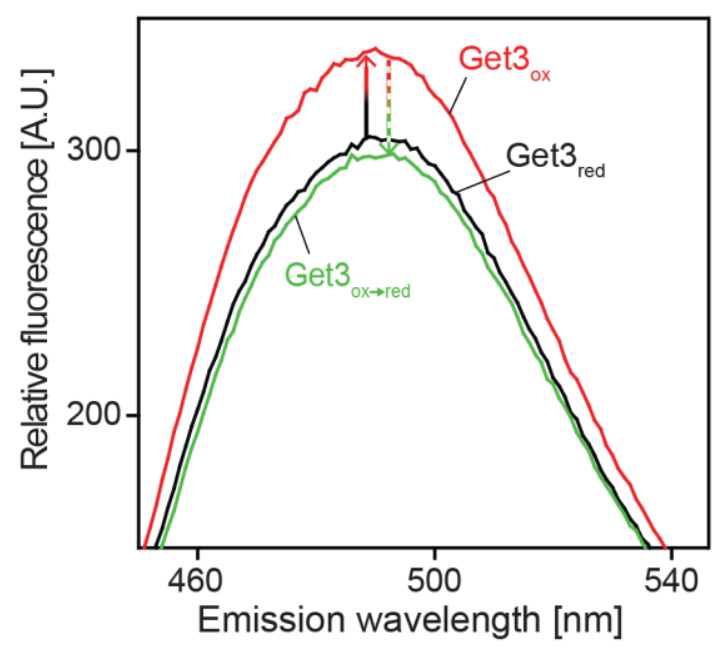

B

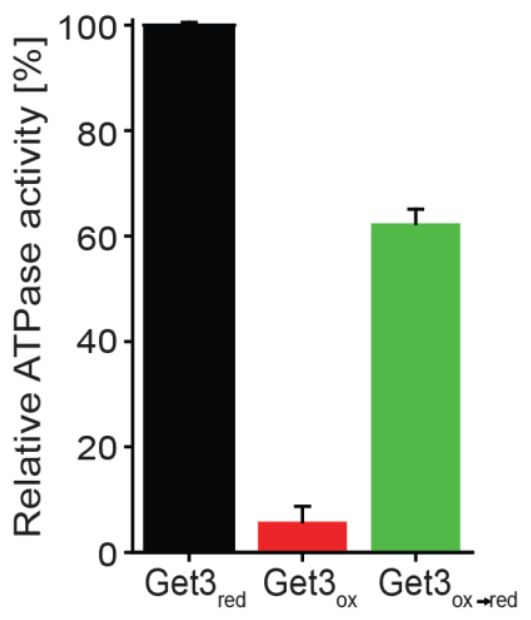

Figure 3.1. 12: Oxidation reversibly alters Get3's protein characteristics

(A) Measurements of Get3's surface hydrophobicity with bis-ANS fluorescence spectroscopy. Get $3_{\text {red }}$ (black trace), Get3 $3_{\text {ox }}$ (red trace) and Get $3_{\text {ox } \rightarrow \text { red }}$ (green trace) were prepared according to Figure 3.1.10 A and analyzed subsequently. (B) The ATPase activity of $\mathrm{Get} 3_{\mathrm{ox}}$ and $\mathrm{Get}_{\mathrm{ox} \rightarrow \mathrm{red}}$ relative to untreated Get3 ${ }_{\text {red }}$ is shown. At least 3 replicates were performed and the SE is shown. Freshly prepared Get $3_{\text {red }}$ (black trace), Get $_{\text {ox }}$ (red trace) and Get3 $3_{\text {ox } \rightarrow \text { red }}$ (green trace) were used.

hydrophobic surfaces, a hallmark of active chaperones.

Since Get3's ATP-binding site is located in close vicinity to one of the two highly conserved cysteine pairs that undergo disulfide bond formation in Get3ox (Figure 1.7, CVC motif), we reasoned that Mg-ATP binding might stabilize the reduced conformation and therefore accelerate the refolding to the reduced conformation and hence the inactivation of Get3's chaperone function. Indeed, full inactivation of Get3ox was achieved when MgATP was added to the DTT/Zn mixture (Figure 3.1.10 $\mathrm{A}$ and $\mathrm{B}$ ). These results have physiological significance since restoration of cellular ATP levels indicates a cell's return to pre-stress conditions. Consistent with these results, analysis of the secondary structure of Get3 revealed an almost complete reversal of the structural rearrangements, concomitant with a decrease in surface hydrophobicity in Get3ox upon its incubation in MgATP, DTT and $\mathrm{Zn}^{2+}$ (Figure 3.1.11 A and Figure 3.1.12 A, compare red and green traces). It is of note that the recovery of Get3's secondary structure slightly precedes 


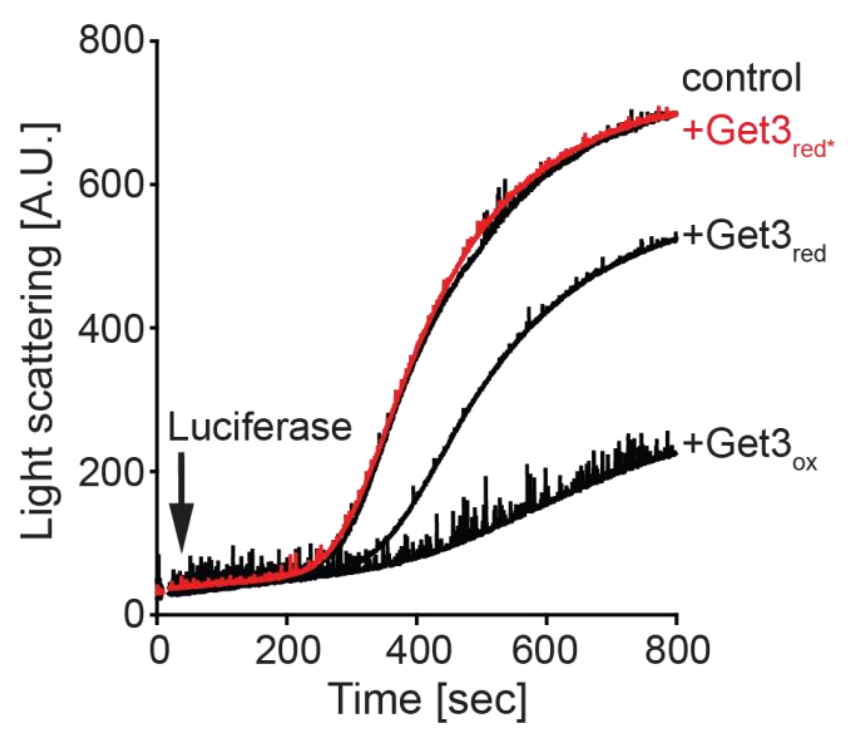

Figure 3.1. 13: Fully inactivated Get $3_{\text {red }}$ shows no chaperone activity Get3 treated with $5 \mathrm{mM} \mathrm{DTT}$ at $30^{\circ} \mathrm{C}$ for $1 \mathrm{~h}\left(\mathrm{Get}_{\mathrm{red}}\right)$ and Get 3 diluted to a concentration of $5 \mu \mathrm{M}$, incubated in the presence of $5 \mathrm{mM}$ DTT, $0.5 \mathrm{mM} \mathrm{MgATP}$ and $5 \mu \mathrm{M} \mathrm{ZnCl}_{2}$ (Get3 ${ }_{\text {red* }}$, red trace) were tested for their influence on the light scattering of $100 \mathrm{nM}$ thermally unfolding luciferase at $43^{\circ} \mathrm{C}$ (Get3:Iuciferase ratio 1:1). Reductants were removed by gel filtration prior to the assay as described in the material and methods part. Light scattering of aggregating luciferase measured in the absence of added chaperones (control) and in the presence of Get $3_{\text {ox }}\left(+G e t 3_{o x}\right)$ is shown.

the inactivation of its chaperone activity (Figure 3.1.10 $\mathrm{B}$ and Figure $3.1 .11 \mathrm{~B}$ ). Subsequent ATPase activity measurements revealed that re-reduced Get3 regained its ATPase activity (Figure 3.1.12 B, green bar). My results therefore demonstrate that Get3's oxidative activation as an ATPindependent chaperone is a fully reversible process in vitro, allowing Get3 to undergo multiple rounds of oxidative activation as molecular chaperone (Figure 3.1.10 A, $+\mathrm{H}_{2} \mathrm{O}_{2} / \mathrm{Cu}^{2+}$ ).

When we previously tested reduced and oxidized Get3 for their chaperone activity, we observed that Get3red exhibited considerable chaperone activity in the thermal and chemical aggregation assay conducted with luciferase (Figure 3.1.2 C). We reasoned at that point that reduced Get3 was potentially destabilized by high temperatures and therefore might unfold, leading to increased chaperone activity. However, when I now applied our established inactivation protocol (i.e., incubation with $0.5 \mathrm{mM}$ 
A

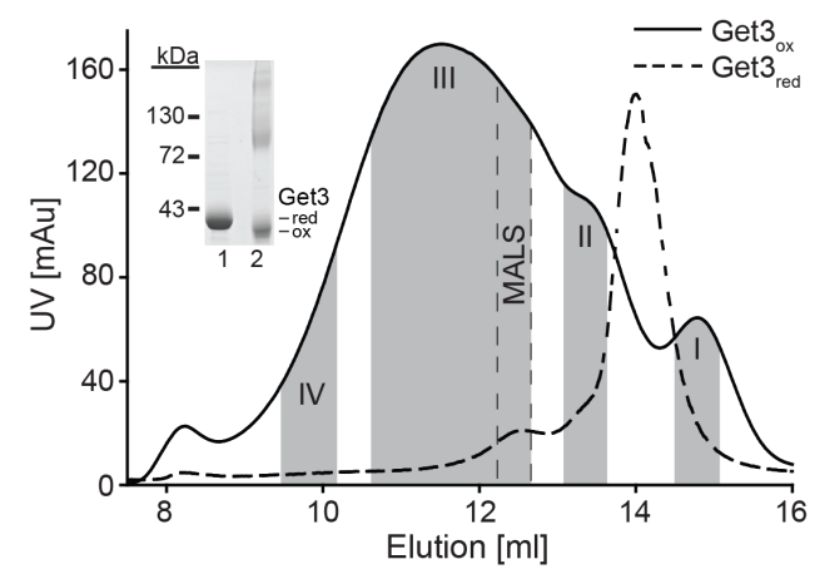

B

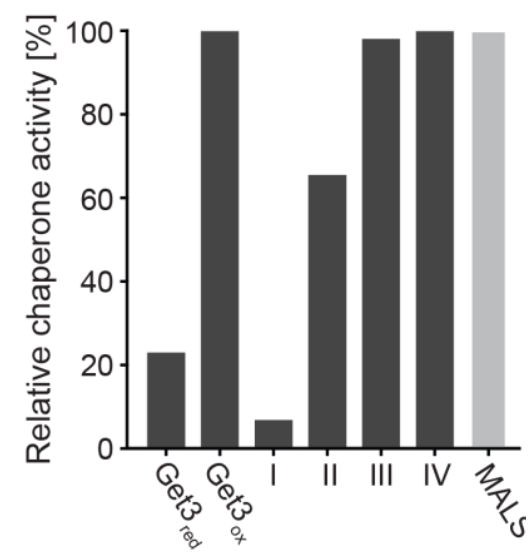

Figure 3.1. 14: Identification of smallest chaperone-active Get $3_{\text {ox }}$ unit

(A) Get $3_{\text {red }}$ (dashed line) and Get $3_{\text {ox }}$ (solid line) were analyzed on size exclusion chromatography (Superdex 200 10/300 GL column). Indicated peak fractions (gray shaded areas) containing various oligomeric states of Get $3_{\text {ox }}$ were collected (fractions IV) and (B) subsequently analyzed for their ability to prevent the aggregation of chemically denatured CS (dark gray bars) as described in Figure 3.1.2. A second batch of Get $3_{\text {ox }}$ was loaded on size exclusion chromatography and a smaller fraction indicated as MALS in A (dashed bar) was tested for its chaperone activity (B, light gray bar). Chaperone activity of Get3 $3_{\text {ox }}$ was set to $100 \%$. Inset: analysis of Get $3_{\text {red }}$ (lane 1) and Get3 $3_{\text {ox }}$ (lane 2) on nonreducing SDS-PAGE.

MgATP, $5 \mathrm{mM} \mathrm{DTT}$ and $5 \mu \mathrm{M} \mathrm{ZnCl}$ ) ) on purified Get3, I obtained close to fully inactive Get3 (Figure 3.1.13). These results indicate that Get3 purified under reducing conditions was presumably partially unfolded and hence not fully inactivated as a general chaperone upon incubation with DTT only.

\subsubsection{The minimal unit of chaperone-active Get3 is the oxidized tetramer}

Previous analysis of oxidized Get3 on nonreducing SDS-PAGE indicated the additional presence of Get3 dimers and higher oligomers (Figure 3.1.9). To further characterize the higher oligomeric species, I separated the Get3ox preparation using a size exclusion chromatography. I found that the highest oligomeric species eluted with a molecular weight corresponding to a $\sim 400 \mathrm{kDa}$ protein, indicating a 10-fold increase in size compared to Get3ox monomers that eluted at $\sim 40 \mathrm{kDa}$ (Figure 3.1.14 A, compare shaded areas 


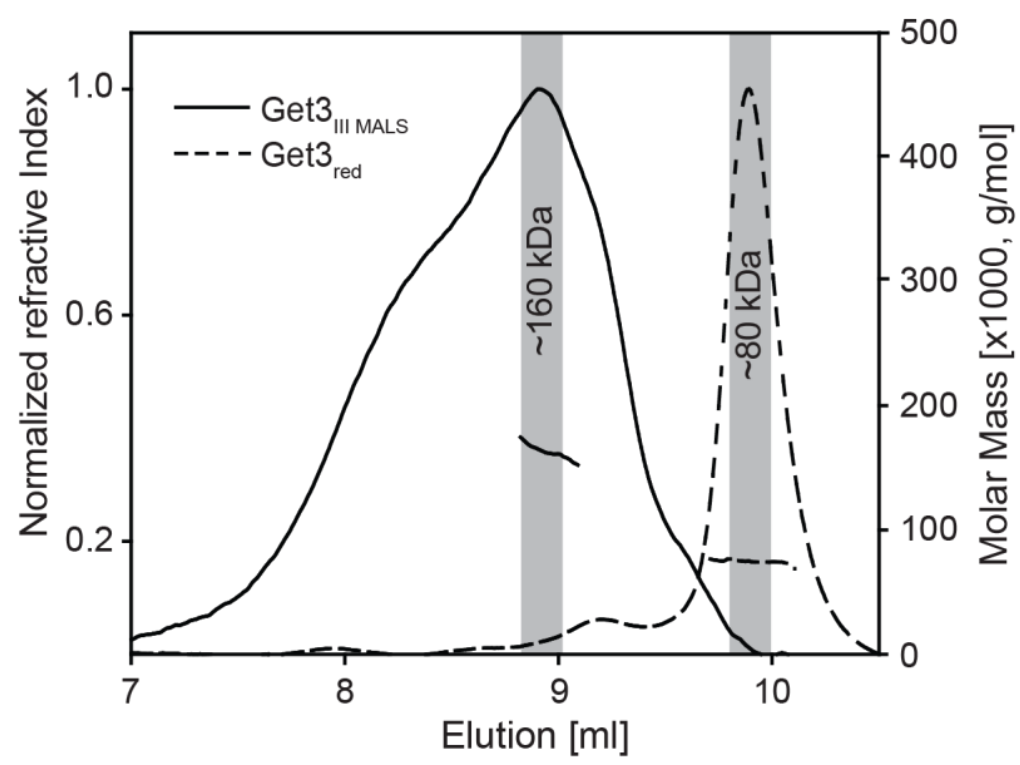

Figure 3.1. 15: The smallest chaperone-active $\mathrm{Get}_{\text {ox }}$ unit is a tetramer

Get3 $3_{\text {red }}$ and the Get $3_{\text {ox }}$ sub-fraction of fraction III (indicated as MALS in Figure 3.1.14) were analyzed by SEC-MALS and calculated to be approximately $80 \mathrm{kDa}$ and $160 \mathrm{kDa}$, respectively. This sizes are consistent with a Get $3_{\text {red }}$ dimerand a Get $3_{\text {ox }}$ tetramer. Areas shaded in light gray were latter analyzed on negative stain electron microscopy (EM).

IV and I). To investigate whether oligomerization correlates with the chaperone function of Get3ox, I collected individual peak fractions and subsequently determined their respective chaperone activity (Figure 3.1.14 A, shaded areas I-IV). Oligomeric species of Get3ox (fractions III and IV) exhibited the highest specific chaperone activity, whereas fractions harboring species that eluted at the same time as the reduced Get3 dimer showed only negligible chaperone activity (Figure 3.1.14 B). These results are consistent with findings on other chaperones, such as Leishmania peroxiredoxin or human Hsp27, which both undergo oligomerization processes to become chaperone active (Rogalla et al 1999, Teixeira et al 2015).

To test the stability of oxidized Get3's oligomers and to identify the smallest fully chaperone active unit, I collected smaller aliquots within fraction III (Figure 3.1.14 A, shaded area III). Next, I re-chromatographed each of them on a second size exclusion column connected to a static light scattering instrument for molecular weight determination (SEC-MALS) and tested their 


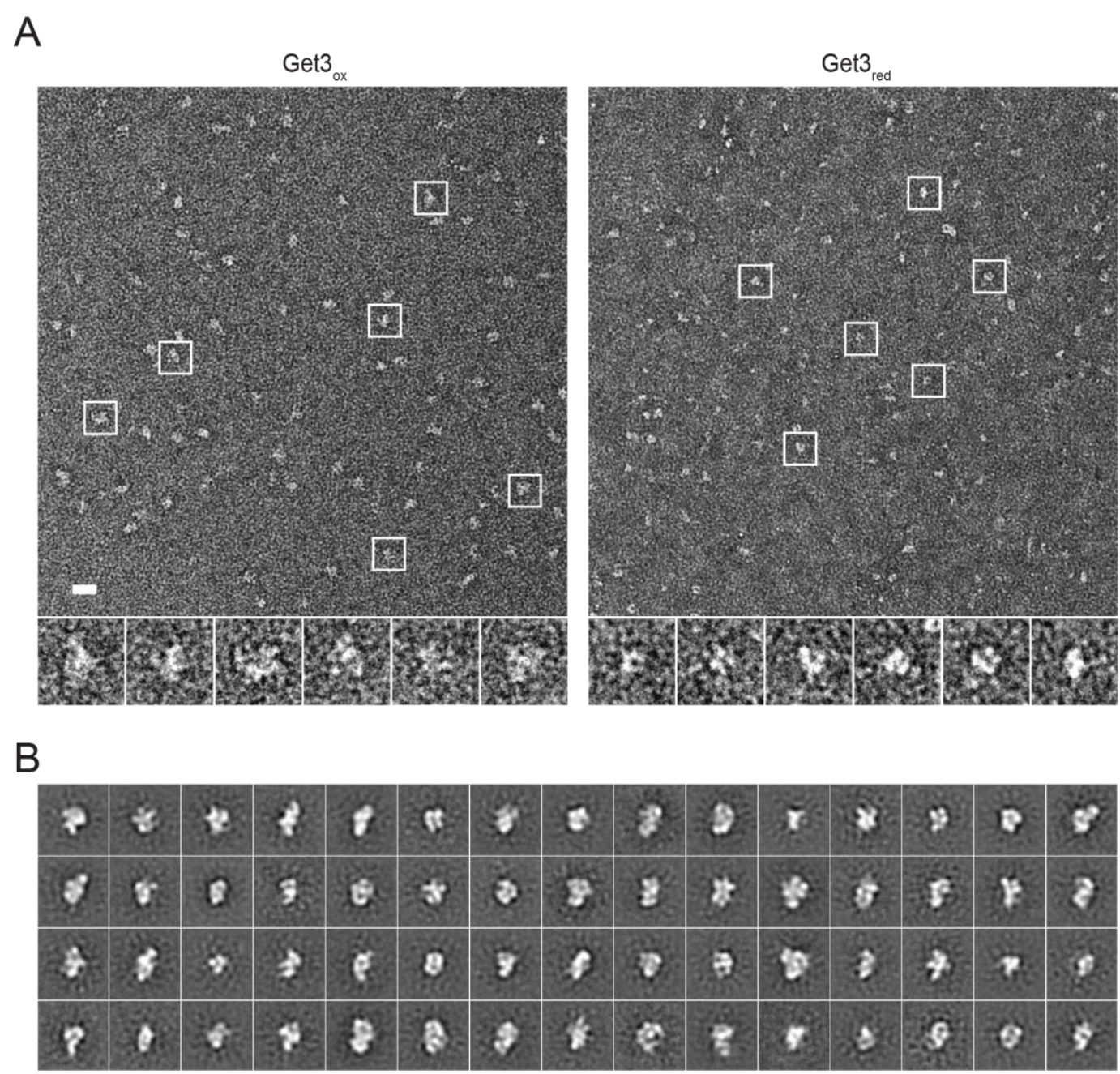

Figure 3.1. 16: Get3 oligomers imaged on negative-stain EM grids

(A) Peak fractions of SEC-MALS elution for the Get $3_{\text {ox }}$ tetramer and Get $3_{\text {red }}$ were collected (Figure 3.1.15, light gray shaded areas) and analyzed by negative-stain electron microscopy (EM). Representative micrograph images of selected Get3 $3_{\text {ox }}$ (left panel) and Get3 $3_{\text {red }}$ (right panel) particles are shown. Scale bar equals $200 \AA$ and the box size is $260 \AA$. (B) Complete set of reference-free class averages for Get $3_{\text {ox }}$ tetramer, generated using SPIDER (Frank et al 1996). Most of the imaging and all analysis were carried out by Gates $S$ (Southworth Laboratory, University of Michigan).

chaperone activity in parallel. The smallest chaperone-active Get3ox unit found within fraction III (Figure 3.1.14 A and B, MALS) eluted in the SECMALS run as a broad single peak with an average molecular weight of about $160 \mathrm{kDa}$ (Figure 3.1.15, gray shaded area for Get3III MALs run), suggestive of a Get3 tetramer. These results indicate that oxidized Get3 consists of stable oligomeric complexes in which a Get3 tetrameric form is likely to represent the minimal chaperone-active arrangement. Of note, the Get3III MALS elution 
revealed a shoulder, indicating the presence of some larger oligomers in this fraction as well. As shown in Figure 3.1.15, the reduced Get3 sample eluted with an average molecular weight of $80 \mathrm{kDa}$, consistent with the formation of a Get3 dimer under these conditions.

Next, we conducted negative-stain electron microscopy (EM) on the peak fraction following SEC-MALS elution (Figure 3.1.15, shaded area $160 \mathrm{kDa}$ and $80 \mathrm{kDa}$ ) to visualize the architecture of the Get3ox tetramer in comparison to the reduced Get3 dimer. Representative micrograph images of single Get3ox particles appear larger and in a different arrangement compared to images showing Get3red particles (Figure 3.1.16 A). We then collected single particle data sets from more than 100 micrographs and generated 2D reference-free projection averages for Get3ox. A closer look at the overall set of $2 \mathrm{D}$ averages revealed the additional presence of larger elongated complexes, suggestive of higher oligomeric states (Figure 3.1.16 B). However, the smaller more compact form of the complex clearly predominated (Figure 3.1.16 B). We therefore filtered out all the larger oligomeric states in the refinement process. The refined 2D reference-free projection averages for Get3ox show a globular arrangement with two defined lobes that form a ' $W$ ' shape in some views (Figure 3.1.17 A, RF Avg.). Notably, the arrangement we observed is different than the 2012 by Suloway et al. crystallized tetramer of the archaeal Get3 homologue, which forms an elongated 'dumbbell' like shape. In contrast to the oxidized Get3 tetramer, reduced dimeric Get3 clearly shows a small ' $U$ ' shaped arrangement (Figure 3.1.17 B, RF Avg.), which matches very well with $2 \mathrm{D}$ projections and corresponding $3 \mathrm{D}$ views of the published Get3 crystal structure ( $\mathrm{Hu}$ et al 2009) (Figure 3.1.17 B). A comparison of the two 2D projection images clearly reveals that Get3ox is larger than Get3red. Moreover, Get3ox contains two lobes that are consistent with two Get3 dimers connected in a tetrameric complex (Figure 3.1.17 A and $B$, Proj.).

In order to further characterize Get3's tetrameric architecture, a 3D reconstruction method described in great detail in the material and methods 
A

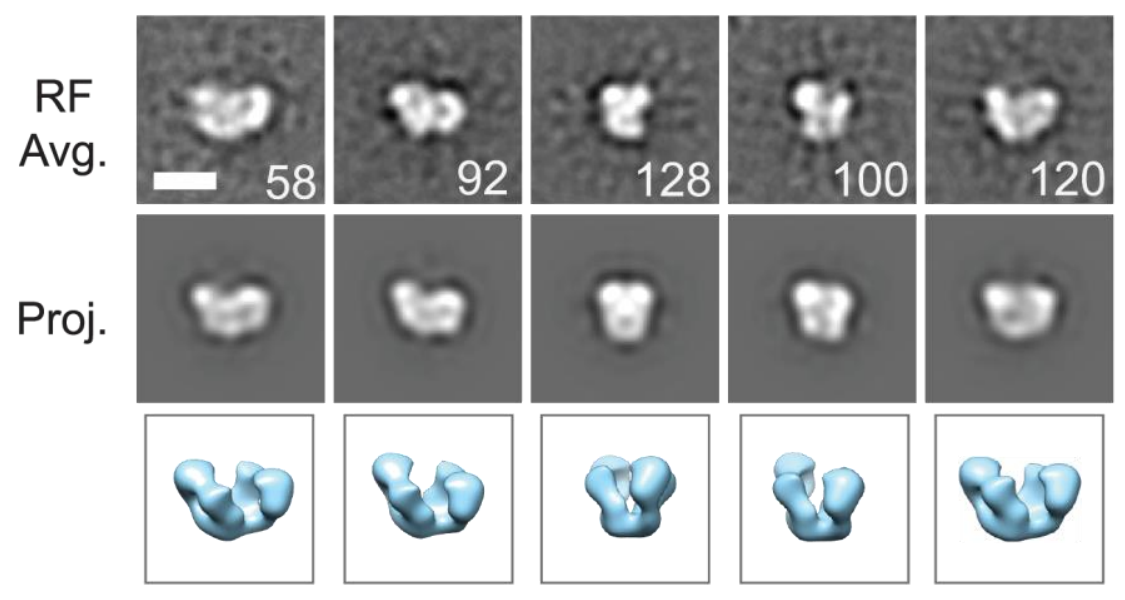

B

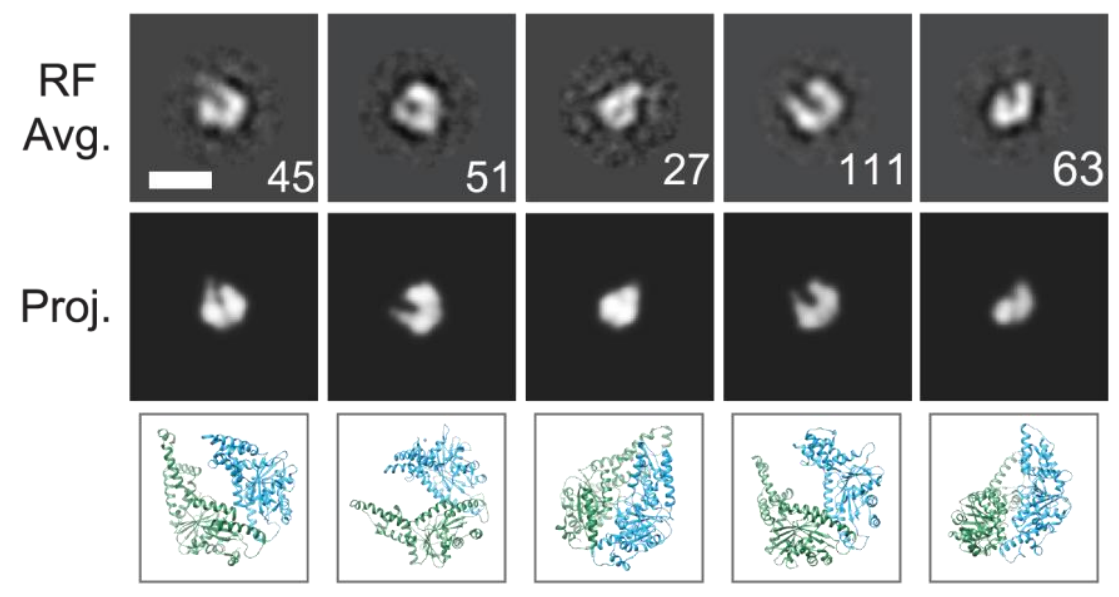

C

$120 \AA$

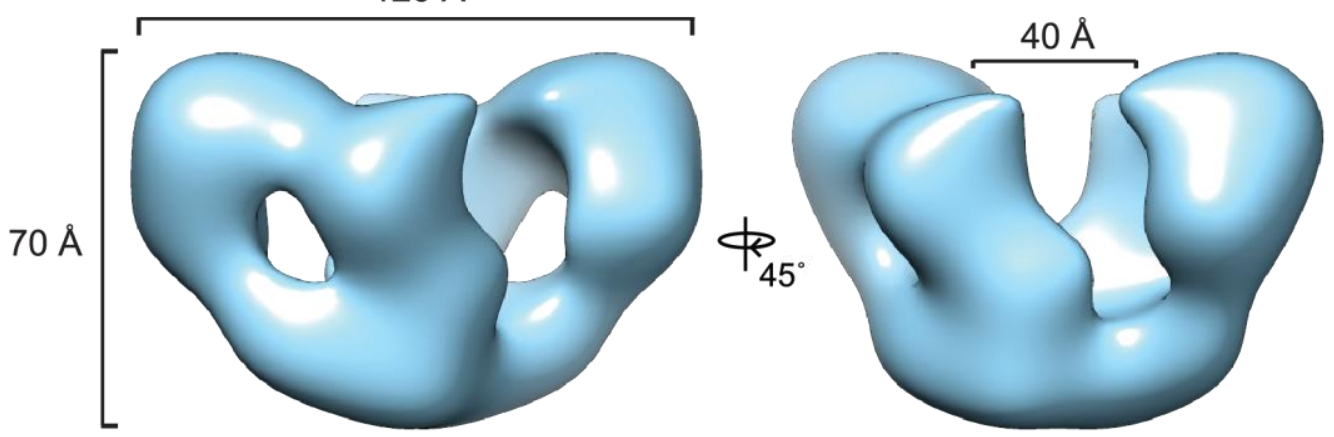

Figure 3.1. 17: 3D modeling of the Get3 ${ }_{\text {ox }}$ tetramer based on negative-stain EM data Distinct fractions of the SEC-MALS elution in Figure 3.1.15 (gray shaded area) were collected and used to determine negative-stain EM 2D reference-free class averages of (A) Get3 $3_{\text {ox }}$ and (B) Get3 $3_{\text {red }}$ using SPIDER (Frank et al 1996) (upper panel). Matching projections and corresponding images of the (A) Get $3_{\text {ox }} 3 \mathrm{D}$ model and (B) Get $3_{\text {red }}$ dimer crystal structure (Protein Data Bank ID: 3H84) are shown (Hu et al 2009) (middle and lower panel). The number of single particles is shown for each average and the scale bars equal $100 \AA$. (C) Final 3D model of the Get $3_{\text {ox }}$ tetramer complex, filtered to $19 \AA$. Dimensions are indicated in $\AA$. The analysis and 3D modeling were performed by Gates $\mathrm{S}$ (Southworth Laboratory, University of Michigan). 
part (chapter 2.10.3) was performed. The final reconstruction resulted in a 3D structure for tetrameric Get3ox with a resolution of $19 \AA$. The complex of 70 to $120 \AA$ consists of two oval-shaped domains connected along one side, forming a $40 \AA$ cleft between the two domains (Figure 3.1.17 C). An important control for $3 \mathrm{D}$ reconstructions is the back-projection of the final model into 2D projections, that should not differ from the initial 2D referencefree projection averages. Indeed, the reference-free averages agreed with the 2D back-projections of the model (Figure 3.1.17 A, compare RF Avg. and Proj.), supporting our 3D tetramer arrangement. Our approach to fit the crystal structures of the Get3red dimer into the tetrameric Get3ox model failed. However, the dimensions of each oval domain in our 3D model for the tetramer are consistent with the size of one dimer. In conclusion, the SEC-MALS runs and structural reconstruction from EM images revealed that the minimal chaperone-active unit of oxidized Get3 is a distinct tetrameric complex. Moreover, compared to previously characterized structures of the reduced Get3, the identified complex is clearly in a different conformation and arrangement.

\subsubsection{Oxidative activation of Get3 causes massive structural rearrangements}

Our previous spectroscopic studies suggested that Get3's oxidative activation process is accompanied by substantial structural rearrangements. To investigate these structural changes in more detail, we decided to conduct hydrogen-deuterium (H/D) exchange experiments of oxidized and reduced Get3. This state-of-the-art technique combines kinetic H/D exchange experiments with mass spectrometry, hence allowing us to monitor the structural changes in a protein with a resolution close to the amino acid level (Englander 2000, Kan et al 2013). Briefly, proteins of interest are incubated in deuterated buffer, which leads to the exchange of amide protons with "heavy" deuterium. At defined time points, the exchange reaction is quenched by a sudden shift to $\mathrm{pH}$ 2.5. Subsequently, the protein is incubated with pepsin in the presence of $1 \mathrm{M} \mathrm{Gdn-HCl}$, which allows efficient digest of the protein. Next, the resulting peptides are analyzed by 


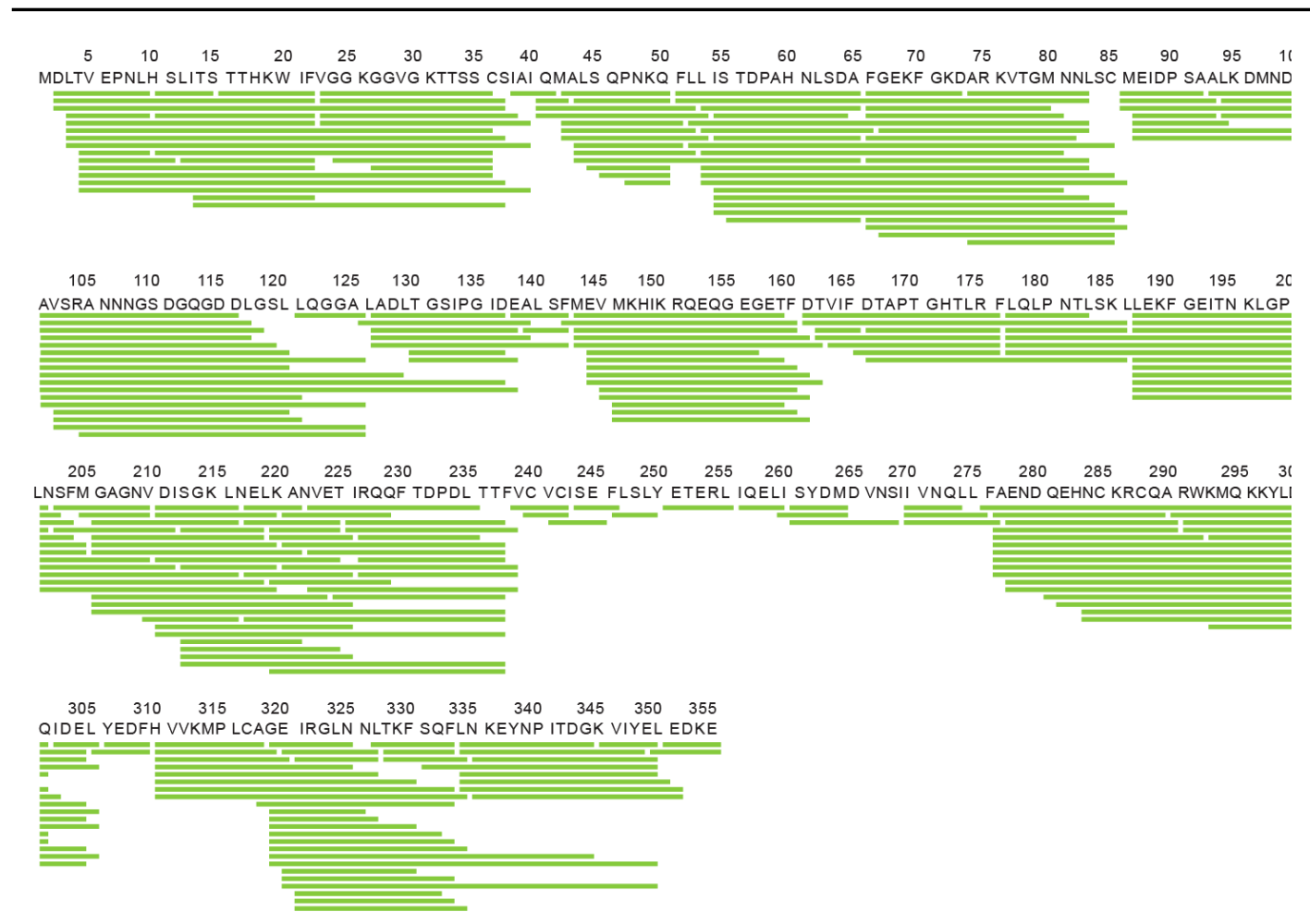

Figure 3.1. 18: Coverage map for pepsin digested Get3

Reduced Get3 was digest with pepsin and upon quenching with $1 \mathrm{M} \mathrm{Gdn-HCl}$ the digested protein was analyzed by MS. Resulting peptides are mapped on Get3's protein sequence. Pepsin digest of $\mathrm{Get}_{\text {ox }}$ quenched with $1 \mathrm{M} \mathrm{Gdn}-\mathrm{HCl}$ in the presence of $100 \mathrm{mM}$ TCEP was similar to the cleavage pattern of the Get $3_{\text {red }}$ digest (see Figure 3.1.7). Pepsin digest and MS analysis were carried out by Li S (University of California).

MS/MS, which allows a direct comparison of mass differences within single peptides. To determine the protein coverage, we first tested the proteolytic sensitivity of reduced Get3. We found that a $30 \mathrm{sec}$ treatment with pepsin in $1 \mathrm{M} \mathrm{Gdn} / \mathrm{HCl}$ was sufficient to digest Get3red into numerous, often overlapping fragments that covered $\sim 99.8 \%$ of the entire Get3 sequence (Figure 3.1.18). Pepsin digest of oxidized, disulfide bonded Get3 in the presence of $1 \mathrm{M} \mathrm{Gdn}-\mathrm{HCl}$ and $100 \mathrm{mM}$ TCEP resulted in a very similar pattern indicating that oxidation does not introduce irreversible modifications in Get3 (Figure 3.1.7 A). We then performed our $\mathrm{H} / \mathrm{D}$ exchange reaction with either Get3ox or Get3red, and subsequently conducted the $\mathrm{pH}$ quench and pepsin digest in the presence of TCEP. This method, which was established by 2010 by Zhang et al., avoids differences in pepsin coverage of oxidized and reduced proteins, and hence enabled us to directly compare the mass 


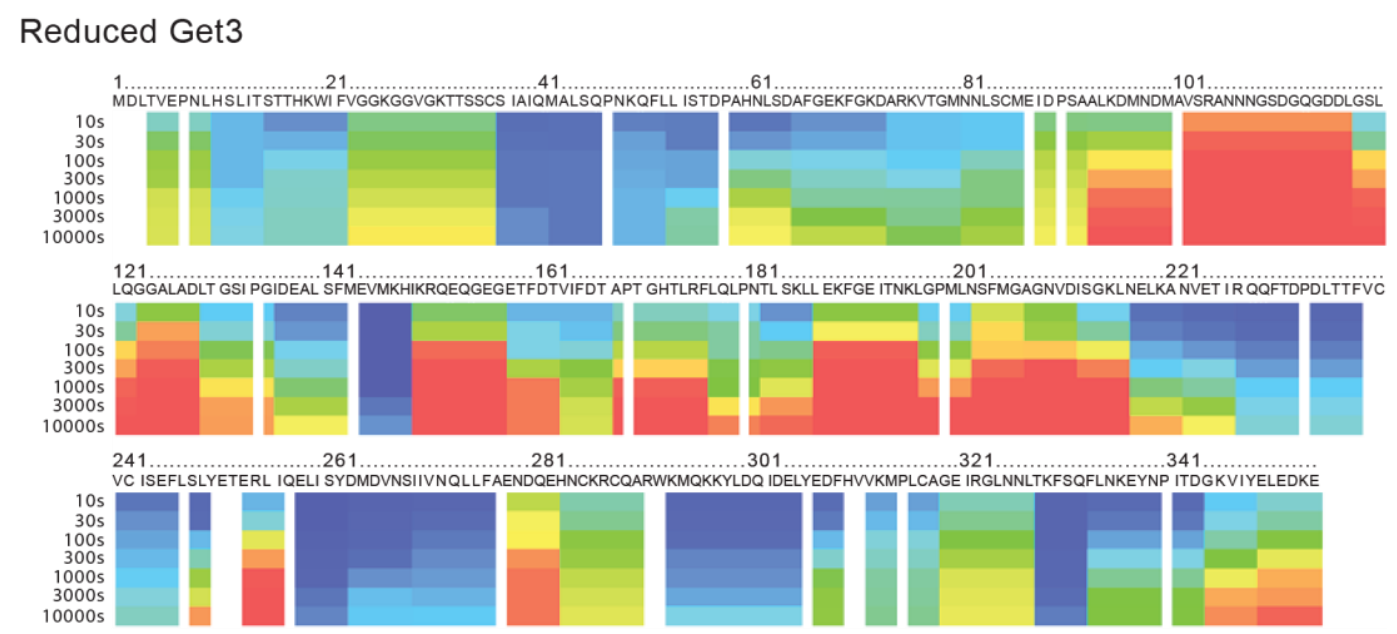

\section{Oxidized Get3}
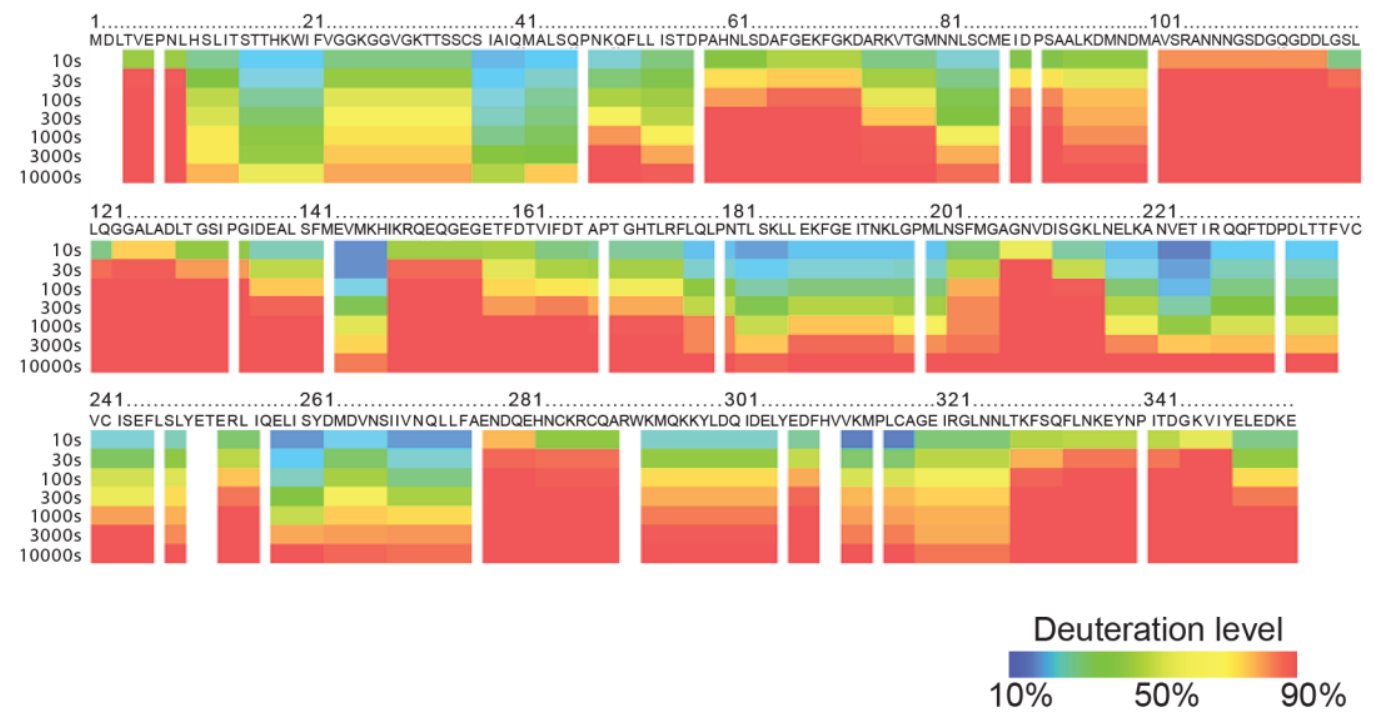

Figure 3.1. 19: Differences in deuterium incorporation between Get $3_{r e d}$ and Get $3_{\text {ox }}$ Deuterium incorporation into peptides of $\mathrm{Get} 3_{\text {red }}$ and $\mathrm{Get} 3_{\text {ox }}$ after quenching and pepsin digest in the presence of 100 mMTCEP. The deuteration le vel was calculated as described in the experimental procedures and mapped on the yeast Get3 protein sequence. Data was provided by Li S (University of California).

differences in individual Get3 peptides over time (Zhang et al 2010).

Analysis of the mass differences for all peptides allowed us to map the degree of deuterium incorporation over time on the Get3 sequence (Figure 3.1.19, reduced and oxidized Get3). Buried, folded or otherwise slowly exchanging regions within Get3 are labeled in blue, whereas less folded, more solvent exposed and more rapidly exchanging regions are 


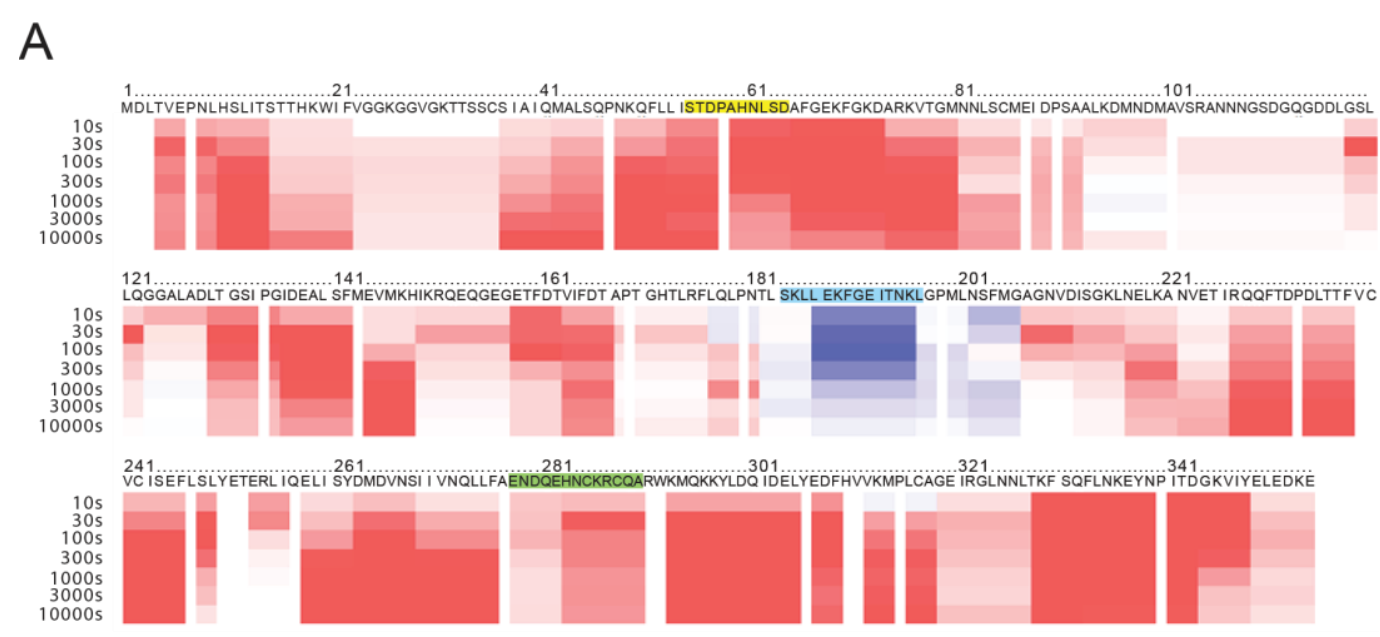

Deuteration level [Get3 ${ }_{\text {ox }}-$ Get3 $\left._{\text {red }}\right]$

B

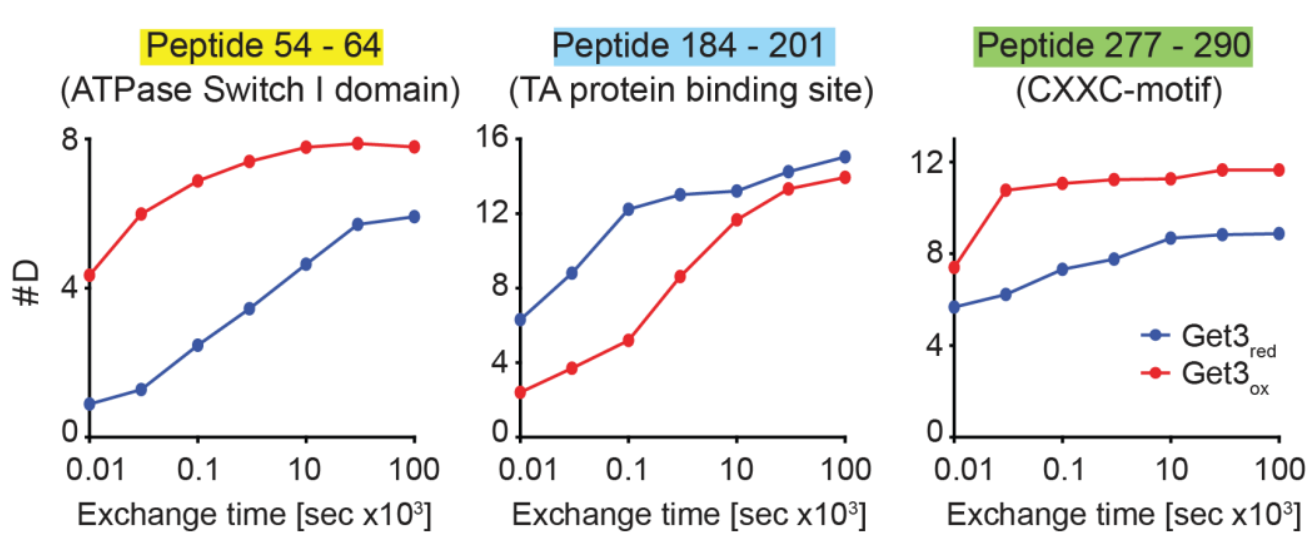

Figure 3.1. 20: Get3 undergoes massive conformational rearrangements upon oxidation

(A) Incorporation of deuterium after select times of hydrogen-deuterium (H/D) exchange into Get $3_{\text {ox }}$ versus Get $3_{\text {red. }}$. Quenching and pepsin digest was performed in the presence of $100 \mathrm{mM}$ TCEP. The deuteration level was calculated as described in the experimental procedures. (B) Direct comparison of the deuterium incorporation over time into select Get3 peptides (indicated as yellow, blue and green shading in A and B) prepared from either Get $3_{\text {red }}$ (blue trace) or Get3 $3_{\text {ox }}$ (red trace). Data was provided by Li S (University of California) and analyzed by Voth W.

shaded in red. Reduced Get3 appears to be significantly more protected against amide proton exchange, indicated by the substantial amount of blue to yellow shaded regions. In contrast, Get3ox is dominated by yellow to red shaded regions, indicating a fast incorporation of deuterium presumably due to more unfolded and/or solvent exposed regions (Figure 3.1.19, reduced and oxidized Get3). Subtraction of deuterium incorporation rates for Get3red 
from Get3ox peptides revealed astounding differences between the two Get3 forms (Voth et al 2014). As indicated by the predominant red shading in Figure 3.1.20, almost every region in Get3ox showed a faster exchange rate with deuterium than the corresponding region in Get3red. These findings are in fully agreement with my previous CD data and indicate oxidation-induced partial unfolding of Get3's structure and/or increase in dynamic properties of the protein. Get3's activation is very similar to other recently identified stress-specific chaperones, like Hsp33 and HdeA, whose activation seems to be triggered by significant protein unfolding (Reichmann et al 2012, Tapley et al 2009).

Next, we projected the differences in deuterium incorporation between Get3ox and Get3red after 100 s of H/D exchange onto Get3's known crystal structure ( $\mathrm{Hu}$ et al 2009) to further visualize the massive structural rearrangements (Figure 3.1.21). As shown in Figure 3.1.21, most of the very crucial changes upon oxidation occur in Get3's ATPase subdomain, including the two conserved cysteine pairs (Cys 242/244), as well as in the Switch I domain, a major component of the ATPase site (Figure 1.6), whose previous mutation led to drastically reduced ATPase activity in Get3 (Powis et al 2013b). These results suggest that the formation of intramolecular disulfide bonds (shown in Figure 3.1.7) leads to a substantial unfolding of Get3's ATPase subdomain. These results are consistent with the finding of oxidation-induced loss in Get3's ATPase activity (Figure 3.1.5 B), which could be attributed to the unfolding of the Switch I domain (Figure 3.1.20 and Figure 3.1.21, yellow). To our big surprise, the only region in Get3ox that showed a slower exchange with deuterium than the corresponding region in Get3red involved residues 184-201 of the alpha-helical subdomain in the Get3 homodimer (Figure 3.1.20 and Figure 3.1.21, blue). This region is thought to form a composite hydrophobic binding site for TA-proteins (Mateja et al 2009). Indeed, very recently published crystallization data show the transmembrane domain of a TAprotein occupied in the hydrophobic groove within Get3's helical subdomain (Mateja et al 2015). Overall, we found strong evidence that oxidative activation of Get3 causes major conformational rearrangements, leading to 


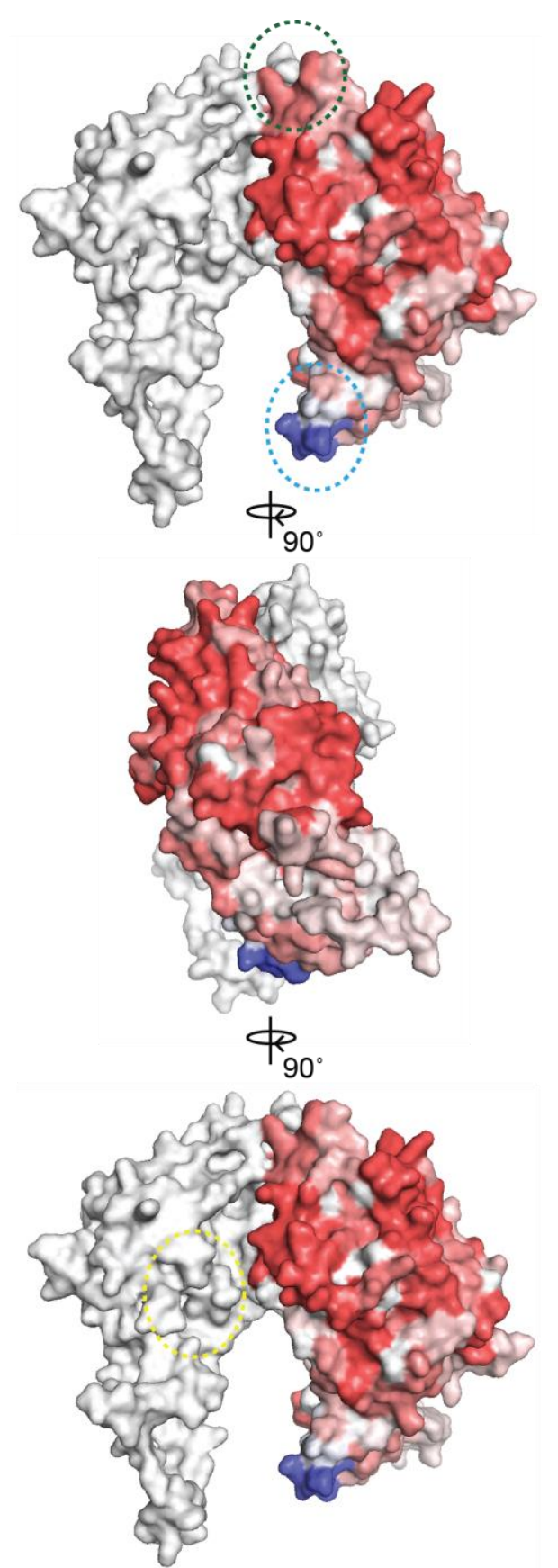

Figure 3.1. 21: Conformational rearrangements mapped on Get3's structure Differences in deuterium incorporation between Get3 $3_{\text {ox }}$ and Get $3_{\text {red }}$ after $100 \mathrm{~s}$ of H/D exchange were mapped on the crystal structure of reduced Get3 (Protein Data Bank ID: 3H84). Locations of peptides shown in Figure 3.1.20 A and B is indicated using colorcoded dotted circles. The molecular surface drawing of the structure was mad by Pymol (The PyMOL Molecular Graphics System, Version 1.5.0.4 Schrödinger, LLC). Data was provided by Li S (University of California) and analyzed by Voth W. 
the exposure of new binding sites for unfolding proteins while masking the binding site for TA-proteins.

\subsubsection{Dissecting the two Get3 functions in vivo}

Our structural analysis revealed that under oxidative stress conditions Get3 potentially unmasks new binding sites for unfolding proteins while burying its binding site for TA-proteins. These results suggested two independent and hence separable protein functions for Get3. Mateja et al. published 2009 a series of Get3 mutant variants, which had apparently lost their TA-protein binding capacity. Briefly, these mutants no longer co-immunoprecipitated with the TA-protein Sec61. Yet, the mutants displayed wild-type-like ATPase activity, indicating that the mutants were likely properly folded. I therefore decided to purify one of the mutant proteins (Get3 1193D), whose I to D mutation was in the center of the proposed TA binding site (Figure 3.1.20 A and $B$ and Figure 3.1.21, blue). We hypothesized, that this mutant protein, which is impaired in TA-protein binding, would still work as a redox-active chaperone and hence should complement get3 phenotypes that require Get3's redox-regulated chaperone function but not the TA-protein targeting function.

I purified the Get3 1193D mutant variant according to the wild-type Get3 purification protocol and subsequently tested its ability to prevent protein aggregation in vitro. As shown in Figure 3.1.22 A and B, analysis of Get3 1193D's chaperone function indeed indicated redox-regulated chaperone activity very similar to wild-type Get3. Increasing concentrations of oxidized Get3 1193D prevented the aggregation of CS, whereas reduced Get3 1193D showed only minor chaperone activity at higher concentrations (Figure 3.1.22 A and B). Next, we tested the Get3 1193D mutant variant for its ability to rescue the oxidative stress phenotypes of the get3 strain (Figure 3.1.1 A). Indeed, our complementation assays showed that the mutant protein was able to fully rescue the growth defect of the deletion strain similar to wild-type Get3 (Figure 3.1.23 A). These results suggested that under oxidative stress conditions, Get3 1193D's chaperone function becomes essential for cell survival. In addition, we also tested the ATPase- 
A

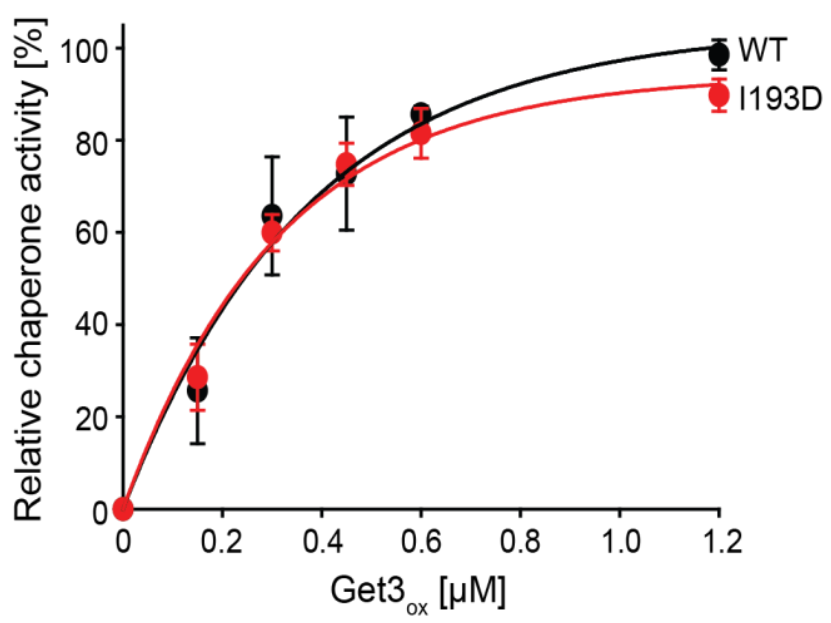

B

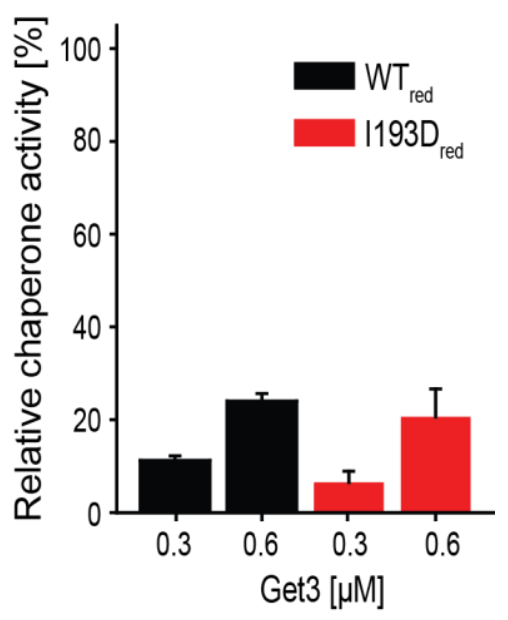

Figure 3.1. 22: Get3's chaperone activity is independent of its TA protein targeting function in vitro

(A) Increasing concentrations of oxidized wild-type Get3 and the TA-binding deficient mutant Get3 I193D and in (B) indicated concentrations of reduced wild-type Get3 and Get3 I193D were tested for their influence on the aggregation behavior of chemically denatured CS upon dilution into buffer (see Figure 3.1.2 for details). Chaperone activity of wild-type Get $3_{\text {ox }}$ was set to $100 \%$ At least 3 replicates were performed and the SE is shown.

deficient Get3 D57E mutant variant and found that it too partially complements the get3 phenotype (Figure 3.1.23 A). These results are consistent with the observation that the chaperone function of Get3 is independent of ATP hydrolysis (Figure 3.1.5 A and B). However, it cannot be excluded that very low residual ATPase activity contributes to the growth rescue under oxidative stress conditions. To further segregate whether Get3's in vivo chaperone function or any residual activity in TA-protein targeting contributes to the growth rescue under stress conditions, we tested the mutant proteins for a potential dominant negative effect in a strain background that not only lacks Get3 but also the Get1/Get2 receptor complex. It has been previously shown that overexpressing of wild-type Get3 is toxic in this strain background (Figure 3.1.23 B), presumably because Get3-bound TA-proteins can no longer be delivered to the ERmembrane (Schuldiner et al 2008). In agreement with our hypothesis that the Get3 1193D mutant variant is no longer capable of binding TA-proteins, 
A

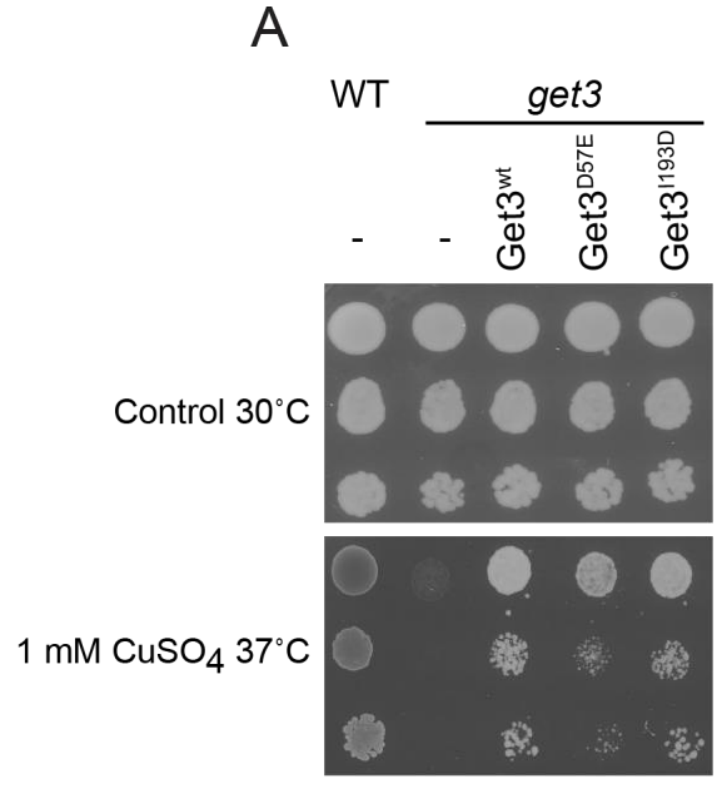

B
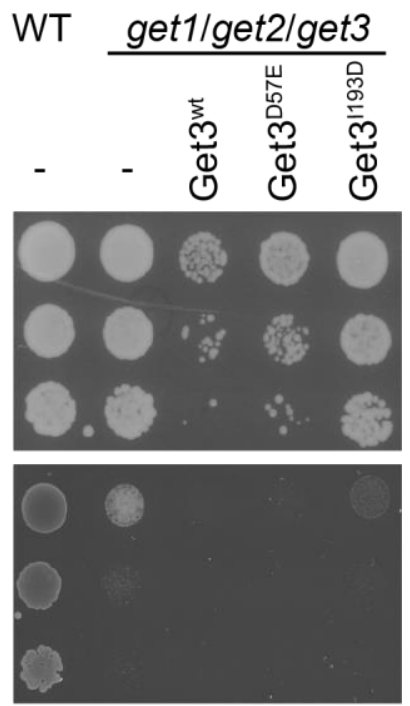

C

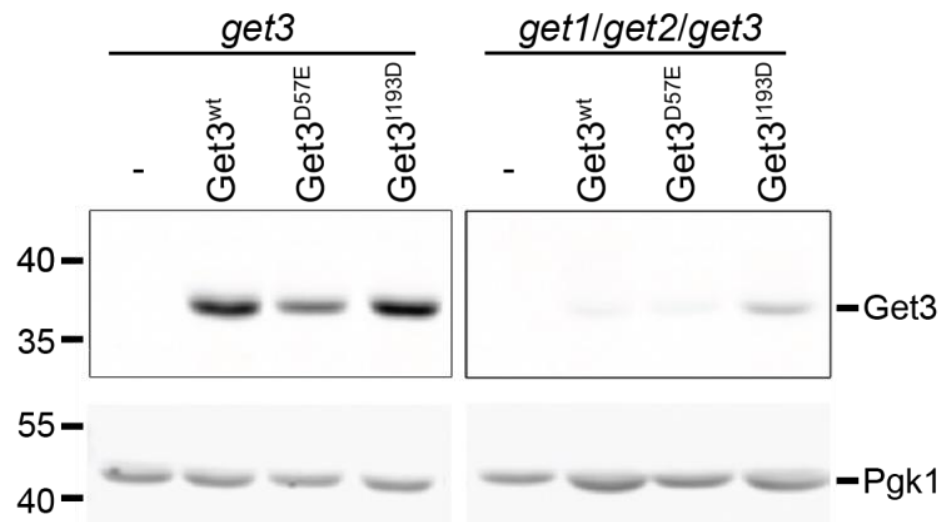

Figure 3.1. 23: Get3 rescues yeast cells under oxidative stress condition independent of its TA-protein targeting

(A) get3 cells and (B) get1/get2/get3 cells were transformed with an empty construct or with constructs containing the coding sequence of wild-type or mutant variants of Get3, respectively. Serial dilutions were then spotted on control plates at $30^{\circ} \mathrm{C}$ or plates containing $1 \mathrm{mM}$ of $\mathrm{CuSO}_{4}$ at $37^{\circ} \mathrm{C}$. Wild-type (BY4741) cells served as control. (C) The same cells were grown over night, lysed by alkaline lysis and analyzed by immunoblot using a Get3 specific serum. Pgk1 was used as loading control. Survival assays and immunoblotting was carried out by Vilardi F (Schwappach Laboratory, Universitätsmedizin Göttingen).

we did not observe any dominant negative effect when we expressed the Get3 1193D mutant protein in these strain (Figure 3.1.23 B). In contrast, expression of the ATPase deficient Get3 D57E mutant showed the expected 
toxic effect, suggesting that it is still able to bind and sequester TA proteins. Surprisingly, however, we did not find any rescue effect upon copper stress conditions in the get1/get2/get3 strain background upon expression of the Get3 1193D mutant protein (Figure 3.1.23 B and C). These results suggest a possible involvement of the GET receptor in regulating the chaperone activity of Get3.

To further strengthen our conclusions that the Get3 1193D mutant variant is no longer involved in TA-protein shuttling, we directly tested it in a wellestablished TA-targeting assay (Jonikas et al 2009). In this assay, we utilized the GFP-tagged TA-protein Sed5, whose insertion into the ER membrane is dependent on the GET pathway, and hence is no longer properly targeted in a get3 deletion strain. We used this strain and coexpressed either an empty plasmid control (mock) or plasmids containing wild-type Get3, the ATPase-deficient Get3 D57E variant or the Get3 1193D mutant (Figure 3.1.24 A). We found that expression of wild-type Get3 properly targeted Sed5 to the ER, allowing subsequent trafficking to the Golgi, as indicated by the presence of multiple puncta (Figure 3.1.24 A, Get3 $\left.{ }^{w t}\right)$. In contrast, get3 cells expressing either of the two mutants showed a much higher cytosolic background with only a few detectable puncta. Location of Sed5 in these cells appeared much more similar to its location in get3 cells expressing the empty control plasmid (Figure 3.1.24 A, compare Mock, Get3 ${ }^{D 57 E}$ and Get3 $\left.{ }^{1193 D}\right)$. Our results are consistent with other reports, in which GFP-Sed5 was mistargeted and moved to

deposition sites for aggregated proteins (Battle et al 2010, Kohl et al 2011, Vilardi et al 2014). A final quantitative analysis of our fluorescence microscopy data confirmed that GFP-Sed5 mistargeting observed in cells expressing either Get3 D57E or 1193D was indeed comparable to the strain lacking Get3. Correctly targeted GFP-Sed5 showed a lower average fluorescence intensity due to the low cytosolic background and had a characteristic shoulder reflecting a higher abundance of very bright pixels found in punctate Golgi structures (Jonikas et al 2009, Vilardi et al 2014) (Figure 3.1.24 B, Get3wt). Mistargeted GFP-Sed5 on the other hand is 


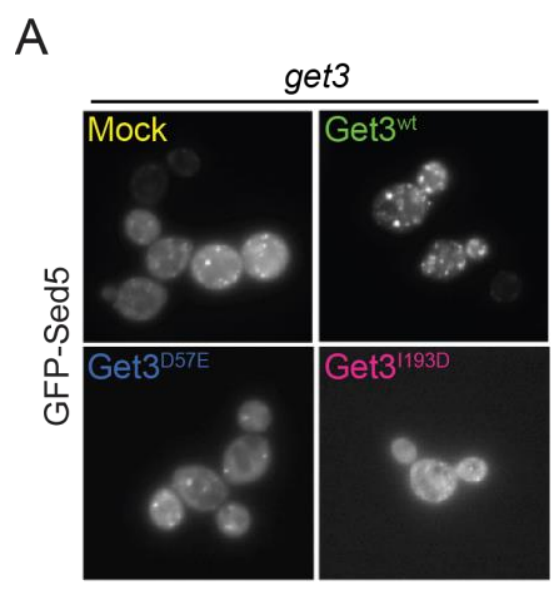

B

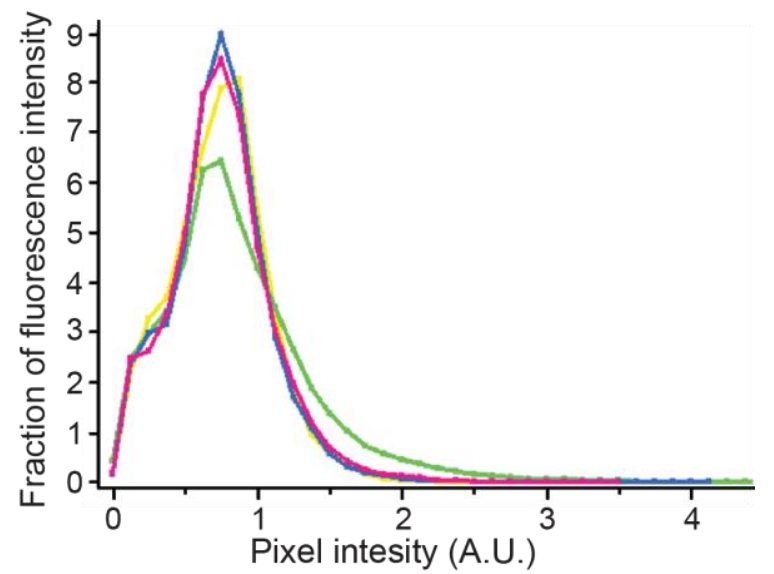

Figure 3.1. 24: Get3 I193D is deficient in TA-protein targeting in vivo

(A) get 3 cells expressing GFP-tagged Sed5 were transformed with an empty construct (Mock = yellow) or with constructs containing the coding sequence of wild -type Get3 (green) or the Get3 D57E (blue) and Get3 I193D (purple) mutant variants. Subcellular localization of GFP-Sed5 was recorded by fluorescence microscopy. (B) At least 40 cells per strain were analyzed to determine the distribution of fluorescence across bins of different pixel fluorescence intensity. Traces are color coded as indicated in A. Imaging and analysis were carried out by Vilardi F (Schwappach Laboratory, Universitätsmedizin Göttingen).

reflected by higher average fluorescence intensity and the absence of bright pixels (Figure 3.1.24 B). Our results are fully consistent with the observation that Get3 protein with an altered hydrophobic groove is incapable of TAprotein targeting or sequestration (Mateja et al 2009). In summary, we present strong evidence that TA-protein targeting activity of get3 can be segregated from its redox-dependent chaperone activity, which appears to be responsible for yeast cell survival under copper-induced oxidative stress conditions.

\subsubsection{Get3 colocalizes with unfolding proteins during oxidative stress in vivo}

In an attempt to visualize Get3's redox-dependent chaperone activity in vivo, we conducted colocalization studies using Get3-GFP and Cherry-Sed5. Under non-stress conditions, Get3 is diffusely distributed in the cytosol whereas the TA-protein is localized to its previously observed distinct Golgi 
A

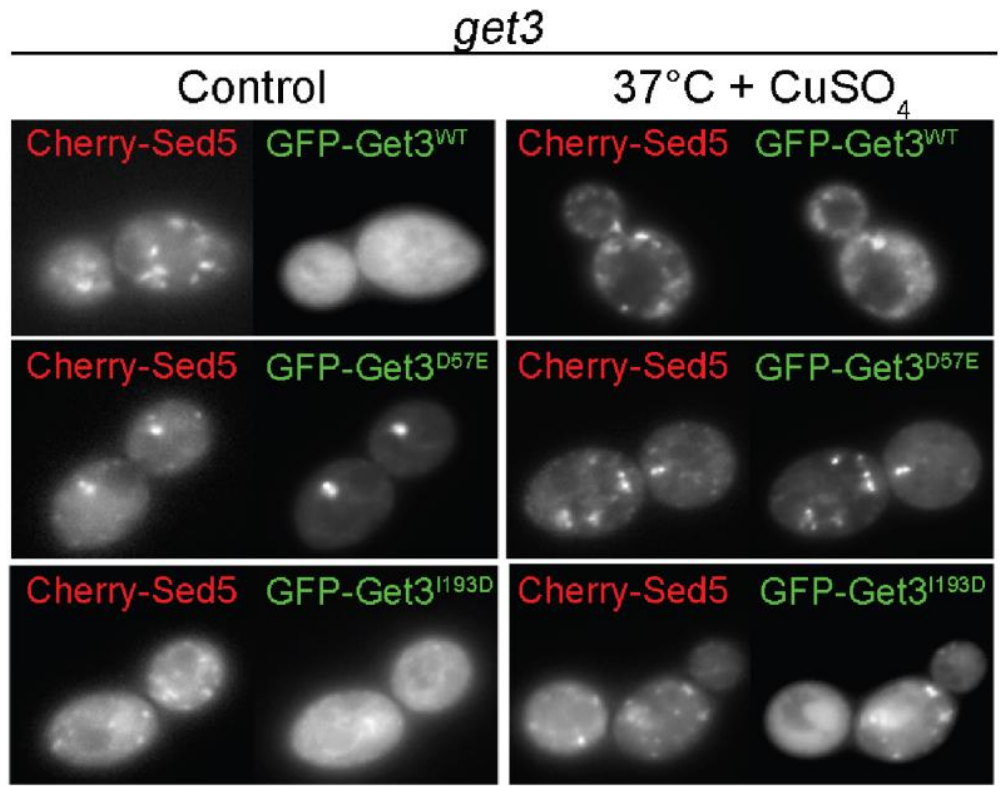

B

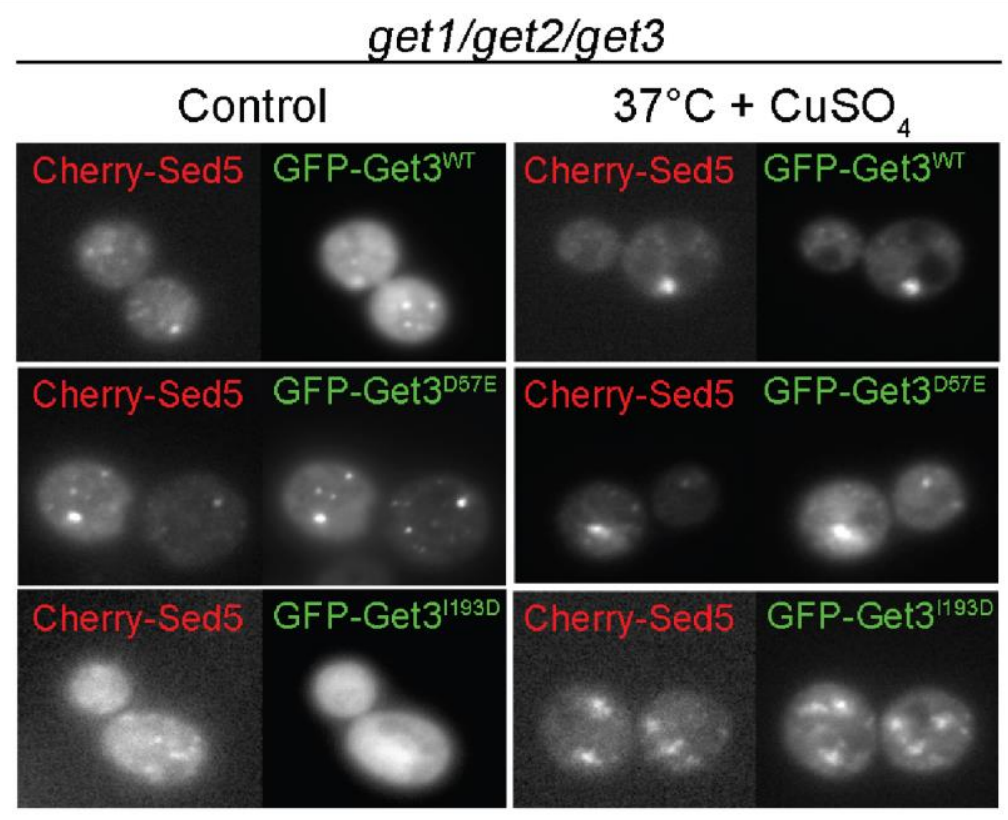

Figure 3.1. 25: Get3 accumulates at deposition sites of mistargeted Sed5 under stress conditions

(A) Yeast cell with get3 or (B) get1/get2/get3 deletions respectively expressing mCherrytagged Sed5 in combination with GFP-tagged Get3 (wild-type or mutant Get3 I193D variants) were grown under control conditions $\left(30^{\circ} \mathrm{C}\right)$ or in presence of $1 \mathrm{mM} \mathrm{CuSO}_{4}$ at $37^{\circ} \mathrm{C}$ for 4 Subcellular distribution of mCherry-Sed5 and GFP-Get3 was recorded by fluorescence microscopy. Imaging was carried out by Vilardi F (Schwappach Laboratory, Universitätsmedizin Göttingen). 
foci (Figure 3.1.25 A, control panel). However, upon exposure of yeast cells to copper stress, Get3-GFP moves to foci which only partially overlap with foci formed by Cherry-Sed5 (Figure 3.1.25 A, right panel). These Get3-GFP foci are reminiscent of previously observed stress-induced co-deposition sites of Get3, aggregated proteins and other chaperones (Powis et al 2013b). A similar redistribution upon exposure to oxidative stress was found for the TA-binding deficient Get3 1193D mutant. This very intriguing finding supports the hypothesis that the recruitment of Get3 to stress foci occurs through oxidative activation of its ATP-independent chaperone function and not through TA-protein binding.

Next, we analyzed the Get3-GFP localization in the receptor-deficient get1/get2/get3 deletion strain to further confirm our conclusions. In this strain background, TA-proteins presumably can no longer be released from Get3 and inserted into the membrane. Indeed, when we imaged wild-type Get3 in this strain background, it appeared to not be freely diffusible but colocalized with Cherry-Sed5 into distinct foci even under non-stress conditions (Figure 3.1.25 B, control panel). These findings are consistent with the previously shown dominant negative effect of wild-type Get3 expression in a strain that lacks the receptor complex (Schuldiner et al 2008) (Figure 3.1.23 B). In agreement with our observation that the Get3 I193D mutant variant no longer binds/inserts TA-proteins, we did not observe a major colocalization of Get3 1193D-GFP with Cherry-Sed5 in the strain lacking the ER receptor proteins (Figure 3.1.25 B, control panel). However, once we challenged the cells with copper stress, Get3 1193D-GFP relocalized into distinct stress foci. Notably, the colocalization of the Get3 D57E mutant variant with Cherry-Sed5 into foci under non-stress conditions was not affected by the absence of the GET receptor nor by the presence of copper or a combination of both (Figure 3.1.25 A and B, compare both panels). As suggested by Powis et al., the reduced ability of Get3 D57E to hydrolyze ATP might directly lead to the deposition of Get3 into stress foci or even trap it there (Powis et al 2013b). Altogether, our in vivo results demonstrate that Get3 exhibits a TA-binding-independent secondary function when challenged by oxidative stress conditions. Hence, 
we can conclude that Get3 plays an important role in protecting yeast cells during protein unfolding and ATP-depletion stress conditions by turning into a potent general chaperone as summarized in a model of Get3's dual function in Figure 4.2, which is based on our in vivo and in vitro results. 


\subsection{The ATP-independent chaperone Get3 protects soluble protein under oxidative stress condition in yeast}

In previous studies we established yeast Get3 as a novel ATP-independent chaperone, whose "holdase" function is rapidly activated under oxidative stress conditions. This stress-specific activation allows oxidized Get3 to protect cells against stress-induced protein aggregation. We found that oxidized Get3 binds both chemically and thermally unfolding substrates and keeps them in solution (Voth et al 2014). This raised the question, however, as to how Get3's substrates are processed upon return to non-stress conditions. We knew from studies with the bacterial Get3 analogue heatshock protein 33 (Hsp33), that this chaperone holdase transfers its substrates to the folding system composed of DnaK/DnaJ/GrpE for refolding once non-stress conditions are restored (Hoffmann et al 2004, Winter et al 2005). We did observe that Get3 reversibly moves to stress foci, and colocalizes with other chaperones, e.g. members of the DnaK family in oxidatively stressed cells, suggesting that Get3 too could interact with other foldases to refold its bound clients (Powis et al 2013b, Voth et al 2014).

\subsubsection{Oxidized Get3 maintains its substrate in a folding competent conformation under oxidative-stress conditions}

First, I investigated the fate of Get3ox-bound substrate upon return to nonstress condition. We had previously established that re-reduction of Get3ox with DTT alone is not sufficient to inactivate its chaperone function. Instead, inactivation of Get3ox required a combination of ATP, DTT and $\mathrm{Zn}^{2+}$. This reversed the massive structural rearrangements in oxidized Get3 and returned the protein to an ATPase-active TA-binding protein (Figure 3.1.1012). To test whether inactivation of Get3ox is sufficient to release the bound

Some results in the chapter 3.2 were obtained in close collaboration with scientists participating in the study: Gates S (Southworth Laboratory, University of Michigan) took all of the micrographs, picked all particles and generated all class averages shown in Figure 3.2.8 and provide the images to me. 


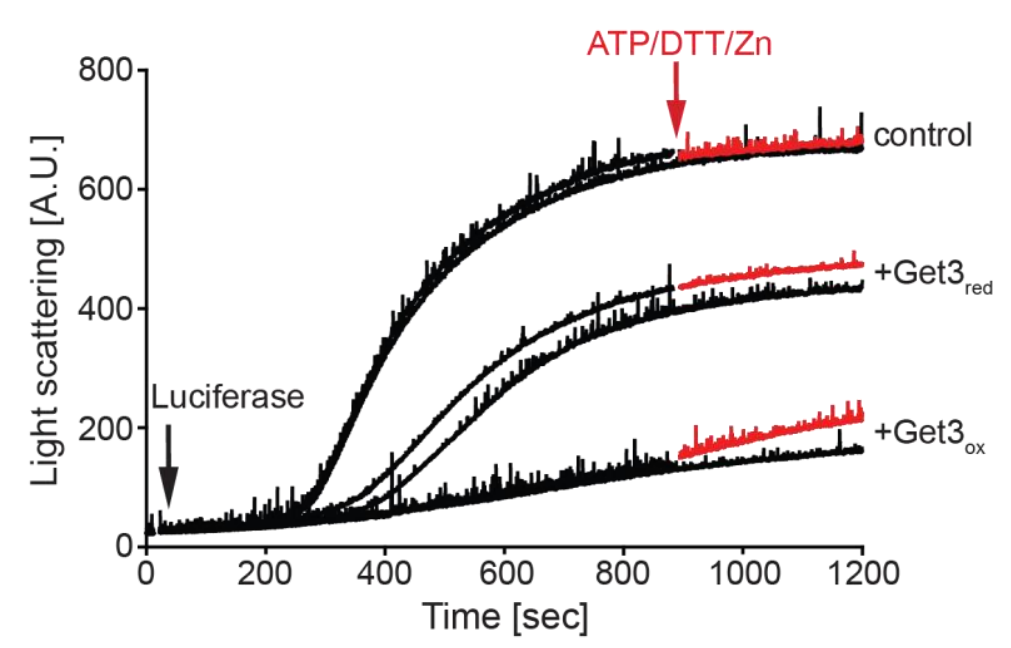

Figure 3.2. 1: Reduction of chaperone active Get ${ }_{\mathrm{ox}}$ in complex with substrate is not sufficient to cause substrate release

Influence of Get3 $3_{\text {red }}$ or Get $3_{\text {ox }}$ on the light scattering of $0.1 \mu \mathrm{M}$ thermally unfolding luciferase at $43^{\circ} \mathrm{C}$ (Get3:luciferase ratio 1:1) upon dilution into buffer was monitored for $1200 \mathrm{sec}$ (black traces). Light scattering of aggregating luciferase measured in the absence of added chaperones is shown (control). At the time point indicated by the red

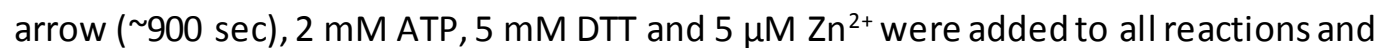
the incubation was continued for $1200 \mathrm{sec}$ at $43^{\circ} \mathrm{C}$ (red traces).

client proteins, I incubated Get3ox and thermally unfolding luciferase at $43^{\circ} \mathrm{C}$ using a 1:1 molar ratio of Get3ox to luciferase. This ratio is sufficient to almost completely prevent the aggregation of luciferase (Figure 3.2.1). We now reasoned that any substrate release from Get3ox during the incubation at $43^{\circ} \mathrm{C}$ would cause luciferase to aggregate further, thereby increasing the light scattering signal. I therefore formed the complex for $900 \mathrm{sec}$ at $43^{\circ} \mathrm{C}$,

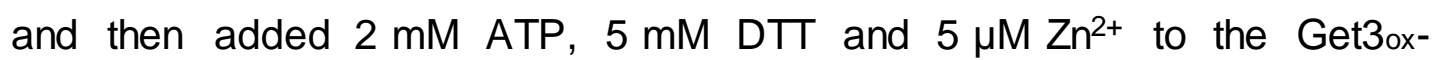
luciferase complex to trigger the inactivation of Get3. Subsequent light scattering measurements revealed only a slight increase in the signal, suggesting the release of only small amounts of luciferase (Figure 3.2.1 A). Addition of ATP, DTT and $\mathrm{Zn}^{2+}$ to thermally aggregated luciferase alone or in the presence of Get3red had no effect. These results suggested that inactivation of oxidized Get3's chaperone function alone is not sufficient to completely release chaperone bound substrates.

To investigate whether Get3 has the ability to release its bound client proteins in a folding competent state upon return to non-stress conditions, 


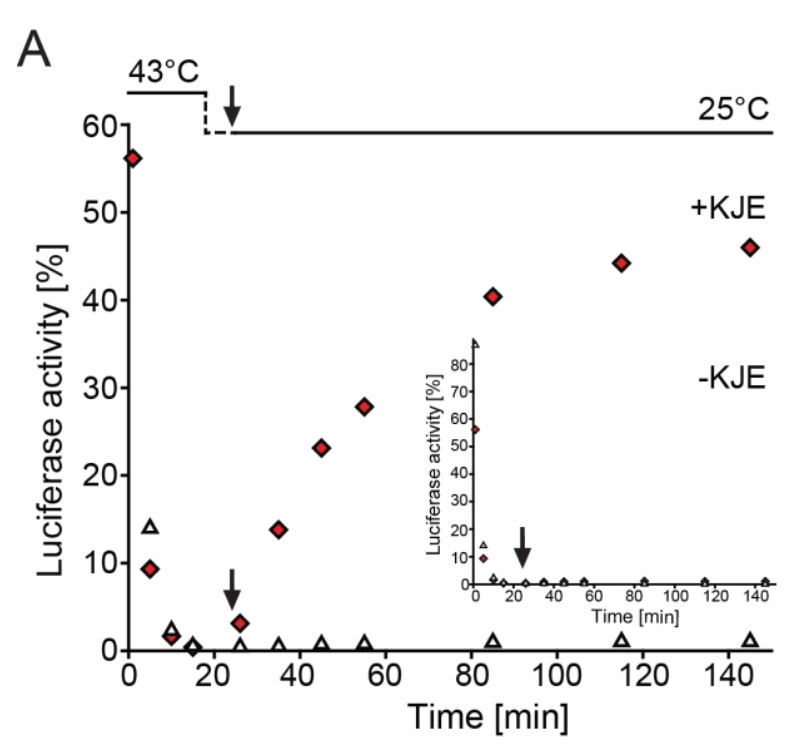

B

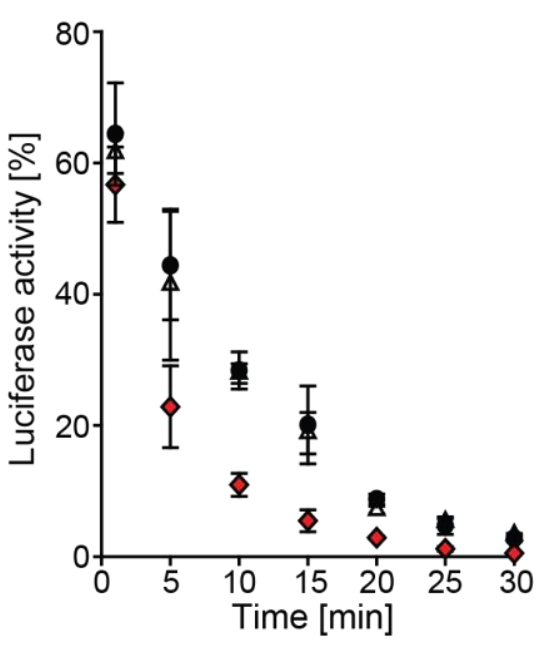

Figure 3.2. 2: Substrate binding and transfer ability of Get3 is redox-dependent (A) Luciferase $(0.1 \mu \mathrm{M}$ ) alone (white triangle) or in the presence of a stoichiometric molar ratio of Get $3_{\text {ox }}$ (red diamonds) was incubatedfor $15 \mathrm{~min}$ at $43^{\circ} \mathrm{C}$. The samples were then cooled to $25^{\circ} \mathrm{C}$ and after 10 min supplemented with $5 \mathrm{mM}$ DTT, $2 \mathrm{mM} \mathrm{MgATP}$ and $0.1 \mathrm{mg} / \mathrm{ml}$ BSA (black arrow). Where indicated, a 20:4:20:1 ratio of DnaK:DnaJ:GrpE (KJE) system to luciferase was added. Luciferase inactivated alone or in the presence of Get $3_{\text {ox }}$ supplemented with DTT/ATP in the absence of KJE were monitored (inset, white circles). At the indicated time points aliquots were taken and luciferase activity was assayed. (B) $0.1 \mu \mathrm{M}$ of Luciferase alone (white triangle) or in the presence of stoichiometric molar ratios of either Get $3_{\text {ox }}$ (red diamonds) or Get $3_{\text {red }}$ (black circles) was incubated for 30 min at $39^{\circ} \mathrm{C}$ to slow down the inactivation. At the indicated time points aliquots were taken and luciferase activity was assayed. Luciferase prepared at room temperature prior to the inactivation was tested and set to $100 \%$ activity. However, since luciferase loses almost $50 \%$ of its activity upon incubation at room temperature, it is not possible to fully recover (100\%) its activity. At least 3 replicates were performed and the SE is shown.

we monitored the effect of Get3ox on the activity of luciferase exposed to thermal unfolding conditions. We therefore incubated luciferase alone or in the presence of chaperone-active Get3ox at a $1: 1$ molar ratio at $43^{\circ} \mathrm{C}$ and assayed luciferase activity at defined time points. Over the time course of the incubation, luciferase activity decreased drastically. After $15 \mathrm{~min}$ of incubation, the activity was below $1 \%$. Surprisingly, the presence of Get3 ox even further accelerated the inactivation of luciferase (Figure 3.2.2 A, compare white triangle and red diamonds), suggesting that Get3ox binding directly enhanced the unfolding of luciferase. Subsequently, I shifted the temperature to $25^{\circ} \mathrm{C}$ and monitored luciferase activity upon addition of 
DTT/ATP, expecting that any slow release of folding competent luciferase should lead to its refolding and hence to the recovery of luciferase activity. However, no significant luciferase reactivation was observed (Figure 3.2.2 A inset, red diamonds), indicating that Get3ox is not able to release its substrates in a refolding-competent conformation. This result is in agreement with other ATP-independent stress-specific chaperones such as bacterial Hsp33 or mitochondrial peroxiredoxin (Prx) from Leishmania infantum, which are unable to refold their client proteins and instead transfer them to ATP-dependent folding systems, such as the heat-shock protein 40 (Hsp40) and Hsp70 system (Hoffmann et al 2004, Teixeira et al 2015). To test whether upon reduction, Get3ox also transfers its clients to the bacterial DnaK/DnaJ/GrpE (KJE) system, which is homologous to the eukaryotic Hsp40/70 systems (Daugaard et al 2007), we added the bacterial KJE system immediately upon the shift to $25^{\circ} \mathrm{C}$ in the presence of DTT/ATP (ATP is also needed for the DnaK-function). We found that addition of the KJE system to the Get3ox:luciferase complex in the presence of DTT/ATP resulted in a substantial increase in luciferase activity while addition of KJE to luciferase inactivated in the absence of Get3ox showed no significant luciferase reactivation (Figure 3.2.2 A, compare red diamonds and white triangles).

To analyze the apparently stimulating influence of Get3ox on the unfolding of luciferase in more detail, we incubated luciferase at $39^{\circ} \mathrm{C}$, which significantly slowed down the inactivation process (Figure 3.2.2 B). As expected, inactive Get3red showed no detectable influence on the inactivation kinetics of luciferase (Figure 3.2.1 B). In contrast, however, the presence of Get3ox substantially accelerated the inactivation of luciferase compared to the inactivation kinetics of luciferase incubated in the absence of additional proteins (Figure 3.2.2 B). These results suggest a very tight binding between chaperone active Get3 and early unfolding intermediates of luciferase, which shifts the equilibrium between folded and unfolding substrate towards the unfolded state, and hence increases the rate of unfolding. Similar results have previously been observed with other chaperones, such as GroEL (Walter et al 1996). 
A

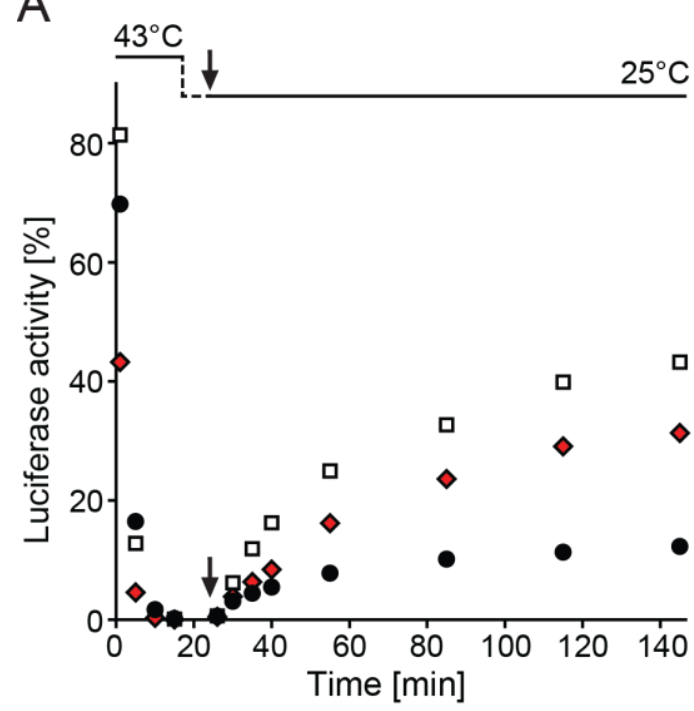

B

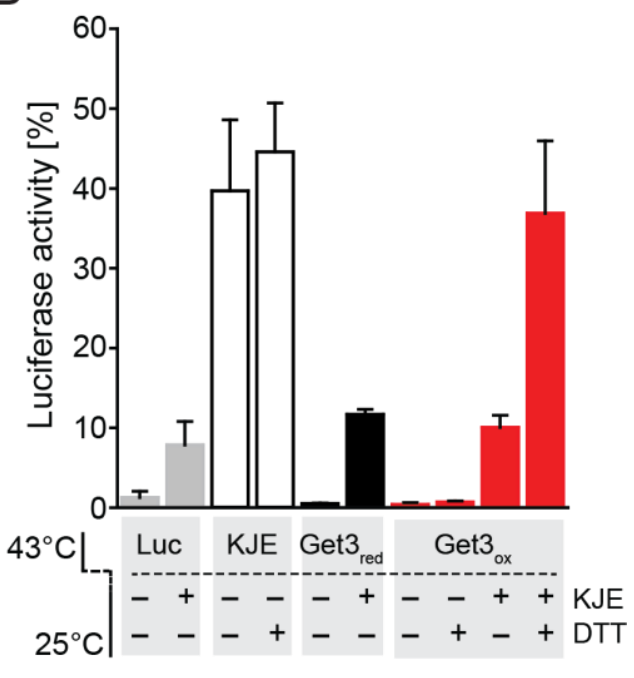

Figure 3.2. 3: Get $3_{\mathrm{ox}}$ maintains luciferase in a refolding competent state

(A) Luciferase $(0.1 \mu \mathrm{M})$ was incubated in the presence of stoichiometric molar ratios of either Get $3_{\text {ox }}$ (red diamonds) or Get $3_{\text {red }}$ in the presence of $2 \mathrm{mM} \mathrm{DTT}$ (black circles) for $15 \mathrm{~min}$ at $43^{\circ} \mathrm{C}$. All samples were cooled to $25^{\circ} \mathrm{C}$ and after 10 min supplemented with $2 \mathrm{mM} \mathrm{MgATP}, 0.1 \mathrm{mg} / \mathrm{ml} \mathrm{BSA}$ and up to $5 \mathrm{mM}$ DTT (black arrow) to restore non-stress conditions and the KJE system (to Get3 ox and Get3 $3_{\text {red }}$ samples) as described in Figure 3.2.2 to refold luciferase. At indicated time points aliquots were taken and luciferase activity was tested. Luciferase inactivated in the presence of the KJE system (white squares) served as a control for maximal refolding of luciferase. (B) $0.1 \mu \mathrm{M}$ luciferase was inactivated in the presence of $2 \mathrm{mM}$ DTT and in the absence of any chaperone system (Luc, light gray bars), in the presence of a 20:4:20:1 ratio of DnaK:DnaJ:GrpE (KJE) system to luciferase (white bars), or in the presence of either Get3 $3_{\text {red }}$ and 2 mM DTT (black bars) or Get $3_{\text {ox }}$ (red bars) at $43^{\circ} \mathrm{C}$ for 15 min. Subsequently, the temperature was shifted to $25^{\circ} \mathrm{C}$ for $10 \mathrm{~min}$, and $2 \mathrm{mM} \mathrm{MgATP}$ and $0.1 \mathrm{mg} / \mathrm{ml} \mathrm{BSA}$ were added to all samples. Additionally, all samples except those that already contained the KJE system were supplemented with the KJE system at previously described ratios or with up to $5 \mathrm{mM} \mathrm{DTT}$, or with a combination of both. After $120 \mathrm{~min}$ of incubation at $25^{\circ} \mathrm{C}$ luciferase activity was assayed. Luciferase prepared at room temperature prior to the inactivation was tested and set to $100 \%$ activity. 3-6 replicates were performed and the $\mathrm{SE}$ is shown.

In the next set of experiments, I directly compared the substrate transfer ability of Get3red and Get3ox. Therefore, luciferase was inactivated in the presence of Get3red or Get3ox at $43^{\circ} \mathrm{C}$. As before, we shifted the temperature to $25^{\circ} \mathrm{C}$ and added the bacterial KJE system and ATP. It is of note that the inactivation with Get3red was carried out in the presence of $2 \mathrm{mM}$ DTT to ensure reducing conditions, making the additional supplementation with DTT during the non-stress incubation obsolete. Importantly, presence of 
DTT had no significant influence on the kinetics of luciferase inactivation (data not shown). Upon measuring the activity of luciferase upon incubation at non-stress temperatures, we found that presence of Get3red had no influence on luciferase refolding (Figure 3.2.3 $\mathrm{A}$ and $\mathrm{B}$ ). These results confirmed our previous conclusion that Get3red is unable to form stable complexes with unfolded clients and hence is not able to prevent their unfolding/aggregation. In contrast, Get3ox tightly binds to unfolding clients, maintains them in a folding-competent conformation and upon return to nonstress conditions, transfers them to ATP-dependent chaperone foldases for refolding (Figure 3.2.3 A and B). Interestingly, addition of KJE to Get3ox in the absence of DTT showed only a minor luciferase reactivation (Figure 3.2.3 B), indicating that the release of the tightly bound substrate is redox dependent.

To confirm that Get3ox transfers clients also to its eukaryotic partner proteins, we conducted the same experiments but used yeast Hsp40 (YdJ1) and human Hsp70 (HSPA1A) to support luciferase refolding. Unlike the prokaryotic DnaK/DnaJ/GrpE-system, the eukaryotic Hsp40/70 system does not require a nucleotide exchange factor (i.e., GrpE). Furthermore, the components are interchangeable between species, hence human Hsp70 is compatible with yeast Hsp40 and vice versa without major loss of activity (Lee \& Vierling 2000). First, I tested the refolding capacity of Ydj1 and HSPA1A according to previously performed refolding studies (Haslbeck et al 2005b). Therefore, I inactivated $0.1 \mu \mathrm{M}$ luciferase alone or in the presence of $1 \mu \mathrm{M}$ human Hsp70 and $2 \mu \mathrm{M}$ yeast Ydj1 (1:10:20 ratio) and initiated the refolding of luciferase by shifting the temperature to $25^{\circ} \mathrm{C}$ and the addition of ATP. Luciferase activity measurements revealed that the combination of HSPA1A and Ydj1 reactivated up to $25 \%$ of luciferase within three hours of incubation. In contrast, we observed less than $4 \%$ reactivation when luciferase was inactivated in the absence of any chaperones (Figure 3.2.4 B, compare light gray and white bars). These results were consistent with previous reports that refolding of luciferase in the presence of the eukaryotic Hsp40/70 systems requires extended incubation times of at least three hours (Haslbeck et al 2005b, Lee \& Vierling 2000). Next, I 
A

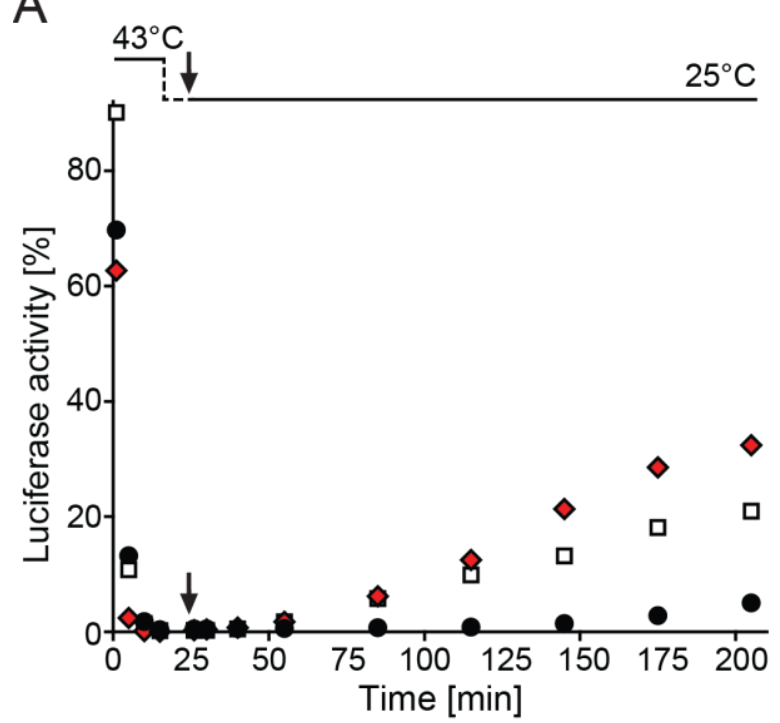

B

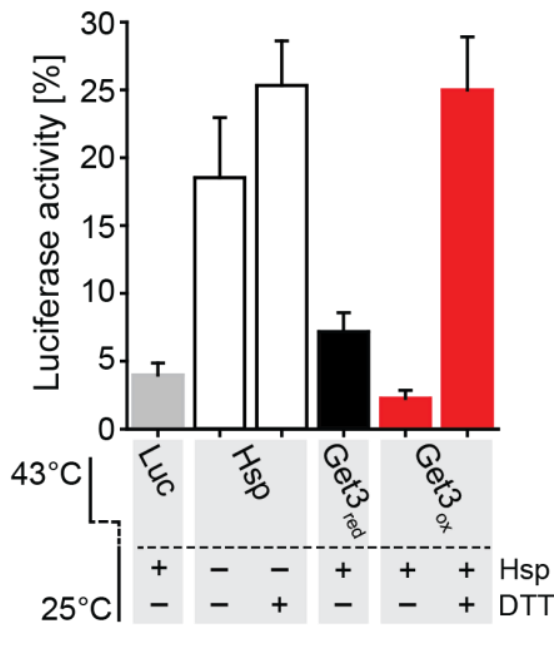

Figure 3.2. 4: Get3's substrate transfer to eukaryotic Hsp40/70 is redox dependent (A) $0.1 \mu \mathrm{M}$ luciferase was inactivated in the presence of stoichiometric molar ratios of either Get $3_{\text {ox }}$ (red diamonds) or Get $3_{\text {red }}$ in the presence of $2 \mathrm{mM}$ DTT (black circles) for $15 \mathrm{~min}$ at $43^{\circ} \mathrm{C}$. After a cool-down to $25^{\circ} \mathrm{C}$ for $10 \mathrm{~min}$ all samples were supplemented with $2 \mathrm{mM} \mathrm{MgATP}, 0.1 \mathrm{mg} / \mathrm{mL} \mathrm{BSA}$ and up to $5 \mathrm{mM}$ DTT (black arrow). In addition, a 20:10:1 ratio of the Ydj1:HSPA1A (Hsp40/70) system to luciferase was added to the Get $_{\text {red }}$ and Get $_{\text {ox }}$ samples. At indicated time points aliquots were collected and luciferase activity was measured. Luciferase inactivated in the presence of the Hsp40/70 system (white squares) served as a control. (B) Luciferase $(0.1 \mu \mathrm{M})$ was inactivated in the absence of any chaperone system (Luc, light gray bars), in the presence of a 20:10:1 ratio of the Ydj1:HSPA1A (Hsp40/70, dark gray bars) system to luciferase, or in the presence of either Get $3_{\text {red }}$ (black bars) or Get $3_{\text {ox }}$ (red bars) at $43^{\circ} \mathrm{C}$ for $15 \mathrm{~min}$. The temperature was shifted to $25^{\circ} \mathrm{C}$ for $10 \mathrm{~min}$, and $2 \mathrm{mM} \mathrm{MgATP}$ and $0.1 \mathrm{mg} / \mathrm{mLBSA}$ were added to all samples. Additionally, all samples except those that already contained the Hsp40/70 system were supplemented with the $\mathrm{Hsp} 40 / 70$ system at previously indicated ratios or with up to $5 \mathrm{mM} \mathrm{DTT}$, or with a combination of both. After $180 \mathrm{~min}$ of incubation at $25^{\circ} \mathrm{C}$ luciferase activity was assayed. Luciferase prepared at room temperature prior to the inactivation was tested and set to $100 \%$ activity. 3-6 replicates were performed and the $\mathrm{SE}$ is shown.

tested Get3red and Get3ox for their substrate transfer ability to HSPA1A/Ydj1. Therefore, I inactivated luciferase in the presence of either Get3red or Get3ox and induced refolding similar to our previous experiments. Addition of the Hsp40/70 system and DTT to pre-formed Get3ox:luciferase complexes resulted in a substantial increase in luciferase reactivation (Figure 3.2.4 A and $\mathrm{B}$, red diamonds and bars). In contrast, luciferase inactivated in the presence of Get3red showed significantly less recovery of $\sim 6 \%$ (Figure 3.2.4 
$A$ and $B$, black circles and bars), which was similar to luciferase incubated alone (Figure 3.2.4 B, white bar). These results are consistent with the refolding assays conducted in the presence of the KJE system, and confirmed that Get3ox in contrast to Get3red maintains its client protein in a folding competent conformation under stress condition and can transfer its clients to ATP-dependent chaperone systems of different species. Furthermore, my results are consistent with the observation that Get3 colocalizes with members of the Hsp70 family under stress conditions (Powis et al 2013b), suggesting that Get3 might also interact with the Hsp70 system in living cells.

\subsubsection{Visualizing the Get $3_{\text {tet }}$ - luciferase complex}

In previous studies, we analyzed the oligomerization process of Get3 upon oxidation and identified a Get3ox tetramer (Get3tet) as the smallest chaperone-active species (Figure 3.1.14-15) (Voth et al 2014). To further characterize and potentially visualize the Get3tet-substrate complexes by EM, I prepared a homogeneous mixture of Get3tet-luciferase complexes, avoiding the presence of any unbound luciferase that might form aggregates. First, I purified Get3tet in large quantities as previously described in chapter 3.8. Then, I tested various molar ratios of Get3 to luciferase for complex formation. Increasing concentrations of Get3 (up to $1.2 \mu \mathrm{M}$ ) were incubated with $0.15 \mu \mathrm{M}$ luciferase in $40 \mathrm{mM}$ HEPES, $50 \mathrm{mM}$ $\mathrm{KCl}, \mathrm{pH} 7.5$ buffer at $20^{\circ} \mathrm{C}$. Then, I gradually increased the temperature up to $43^{\circ} \mathrm{C}$ to induce complex formation. The individual samples were centrifuged at full speed for 30 min to separate the soluble supernatant (i.e., Get3-luciferase complexes) from the insoluble pellet (i.e. luciferase aggregates), and the fractions were analyzed on reducing SDS-PAGE. As shown in Figure 3.2.5 A, only in the presence of the highest Get3tet concentrations, luciferase was kept completely soluble during the thermal incubation. We therefore prepared complexes using a molar ratio of Get3 to luciferase of $8: 1$ and $4: 1$, and analyzed them by analytical size exclusion chromatography (Superdex200). The elution profile for the Get3tet-lucife rase complex (8:1 ratio) revealed a peak at $11.6 \mathrm{ml}$ that was shifted by $0.5 \mathrm{ml}$ 
A

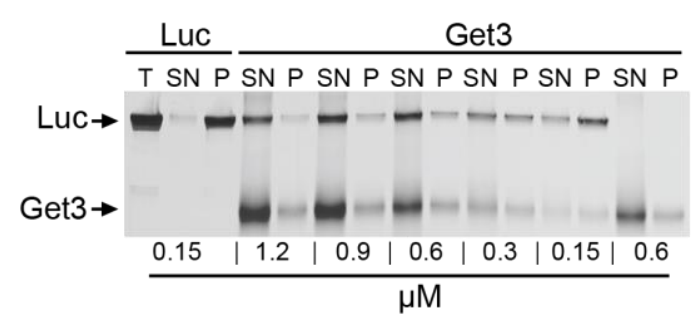

B

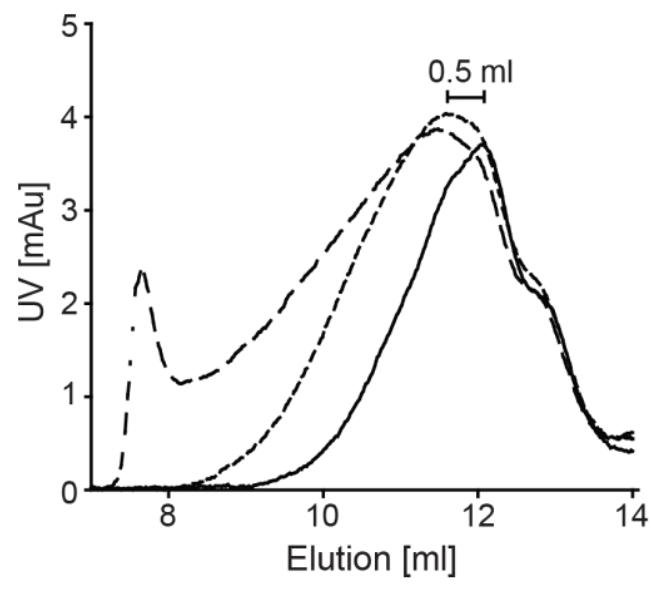

Figure 3.2. 5: The oxidized Get3 ${ }_{\text {tet }}$ forms a stable complex with luciferase

(A) $0.15 \mu \mathrm{M}$ luciferase (Luc) alone or in combination with indicated concentrations of purified Get $_{\text {tet }}$ (Get3) was incubated in $40 \mathrm{mM}$ HEPES, $50 \mathrm{mM} \mathrm{KCl}, \mathrm{pH} 7.5$ buffer at gradually increasing temperatures, rising from $25^{\circ} \mathrm{C}$ up to $43^{\circ} \mathrm{C}$ over the time course of $22 \mathrm{~min}$. Subsequently, the samples were pelleted by centrifugation and the soluble fraction in the supernatant $(S N)$ and the insoluble fraction in the pellet $(P)$ were ana lyzed on reducing SDS-PAGE. Get $3_{\text {tet }}$ incubated al one servedas control. (B) Get $3_{\text {tet }}$ alone (solid line) or Get $3_{\text {tet }}$-luciferase complexes formed with a molar ratio of Get3 to luciferase of 4:1 (long dashed line) and 8:1 (short dashed line) were analyzed on size exclusion chromatography (Superdex 200 10/300 GL column).

compared to the peak of Get3tet alone (Figure 3.2.5 B). This shift in elution corresponds to a size increase of $\sim 45 \mathrm{kDa}$, suggestive of complex formation with luciferase $(\sim 60 \mathrm{kDa})$. Consistent with the supernatant-pellet fractionation result, Get3tet-luciferase complexes that were formed at the lower molecular ratio $(4: 1)$ revealed the additional presence of partially aggregated protein in the void volume of the column ( 8 $\mathrm{ml})$ (Figure 3.2.5 B). It is of note, that the Get3 to luciferase ratio necessary to keep luciferase soluble and prevent aggregation was substantially higher $(8: 1)$ in these experiments as compared to our previous studies shown in Figure 3.2.1. Unlike thermal aggregation assays which are conducted in a cuvette under permanent stirring conditions, the formation of large quantities of complexes in a test tube requires higher concentrations of chaperone to avoid aggregation of luciferase and ensure a homogeneous complex formation (see material and methods part for details (Figure 2.2)). Our results were in agreement with our hypothesis that one Get3 tetramer (molar 
A

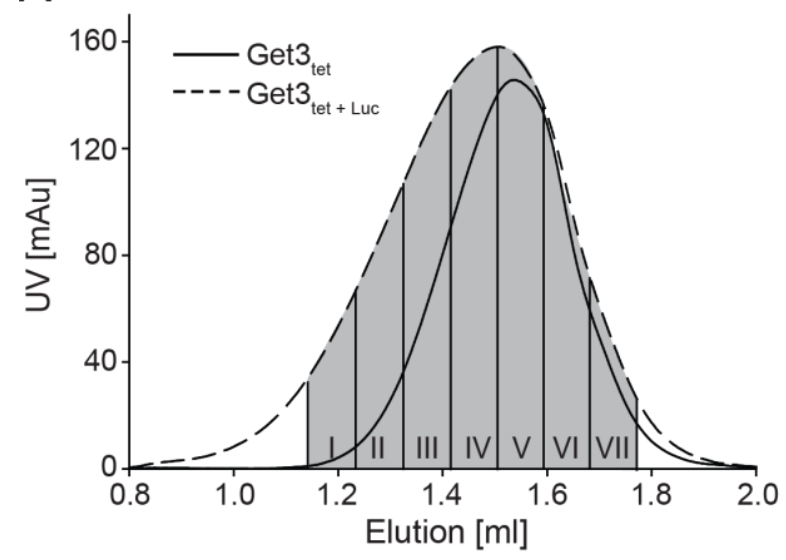

B

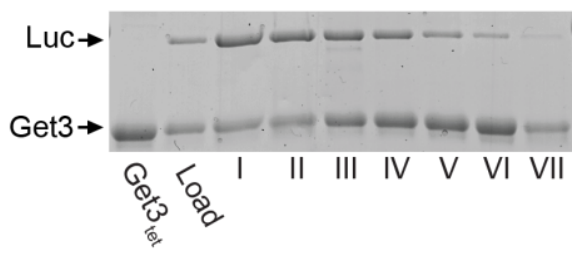

Figure 3.2. 6: Purification of a homogeneous Get $3_{\text {tet }}$-luciferase complex

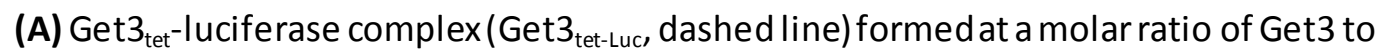
luciferase of 8:1 was purified on a highly resolving size exclusion chromatography (Suprose6pc column). Indicated fractions (gray shaded areas) containing higher oligomers with various molar Get3 to luciferase ratios were collected (fractions I-VII)and (B) subsequently analyzed on reducing SDS-PAGE. Get3 $3_{\text {tet }}$ alone (solid line) served as control. Luciferase (LuC) and Get $3_{\text {tet }}$ (Get3) separated by SDS-Page are indicated. Loading control (Load) of Get $3_{\text {tet }}$-luciferase complex before separation on the gel filtration column is shown.

Get3 to luciferase ratio of 4:1) binds to one luciferase. Alternatively, it is also possible that two tetramers bind and shield one luciferase molecule within their respective clefts. To ensure the homogeneity of the complex, we therefore continued with a complex formed at a molar ratio of Get3ox to luciferase of 8:1. The preformed Get3tet-luciferase complexes were then applied onto a micro-preparative size exclusion column (Suprose6pc) to further purify the complex. As indicated in Figure 3.2.6 A, the complex eluted as a broad single peak at about $1.5 \mathrm{ml}$. The peak was shifted compared to the peak of Get3tet alone, indicating complex formation and confirming our previous test runs (Figure 3.2.5 B). Individual fractions of the peak were then collected and analyzed by SDS-PAGE. We found that the fractions that eluted earlier contained a much higher luciferase to Get3tet ratio than later eluting fractions (Figure 3.2.6 $\mathrm{A}$ and $\mathrm{B}$, indicated fractions within shaded area on gel). These results indicate that the Get3-luciferase complexes likely vary in the molar Get3 to luciferase ratio. To increase the homogeneity of the complexes, I collected fractions II - N and re- 
A

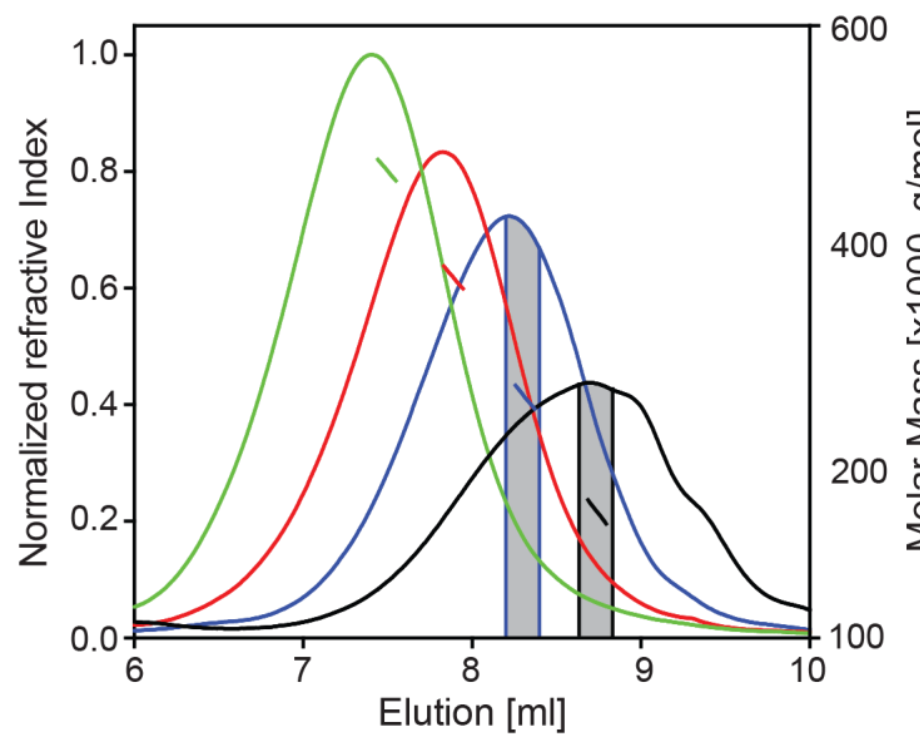

B

\begin{tabular}{c|c} 
& $\mathrm{MW}[\mathrm{kDa}]$ \\
\hline Get3 $_{\text {tet }}$ & 160 \\
\hline II & 580 \\
\hline III & 390 \\
\hline IV & 245
\end{tabular}

C

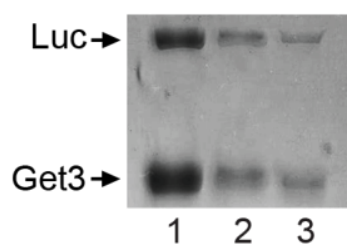

Figure 3.2. 7: The Get $3_{\text {tet }}$-luciferase complex forms oligomers of various sizes

(A) Get $3_{\text {tet }}$ and various Get $3_{\text {tettluc }}$ sub-fractions of the elution shown in Figure 3.2.6 A (II, III and IV) were analyzed by SEC-MALS and (B) calculated to be approximately $160 \mathrm{kDa}$, $580 \mathrm{kDa}, 390 \mathrm{kDa}$ and $245 \mathrm{kDa}$ in size, respectively. This sizes are consistent with a Get $3_{\text {tet }}$ and $\mathrm{Get} 3_{\text {tet }}$-luciferase complexes of various sizes and molar ratios. The areas shaded in light gray were latter analyzed on negative stain electron microscopy (EM). (C) Freshly prepared Get $3_{\text {tet }}$-luciferase complex (1), sub-fractions IV of the elution shown in Figure 3.2.6 A (2) and a sub-fraction of the MALS elution (blue curve, light gray shaded area) shown in A (3) were analyzed on reducing SDS-Page. Molar Get3 to luciferase ratios were calculated using a Get3/luciferase standard of various concentrations and corresponded for (1) to an 8:1, for (2) to an 8:1 and for (3) to an 9:1 ratio, respectively.

chromatographed them using a size exclusion column connected to a static light scattering instrument for molecular weight determination (SEC-MALS). Whereas fractions II and III eluted as broad peaks at about $7.4 \mathrm{ml}(580 \mathrm{kDa})$ and $8 \mathrm{ml}(390 \mathrm{kDa})$, the proteins of fraction IV eluted as a sharp peak at about $8.3 \mathrm{ml}$ which corresponds to a molecular weight of about $245 \mathrm{kDa}$ (Figure 3.2.7 A and B, gray shaded are under blue curve). Since the average molecular weight for Get3tet alone was calculated to be $160 \mathrm{kDa}$ and luciferase has a molecular weight of $60 \mathrm{kDa}$, this result suggests the fraction IV contains complexes between Get3 tetramers and one partially unfolded and potentially elongated luciferase molecule. Subsequent analysis of the peak fractions of this MALS run (Figure 3.2.7 A, shaded area under blue curve) on reducing SDS-PAGE, revealed a Get3 to luciferase ratios of about 
9:1 (Figure 3.2.7 C). This results suggest the presence of a single Get3tetluciferase complex contaminated with empty Get3tet.

We next conducted negative-stain EM on the peak fraction of the SECMALS elution (Figure 3.2.7, shaded area $245 \mathrm{kDa}$ and $160 \mathrm{kDa}$ ) to visualize the architecture of the Get3tet-luciferase complex in comparison to Get3tet complexes alone. As shown in representative micrograph images, we found that Get3tet-luciferase complexes appear larger compared to images of empty Get3tet particles (Figure 3.2.8 A). In order to visualize the complex in more detail, single particle data sets from more than 90 micrographs were collected and analyzed for common features, generating 2D reference-free projection averages. For the Get3tet-luciferase complex, the 2D averages presented in Figure 3.2.8 B show a globular arrangement with some additional density in the middle of the two lobes that form a characteristic 'W' shape in tetrameric Get3 (Figure 3.1.17 A) (Voth et al. 2014). A subset of particles showed characteristic features of empty Get3tet, confirming that our fraction contained empty Get3 tetramers in addition to the Get3tetsubstrate complexes (Figure 3.2.8 B, compare 2D averages for Get3tet and Get3tet-Luc). We aligned the reference-free 2D averages of Get3tet-lucife rase complex with the ones previously constructed from empty Get3tet, and found that the Get3-luciferase complexes indeed contain an additional density in the middle of the two 'W' shaped lobes (Figure 3.2.8 C). Unfortunately, the heterogeneity in our samples together with the limited number of particles per micrograph that correspond to a single Get3tet-luciferase complex, has made a 3D reconstruction not possible at this time. We will either have to expand our data or conduct Cryo-EM to obtain reliable 3D structures.

In summary, our in vitro results revealed that depending on its oxidation state Get3 shows low or high affinity for unfolded substrate. Once oxidized Get3 binds it substrate and maintains it in a refolding competent state. Only upon the recovery of reducing conditions, Get3 transfers its substrate to ATP-dependent foldases, which mediate the refolding, suggesting that Get3 is part of the cellular proteostasis network (Figure 4.2). We further demonstrated complex formation of Get3 with unfolded substrate in vitro, 
A

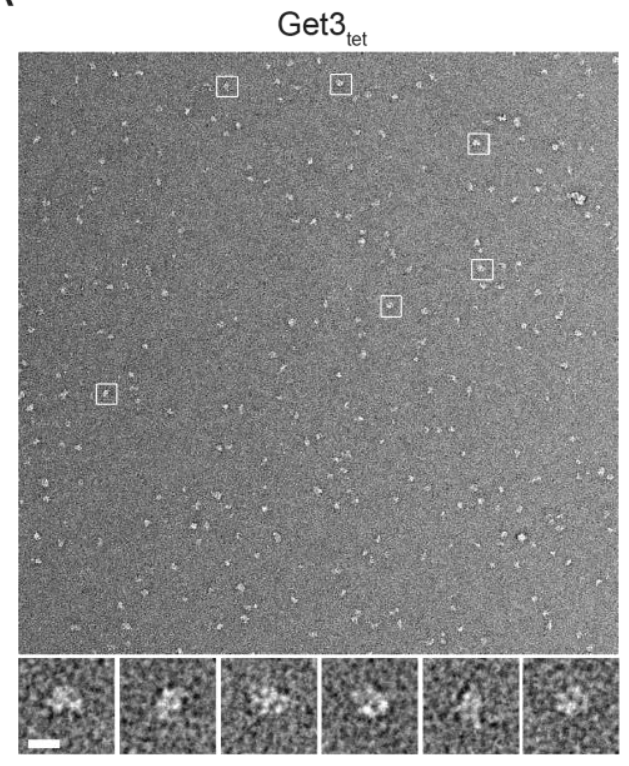

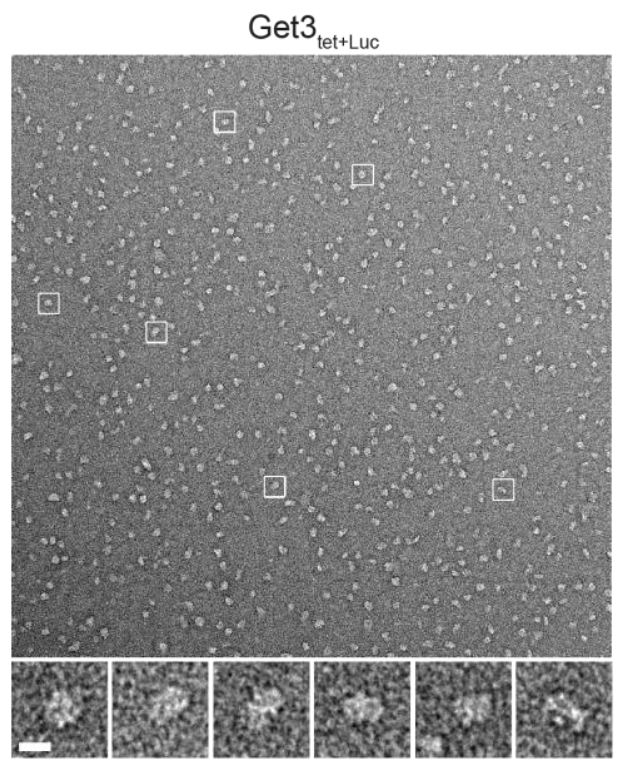

B

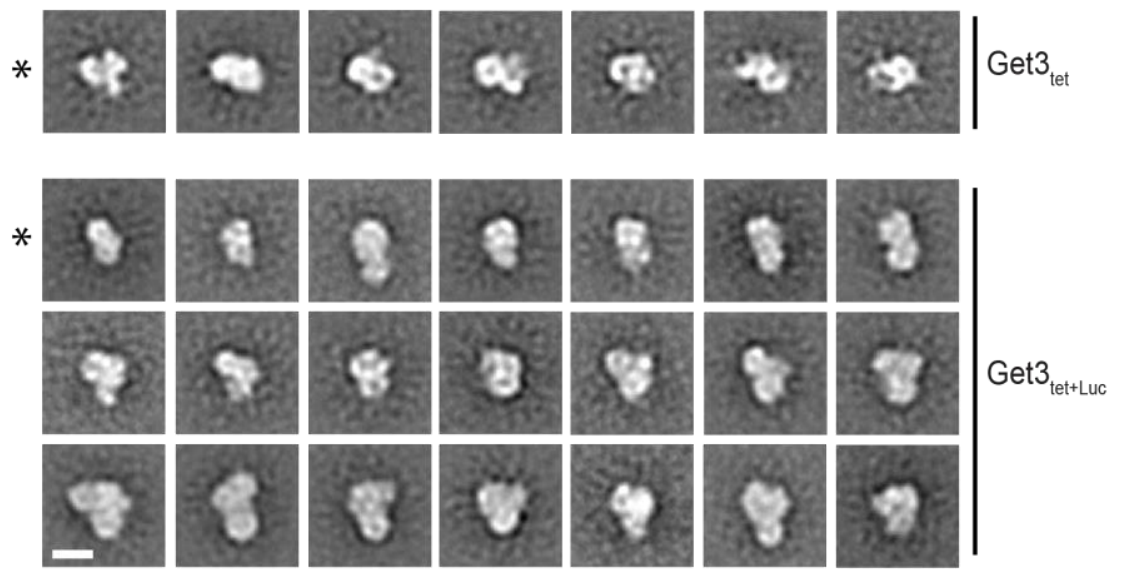

C

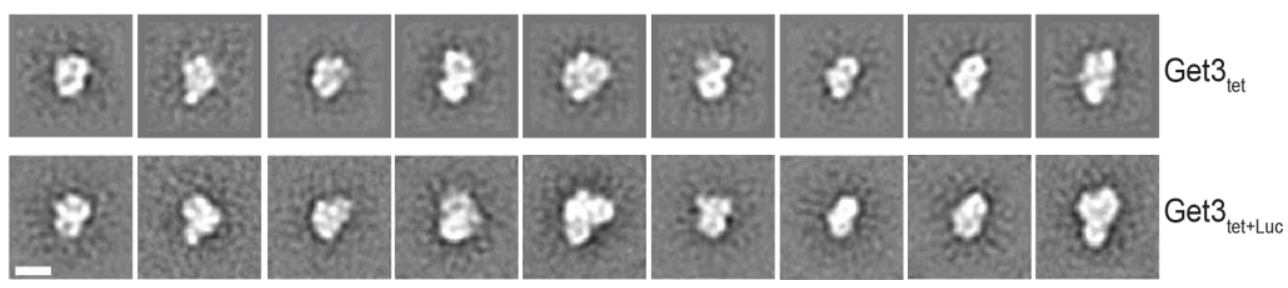

Figure 3.2. 8: Get3 $3_{\text {tet }}$-luciferase complex formation is detectible on negative stain EM (A) Peak fractions of the SEC-MALS elution for Get3 $3_{\text {tet }}$ and Get $3_{\text {tet }}$-luciferase complex were collected (Figure 3.2.7 A, light gray shaded areas) and analyzed by negative-stain electron microscopy (EM). Representative micrograph images of selected Get $3_{\text {tet }}$ (left

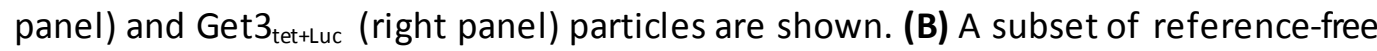
class averages for Get $3_{\text {tet }}$ al one and Get $3_{\text {tettluc }}$ complex generated using SPIDER is shown (Frank et al 1996). Class averages in the Get $3_{\text {tettluc }}$ dataset similar to the averages for Get $3_{\text {tet }}$ al one are indicated ${ }^{*}$ ) (C) A subset of reference-free Get $3_{\text {tettluc }}$ averages is aligned with averages for Get $3_{\text {tet }}$ alone, showing similar views. The scale bar equals $100 \AA$ and the box size is $260 \AA$. Imaging and analysis were carried out by Gates S (Southworth Laboratory, University of Michigan). 
however, it remains yet a subject of speculation, how the substrate is bound within the oxidized and partially disordered Get3 tetramer. 


\section{Discussion}

Living an aerobic life style is overall highly beneficial since it ensures efficient energy production. However, it comes with some undesired consequences. The respiratory chain and other enzymatic reactions in the cell constantly convert some of the oxygen into ROS, which are toxic when present at high concentrations. Therefore, dedicated antioxidant machineries are necessary for removing ROS. The delicate balance between ROS production and detoxification is disturbed, however, when metabolic rates change or when cells encounter external stressors such additional oxidants, or elevated temperatures, which not only cause protein unfolding but also lead to additional intracellular ROS accumulation.

The cellular chaperone machinery, which consists primarily of ATPdependent chaperones such as the Hsp60, 70 and 90 system, maintains protein homeostasis under most known stress conditions. Yet, this network of proteostasis factors is inefficient in compartments that lack ATP (i.e., bacterial periplasm) or when cells experience oxidative stress, which leads to a dramatic decrease in intracellular ATP levels and impairs the biogenesis of new chaperones (Colussi et al 2000, Gray et al 2014, Winter et al 2005). To functionally complement for these chaperone systems, organisms evolved a subset of posttranslationally regulated, ATP-independent chaperones, which prevent the accumulation of toxic protein aggregates (Figure 1.1). Bacteria, who regularly encounter environmental insults due to their parasitic lifestyle, developed a range of different stress-specific chaperones that are only chaperone-active when exposed to specific stress conditions, such as $\mathrm{HOCl}$ (Hsp33) or acid stress (HdeA) (Tapley et al 2009, Winter et al 2008). Over the last years, these prokaryotic stress-inducible chaperones have been extensively studied and found to constitute a crucial part of the proteostasis network. Our work on Get3 now extends the concept of stress-specific chaperones into eukaryotes, where only very few examples, such as the yeast peroxiredoxin CPrxl (Jang et al 2004) have been suggested to be posttranslationally activated as molecular chaperones. 


\subsection{Get3, a protein with a dual function as TA-protein targeting factor and general chaperone}

Get3 exhibits no significant chaperone activity under reducing non-stress conditions, and shares this feature with other known redox-regulated chaperones, such as bacterial Hsp33 (Graumann et al 2001). Instead, reduced Get3 functions as the central player of the GET pathway in yeast, mediating the post-translational insertion of TA-proteins into the ERmembrane (Schuldiner et al 2008, Simpson et al 2010, Wang et al 2010). Under these non-stress conditions, Get3 cycles between a nucleotide free open conformation with low affinity for TA-proteins and a closed, high affinity conformation upon binding of Mg-ATP (Mateja et al 2009). In the closed state, two Get3 monomers form a composite hydrophobic pocket in their $\alpha$ helical subdomains (Figure 1.7), which appears to accommodate the hydrophobic transmembrane domain of TA-proteins (Mateja et al 2015, Mateja et al 2009). The subdomain is enriched in methionines similar to the hydrophobic binding region of the signal recognition particle (SRP), and features a 20-residue insert that shields the hydrophobic pocket (TRC40insert, Figure 1.6) unique to and conserved in eukaryotic Get3 homologues (Mateja et al 2009). Interestingly, the $\alpha$-helical subdomain of Get3's prokaryotic homologue ArsA, an arsenic resistance factor, is substantially different from Get3. It misses the typical TRC40-insert and is less hydrophobic (Shen et al 2003b, Zhou et al 2000). This observation strongly suggests different molecular functions for Get3/TRC40 and ArsA.

Deletion of the mammalian Get3 homologous TRC40 or its partners is embryonically lethal (Favaloro et al 2008, Mukhopadhyay et al 2006, Stefanovic \& Hegde 2007). Although get3 deletion in yeast is not lethal, the mutants show a severe defect in a number of seemingly unrelated processes, including copper resistance (Metz et al 2006a, Shen et al 2003a), was originally attributed to a general defect in stress response due to the broad scope of TA-proteins in various cellular functions (Schuldiner et al 2008). In particular, SNARE TA-proteins, which play a key role in vesicular trafficking, are involved in metal transport and sequestration and were 


\section{Discussion}

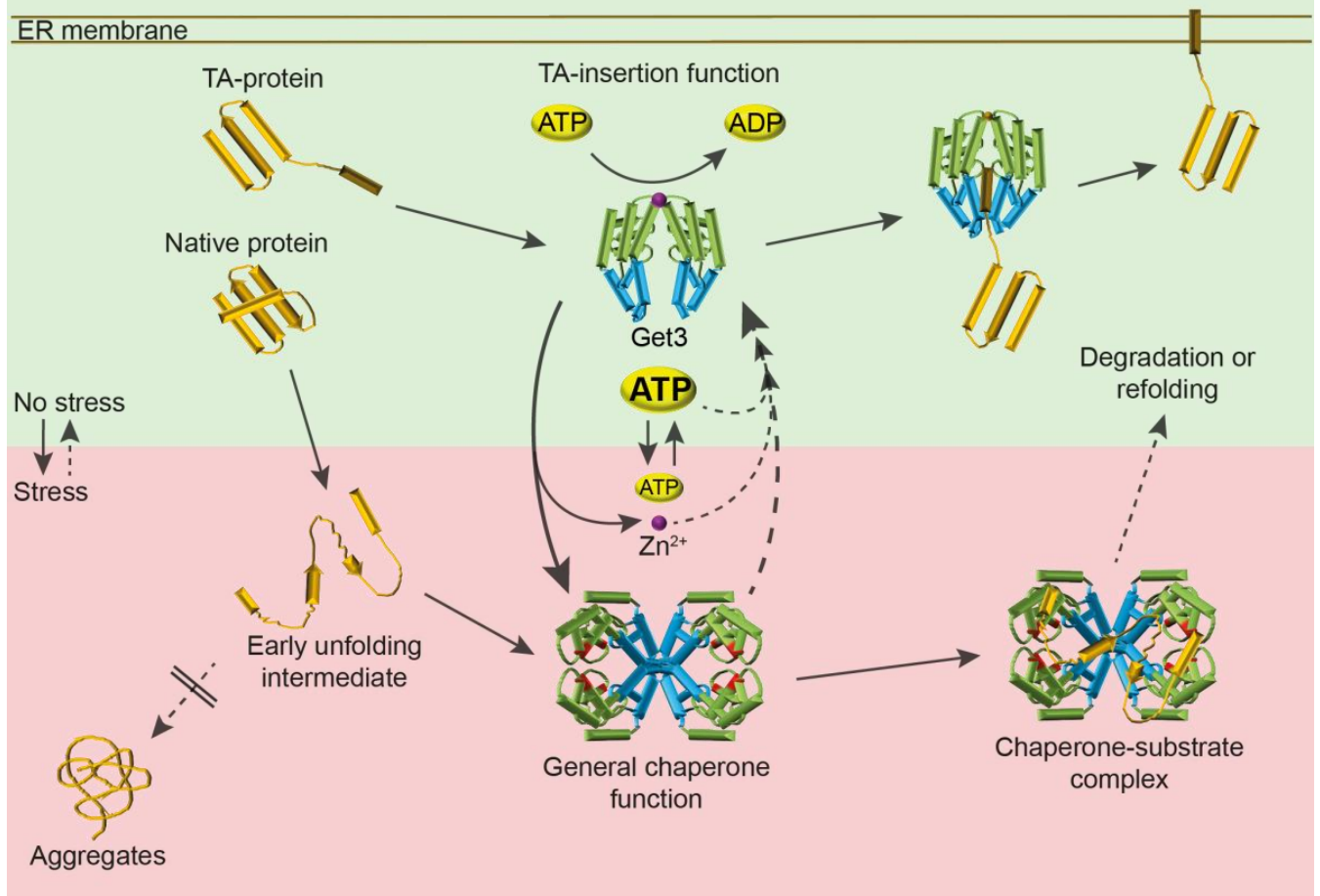

Figure 4. 1: Get3's dual function in yeast cells

Under non-stress conditions: Get3 functions as the main cytosolic component of the Guided Entry of Tail-anchored proteins (GET)-system, which supports insertion of TAproteins into the ER membrane. Homodimeric Get3 is stabilized by zinc coordination in its reduced form (magenta sphere). Binding of the transmembrane domain (brawn) of TA-clients to the composite binding site in Get3 (indicated in blue), and subsequent release on the ER membrane is regulated by ATP-binding and hydrolysis (domain indicated in green). Other cytosolic and membrane proteins involved in this process are not shown for reasons of simplicity. Upon exposure to oxidative stress conditions: Cellular ATP levels are depleted, Get3's cysteines become oxidized (marked in red) and zinc is released. Oxidation causes major conformational rearrangements, which appear to bury the TA-binding site on Get3 and turn Get3 into an ATP-independent, highly chaperone-active tetramer. Oxidized Get3 binds unfolding proteins and prevents their irreversible aggregation, protecting cells against oxidative stress. Upon return to nonstress condition and restoration of normal cellular ATP levels, Get3 returns into its initial dimer structure and presumably releases its substrate for refolding or degradation.

thought to be responsible for the phenotype (Chen et al 2001, Rees et al 2004). Our results now demonstrate that the copper sensitive phenotype of a get3 deletion strain is in fact not due to a defect in TA-targeting but due to the lack of a second function of Get3 as oxidative stress-activated, ATPindependent general chaperone (Figure 4.1).

We utilized a Get3 variant, which was defective in TA-protein targeting yet 
fully chaperone-active, and demonstrated that this mutant protein is fully able to complement the copper sensitive phenotype of a get3 deletion strain. These results unambiguously demonstrated that this phenotype is caused by the loss of the Get3 chaperone function and not by impaired TA-protein targeting.

We now propose that the chaperone function of Get3 protects cells against protein unfolding during oxidative stress conditions, either induced by exposure to exogenous ROS or endogenously elicited by ATP depletion, which causes a loss in vacuolar ATPase activity, leading to chronic oxidative stress (Milgrom et al 2007). We found that upon oxidative challenge, both wild-type Get3 and the TA-protein targeting deficient Get3 variant specifically accumulated in stress foci. These stress foci are highly similar to the Get3-containing foci that were previously observed under ATPdepletion conditions, and appear to constitute deposition sites for unfolded proteins and members of the Hsp70 and Hsp100 chaperone family (Powis et al 2013a). According to our in vitro refolding assays, which showed that Get3 is able to transfer its bound client proteins to ATP-dependent Hsp70 chaperones once non-stress conditions are restored, these results support the idea that oxidized Get3 serves as an integrated member of the cellular chaperone network. Our findings are also fully consistent with previous results on the global analysis of protein expression levels in yeast, where Get3's abundance ( 17,300 molecules) matched well with the abundance of other molecular chaperones, including Hsp104 ( 32,800 molecules) and was ten times higher than that of other members of the GET pathway, such as Get1 ( 2,250 molecules) (Ghaemmaghami et al 2003).

Our discovery that Get3 has two distinct functions in vivo is also in excellent agreement with previous findings in Caenorhabditis elegans (Hemmingsson et al 2010). In these studies, deletion of the Get3 homologue ASNA-1 was found to cause two unrelated phenotypes, a severe growth defect and increased sensitivity to the anti-cancer drug cisplatin, which is known to cause oxidative stress (Marullo et al 2013, Tsutsumishita et al 1998). 


\section{Discussion}

Intriguingly, while expression of wild-type ASNA-1 rescued both phenotypes, a ASNA-1 $22855 / \mathrm{C} 288 \mathrm{~S}$ mutant variant, which lacks two of the conserved, redox sensitive cysteines rescued the growth defect but not the cisplatin-sensitivity (Hemmingsson et al 2010). Also consistent with these results was the finding that TRC40 is massively overexpressed in breast cancer cells, in the human ovarian cancer cell line 2008 and in the human melanoma sub-line of cisplatin-resistant T289/DDP cells (Hemmingsson et al 2009a, Hemmingsson et al 2009b, Kurdi-Haidar et al 1998). Similar to ASNA-1 deletion in worms, down-regulation of TRC40 in 2008 and T289 cells increases the cisplatin sensitivity in those cancer cell lines (Hemmingsson et al 2009a, Hemmingsson et al 2009b). These results strongly suggest that it is the redox-regulated chaperone function of ASNA1/TRC40, which protects Caenorhabditis elegans and potentially cancer cells against cisplatin-mediated oxidative damage, and further indicate that ASNA/TRC40 works likely as a dual-function protein also in higher eukaryotes.

\subsection{A redox switch motif controls Get3's dual function}

Hsp33, which is highly conserved in bacteria and some unicellular eukaryotic parasites, has no known homologues in higher eukaryotes. This has raised the question as to which protein(s) take over Hsp33's functions as redox-regulated chaperone in the eukaryotic context. We now found Get3 to fulfill this role. Although there is no sequence homology between Hsp33 and Get3, many analogies can be found between the two proteins; they coordinate zinc via a conserved CXYC-motif, release zinc upon oxidative disulfide bond formation, and undergo partial unfolding events that result in an alternative structure able to act as a molecular chaperone. Reactive cysteines in redox-sensitive proteins are typically characterized by low pKa values, which allow them to be deprotonated and exhibit higher reactivity under physiological pH conditions. At least one of Get3's cysteines (Cys 285) that coordinate zinc is predicted (Dolinsky et al 2004) to have an 
unusually low $\mathrm{pKa}$ of $\sim 5$ at $\mathrm{pH} 7^{\star}$. This situation is very similar in Hsp33, where one of the four zinc binding cysteines shows a $\mathrm{pK}$ a of $\sim 5$ at $\mathrm{pH} 7^{\star *}$. We assume that like demonstrated for Hsp33, disulfide bond formation coupled to zinc release is responsible for destabilizing the domain fold in Get3 and the cause for the major unfolding events that are observed in Get3. Particularly unfolding of Get3's $\alpha$-helical ATPase domain would explain the loss of ATPase activity. Newly exposed hydrophobic residues as demonstrated by ANS binding (Figure 3.1.12) will likely serve as novel interaction sites for unfolded substrate as suggested for Hsp33 or HdeA (Groitl et al 2016, Illbert et al 2007, Tapley et al 2009). More detailed structural studies are necessary to define the exact disulfide bond connectivity in oxidized Get3, and to assess the role that intramolecular versus intermolecular disulfide bonds play in Get3ox. Segregation of the two conserved cysteine pairs in Get3 is technically challenging since deletion of either cysteine pair drastically decreases the expression and solubility of the protein. Plants, however, contain three Get3 homologues AT1, AT3 and AT5, which are located in the cytosol, chloroplast and mitochondria, respectively. Noteworthy, these three proteins differ primarily in the distribution of their cysteine pairs. Whereas cytosolic AT1 is missing the zinc-coordinating Cys 285/288 cysteine pair, AT3 and AT5 lack the Cys 240/242 pair (Figure 1.6). Preliminary studies suggest that AT1 exerts TA-protein targeting function while AT3/5 might be involved in heat sensitivity (personal correspondence with Prof. Blanche Schwappach). Since TA-proteins are only present in the outer membrane of mitochondria and chloroplast (Pedrazzini 2009), it is conceivable that AT3 and AT5 have evolved to exclusively function as chaperones in those compartments. Thus, the naturally evolved Get3 plant homologues will be subjects of further studies and will likely allow us to segregate the function of the two conserved cysteine pairs.

* Calculated with Protein Data Bank ID: $3 \mathrm{H} 84$

${ }^{* *}$ Calculated with Protein Data Bank ID: 1 XJH

Calculation for both pKa's performed with PDB2PQR Version 2.0.0 (Dolinsky et al 2004) 
Reversible disulfide bond formation accompanied with structural rearrangements is a common regulatory mechanism among various redoxactivated proteins, such as the transcriptional regulator of oxidative stress specific genes Yap1 in yeast or OxyR in E. coli. In Yap1, disulfide bond formation alters the access to the nuclear export signal motif, leading to increased nuclear localization, DNA binding and hence antioxidant gene expression (Kuge et al 2001, Kuge et al 1997). Oxidation of OxyR causes structural rearrangements in its regulatory domain and oligomerization, which support proper DNA binding and antioxidant gene expression (Choi et al 2001). In Get3, the structural rearrangements that accompany disulfide bond formation impair its ATPase activity, and mark the switch to an ATPindependent chaperone. Most importantly, the structural changes bury the alpha-helical subdomain responsible for TA-protein binding, and promote formation of higher Get3 oligomers. None of the known Get3 structures, including the tetramer formed by the archaeal Get3 homologue (Suloway et al 2012) match the structure of Get3 that we identified by EM. So far, however, Get3 was always crystallized under reducing, zinc coordinating conditions. My attempts to crystallize oxidized Get3 were not successful so far. Presumably the high heterogeneity of oligomeric Get3 and the dynamic nature of Get3's intrinsically disordered regions inhibit the nucleation process under the tested conditions. Antibodies can be employed to stabilize the fold of disordered proteins, as recently demonstrated for a disordered tau fragment (involved in Alzheimer's disease) in complex with a monoclonal antibody (Skrabana et al 2012). Hence, future crystallization approaches will involve the addition of Get3 antibodies to oligomeric Get3, which might stabilize its structure and promote the nucleation process.

Reversibility is a major aspect of redox-regulation. Upon restoration of reducing conditions and energy levels, the stress-induced holdase function of chaperones needs to be inactivated to support release and refolding of the bound client proteins. In agreement with that model, we found that the structural rearrangements and functional changes observed for Get3 upon oxidation are fully reversible, allowing Get3 to return to its ATPase dependent functions. These findings are consistent with in vivo data, which 
revealed that Get3 once colocalized with chaperones of the Hsp70 family and Hsp104 at deposition sites for aggregated proteins, can be chased out of these foci upon restoration of energy levels (Powis et al 2013a). Thus, our current working model assumes that after the stress subsides, Get3 transfers its bound substrate to the protein folding or recycling network of the cell, and then reassumes its role in protein targeting. Interestingly, while reduction of oxidized Get3 occurred within minutes in vitro, the inactivation of the chaperone function was very slow. Similar observations have previously been made for the inactivation of Hsp33 (Hoffmann et al 2004, Winter et al 2008). Since presence of Mg-ATP accelerates the conversion of Get3 to the more structured, chaperone-inactive form, we conclude that the availability of ATP in the cell rather than the end of the oxidative challenge may serve as the physiological cue that switches the Get3 chaperone off and returns the protein to the TA-protein targeting function.

\subsection{Reversible unfolding and oligomerization exposes client binding sites in chaperone active Get3}

The significant structural rearrangements that accompany Get3's oxidation process appear to bury parts of the TA-protein binding site (including the TRC40-insert, Figure 1.6 and Figure 3.1.20). This result likely excludes the possibility that the helical subdomain serves a dual function as both TAbinding and soluble client binding site, and raises the question as to where oxidized Get3 binds unfolding substrates. Chaperone-active Get3ox shares two major characteristics with other ATP-independent chaperones, such as the small Hsps (sHsps), cPrxl from yeast and mTXNPx from Leishmania infantum (Jang et al 2004, Rogalla et al 1999, Teixeira et al 2015); it contains intrinsically disordered regions and forms higher-order oligomers (Richter et al 2010, Sudnitsyna et al 2012). Chaperone active Get3 tetramers, once in complex with substrates, appear to be highly dynamic and are found to bind substrates at various molar ratios. This is similar to sHsps which form highly heterogenic complexes with unfolded substrate (Stengel et al 2010). This heterogeneity might explain the challenges that we encountered in visualizing the chaperone-substrate complexes by EM and might require 
repeated attempts using Cryo-EM.

The loss of protein structure to gain chaperone function is a fairly recently established new paradigm and has been shown for bacterial Hsp33, HdeA and small heat shock proteins (Jaya et al 2009, Reichmann et al 2012, Tapley et al 2009). The oxidation-induced decrease in the amount of secondary structure elements in Get3 are very similar to the unfolding events previously observed with Hsp33, and highly suggestive of protein unfolding. It will now remain to be seen whether the unfolded regions in Get3 contribute directly to client binding, as has been observed for Hsp33 (Reichmann et al 2012), or whether they primarily serve to increase the surface hydrophobicity in Get3.

\subsection{Get3 functions as part of a chaperone network}

ATP-independent members of the cellular chaperone network require the presence of ATP-dependent foldases for substrate refolding (Hoffmann et al 2004, Lee \& Vierling 2000, Teixeira et al 2015). Oxidized Get3, like chaperone-active Hsp33, remains in its high affinity state for unfolded substrate even beyond the restoration of reducing conditions, potentially allowing ATP-dependent chaperones time to reactivate their chaperone function (Figure 4.1) (Hoffmann et al 2004, Winter et al 2005). Upon availability of the Hsp70 folding system, substrates are released and subsequently refolded. The exact mechanism by which the Hsp70/40 system receives Get3's substrate proteins is a subject of speculation. It is feasible that Get3 releases its substrate very slowly allowing refolding by the Hsp70 system. However, we cannot exclude that the Hsp70 system directly accelerates the dissociation of reduced Get3-substrate complex by potentially supporting Get3's structural refolding (Figure 4.1).

In the context of the GET pathway in yeast, reduced Get3 forms complexes with cytosolic Get4 and Get5 further connecting it to Sgt2, which is thought to initially recognize and bind the TMD of TA-proteins and hand it over to Get3 (Wang et al 2010). At the ER-membrane, Get3 then interacts with the Get1 and Get2 receptor, which mediates the insertion of the TMD into the 
lipid bilayer (Sinning et al 2011, Stefer et al 2011). It remains unclear how the interaction with the cytosolic components Get4 and Get5 or the ER membrane receptors impact Get3's capacity to be converted to a molecular chaperone by oxidation, unfolded substrate release or the return to Get3's TA-targeting function. Get1, with its cytosolic coiled-coil domain, is thought to disrupt substrate binding in the TA-protein targeting active Get3 by penetrating the nucleotide binding domain, hence inducing conformational changes that lead to the open Get3 conformation (Stefer et al 2011). Thus, a possible involvement of Get1 in the inactivation of chaperone active Get3 or in the processing of its unfolded substrate is conceivable and needs to be evaluated in the future. Similarly, future experiments are needed to investigate whether Get3's ATPase activity potentially plays a role in its functional conversion and/or links its chaperone activity to other proteostatic mechanisms in vivo.

There are many genetic links connecting the GET pathway with major chaperone proteostasis networks (Schuldiner et al 2008). Up to know, however, these links have mainly been interpreted in light of Get3's function as a TA-protein targeting protein, which, if defective, leads to mistargeting or aggregation of TA proteins and hence affects proteostasis. However, in the context of our recent discovery that Get3 functions as a molecular chaperone specifically activated upon oxidative stress conditions, some of these interpretations need to be revisited. With our findings, we present evidence for a highly dynamic redox-regulated chaperone networks in eukaryotes (Figure 4.1 and Figure 4.2). It remains the subject of future studies to reveal how the Get3 chaperone is integrated into the cellular network that safeguards proteostasis. 


\section{Discussion}

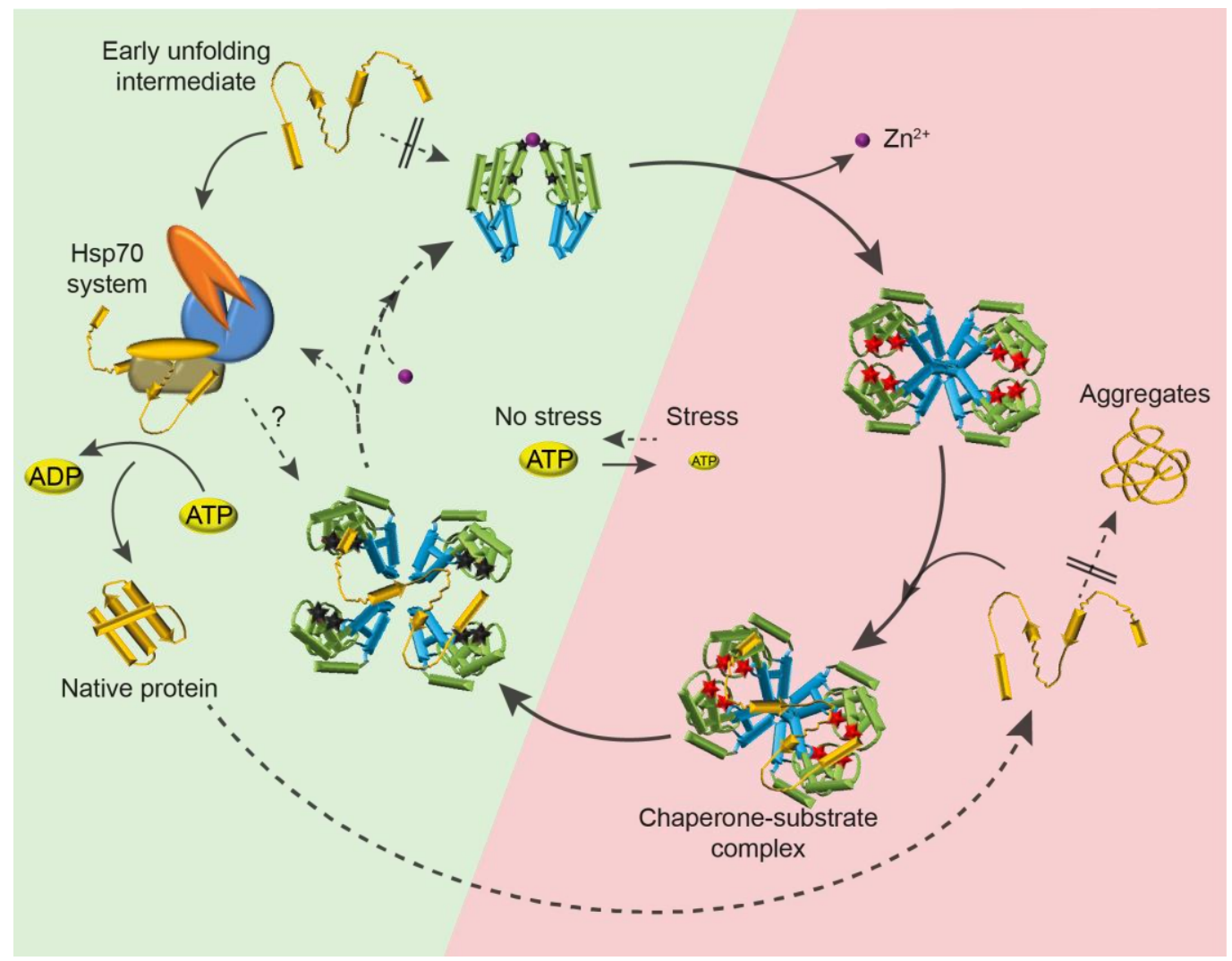

Figure 4. 2: Redox-regulated Get3 is part of the proteostasis network

The cytosolic Get3 dimer under reducing non-stressconditions, coordinates one zinc ion (magenta sphere) with two reduced cysteine pairs (black stars) from each monomer. Reduced Get3 shuttles TA-proteins in an ATP-dependent manner and shows a low affinity for general unfolded substrate. Upon exposure to oxidative stress and ATPdepleting conditions two cysteine pairs in each Get3 monomer become oxidized, leading to disulfide bonds formation (red stars), zinc release and major conformational rearrangements in Get3's ATPase domain (indicated in green). The structural changes potentially expose new binding sites for early protein unfolding intermediates, while burying the TA-binding site in the helical subdomain (blue), which now presumably serves as an oligomerization interface. The chaperone holdase active Get3 tetramer in its high affinity binding state tightly binds early unfolding protein intermediates and hence prevents their irreversible aggregation in an ATP-independent manner. Upon return to non-stress condition, Get3's cysteines are quickly reduced (black stars) disturbing the oligomerization state. Reduced Get3, however, remainschaperoneactive, holding on to its substrate. Upon recovery of cellular ATP levels, the ATP-dependent Hsp70 foldase system (KJE in prokaryotes) becomes available. Now, it either actively releases substrate from Get3 or receivesslowly released substrate, while Get3 in an ATPdependent manner gradually folds back to its dimeric structure. Substrate proteins are then refolded by the Hsp70 system. 


\section{References}

Abell BM, Pool MR, Schlenker O, Sinning I, High S. 2004. Signal recognition particle mediates post-translational targeting in eukaryotes. EMBO J 23: $2755-64$

Abell BM, Rabu C, Leznicki P, Young JC, High S. 2007. Post-translational integration of tail-anchored proteins is facilitated by defined molecular chaperones. J Cell Sci 120: 1743-51

Ahmad A, Bhattacharya A, McDonald RA, Cordes M, Ellington B, et al. 2011. Heat shock protein $70 \mathrm{kDa}$ chaperone/DnaJ cochaperone complex employs an unusual dynamic interface. Proc Natl Acad Sci U S A 108: 18966-71

Akerfelt M, Morimoto Rl, Sistonen L. 2010. Heat shock factors: integrators of cell stress, development and lifespan. Nat Rev Mol Cell Biol 11: 545-55

Ali MM, Roe SM, Vaughan CK, Meyer P, Panaretou B, et al. 2006. Crystal structure of an Hsp90-nucleotide-p23/Sba1 closed chaperone complex. Nature 440: 1013-7

Angelucci F, Saccoccia F, Ardini M, Boumis G, Brunori M, et al. 2013. Switching between the alternative structures and functions of a 2-Cys peroxiredoxin, by site-directed mutagenesis. J Mol Biol 425: 4556-68

Antelmann H, Helmann JD. 2011. Thiol-based redox switches and gene regulation. Antioxid Redox Signal 14: 1049-63

Bagneris C, Bateman OA, Naylor CE, Cronin N, Boelens WC, et al. 2009. Crystal structures of alpha-crystallin domain dimers of alphaBcrystallin and Hsp20. J Mol Biol 392: 1242-52

Bange G, Sinning I. 2013. SIMIBI twins in protein targeting and localization. Nat Struct Mol Biol 20: 776-80

Bardwell JC, Jakob U. 2012. Conditional disorder in chaperone action. Trends Biochem Sci 37: 517-25

Basha E, O'Neill H, Vierling E. 2012. Small heat shock proteins and alphacrystallins: dynamic proteins with flexible functions. Trends Biochem Sci 37: 106-17

Battle A, Jonikas MC, Walter P, Weissman JS, Koller D. 2010. Automated identification of pathways from quantitative genetic interaction data. Mol Syst Biol 6: 379

Beilharz T, Egan B, Silver PA, Hofmann K, Lithgow T. 2003. Bipartite signals mediate subcellular targeting of tail-anchored membrane proteins in Saccharomyces cerevisiae. J Biol Chem 278: 8219-23

Bertelsen EB, Chang L, Gestwicki JE, Zuiderweg ER. 2009. Solution conformation of wild-type E. coli Hsp70 (DnaK) chaperone complexed with ADP and substrate. Proc Natl Acad Sci U S A 106: 8471-6

Blacklock K, Verkhivker GM. 2014. Allosteric regulation of the Hsp90 dynamics and stability by client recruiter cochaperones: protein structure network modeling. PLoS One 9: e86547 
Borgese N, Colombo S, Pedrazzini E. 2003. The tale of tail-anchored proteins: coming from the cytosol and looking for a membrane. J Cell Biol 161: 1013-9

Bozkurt G, Stjepanovic G, Vilardi F, Amlacher S, Wild K, et al. 2009. Structural insights into tail-anchored protein binding and membrane insertion by Get3. Proc Natl Acad Sci U S A 106: 21131-6

Bracher A, Verghese J. 2015. GrpE, Hsp110/Grp170, HspBP1/Sil1 and BAG domain proteins: nucleotide exchange factors for Hsp70 molecular chaperones. Subcell Biochem 78: 1-33

Brachmann CB, Davies A, Cost GJ, Caputo E, Li J, et al. 1998. Designer deletion strains derived from Saccharomyces cerevisiae S288C: a useful set of strains and plasmids for PCR-mediated gene disruption and other applications. Yeast 14: 115-32

Brandes N, Schmitt S, Jakob U. 2009. Thiol-based redox switches in eukaryotic proteins. Antioxid Redox Signal 11: 997-1014

Brehmer D, Gassler C, Rist W, Mayer MP, Bukau B. 2004. Influence of GrpE on DnaK-substrate interactions. J Biol Chem 279: 27957-64

Broadley SA, Hartl FU. 2009. The role of molecular chaperones in human misfolding diseases. FEBS Lett 583: 2647-53

Bukau B, Weissman J, Horwich A. 2006. Molecular chaperones and protein quality control. Cell 125: 443-51

Burke D, Dawson D, Steams T. 2000. Methods in yeast genetics: a Cold Spring Harbor Laboratory course manual. Cold Spring Harbor Laboratory Press

Chae HZ, Chung SJ, Rhee SG. 1994. Thioredoxin-dependent peroxide reductase from yeast. J Biol Chem 269: 27670-8

Chang L, Bertelsen EB, Wisen S, Larsen EM, Zuiderweg ER, Gestwicki JE. 2008. High-throughput screen for small molecules that modulate the ATPase activity of the molecular chaperone DnaK. Anal Biochem 372: $167-76$

Chen YA, Scales SJ, Scheller RH. 2001. Sequential SNARE assembly underlies priming and triggering of exocytosis. Neuron 30: 161-70

Cheng G, Basha E, Wysocki VH, Vierling E. 2008. Insights into small heat shock protein and substrate structure during chaperone action derived from hydrogen/deuterium exchange and mass spectrometry. J Biol Chem 283: 26634-42

Choi H, Kim S, Mukhopadhyay P, Cho S, Woo J, et al. 2001. Structural basis of the redox switch in the OxyR transcription factor. Cell 105: 103-13

Chuang SE, Blattner FR. 1993. Characterization of twenty-six new heat shock genes of Escherichia coli. J Bacteriol 175: 5242-52

Colombo SF, Cardani S, Maroli A, Vitiello A, Soffientini P, et al. 2016. Tailanchored protein biogenesis in mammals: function and reciprocal interactions of the two subunits of the TRC40 receptor. J Biol Chem

Colussi C, Albertini MC, Coppola S, Rovidati S, Galli F, Ghibelli L. 2000. $\mathrm{H} 2 \mathrm{O} 2$-induced block of glycolysis as an active ADP-ribosylation reaction protecting cells from apoptosis. FASEB J 14: 2266-76

Cotgreave IA, Gerdes R, Schuppe-Koistinen I, Lind C. 2002. Sglutathionylation of glyceraldehyde-3-phosphate dehydrogenase: role of thiol oxidation and catalysis by glutaredoxin. Methods Enzymol 348: $175-82$ 
Cremers CM, Knoefler D, Vitvitsky V, Banerjee R, Jakob U. 2014. Bile salts act as effective protein-unfolding agents and instigators of disulfide stress in vivo. Proc Natl Acad Sci U S A 111: E1610-9

Cremers CM, Reichmann D, Hausmann J, llbert M, Jakob U. 2010. Unfolding of metastable linker region is at the core of $\mathrm{Hsp33}$ activation as a redox-regulated chaperone. J Biol Chem 285: 1124351

Daugaard M, Rohde M, Jaattela M. 2007. The heat shock protein 70 family: Highly homologous proteins with overlapping and distinct functions. FEBS Lett 581: 3702-10

Delbecq SP, Klevit RE. 2013. One size does not fit all: the oligomeric states of alphaB crystallin. FEBS Lett 587: 1073-80

Didenko T, Duarte AM, Karagoz GE, Rudiger SG. 2012. Hsp90 structure and function studied by NMR spectroscopy. Biochim Biophys Acta 1823: $636-47$

Dittmar KD, Pratt WB. 1997. Folding of the glucocorticoid receptor by the reconstituted Hsp90-based chaperone machinery. The initial hsp90.p60.hsp70-dependent step is sufficient for creating the steroid binding conformation. J Biol Chem 272: 13047-54

Dolinsky TJ, Nielsen JE, McCammon JA, Baker NA. 2004. PDB2PQR: an automated pipeline for the setup of Poisson-Boltzmann electrostatics calculations. Nucleic Acids Res 32: W665-7

Doyle SM, Genest O, Wickner S. 2013. Protein rescue from aggregates by powerful molecular chaperone machines. Nat Rev Mol Cell Biol 14: 617-29

Doyle SM, Wickner S. 2009. Hsp104 and ClpB: protein disaggregating machines. Trends Biochem Sci 34: 40-8

Drose S, Brandt U. 2012. Molecular mechanisms of superoxide production by the mitochondrial respiratory chain. Adv Exp Med Biol 748: 14569

Ellis RJ. 2006. Molecular chaperones: assisting assembly in addition to folding. Trends Biochem Sci 31: 395-401

Englander SW. 2000. Protein folding intermediates and pathways studied by hydrogen exchange. Annu Rev Biophys Biomol Struct 29: 213-38

Fan CY, Lee S, Ren HY, Cyr DM. 2004. Exchangeable chaperone modules contribute to specification of type I and type II Hsp40 cellular function. Mol Biol Cell 15: 761-73

Favaloro V, Spasic M, Schwappach B, Dobberstein B. 2008. Distinct targeting pathways for the membrane insertion of tail-anchored (TA) proteins. Journal of Cell Science 121: 1832-40

Finka A, Goloubinoff P. 2013. Proteomic data from human cell cultures refine mechanisms of chaperone-mediated protein homeostasis. Cell Stress Chaperones 18: 591-605

Fodje MN, Hansson A, Hansson M, Olsen JG, Gough S, et al. 2001. Interplay between an AAA module and an integrin I domain may regulate the function of magnesium chelatase. J Mol Biol 311: 11122

Frank J, Radermacher M, Penczek P, Zhu J, Li Y, et al. 1996. SPIDER and WEB: processing and visualization of images in 3D electron microscopy and related fields. J Struct Biol 116: 190-9 
Franzmann TM, Wuhr M, Richter K, Walter S, Buchner J. 2005. The activation mechanism of Hsp26 does not require dissociation of the oligomer. J Mol Biol 350: 1083-93

Ghaemmaghami S, Huh WK, Bower K, Howson RW, Belle A, et al. 2003. Global analysis of protein expression in yeast. Nature 425: 737-41

Goodson ML, Sarge KD. 1995. Heat-inducible DNA binding of purified heat shock transcription factor 1. J Biol Chem 270: 2447-50

Graf PC, Martinez-Yamout M, VanHaerents S, Lilie H, Dyson HJ, Jakob U. 2004. Activation of the redox-regulated chaperone Hsp33 by domain unfolding. J Biol Chem 279: 20529-38

Graumann J, Lilie H, Tang X, Tucker KA, Hoffmann JH, et al. 2001. Activation of the redox-regulated molecular chaperone Hsp33--a twostep mechanism. Structure 9: 377-87

Gray MJ, Wholey WY, Wagner NO, Cremers CM, Mueller-Schickert A, et al. 2014. Polyphosphate is a primordial chaperone. Mol Cell 53: 689-99

Greene MK, Maskos K, Landry SJ. 1998. Role of the J-domain in the cooperation of Hsp40 with Hsp70. Proc Natl Acad Sci U S A 95: 610813

Greenfield NJ. 2006. Using circular dichroism spectra to estimate protein secondary structure. Nat Protoc 1: 2876-90

Gribun A, Cheung KL, Huen J, Ortega J, Houry WA. 2008. Yeast Rvb1 and Rvb2 are ATP-dependent DNA helicases that form a heterohexameric complex. J Mol Biol 376: 1320-33

Groitl B, Horowitz S, Makepeace KA, Petrotchenko EV, Borchers CH, et al. 2016. Protein unfolding as a switch from self-recognition to highaffinity client binding. Nat Commun 7: 10357

Groitl B, Jakob U. 2014. Thiol-based redox switches. Biochim Biophys Acta 1844: 1335-43

Guisbert E, Herman C, Lu CZ, Gross CA. 2004. A chaperone network controls the heat shock response in E. coli. Genes Dev 18: 2812-21

Hageman J, Kampinga HH. 2009. Computational analysis of the human HSPH/HSPA/DNAJ family and cloning of a human HSPH/HSPA/DNAJ expression library. Cell Stress Chaperones 14: $1-21$

Harrison C. 2003. GrpE, a nucleotide exchange factor for DnaK. Cell Stress Chaperones 8: 218-24

Hartl FU. 2011. Chaperone-assisted protein folding: the path to discovery from a personal perspective. Nat Med 17: 1206-10

Hartl FU, Bracher A, Hayer-Hartl M. 2011. Molecular chaperones in protein folding and proteostasis. Nature 475: 324-32

Haslbeck M, Braun N, Stromer T, Richter B, Model N, et al. 2004. Hsp42 is the general small heat shock protein in the cytosol of Saccharomyces cerevisiae. EMBO J 23: 638-49

Haslbeck M, Franzmann T, Weinfurtner D, Buchner J. 2005a. Some like it hot: the structure and function of small heat-shock proteins. Nat Struct Mol Biol 12: 842-6

Haslbeck M, Miess A, Stromer T, Walter S, Buchner J. 2005b. Disassembling protein aggregates in the yeast cytosol. The cooperation of Hsp26 with Ssa1 and Hsp104. J Biol Chem 280: 23861-8 
Haslbeck M, Vierling E. 2015. A first line of stress defense: small heat shock proteins and their function in protein homeostasis. J Mol Biol 427: 1537-48

Haslbeck M, Walke S, Stromer T, Ehrnsperger M, White HE, et al. 1999. Hsp26: a temperature-regulated chaperone. EMBO J 18: 6744-51

Hemmingsson O, Kao G, Still M, Naredi P. 2010. ASNA-1 activity modulates sensitivity to cisplatin. Cancer Res 70: 10321-8

Hemmingsson O, Nojd M, Kao G, Naredi P. 2009a. Increased sensitivity to platinating agents and arsenite in human ovarian cancer by downregulation of ASNA1. Oncol Rep 22: 869-75

Hemmingsson O, Zhang Y, Still M, Naredi P. 2009b. ASNA1, an ATPase targeting tail-anchored proteins, regulates melanoma cell growth and sensitivity to cisplatin and arsenite. Cancer Chemother Pharmacol 63: 491-9

Herbst R, Schafer U, Seckler R. 1997. Equilibrium intermediates in the reversible unfolding of firefly (Photinus pyralis) luciferase. $J \mathrm{Biol}$ Chem 272: 7099-105

Hirotsu S, Abe Y, Okada K, Nagahara N, Hori H, et al. 1999. Crystal structure of a multifunctional 2-Cys peroxiredoxin heme-binding protein $23 \mathrm{kDa}$ /proliferation-associated gene product. Proc Natl Acad Sci U S A 96: 12333-8

Hoffmann JH, Linke K, Graf PC, Lilie H, Jakob U. 2004. Identification of a redox-regulated chaperone network. EMBO J 23: 160-8

Holmberg Cl, Hietakangas V, Mikhailov A, Rantanen JO, Kallio M, et al. 2001. Phosphorylation of serine 230 promotes inducible transcriptional activity of heat shock factor 1. EMBO J 20: 3800-10

Horwich AL, Farr GW, Fenton WA. 2006. GroEL-GroES-mediated protein folding. Chem Rev 106: 1917-30

Horwich AL, Fenton WA, Chapman E, Farr GW. 2007. Two families of chaperonin: physiology and mechanism. Annu Rev Cell Dev Biol 23: 115-45

Hu J, Li J, Qian X, Denic V, Sha B. 2009. The crystal structures of yeast Get3 suggest a mechanism for tail-anchored protein membrane insertion. PLoS One 4: e8061

Hunt JF, Weaver AJ, Landry SJ, Gierasch L, Deisenhofer J. 1996. The crystal structure of the GroES co-chaperonin at 2.8 A resolution. Nature 379: 37-45

Ilbert M, Horst J, Ahrens S, Winter J, Graf PC, et al. 2007. The redox-switch domain of Hsp33 functions as dual stress sensor. Nat Struct Mol Biol 14: $556-63$

Imlay JA. 2013. The molecular mechanisms and physiological consequences of oxidative stress: lessons from a model bacterium. Nat Rev Microbiol 11: 443-54

Ito $\mathrm{H}$, Fukuda Y, Murata K, Kimura A. 1983. Transformation of intact yeast cells treated with alkali cations. J Bacteriol 153: 163-8

Jahn TR, Radford SE. 2008. Folding versus aggregation: polypeptide conformations on competing pathways. Arch Biochem Biophys 469: 100-17

Jakob U, Eser M, Bardwell JC. 2000. Redox switch of hsp33 has a novel zinc-binding motif. J Biol Chem 275: 38302-10 
Jakob U, Kriwacki R, Uversky VN. 2014. Conditionally and transiently disordered proteins: awakening cryptic disorder to regulate protein function. Chem Rev 114: 6779-805

Jakob U, Lilie H, Meyer I, Buchner J. 1995. Transient interaction of Hsp90 with early unfolding intermediates of citrate synthase. Implications for heat shock in vivo. J Biol Chem 270: 7288-94

Jakob U, Muse W, Eser M, Bardwell JC. 1999. Chaperone activity with a redox switch. Cell 96: 341-52

Janda I, Devedjiev Y, Derewenda U, Dauter Z, Bielnicki J, et al. 2004. The crystal structure of the reduced, Zn2+-bound form of the B. subtilis Hsp33 chaperone and its implications for the activation mechanism. Structure 12: 1901-7

Jang HH, Kim SY, Park SK, Jeon HS, Lee YM, et al. 2006. Phosphorylation and concomitant structural changes in human 2-Cys peroxiredoxin isotype I differentially regulate its peroxidase and molecular chaperone functions. FEBS Lett 580: 351-5

Jang HH, Lee KO, Chi YH, Jung BG, Park SK, et al. 2004. Two enzymes in one; two yeast peroxiredoxins display oxidative stress-dependent switching from a peroxidase to a molecular chaperone function. Cell 117: 625-35

Jaya N, Garcia V, Vierling E. 2009. Substrate binding site flexibility of the small heat shock protein molecular chaperones. Proc Natl Acad Sci U S A 106: 15604-9

Jehle S, Vollmar BS, Bardiaux B, Dove KK, Rajagopal P, et al. 2011. Nterminal domain of alphaB-crystallin provides a conformational switch for multimerization and structural heterogeneity. Proc Natl Acad Sci U S A 108: 6409-14

Jonikas MC, Collins SR, Denic V, Oh E, Quan EM, et al. 2009. Comprehensive characterization of genes required for protein folding in the endoplasmic reticulum. Science 323: 1693-7

Jung T, Grune T. 2013. The proteasome and the degradation of oxidized proteins: Part I-structure of proteasomes. Redox Biol 1: 178-82

Jung T, Hohn A, Grune T. 2013. The proteasome and the degradation of oxidized proteins: Part II - protein oxidation and proteasomal degradation. Redox Biol 2C: 99-104

Kachur AV, Koch CJ, Biaglow JE. 1999. Mechanism of copper-catalyzed autoxidation of cysteine. Free Radic Res 31: 23-34

Kalbfleisch T, Cambon A, Wattenberg BW. 2007. A bioinformatics approach to identifying tail-anchored proteins in the human genome. Traffic 8: 1687-94

Kampinga HH, Craig EA. 2010. The HSP70 chaperone machinery: J proteins as drivers of functional specificity. Nat Rev Mol Cell Biol 11: $579-92$

Kan ZY, Walters BT, Mayne L, Englander SW. 2013. Protein hydrogen exchange at residue resolution by proteolytic fragmentation mass spectrometry analysis. Proc Natl Acad Sci U S A 110: 16438-43

Kiianitsa K, Solinger JA, Heyer WD. 2003. NADH-coupled microplate photometric assay for kinetic studies of ATP-hydrolyzing enzymes with low and high specific activities. Anal Biochem 321: 266-71 
Klebanoff SJ. 2005. Myeloperoxidase: friend and foe. J Leukoc Biol 77: 598625

Kohl C, Tessarz P, von der Malsburg K, Zahn R, Bukau B, Mogk A. 2011. Cooperative and independent activities of Sgt2 and Get5 in the targeting of tail-anchored proteins. Biol Chem 392: 601-8

Kriechbaumer V, Shaw R, Mukherjee J, Bowsher CG, Harrison AM, Abell BM. 2009. Subcellular distribution of tail-anchored proteins in Arabidopsis. Traffic 10: 1753-64

Kriehuber T, Rattei T, Weinmaier T, Bepperling A, Haslbeck M, Buchner J. 2010. Independent evolution of the core domain and its flanking sequences in small heat shock proteins. FASEB J 24: 3633-42

Krukenberg KA, Bottcher UM, Southworth DR, Agard DA. 2009. Grp94, the endoplasmic reticulum Hsp90, has a similar solution conformation to cytosolic Hsp90 in the absence of nucleotide. Protein Sci 18: 181527

Kuge S, Arita M, Murayama A, Maeta K, lzawa S, et al. 2001. Regulation of the yeast Yap1p nuclear export signal is mediated by redox signalinduced reversible disulfide bond formation. Mol Cell Biol 21: 613950

Kuge S, Jones N, Nomoto A. 1997. Regulation of yAP-1 nuclear localization in response to oxidative stress. EMBO J 16: 1710-20

Kumar DP, Vorvis C, Sarbeng EB, Cabra Ledesma VC, Willis JE, Liu Q. 2011. The four hydrophobic residues on the Hsp70 inter-domain linker have two distinct roles. J Mol Biol 411: 1099-113

Kumsta C, Jakob U. 2009. Redox-regulated chaperones. Biochemistry 48: 4666-76

Kurdi-Haidar B, Heath D, Naredi P, Varki N, Howell SB. 1998. Immunohistochemical analysis of the distribution of the human ATPase (hASNA-I) in normal tissues and its overexpression in breast adenomas and carcinomas. J Histochem Cytochem 46: 1243-8

Le Moan N, Clement G, Le Maout S, Tacnet F, Toledano MB. 2006. The Saccharomyces cerevisiae proteome of oxidized protein thiols: contrasted functions for the thioredoxin and glutathione pathways. $J$ Biol Chem 281: 10420-30

Lee GJ, Vierling E. 2000. A small heat shock protein cooperates with heat shock protein 70 systems to reactivate a heat-denatured protein. Plant Physiol 122: 189-98

Lee S, Sielaff B, Lee J, Tsai FT. 2010. CryoEM structure of Hsp104 and its mechanistic implication for protein disaggregation. Proc Natl Acad Sci U S A 107: 8135-40

Lee S, Sowa ME, Watanabe YH, Sigler PB, Chiu W, et al. 2003. The structure of ClpB: a molecular chaperone that rescues proteins from an aggregated state. Cell 115: 229-40

Leichert LI, Gehrke F, Gudiseva HV, Blackwell T, llbert M, et al. 2008. Quantifying changes in the thiol redox proteome upon oxidative stress in vivo. Proc Natl Acad Sci U S A 105: 8197-202

Lennon CW, Thamsen M, Friman ET, Cacciaglia A, Sachsenhauser V, et al. 2015. Folding Optimization In Vivo Uncovers New Chaperones. J Mol Biol 427: 2983-94 
Liberek K, Lewandowska A, Zietkiewicz S. 2008. Chaperones in control of protein disaggregation. EMBO J 27: 328-35

Lin J, Lee IS, Frey J, Slonczewski JL, Foster JW. 1995. Comparative analysis of extreme acid survival in Salmonella typhimurium, Shigella flexneri, and Escherichia coli. J Bacteriol 177: 4097-104

Llorca O, Smyth MG, Carrascosa JL, Willison KR, Radermacher M, et al. 1999. 3D reconstruction of the ATP-bound form of CCT reveals the asymmetric folding conformation of a type II chaperonin. Nat Struct Biol 6: 639-42

Mally A, Witt SN. 2001. GrpE accelerates peptide binding and release from the high affinity state of DnaK. Nat Struct Biol 8: 254-7

Maret W. 2006. Zinc coordination environments in proteins as redox sensors and signal transducers. Antioxid Redox Signal 8: 1419-41

Mariappan M, Li X, Stefanovic S, Sharma A, Mateja A, et al. 2010. A ribosome-associating factor chaperones tail-anchored membrane proteins. Nature 466: 1120-4

Maritim AC, Sanders RA, Watkins JB, 3rd. 2003. Diabetes, oxidative stress, and antioxidants: a review. J Biochem Mol Toxicol 17: 24-38

Marsh JJ, Guan HS, Li S, Chiles PG, Tran D, Morris TA. 2013. Structural insights into fibrinogen dynamics using amide hydrogen/deuterium exchange mass spectrometry. Biochemistry 52: 5491-502

Marullo R, Werner E, Degtyareva N, Moore B, Altavilla G, et al. 2013. Cisplatin induces a mitochondrial-ROS response that contributes to cytotoxicity depending on mitochondrial redox status and bioenergetic functions. PLoS One 8: e81162

Mateja A, Paduch M, Chang HY, Szydlowska A, Kossiakoff AA, et al. 2015. Protein targeting. Structure of the Get3 targeting factor in complex with its membrane protein cargo. Science 347: 1152-5

Mateja A, Szlachcic A, Downing ME, Dobosz M, Mariappan M, et al. 2009. The structural basis of tail-anchored membrane protein recognition by Get3. Nature 461: 361-6

Matsui M, Takaya A, Yamamoto T. 2008. Sigma32-mediated negative regulation of Salmonella pathogenicity island 1 expression. $J$ Bacteriol 190: 6636-45

Matsumoto R, Akama K, Rakwal R, Iwahashi H. 2005. The stress response against denatured proteins in the deletion of cytosolic chaperones SSA1/2 is different from heat-shock response in Saccharomyces cerevisiae. BMC Genomics 6: 141

Matuszewska E, Kwiatkowska J, Ratajczak E, Kuczynska-Wisnik D, Laskowska E. 2009. Role of Escherichia coli heat shock proteins IbpA and $\mathrm{lbpB}$ in protection of alcohol dehydrogenase AdhE against heat inactivation in the presence of oxygen. Acta Biochim Pol 56: 55-61

Mayer MP. 2013. Hsp70 chaperone dynamics and molecular mechanism. Trends Biochem Sci 38: 507-14

Mayer MP, Brehmer D, Gassler CS, Bukau B. 2001. Hsp70 chaperone machines. Adv Protein Chem 59: 1-44

Mayer MP, Laufen T, Paal K, McCarty JS, Bukau B. 1999. Investigation of the interaction between DnaK and DnaJ by surface plasmon resonance spectroscopy. J Mol Biol 289: 1131-44 
Metz J, Wachter A, Schmidt B, Bujnicki JM, Schwappach B. 2006a. The yeast Arr4p ATPase binds the chloride transporter Gef1p when copper is available in the cytosol. The Journal of biological chemistry 281: 410-7

Metz J, Wachter A, Schmidt B, Bujnicki JM, Schwappach B. 2006b. The yeast Arr4p ATPase binds the chloride transporter Gef1p when copper is available in the cytosol. J Biol Chem 281: 410-7

Milgrom E, Diab H, Middleton F, Kane PM. 2007. Loss of vacuolar protontranslocating ATPase activity in yeast results in chronic oxidative stress. J Biol Chem 282: 7125-36

Miller RA, Britigan BE. 1997. Role of oxidants in microbial pathophysiology. Clin Microbiol Rev 10: 1-18

Mistry R, Cliff JM, Clayton CL, Beyers N, Mohamed YS, et al. 2007. Geneexpression patterns in whole blood identify subjects at risk for recurrent tuberculosis. J Infect Dis 195: 357-65

Miyata Y, Yahara I. 1995. Interaction between casein kinase II and the 90$\mathrm{kDa}$ stress protein, HSP90. Biochemistry 34: 8123-9

Morano KA, Grant CM, Moye-Rowley WS. 2012. The response to heat shock and oxidative stress in Saccharomyces cerevisiae. Genetics 190: 1157-95

Morita MT, Tanaka Y, Kodama TS, Kyogoku Y, Yanagi H, Yura T. 1999. Translational induction of heat shock transcription factor sigma32: evidence for a built-in RNA thermosensor. Genes Dev 13: 655-65

Mukhopadhyay R, Ho YS, Swiatek PJ, Rosen BP, Bhattacharjee H. 2006. Targeted disruption of the mouse Asna1 gene results in embryonic lethality. FEBS Lett 580: 3889-94

Muller A, Langklotz S, Lupilova N, Kuhlmann K, Bandow JE, Leichert LI. 2014. Activation of RidA chaperone function by N-chlorination. Nat Commun 5: 5804

Nakahigashi K, Ron EZ, Yanagi H, Yura T. 1999. Differential and independent roles of a sigma(32) homolog $(\mathrm{RpoH})$ and an $\mathrm{HrcA}$ repressor in the heat shock response of Agrobacterium tumefaciens. $J$ Bacteriol 181: 7509-15

Nesterenko MV, Tilley M, Upton SJ. 1994. A simple modification of Blum's silver stain method allows for 30 minute detection of proteins in polyacrylamide gels. J Biochem Biophys Methods 28: 239-42

Nonaka G, Blankschien M, Herman C, Gross CA, Rhodius VA. 2006. Regulon and promoter analysis of the $\mathrm{E}$. coli heat-shock factor, sigma32, reveals a multifaceted cellular response to heat stress. Genes Dev 20: 1776-89

Norby JG. 1988. Coupled assay of $\mathrm{Na}+, \mathrm{K}+-\mathrm{ATPase}$ activity. Methods Enzymol 156: 116-9

Ohi M, Li Y, Cheng Y, Walz T. 2004. Negative Staining and Image Classification - Powerful Tools in Modern Electron Microscopy. Biol Proced Online 6: 23-34

Osorio $\mathrm{H}$, Carvalho E, del Valle M, Gunther Sillero MA, Moradas-Ferreira P, Sillero A. 2003. $\mathrm{H} 2 \mathrm{O} 2$, but not menadione, provokes a decrease in the ATP and an increase in the inosine levels in Saccharomyces cerevisiae. An experimental and theoretical approach. Eur J Biochem 270: $1578-89$ 
Packschies L, Theyssen H, Buchberger A, Bukau B, Goody RS, Reinstein J. 1997. GrpE accelerates nucleotide exchange of the molecular chaperone DnaK with an associative displacement mechanism. Biochemistry 36: 3417-22

Patel S, Vierling E, Tama F. 2014. Replica exchange molecular dynamics simulations provide insight into substrate recognition by small heat shock proteins. Biophys J 106: 2644-55

Pattison DI, Davies MJ. 2006. Reactions of myeloperoxidase-derived oxidants with biological substrates: gaining chemical insight into human inflammatory diseases. Curr Med Chem 13: 3271-90

Pearl LH, Prodromou C, Workman P. 2008. The Hsp90 molecular chaperone: an open and shut case for treatment. Biochem $J 410$ : 439-53

Pecci L, Montefoschi G, Cavallini D. 1997. Some new details of the copperhydrogen peroxide interaction. Biochem Biophys Res Commun 235: 264-7

Pedrazzini E. 2009. Tail-Anchored Proteins in Plants. Journal of Plant Biology 52: 88-101

Pereira JH, Ralston CY, Douglas NR, Meyer D, Knee KM, et al. 2010. Crystal structures of a group II chaperonin reveal the open and closed states associated with the protein folding cycle. J Biol Chem 285: 27958-66

Petrucelli L, Dickson D, Kehoe K, Taylor J, Snyder H, et al. 2004. CHIP and Hsp70 regulate tau ubiquitination, degradation and aggregation. Hum Mol Genet 13: 703-14

Plater ML, Goode D, Crabbe MJ. 1996. Effects of site-directed mutations on the chaperone-like activity of alphaB-crystallin. J Biol Chem 271: 28558-66

Poole LB, Hall A, Nelson KJ. 2011. Overview of peroxiredoxins in oxidant defense and redox regulation. Curr Protoc Toxicol Chapter 7: Unit7 9

Popp S, Packschies L, Radzwill N, Vogel KP, Steinhoff HJ, Reinstein J. 2005. Structural dynamics of the DnaK-peptide complex. J Mol Biol 347: $1039-52$

Poulain P, Gelly JC, Flatters D. 2010. Detection and architecture of small heat shock protein monomers. PLoS One 5: e9990

Powis K, Schrul B, Tienson H, Gostimskaya I, Breker M, et al. 2013a. Get3 is a holdase chaperone and moves to deposition sites for aggregated proteins when membrane targeting is blocked. Journal of Cell Science 126: 473-83

Powis K, Schrul B, Tienson H, Gostimskaya I, Breker M, et al. 2013b. Get3 is a holdase chaperone and moves to deposition sites for aggregated proteins when membrane targeting is blocked. J Cell Sci 126: 473-83

Quan S, Koldewey P, Tapley T, Kirsch N, Ruane KM, et al. 2011. Genetic selection designed to stabilize proteins uncovers a chaperone called Spy. Nat Struct Mol Biol 18: 262-9

Rabu C, Schmid V, Schwappach B, High S. 2009. Biogenesis of tailanchored proteins: the beginning for the end? J Cell Sci 122: 360512 
Rabu C, Wipf P, Brodsky JL, High S. 2008. A precursor-specific role for Hsp40/Hsc70 during tail-anchored protein integration at the endoplasmic reticulum. J Biol Chem 283: 27504-13

Radermacher M, Wagenknecht T, Verschoor A, Frank J. 1987. Threedimensional reconstruction from a single-exposure, random conical tilt series applied to the $50 \mathrm{~S}$ ribosomal subunit of Escherichia coli. $J$ Microsc 146: 113-36

Rajan VB, D'Silva P. 2009. Arabidopsis thaliana J-class heat shock proteins: cellular stress sensors. Funct Integr Genomics 9: 433-46

Rees EM, Lee J, Thiele DJ. 2004. Mobilization of intracellular copper stores by the ctr2 vacuolar copper transporter. J Biol Chem 279: 54221-9

Reichmann D, Xu Y, Cremers CM, llbert M, Mittelman R, et al. 2012. Order out of disorder: working cycle of an intrinsically unfolded chaperone. Cell 148: 947-57

Reis A, Fonseca C, Maciel E, Domingues P, Domingues MR. 2011. Influence of amino acid relative position on the oxidative modification of histidine and glycine peptides. Anal Bioanal Chem 399: 2779-94

Rhee SG, Chae HZ, Kim K. 2005. Peroxiredoxins: a historical overview and speculative preview of novel mechanisms and emerging concepts in cell signaling. Free Radic Biol Med 38: 1543-52

Rhee SG, Woo HA. 2011. Multiple functions of peroxiredoxins: peroxidases, sensors and regulators of the intracellular messenger $\mathrm{H}(2) \mathrm{O}(2)$, and protein chaperones. Antioxid Redox Signal 15: 781-94

Richter K, Haslbeck M, Buchner J. 2010. The heat shock response: life on the verge of death. Mol Cell 40: 253-66

Riddles PW, Blakeley RL, Zerner B. 1983. Reassessment of Ellman's reagent. Methods Enzymol 91: 49-60

Rogalla T, Ehrnsperger M, Preville X, Kotlyarov A, Lutsch G, et al. 1999. Regulation of Hsp27 oligomerization, chaperone function, and protective activity against oxidative stress/tumor necrosis factor alpha by phosphorylation. J Biol Chem 274: 18947-56

Romano AD, Serviddio G, de Matthaeis A, Bellanti F, Vendemiale G. 2010. Oxidative stress and aging. J Nephrol 23 Suppl 15: S29-36

Rome ME, Chio US, Rao M, Gristick H, Shan SO. 2014. Differential gradients of interaction affinities drive efficient targeting and recycling in the GET pathway. Proc Natl Acad Sci U S A 111: E4929-35

Rome ME, Rao M, Clemons WM, Shan SO. 2013. Precise timing of ATPase activation drives targeting of tail-anchored proteins. Proc Natl Acad Sci U S A 110: 7666-71

Roth DM, Balch WE. 2011. Modeling general proteostasis: proteome balance in health and disease. Curr Opin Cell Biol 23: 126-34

Rowley A, Johnston GC, Butler B, Werner-Washburne M, Singer RA. 1993. Heat shock-mediated cell cycle blockage and G1 cyclin expression in the yeast Saccharomyces cerevisiae. Mol Cell Biol 13: 1034-41

Rudiger S, Germeroth L, Schneider-Mergener J, Bukau B. 1997. Substrate specificity of the DnaK chaperone determined by screening cellulosebound peptide libraries. EMBO J 16: 1501-7

Rye HS, Roseman AM, Chen S, Furtak K, Fenton WA, et al. 1999. GroEL GroES cycling: ATP and nonnative polypeptide direct alternation of folding-active rings. Cell 97: 325-38 
Saccoccia F, Di Micco P, Boumis G, Brunori M, Koutris I, et al. 2012. Moonlighting by different stressors: crystal structure of the chaperone species of a 2-Cys peroxiredoxin. Structure 20: 429-39

Sakurai $\mathrm{H}$, Takemori Y. 2007. Interaction between heat shock transcription factors (HSFs) and divergent binding sequences: binding specificities of yeast HSFs and human HSF1. J Biol Chem 282: 13334-41

Sauer RT, Bolon DN, Burton BM, Burton RE, Flynn JM, et al. 2004. Sculpting the proteome with $\mathrm{AAA}(+)$ proteases and disassembly machines. Cell 119: $9-18$

Scheres SH. 2012. RELION: implementation of a Bayesian approach to cryo-EM structure determination. J Struct Biol 180: 519-30

Schlieker C, Tews I, Bukau B, Mogk A. 2004. Solubilization of aggregated proteins by ClpB/DnaK relies on the continuous extraction of unfolded polypeptides. FEBS Lett 578: 351-6

Schroder E, Littlechild JA, Lebedev AA, Errington N, Vagin AA, Isupov MN. 2000. Crystal structure of decameric 2-Cys peroxiredoxin from human erythrocytes at 1.7 A resolution. Structure 8: 605-15

Schroder H, Langer T, Hartl FU, Bukau B. 1993. DnaK, DnaJ and GrpE form a cellular chaperone machinery capable of repairing heat-induced protein damage. EMBO J 12: 4137-44

Schuldiner M, Metz J, Schmid V, Denic V, Rakwalska M, et al. 2008. The GET complex mediates insertion of tail-anchored proteins into the ER membrane. Cell 134: 634-45

Shao S, Hegde RS. 2011. Membrane protein insertion at the endoplasmic reticulum. Annu Rev Cell Dev Biol 27: 25-56

Shen J, Hsu CM, Kang BK, Rosen BP, Bhattacharjee H. 2003a. The Saccharomyces cerevisiae Arr $4 p$ is involved in metal and heat tolerance. Biometals 16: 369-78

Shen J, Hsu CM, Kang BK, Rosen BP, Bhattacharjee H. 2003b. The Saccharomyces cerevisiae Arr $4 p$ is involved in metal and heat tolerance. Biometals 16: 369-78

Shenton D, Grant CM. 2003. Protein S-thiolation targets glycolysis and protein synthesis in response to oxidative stress in the yeast Saccharomyces cerevisiae. Biochem J 374: 513-9

Shiau AK, Harris SF, Southworth DR, Agard DA. 2006. Structural Analysis of E. coli hsp90 reveals dramatic nucleotide-dependent conformational rearrangements. Cell 127: 329-40

Siegers K, Bolter B, Schwarz JP, Bottcher UM, Guha S, Hartl FU. 2008. TRiC/CCT cooperates with different upstream chaperones in the folding of distinct protein classes. EMBO J 27: 301

Simpson PJ, Schwappach B, Dohlman HG, Isaacson RL. 2010. Structures of Get3, Get4, and Get5 provide new models for TA membrane protein targeting. Structure 18: 897-902

Sinning I, Bange G, Wild K. 2011. It takes two to Get3. Structure 19: 13535

Skrabana R, Cehlar O, Flachbartova Z, Kovac A, Sevcik J, Novak M. 2012. Crystallization and preliminary $\mathrm{X}$-ray diffraction analysis of two peptides from Alzheimer PHF in complex with the MN423 antibody Fab fragment. Acta Crystallogr Sect F Struct Biol Cryst Commun 68: $1186-90$ 
Spiess C, Meyer AS, Reissmann S, Frydman J. 2004. Mechanism of the eukaryotic chaperonin: protein folding in the chamber of secrets. Trends Cell Biol 14: 598-604

Stefanovic S, Hegde RS. 2007. Identification of a targeting factor for posttranslational membrane protein insertion into the ER. Cell 128: 1147-59

Stefer S, Reitz S, Wang F, Wild K, Pang YY, et al. 2011. Structural basis for tail-anchored membrane protein biogenesis by the Get3-receptor complex. Science 333: 758-62

Stengel F, Baldwin AJ, Painter AJ, Jaya N, Basha E, et al. 2010. Quaternary dynamics and plasticity underlie small heat shock protein chaperone function. Proc Natl Acad Sci U S A 107: 2007-12

Storkey C, Davies MJ, Pattison DI. 2014. Reevaluation of the rate constants for the reaction of hypochlorous acid $(\mathrm{HOCl})$ with cysteine, methionine, and peptide derivatives using a new competition kinetic approach. Free Radic Biol Med 73: 60-6

Stromer T, Ehrnsperger M, Gaestel M, Buchner J. 2003. Analysis of the interaction of small heat shock proteins with unfolding proteins. J Biol Chem 278: 18015-21

Sudnitsyna MV, Mymrikov EV, Seit-Nebi AS, Gusev NB. 2012. The role of intrinsically disordered regions in the structure and functioning of small heat shock proteins. Curr Protein Pept Sci 13: 76-85

Suloway CJ, Rome ME, Clemons WM, Jr. 2012. Tail-anchor targeting by a Get3 tetramer: the structure of an archaeal homologue. EMBO J 31 : 707-19

Sun Y, MacRae TH. 2005. Small heat shock proteins: molecular structure and chaperone function. Cell Mol Life Sci 62: 2460-76

Suzuki H, Noguchi S, Arakawa H, Tokida T, Hashimoto M, Satow Y. 2010. Peptide-binding sites as revealed by the crystal structures of the human Hsp40 Hdj1 C-terminal domain in complex with the octapeptide from human Hsp70. Biochemistry 49: 8577-84

Tang G, Peng L, Baldwin PR, Mann DS, Jiang W, et al. 2007. EMAN2: an extensible image processing suite for electron microscopy. J Struct Biol 157: 38-46

Tao YJ, Zheng W. 2011. Chaperones and the maturation of steroid hormone receptor complexes. Oncotarget 2: 104-6

Tapley TL, Franzmann TM, Chakraborty S, Jakob U, Bardwell JC. 2010. Protein refolding by $\mathrm{pH}$-triggered chaperone binding and release. Proc Natl Acad Sci U S A 107: 1071-6

Tapley TL, Korner JL, Barge MT, Hupfeld J, Schauerte JA, et al. 2009. Structural plasticity of an acid-activated chaperone allows promiscuous substrate binding. Proc Natl Acad Sci U S A 106: 555762

Teixeira F, Castro H, Cruz T, Tse E, Koldewey P, et al. 2015. Mitochondrial peroxiredoxin functions as crucial chaperone reservoir in Leishmania infantum. Proc Natl Acad Sci U S A 112: E616-24

Tsutsumishita Y, Onda T, Okada K, Takeda M, Endou H, et al. 1998. Involvement of $\mathrm{H} 2 \mathrm{O} 2$ production in cisplatin-induced nephrotoxicity. Biochem Biophys Res Commun 242: 310-2 
Van Montfort R, Slingsby C, Vierling E. 2001. Structure and function of the small heat shock protein/alpha-crystallin family of molecular chaperones. Adv Protein Chem 59: 105-56

Verghese J, Abrams J, Wang Y, Morano KA. 2012. Biology of the heat shock response and protein chaperones: budding yeast (Saccharomyces cerevisiae) as a model system. Microbiol Mol Biol Rev 76: 115-58

Vijayalakshmi J, Mukhergee MK, Graumann J, Jakob U, Saper MA. 2001. The 2.2 A crystal structure of Hsp33: a heat shock protein with redoxregulated chaperone activity. Structure 9: 367-75

Vilardi F, Stephan M, Clancy A, Janshoff A, Schwappach B. 2014. WRB and CAML are necessary and sufficient to mediate tail-anchored protein targeting to the ER membrane. PLoS One 9: e85033

Voellmy R, Boellmann F. 2007. Chaperone regulation of the heat shock protein response. Adv Exp Med Biol 594: 89-99

Vogel M, Mayer MP, Bukau B. 2006. Allosteric regulation of Hsp70 chaperones involves a conserved interdomain linker. J Biol Chem 281: 38705-11

Voth W, Schick M, Gates S, Li S, Vilardi F, et al. 2014. The protein targeting factor Get3 functions as ATP-independent chaperone under oxidative stress conditions. Mol Cell 56: 116-27

Walter S, Lorimer GH, Schmid FX. 1996. A thermodynamic coupling mechanism for GroEL-mediated unfolding. Proc Natl Acad Sci U S A 93: 9425-30

Wandinger SK, Richter K, Buchner J. 2008. The Hsp90 chaperone machinery. J Biol Chem 283: 18473-7

Wang F, Brown EC, Mak G, Zhuang J, Denic V. 2010. A chaperone cascade sorts proteins for posttranslational membrane insertion into the endoplasmic reticulum. Molecular Cell 40: 159-71

White HE, Orlova EV, Chen S, Wang L, Ignatiou A, et al. 2006. Multiple distinct assemblies reveal conformational flexibility in the small heat shock protein Hsp26. Structure 14: 1197-204

Wilton R, Myatt EA, Stevens FJ. 2004. Analysis of protein-protein interactions by simulation of small-zone gel filtration chromatography. Methods Mol Biol 261: 137-54

Winter J, llbert M, Graf PC, Ozcelik D, Jakob U. 2008. Bleach activates a redox-regulated chaperone by oxidative protein unfolding. Cell 135: 691-701

Winter J, Linke K, Jatzek A, Jakob U. 2005. Severe oxidative stress causes inactivation of DnaK and activation of the redox-regulated chaperone Hsp33. Mol Cell 17: 381-92

Wintrode PL, Friedrich KL, Vierling E, Smith JB, Smith DL. 2003. Solution structure and dynamics of a heat shock protein assembly probed by hydrogen exchange and mass spectrometry. Biochemistry 42: 10667-73

Wong C, Sridhara S, Bardwell JC, Jakob U. 2000. Heating greatly speeds Coomassie blue staining and destaining. Biotechniques 28: 426-8, 30,32

Wood ZA, Poole LB, Karplus PA. 2003a. Peroxiredoxin evolution and the regulation of hydrogen peroxide signaling. Science 300: 650-3 
Wood ZA, Schroder E, Robin Harris J, Poole LB. 2003b. Structure, mechanism and regulation of peroxiredoxins. Trends Biochem Sci 28: $32-40$

Xu Z, Horwich AL, Sigler PB. 1997. The crystal structure of the asymmetric GroEL-GroES-(ADP)7 chaperonin complex. Nature 388: 741-50

Young JC, Agashe VR, Siegers K, Hartl FU. 2004. Pathways of chaperonemediated protein folding in the cytosol. Nat Rev Mol Cell Biol 5: 78191

Young JC, Hoogenraad NJ, Hartl FU. 2003. Molecular chaperones Hsp90 and $\mathrm{Hsp} 70$ deliver preproteins to the mitochondrial import receptor Tom70. Cell 112: 41-50

Zhang HM, McLoughlin SM, Frausto SD, Tang H, Emmett MR, Marshall AG. 2010. Simultaneous reduction and digestion of proteins with disulfide bonds for hydrogen/deuterium exchange monitored by mass spectrometry. Anal Chem 82: 1450-4

Zhou T, Radaev S, Rosen BP, Gatti DL. 2000. Structure of the ArsA ATPase: the catalytic subunit of a heavy metal resistance pump. The EMBO journal 19: 4838-45

Zhu X, Zhao X, Burkholder WF, Gragerov A, Ogata CM, et al. 1996. Structural analysis of substrate binding by the molecular chaperone DnaK. Science 272: 1606-14

Zolkiewski M, Zhang T, Nagy M. 2012. Aggregate reactivation mediated by the Hsp100 chaperones. Arch Biochem Biophys 520: 1-6

Zuehlke A, Johnson JL. 2010. Hsp90 and co-chaperones twist the functions of diverse client proteins. Biopolymers 93: 211-7 


\section{Acknowledgments}

I would like to very sincerely thank Prof. Ursula Jakob who gave me the opportunity to start my scientific career as a Master Student in her lab at the University of Michigan. She saw my potential and encourage me to further proceed with my $\mathrm{PhD}$ in her lab, a decision, I never regret since it gave me the opportunity to grow on a scientific and personal level. Ursula supported me from the first day and was always interested in my work. Furthermore, I would like to express my gratitude to Prof. Blanche Schwappach, who gave me the possibility to have two PhD's for one. She gave me the opportunity to experience the other living side of my project in her lab at the University of Goettingen. Blanche guided me during my stays in her lab and was always supportive of my ideas. Moreover, my special thanks go to Prof. Wolfgang Wintermeyer for supporting my $\mathrm{PhD}$ and for positive input. I also would like to thank Gabriela Brodkorb, who took over the bureaucracy part of my $\mathrm{PhD}$ and helped me to manage my travels between the two labs.

Furthermore, I would like to thank the entire Jakob lab for all the scientific help and all the other non-scientific fun in and outside of the lab. Also, special thanks go to the Bardwell lab, who was always open for scientific input and support. A big thanks goes to the people in the Schwappach lab who welcomed me in their lab and shared great scientific and personal moments with me. Furthermore, a special thank you goes to the students I had the opportunity to supervise during my PhD. Here, I would like to thank Markus Schick, who was a great student and person inside and outside of the lab. I would also like to express my gratitude to all the colleagues who contributed to my project, ahead of all Stephanie Gates who was very supportive with the EM microscopy related part of my thesis and Fabio Vilardi who contributed substantial parts of the in vivo work.

At this point I like to thank all the new friends I made in Ann Arbor and Goettingen for the great time and wonderful experience. Thank you to all my friends who stayed back in Germany, or came over to the US and always supported me from far or close, when I came for a visit, you guys are the 
best. Also, I like to thank a very special person in my life, Marian Perez, who made my years in Ann Arbor so special and unique. You always supported my carrier and decisions, helped me to master my live abroad and became family. Finally, I like to say thank you to my parents Ludmila and Johann Voth and my entire family who supported my travel plans and always where there for me. 


\section{Appendix}

\subsection{Abbreviation}

2D

$3 D$

ADP

ATP

Amp

BSA

${ }^{\circ} \mathrm{C}$

CD

$\mathrm{Cu}^{2+}$

C-terminal

Cys

DNA

DTT

E. coli

EDTA

EM

x g

g two-dimensional

three-dimensional

adenosine diphosphate

adenosine triphosphate

Ampicillin

bovine serum albumin

degree Celsius

circular dichroism

copper(II) ion

carboxyl-terminal

cysteine

deoxyribonucleic acid

dithiothreitol

Escherichia coli

ethylenediaminetetraacetic acid

electron microscope

times gravity

gram 


\begin{tabular}{|c|c|}
\hline $\mathrm{Gdn}-\mathrm{HCl}$ & guanidine hydrochloride \\
\hline h & hour \\
\hline His & histidine \\
\hline Hsp & heat shock protein \\
\hline $\mathrm{H}_{2} \mathrm{O}_{2}$ & hydrogen peroxide \\
\hline $\mathrm{H}_{2} \mathrm{O}$ & water \\
\hline Kan & Kanamycin \\
\hline kDa & kilo Dalton \\
\hline l & liter \\
\hline Leu & leucine \\
\hline $\mathrm{m}$ & milli \\
\hline M & molar \\
\hline MgATP & magnesium ATP \\
\hline $\min$ & minute \\
\hline$\mu$ & micro \\
\hline MS & mass spectroscopy \\
\hline$n$ & nano \\
\hline N-terminal & amino-terminal \\
\hline $\mathrm{NADH}$ & nicotinamide adenine dinucleotide (phosphate) \\
\hline $\mathrm{O}_{2} \cdot^{-}$ & superoxide radicals \\
\hline OD 600 & optical density at $600 \mathrm{~nm}$ \\
\hline
\end{tabular}


$\mathrm{OH} \cdot$

PAR

PCMB

PEG

PMSF

ROS

RT

SBD

SDS

TCA

TCEP

TMD

Trp

Ura

UV

$\mathrm{v} / \mathrm{v}$

$w / v$ hydroxyl radicals

4-(2-pyridylazo) resorcinol

para-chloromercurybenzoic acid

polyethylene glycol

phenylmethane sulfonyl fluoride

reactive oxygen species

room temperature

substrate binding domain

sodium dodecyl sulfate

Trichloroacetic acid

tris(2-carboxyethyl)phosphine

transmembrane domain

tryptophan

uracil

ultraviolet

volume per volume

weight per volume 


\subsection{Index of figures}

Figure 1. 1: A complex chaperone network maintains the proteostasis in the cell.............. 4

Figure 1. 2: The Hsp70 chaperone cycle ............................................................ 7

Figure 1. 3: Mechanism of Hsp33's chaperone activation .............................................. 16

Figure 1. 4: Hsp33's redox-mediated chaperone function .......................................... 18

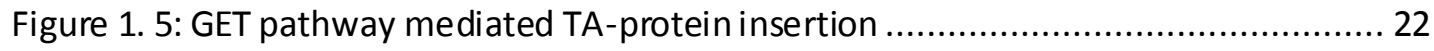

Figure 1. 6: Get3 is a highly conserved P-loop ATPase............................................... 23

Figure 1. 7 Structure of S. cerevisiae Get3 dimer.......................................................... 25

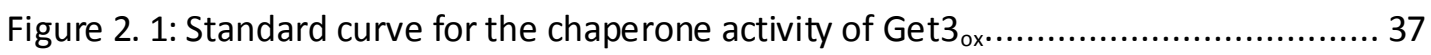

Figure 2. 2: Formation and purification of the Get $3_{\text {tet }}$-Luciferase complex...................... 44

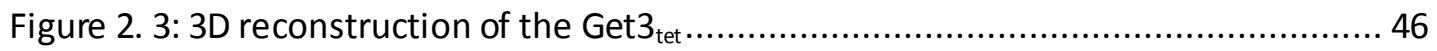

Figure 3.1. 1: Get3's conserved cysteines are essential for yeast cell survival under oxidative

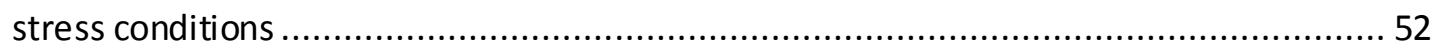

Figure 3.1. 2: Get3 exhibits chaperone activity when oxidized....................................... 53

Figure 3.1. 3: ROS-mediated activation of Get3's chaperone function ............................. 54

Figure 3.1. 4: Copper activates Get3's chaperone function through oxidation ................... 56

Figure 3.1. 5: Chaperone activity of oxidized Get3 is ATP-independent .......................... 57

Figure 3.1. 6: Get3's chaperone function is activated through thiol oxidation................... 58

Figure 3.1. 7: Oxidation mediated disulfide bond formation in Get3 ..............................6 60

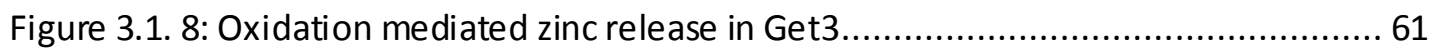

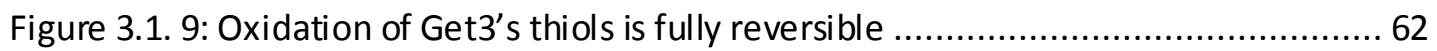

Figure 3.1. 10: Activation of Get3's chaperone function is a reversible process ................6 64

Figure 3.1. 11: Oxidized Get3 undergoes reversible structural rearrangements .................65 65

Figure 3.1. 12: Oxidation reversibly alters Get3's protein characteristics........................66 66

Figure 3.1. 13: Fully inactivated Get $3_{\text {red }}$ shows no chaperone activity ............................6 67

Figure 3.1. 14: Identification of smal lest chaperone-active Get $3_{\text {ox }}$ unit.......................... 68

Figure 3.1. 15: The smallest chaperone-active Get $3_{\text {ox }}$ unit is a tetramer..........................69

Figure 3.1. 16: Get3 oligomers imaged on negative-stain EM grids ................................ 70

Figure 3.1. 17: 3D modeling of the Get3 ${ }_{\text {ox }}$ tetramer based on negative-stain EM data....... 72

Figure 3.1. 18: Coverage map for pepsin digested Get3 ........................................... 74

Figure 3.1. 19: Differences in deuterium incorporation between Get3 ${ }_{\text {red }}$ and Get $3_{\text {ox }} \ldots \ldots . . . .75$

Figure 3.1. 20: Get3 undergoes massive conformational rearrangements upon oxidation. 76

Figure 3.1. 21: Conformational rearrangements mapped on Get3's structure .................. 78

Figure 3.1. 22: Get3's chaperone activity is independent of its TA protein targeting function

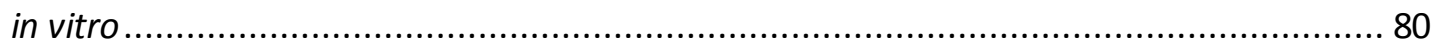

Figure 3.1. 23: Get3 rescues yeast cells under oxidative stress condition independent of its

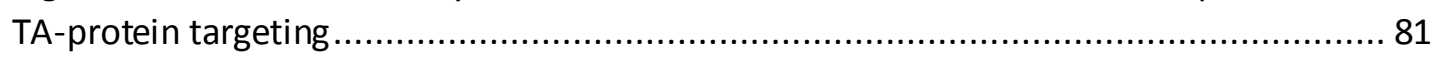

Figure 3.1. 24: Get3 I193D is deficient in TA-protein targeting in vivo ............................ 83

Figure 3.1. 25: Get3 accumulates at deposition sites of mistargeted Sed5 under stress conditions.

Figure 3.2. 1: Reduction of chaperone active Get $3_{\text {ox }}$ in complex with substrate is not

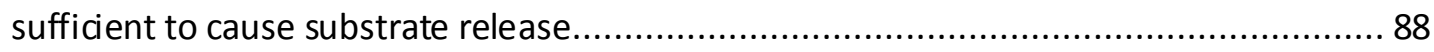

Figure 3.2. 2: Substrate binding and transfer ability of Get3 is redox-dependent.............. 89

Figure 3.2. 3: Get $3_{0 x}$ maintains luciferase in a refolding competent state ......................... 91 
Figure 3.2. 4: Get3's substrate transfer to eukaryotic Hsp40/70 is redox dependent........ 93 Figure 3.2. 5: The oxidized $\mathrm{Get} 3_{\text {tet }}$ forms a stable complex with luciferase....................... 95

Figure 3.2. 6: Purification of a homogeneous Get $3_{\text {tet }}$-luciferase complex......................... 96

Figure 3.2. 7: The Get $3_{\text {tet }}$-luciferase complex forms oligomers of various sizes ................ 97

Figure 3.2. 8: Get $_{\text {tet }^{-}}$-luciferase complex formation is detectible on negative stain EM ..... 99

Figure 4. 1: Get3's dual function in yeast cells ......................................................103

Figure 4. 2: Redox-regulated Get3 is part of the proteostasis network..........................111 


\subsection{Publications}

Voth W, Schick M, Gates S, Li S, Vilardi F, Gostimskaya I, Southworth DR, Schwappach B, Jakob U. The protein targeting factor Get3 functions as ATP-independent chaperone under oxidative stress conditions. Mol Cell 2014;56:116-27.

Voth W, Gates S, Schafer-Nielsen C, Farkas À, Vergara FJC, Kilisch M, Southworth DR, Schwappach B, Jakob U. The ATP-independent chaperone Get3 protects soluble protein under oxidative stress condition in yeast (Manuscripts in preparation)

\subsection{Meetings and Conferences}

Midwest Stress Response and Molecular Chaperone Meeting (January 2016), Northwestern University, Evanston, USA

The Protein Targeting Factor Get3 Functions as ATP-Independent Chaperone under Oxidative

Voth W, Schick M, Gates S, Li S, Vilardi F, Gostimskaya I, Southworth DR, Schwappach B, Jakob U. (poster presentation)

ESF-EMBO Thiol-based Redox switches in Life Sciences (September 2015), Sant Feliu de Guixols, Spain

The Protein Targeting Factor Get3 Functions as ATP-Independent Chaperone under Oxidative Stress

Voth W, Schick M, Gates S, Li S, Vilardi F, Gostimskaya I, Southworth DR, Schwappach B, Jakob U. (poster presentation)

2nd Symposium of the DFG Priority Program SPP1710 (September 2014), Heidelberg, Germany

Get3 Functions as ATP-Independent Chaperone Under Oxidative Stress Conditions (oral presentation)

Voth W, Schick M, Gates S, Li S, Vilardi F, Gostimskaya I, Southworth DR, Schwappach B, Jakob U. 